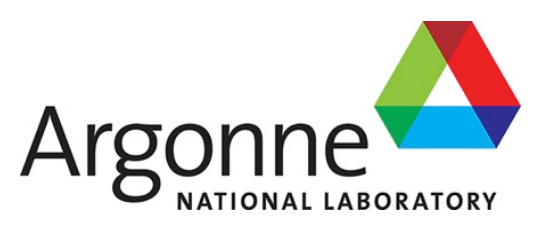

ANL/NSE-19/18

\title{
SAM User's Guide
}

Nuclear Science \& Engineering Division 
About Argonne National Laboratory

Argonne is a U.S. Department of Energy laboratory managed by UChicago Argonne, LLC

under contract DE-AC02-06CH11357. The Laboratory's main facility is outside Chicago,

at 9700 South Cass Avenue, Argonne, Illinois 60439. For information about Argonne

and its pioneering science and technology programs, see www.anl.gov.

\section{DOCUMENT AVAILABILITY}

Online Access: U.S. Department of Energy (DOE) reports produced after 1991 and a growing number of pre-1991 documents are available free at OSTI.GOV (http://www.osti.gov/), a service of the U.S. Dept. of Energy's Office of Scientific and Technical Information

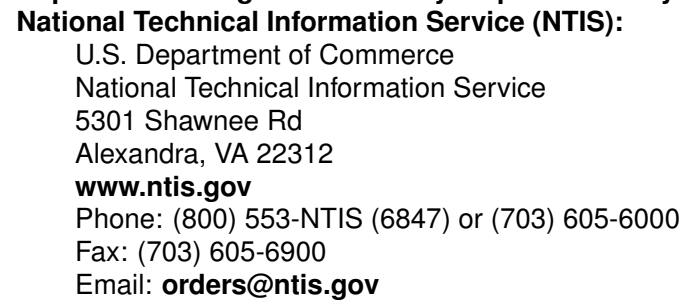

Reports not in digital format are available to DOE and DOE contractors from the Office of Scientific and Technical Information (OSTI):

U.S. Department of Energy

Office of Scientific and Technical Information

P.O. Box 62

Oak Ridge, TN 37831-0062

www.osti.gov

Phone: (865) 576-8401

Fax: (865) 576-5728

Email: reports@osti.gov

\section{Disclaimer}

This report was prepared as an account of work sponsored by an agency of the United States Government. Neither the United States Government nor any agency thereof, nor UChicago Argonne, LLC, nor any of their employees or officers, makes any warranty, express or implied, or assumes any legal liability or responsibility for the accuracy, completeness, or usefulness of any information, apparatus, product, or process disclosed, or represents that its use would not infringe privately owned rights. Reference herein to any specific commercial product, process, or service by trade name, trademark, manufacturer, or otherwise, does not necessarily constitute or imply its endorsement, recommendation, or favoring by the United States Government or any agency thereof. The views and opinions of document authors expressed herein do not necessarily state or reflect those of the United States Government or any agency thereof, Argonne National Laboratory, or UChicago Argonne, LLC. 


\section{SAM User's Guide}

prepared by

Rui Hu, Ling Zou, Guojun Hu

Nuclear Science \& Engineering Division, Argonne National Laboratory

August 2019 


\begin{abstract}
The System Analysis Module (SAM) is a modern system analysis tool being developed at Argonne National Laboratory for advanced non-LWR safety analysis. It aims to provide fast-running, whole-plant transient analyses capability with improved-fidelity for Sodium-cooled Fast Reactors (SFR), Lead-cooled Fast Reactors (LFR), and Molten Salt Reactors (MSR) or Fluoride-cooled High-temperature Reactors (FHR). SAM takes advantage of advances in physical modeling, numerical methods, and software engineering to enhance its user experience and usability. It utilizes an object-oriented application framework (MOOSE), and its underlying meshing and finite-element library (libMesh) and linear and non-linear solvers (PETSc), to leverage the modern advanced software environments and numerical methods.

This document provides a user's guide, which will help users understand the input description and core capabilities of the SAM code. A brief overview of the code is presented, as well as how to obtain and run it. The input syntax for various parts of the code is provided. Additionally, a number of example problems, starting with simple unit component problems to problems with increasing complexity, are provided. Because the code is still under active development, this SAM User's Guide will evolve with periodic updates.
\end{abstract}




\section{Contents}

$\begin{array}{ll}\text { ABSTRACT } & \text { ii }\end{array}$

Contents $\quad$ vi

List of Figures $\quad$ vii

List of Tables $\quad$ viii

1 SAM Overview 1

1.1 Ultimate Goals and Objectives . . . . . . . . . . . . . . . . . . 1

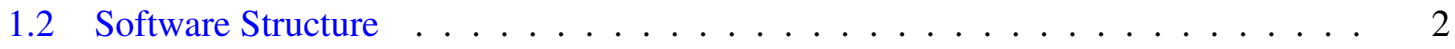

1.3 Governing Theory . . . . . . . . . . . . . . . . . . . 2

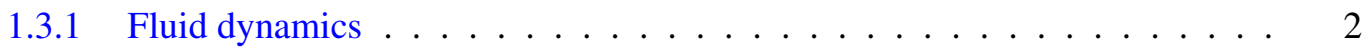

1.3.2 Heat transfer . . . . . . . . . . . . . . . . . . 3

1.3 .3 Closure models . . . . . . . . . . . . . . . . . 3

1.3.4 Mass transport model development . . . . . . . . . . . . . . . 3

1.3.5 Reactor kinetics model development . . . . . . . . . . . . . . . . 4

1.3.6 Numerical method . . . . . . . . . . . . . . . . . . . 4

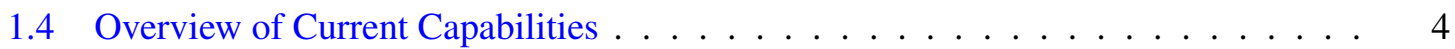

2 Running SAM 6

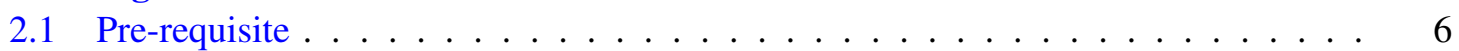

2.2 Obtaining the Code . . . . . . . . . . . . . . . . . . . . . . . 6

2.3 Compiling the Code from Source . . . . . . . . . . . . . . . . 7

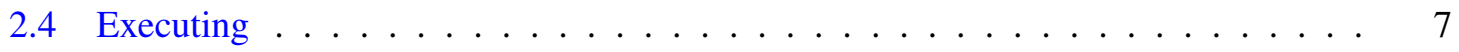

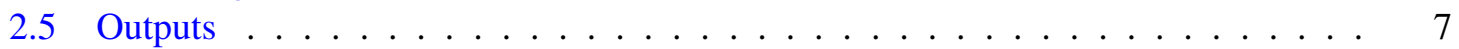

3 SAM Components $\quad 8$

4 Input File Syntax $\quad 12$

4.1 Global Parameters . . . . . . . . . . . . . . . . . . . . . . . . 12

4.2 Equation of State $($ EOS $) \quad \ldots \ldots \ldots \ldots \ldots \ldots \ldots$

$4.2 .1 \quad$ Built-in EOS . . . . . . . . . . . . . . . . . . 17

4.2 .2 Simple Linearized EOS . . . . . . . . . . . . . . . . . . . . . . . . . . . . . . 17

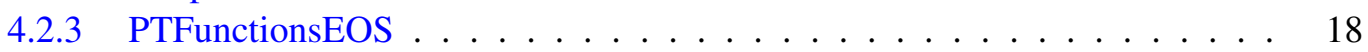

4.2 .4 PTFluidPropertiesEOS . . . . . . . . . . . . . . . . . . . . . . . . . . 19

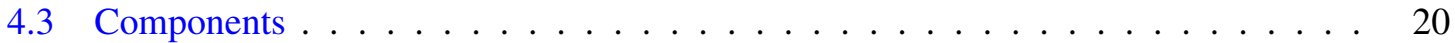

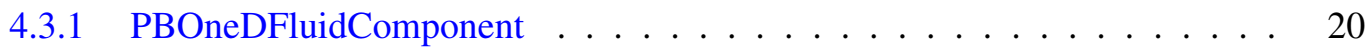

4.3 .2 Heatstructure . . . . . . . . . . . . . . . . . . 30

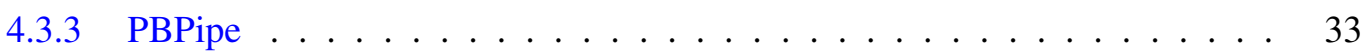

4.3.4 PBCoreChannel . . . . . . . . . . . . . . . . . . . . 36

4.3.5 PBDuctedCoreChannel . . . . . . . . . . . . . . . . . 41

4.3.6 PBBypassChannel . . . . . . . . . . . . . . . . . . . . 42

4.3.7 PBMoltenSaltChannel . . . . . . . . . . . . . . . . . . . . 43 
4.3 .8 FuelAssembly . . . . . . . . . . . . . . . . . . . . . . 44

4.3 .9 DuctedFuelAssembly . . . . . . . . . . . . . . . . . . . . . . . 47

4.3.10 MultiChannelRodBundle . . . . . . . . . . . . . . . . . . . 48

4.3.11 HexLatticeCore . . . . . . . . . . . . . . . . . . . . 51

4.3.12 PBCoupledHeatStructure . . . . . . . . . . . . . . 53

4.3.13 HeatStructureWithExternalFlow . . . . . . . . . . . . . . 56

4.3.14 HeatTransferWithExternalHeatStructure . . . . . . . . . . . . . . 59

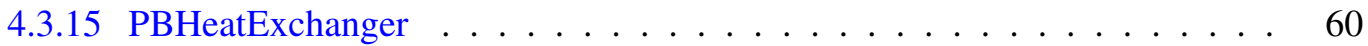

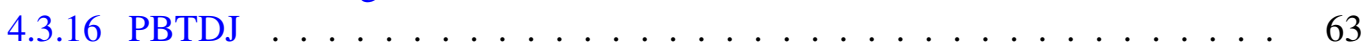

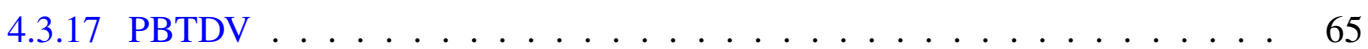

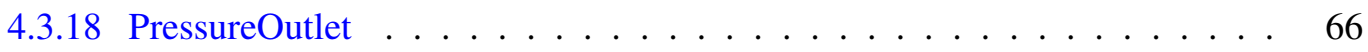

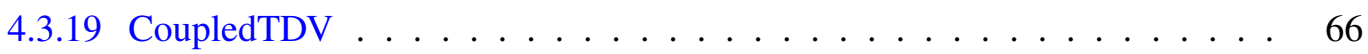

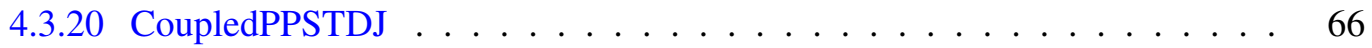

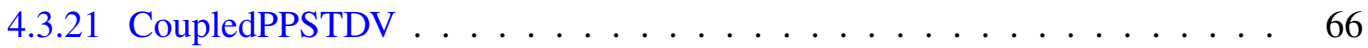

4.3 .22 PBSingleJunction . . . . . . . . . . . . . . . . 67

4.3 .23 PBBranch . . . . . . . . . . . . . . . . . . . 67

4.3 .24 PBVolumeBranch . . . . . . . . . . . . . . . . . . . . . . . 69

4.3.25 PBLiquidVolume . . . . . . . . . . . . . . . . . . . 70

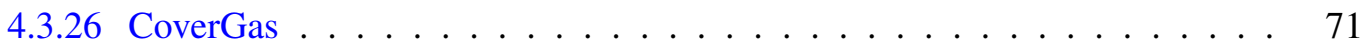

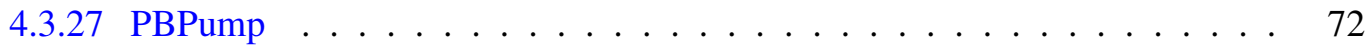

4.3.28 StagnantVolume . . . . . . . . . . . . . . . . . 73

4.3 .29 LiquidTank . . . . . . . . . . . . . . . . . . . . . . . . . . . . . . . . . . . . . . . . . . 74

4.3 .30 ReactorCore . . . . . . . . . . . . . . . . . . . 75

4.3.31 SurfaceCoupling . . . . . . . . . . . . . . . . . 76

4.3.32 ReactorPower . . . . . . . . . . . . . . . . . . . . . . 77

4.3.33 PointKinetics . . . . . . . . . . . . . . . . . . 78

4.3.34 ReferenceBoundary . . . . . . . . . . . . . . . . 82

4.3 .35 PipeChain . . . . . . . . . . . . . . . . . . . . . . 82

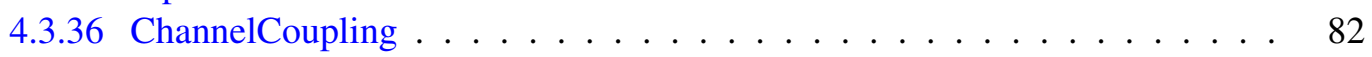

4.3.37 HeatPipe and HeatPipeArray . . . . . . . . . . . . . . . . . 83

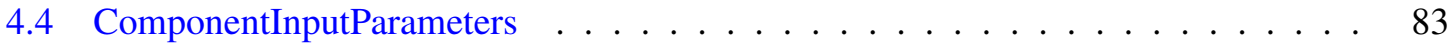

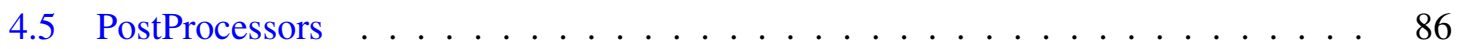

4.5.1 ComponentBoundaryEnergyBalance . . . . . . . . . . . . 86

4.5.2 ComponentBoundaryFlow . . . . . . . . . . . . . . . . . 87

4.5.3 ComponentBoundaryScalarFlow . . . . . . . . . . . . . . 87

4.5.4 ComponentBoundary VariableValue . . . . . . . . . . . . . 88

4.5.5 ComponentNodalVariableValue . . . . . . . . . . . . . . 88

4.5.6 ConductionHeatRemovalRate . . . . . . . . . . . . . 88

4.5.7 HeatExchangerHeatRemovalRate . . . . . . . . . . . . . . . 89

4.6 TimeSteppers . . . . . . . . . . . . . . . . . . . . . . . . . . . . . . . . . . . 89

4.6.1 CourantNumberTimeStepper . . . . . . . . . . . . . . . 89

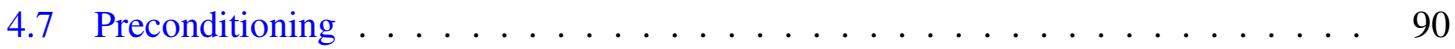

4.8 Executioner . . . . . . . . . . . . . . . . . . . . . . . . . . 92

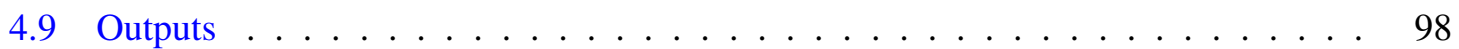


5 Example Problems $\quad 108$

5.1 Heat Conduction Problem . . . . . . . . . . . . . . . . . . . . . 108

5.2 Single Channel Flow . . . . . . . . . . . . . . . . . . . . . . . 113

5.3 Core Channel . . . . . . . . . . . . . . . . . . . . . . . 116

5.4 Heat Exchanger . . . . . . . . . . . . . . . . . . . . 120

5.5 Volume Branch . . . . . . . . . . . . . . . . . . . . . . . 124

5.6 A Simple Loop Model . . . . . . . . . . . . . . . . . . . . . . . 130

5.7 A Simplified SFR Model . . . . . . . . . . . . . . . . . . . . 135

ACKNOWLEDGMENTS 154

$\begin{array}{ll}\text { REFERENCES } & 154\end{array}$ 


\section{List of Figures}

1.1 SAM Code Structure . . . . . . . . . . . . . . . . . . . . . 3

1.2 SAM simulation results of an SFR . . . . . . . . . . . . . . . 5

4.1 SAM PBOneDFluidComponent examples. . . . . . . . . . . . . . . . 22

4.2 PBOneDFluidComponent with end element refinements. . . . . . . . . . . . 23

4.3 Fuel bundles in (a) square-lattice, typically seen in light water reactor designs; and (b) hexagonal-lattice, typically seen in sodium fast reactor designs. . . . . . . . . . 25

4.4 Typical SFR wire-wrapped rod configuration. . . . . . . . . . . . . . . . 26

4.5 An example of two-dimensional plate type of heat structure. . . . . . . . . . . 32

4.6 SAM's PBPipe component, which consists of a PBOneDFluidComponent to model the one-dimensional fluid flow and one layer (or several layers) of HeatStructure to model its wall. . . . . . . . . . . . . . . . . .

4.7 An input example of PBPipe with two layers of heat structures to model its wall. For example, it could represent a layer of metal wall and an extra layer of thermal insulation material. . . . . . . . . . . . . . . . . . . .

4.8 PBCoreChannel component (a) SAM's PBCoreChannel component simulates the average coolant flow in rod bundles and heat conduction inside a fuel rod; and (b) An example mesh used in the PBCoreChannel component, 2-D mesh for heat structure and 1-D mesh for fluid flow. . . . . . . . . . . . . . . 37

4.9 Sketch of the regions in the multi-channel model . . . . . . . . . . . . . . . . 49

4.10 Sketch of HexLatticeCore component. . . . . . . . . . . . . . . . 52

4.11 Two types of PBHeatExchanger component designs. As an example, the two figures show shell-and-tube heat exchanger design. . . . . . . . . . . . .

4.12 The PBLiquidVolume concept used in SAM. (a) PBLiquidVolume with ambient pressure as its reference pressure; (b) PBLiquidVolume with an external CoverGas to specify its reference pressure. . . . . . . . . . . . . . 70

5.1 SAM model of the 2-D heat conduction problem. . . . . . . . . . . . . . 108

5.2 Comparisons of centerline temperature distributions of the heated rod, 2D conduction. 110

5.3 Example of SAM results shown in Paraview. . . . . . . . . . . . . . . . . 113

5.4 Transient responses of the pipe under inlet temperature oscillation, BDF2 . . . . . . 114

5.5 The schematic of the spatial discretization of the core channel problem. . . . . . 116

5.6 Temperature distribution of a counter-current heat exchanger. . . . . . . . . . . 120

5.7 The three-pipe-in and two-pipe-out VolumeBranch test model. . . . . . . . . . . . 124

5.8 Input parameters of the three pipe in and two pipe out VolumeBranch test model. . 125

5.9 Transient temperature response at the VolumeBranch and pipe outlets. . . . . . . 125

5.10 Schematics of the a test loop problem. . . . . . . . . . . . . . 130

5.11 Schematics of the a simple pool-type SFR model. . . . . . . . . . . . . . . 135

\section{List of Tables}

2.1 Software Libraries Used by SAM . . . . . . . . . . . . . . . . . . . 6

3.1 List of boundary condition type of components of SAM . . . . . . . . . 8

3.2 List of junction type of components of SAM . . . . . . . . . . . . . . . 9

3.3 List of non-geometric type of components of SAM $\ldots \ldots \ldots$ 
3.4 List of geometric and assembly type of components of SAM . . . . . . . . . 11

4.1 SAM components that supports ComponentInputParameters feature . . . . . . . . 84

5.1 Comparison of SAM and Analytical Solutions for the Steady State Axial-Radial Heat Conduction Problem . . . . . . . . . . . . . . . . . . . . . . . . . . . . 109 


\section{SAM Overview}

The System Analysis Module (SAM) [1, 2, 3, 4] is an advanced system analysis tool being developed at Argonne National Laboratory under the U.S. Department of Energy (DOE) Nuclear Energy Advanced Modeling and Simulation (NEAMS) program. It aims to be a modern system analysis code, which takes advantage of the advancements software design, numerical methods, and physical models over the past two decades. SAM focuses on modeling advanced reactor concepts such as SFRs (sodium fast reactors), LFRs (lead-cooled fast reactors), and FHRs (fluoride-saltcooled high temperature reactors) or MSRs (molten salt reactors). These advanced concepts are distinguished from light-water reactors (LWR) in their use of single-phase, low-pressure, hightemperature, and low Prandtl number (sodium and lead) coolants. This simple yet fundamental change has significant impacts on core and plant design, the types of materials used, component design and operation, fuel behavior, and the significance of the fundamental physics in play during transient plant simulations.

SAM is aimed to solve the tightly-coupled physical phenomena including heat generation, heat transfer, fluid dynamics, and thermal-mechanical response in reactor structures, systems and components in a fully-coupled fashion but with reduced-order modeling approaches to facilitate rapid turn-around for design and safety optimization studies. As a new code development, the initial effort focused on developing modeling and simulation capabilities of the heat transfer and single-phase fluid dynamics, as well as reactor point kinetics responses in reactor systems. This Chapter discusses goals and objectives, software structure, the governing theory, as well as current capabilities of the code. In the coming years, the SAM code will continuously mature as a modern system analysis tool for advanced (non-LWR) reactor design optimization, safety analyses, and licensing support.

\subsection{Ultimate Goals and Objectives}

The ultimate goal of SAM is to be used in advanced reactor safety analysis for design optimization and licensing support. The important physical phenomena and processes that may occur in reactor systems, structures, and components shall be of interest during reactor transients including Anticipated Operational Occurrence (AOO), Design Basis Accident (DBA), and additional postulated accidents but not including severe accidents. Typical reactor transients include: loss of coolant accidents, loss of flow events, excessive heat transfer events, loss of heat transfer events, reactivity and core power distribution events, increase in reactor coolant inventory events, and anticipated transients without scram (ATWS).

As a modern system analysis code, SAM is also envisioned to expand beyond the traditional system analysis code to enable multi-dimensional flow analysis, containment analysis, and source term analysis, either through reduced-order modeling in SAM or via coupling with other simulation tools. Additionally, the regulatory processes in the United States is being evolved to a risk-informed approach that is based on first understanding the best-estimate behavior of the fuel, the reactor, the reactor coolant system, the engineered safeguards, the balance of plant, operator actions, and all of the possible interactions among these elements. To enable this paradigm, an advanced system analysis code such as SAM must be able to model the integrated response of all of these physical systems and considerations to obtain a best-estimate simulation that includes both validation and uncertainty quantification. 
The SAM code is aimed to provide improved-fidelity simulations of transients or accidents in an advanced non-LWR, including three-dimension resolutions as needed or desired. This will encompass the fuel rod, the fuel assembly, the reactor, the primary and intermediate heat transport system, the balance-of-plant, the containment. Multi-dimension, multi-scale, and multi-physics effects will be captured via coupling with other simulation tools, and computational accuracy and efficiency will be state-of-the-art. Uncertainty quantification will be integrated into SAM numerical simulations. Legacy issues such as numerical diffusion and stability in traditional system codes will be addressed and the code will attract broad use across the nuclear energy community based on its performance and many advantages relative to the legacy codes. The integrated architecture will provide a robust toolset for decision making with full consideration of the various disciplines and technologies affecting an issue.

\subsection{Software Structure}

SAM is being developed as a system-level modeling and simulation tool with higher fidelity (compared to existing system analysis tools), and with well-defined and validated simulation capabilities for advanced reactor systems. It provides fast-running, modest-fidelity, whole-plant transient analyses capabilities. To fulfill the code development, SAM utilizes the object-oriented application framework MOOSE [5] and its underlying meshing and finite-element library libMesh [6] and linear and non-linear solvers PETSc [7], to leverage the available advanced software environments and numerical methods. The high-order spatial discretization schemes, fully implicit and high-order time integration schemes, and the advanced solution method (such as the Jacobian-free Newton-Krylov (JFNK) method [8]) are the key aspects in developing an accurate and computationally efficient model in SAM.

The software structure of SAM is illustrated in Figure 1.1. In addition to the fundamental physics modeling of the single-phase fluid flow and heat transfer, SAM incorporates advances in the closure models (such as convective heat transfer correlations) for reactor system analysis developed over the past several decades. A set of Components, which integrate the associated physics modeling in the component, have been developed for friendly user interactions. This component-based modeling strategy is similar to what is implemented in RELAP-7 [9], which is also a MOOSE-based system analysis tool (focused on LWR simulations). A flexible coupling interface has been developed in SAM so that multi-scale, multi-physics modeling capabilities can be achieved by integrating with other higher-fidelity or conventional simulation tools.

\subsection{Governing Theory}

\subsubsection{Fluid dynamics}

Fluid dynamics is the main physical model of the SAM code. SAM employs a standard onedimensional transient model for single-phase incompressible but thermally expandable flow. The governing equations consist of the continuity equation, momentum equation, and energy equations. A three-dimensional module is also under development to model the multi-dimensional flow and thermal stratification in the upper plenum or the cold pool of an SFR. Additionally, a subchannel module will be developed for fuel assembly modeling. 


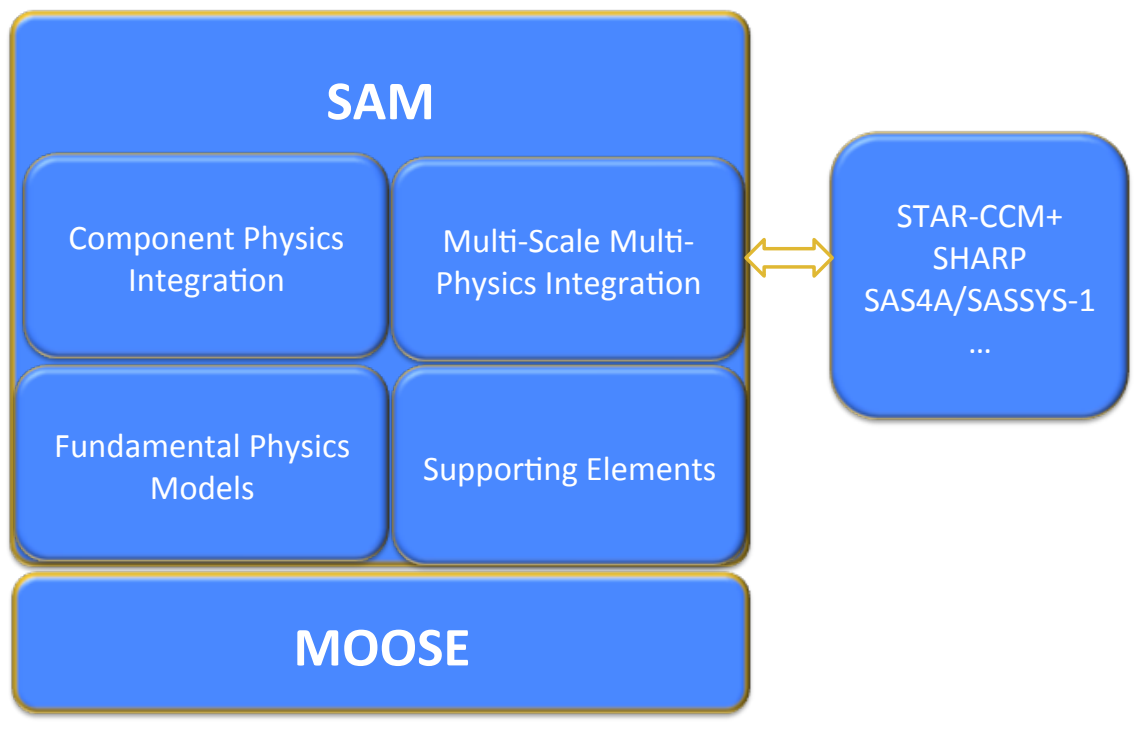

Figure 1.1: SAM Code Structure

\subsubsection{Heat transfer}

Heat structures model heat conduction inside solids and permit the modeling of heat transfer at interfaces between solid and fluid components. Heat structures are represented by one-dimensional or two-dimensional heat conduction in Cartesian or cylindrical coordinates. Temperature-dependent thermal conductivities and volumetric heat capacities can be provided in tabular or functional form. Heat structures can be used to simulate the temperature distributions in solid components such as fuel pins or plates, heat exchanger tubes, and pipe and vessel walls, as well as to calculate the heat flux conditions for fluid components. Flexible conjugate heat transfer and thermal radiation modeling capabilities are also implemented in SAM.

\subsubsection{Closure models}

The fluid equation of state (EOS) model is required to complete the governing flow equations, which are based on the primitive variable formulation; therefore, the dependency of fluid properties and their partial derivatives on the state variables (pressure and temperature) are implemented in the EOS model. Some fluid properties, such as sodium, air, salts like FLiBe and FLiNaK, have been implemented in SAM. Empirical correlations for friction factor and convective heat transfer coefficient are also required in SAM because of its one-dimension approximation of the flow field. The friction and heat transfer coefficients are dependent on flow geometries as well as operating conditions during the transient.

\subsubsection{Mass transport model development}

The mass transport modeling capability is needed to model sources and transport of particles for a number of applications, such as tritium transport, delayed neutron precursor drift, radioactive isotope transport for molten salt fueled/cooled systems. A general passive scalar transport model 
has been implemented in SAM, and it can be used to track any number of species carried by the fluid flow.

\subsubsection{Reactor kinetics model development}

SAM employs a built-in point kinetics model, including reactivity feedback and decay heat modeling. Various reactivity feedback mechanisms are included, such as the axial and radial expansion feedbacks due to thermal expansion and displacement effects. The effects of delay neutron precursor drift in MSRs can also be modeled.

\subsubsection{Numerical method}

SAM is a finite-element-method based code. The "weak forms" of the governing equations are implemented in SAM. It uses the Jacobian-Free Newton Krylov (JFNK) solution method to solve the equation system. The JFNK method uses a multi-level approach, with outer Newton's iterations (nonlinear solver) and inner Krylov subspace methods (linear solver), in solving large nonlinear systems. The concept of 'Jacobian-free' is proposed, because deriving and assembling large Jacobian matrices could be difficult and expensive. The JFNK method has become an increasingly popular option for solving large nonlinear equation systems and multi-physics problems, as observed in a number of different disciplines [8]. One feature of JFNK is that all the unknowns are solved simultaneously in a fully coupled fashion. This solution scheme avoids the errors from operator splitting and is especially suitable for conjugate heat transfer problems in which heat conduction in a solid is tightly coupled with fluid flow.

\subsection{Overview of Current Capabilities}

To develop a system analysis code, numerical methods, mesh management, equations of state, fluid properties, solid material properties, neutronics properties, pressure loss and heat transfer closure laws, and good user input/output interfaces are all indispensable. SAM leverages the MOOSE framework and its dependent libraries to provide JFNK solver schemes, mesh management, and I/O interfaces while focusing on new physics and component model development for advanced reactor systems. The developed physics and component models provide several major modeling features:

1. One-D pipe networks represent general fluid systems such as the reactor coolant loops.

2. Flexible integration of fluid and solid components, able to model complex and generic engineering system. A general liquid flow and solid structure interface model was developed for easier implementation of physics models in the components.

3. A pseudo three-dimensional capability by physically coupling the 1-D or 2-D components in a 3-D layout. For example, the 3-D full-core heat-transfer in an SFR reactor core can be modeled. The heat generated in the fuel rod of one fuel assembly can be transferred to the coolant in the core channel, the duct wall, the inter-assembly gap, and then the adjacent fuel assemblies.

4. Pool-type reactor specific features such as liquid volume level tracking, cover gas dynamics, heat transfer between 0-D pools, fluid heat conduction, etc. These are important features for accurate safety analyses of SFRs or other advanced reactor concepts. 
5. A computationally efficient multi-dimensional flow model is under development, mainly for thermal mixing and stratification phenomena in large enclosures for safety analysis. It was noted that an advanced and efficient thermal mixing and stratification modeling capability embedded in a system analysis code is very desirable to improve the accuracy of advanced reactor safety analyses and to reduce modeling uncertainties.

6. A general mass transport capability has been implemented in SAM based on the passive scalar transport. The code can track any number of species carried by the fluid flow for various applications.

7. An infrastructure for coupling with external codes has been developed and demonstrated. The code coupling with STAR-CCM+ [10], SAS4A/SASSYS-1 [11], Nek5000, and BISON [12] have been demonstrated, while the coupling with PRONGHORN, RattleSnake, and PORTEUS codes are ongoing or being planned.

An example of SAM simulation results of an SFR is shown in Figure 1.2.

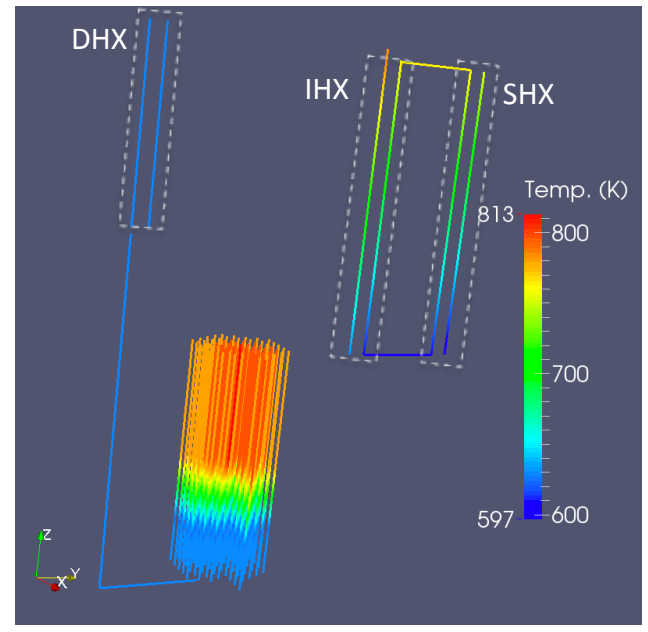

(a) SAM model with 61 core channels

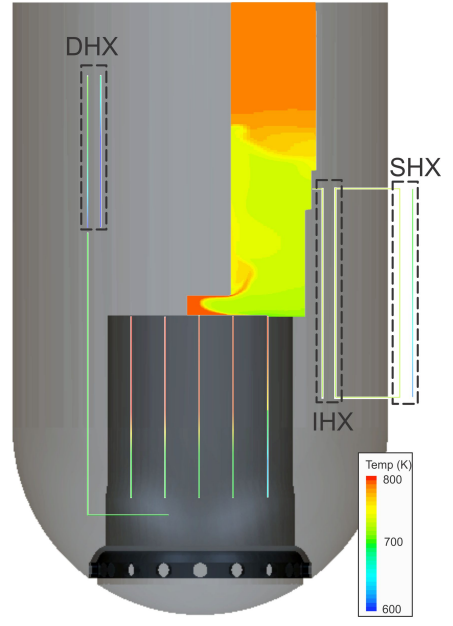

(b) Coupled SAM and CFD code simulation

Figure 1.2: SAM simulation results of an SFR. 


\section{Running SAM}

\subsection{Pre-requisite}

SAM is built on the computational framework MOOSE (Multi-physics Object-Oriented Simulation Environment) to interface with LibMesh and PETSc to provide the underlying geometry (mesh I/O) and numerical capabilities (finite element library and solvers). It requires all of the code dependencies as MOOSE requires. A summary of the dependent libraries of SAM is listed in Table 2.1.

Table 2.1: Software Libraries Used by SAM

\begin{tabular}{|l|l|l|}
\hline Library & Origin & Purpose \\
\hline MOOSE [5] & Idaho National Laboratory & $\begin{array}{l}\text { Computational framework, interfaces } \\
\text { other libraries }\end{array}$ \\
\hline LibMesh [6] & University of Texas, Austin & Finite element library \\
\hline PETSc [7] & Argonne National Laboratory & Parallel linear and nonlinear solvers \\
\hline Hypre (optional) [13] & Argonne National Laboratory & Parallel linear and nonlinear solvers \\
\hline MPICH [14] & Argonne National Laboratory & Message passing/parallel processing \\
\hline TBB (optional) [15] & Intel Corporation & Multi-thread parallelism \\
\hline
\end{tabular}

The MOOSE development team maintains a compiled set of all dependencies, except MOOSE and LibMesh, on the public MOOSE website (http://mooseframework.org/) with precompiled packages containing Petsc, Hypre, MPICH, and TBB for several Mac OS and Linux systems. MOOSE and LibMesh are available from the MOOSE GitHub site (https: //gi thub . com/idaholab/ moose.git). For advanced users, all the dependent libraries are open- source codes and can thus be downloaded and compiled on Mac OS and Linux systems. The instructions for installing the MOOSE dependency package, and for compiling Libmesh and MOOSE can also be found at the public MOOSE website, http://www. mooseframework.org/.

\subsection{Obtaining the Code}

SAM is hosted in a private, access-controlled Git repository at Argonne National Laboratory. All changes to the source code are committed with revision number and comments, and are tracked in the repository. Contact the author of this User's Guide if interested in obtaining the code.

After obtaining access to the code, one could use git commands to obtain the source code of SAM:

\section{git clone <repo_site_address>}

MOOSE is set as a submodule of SAM, so that a reliable version of MOOSE is always available and consistent with the product version of SAM. The MOOSE submodule can be obtained by:

git submodule update -init

or by 


\subsection{Compiling the Code from Source}

After obtained the MOOSE submodule, one would need to compile libMesh first before compiling MOOSE and SAM. Under the moose/scripts directory, the libMesh can be obtained and compiled by:

$$
\text { ./update_and_rebuild_libmesh.sh }
$$

After that, MOOSE and SAM can be compiled by use the default Makefile from the repository under the SAM folder. Use

$$
\text { make }
$$

to compile the code on a single processor; or use

$$
\text { make }-j<n>
$$

to compile the code on $n$ processors.

\subsection{Executing}

SAM, due to its dependence on MOOSE, is not compatible with Windows operating systems. However it is fully compatible with Linux, Unix, and MacOS. It can be run from the shell prompt.

The execution command looks like:

sam-opt -i <input_file_name $>$

Many example test problems can be found under /tests/ subdirectory.

\subsection{Outputs}

SAM supports all MOOSE output file formats. It typically writes solution data to an ExodusII file, and write post-processor and scalar variables to a separate comma separated values (CSV) file. Several options exist for viewing ExodusII output files. One good choice is to use the open-source software Paraview (www. paraview.org). The CSV file uses table-structured format, which can be opened by many software such as Microsoft Excel. 


\section{SAM Components}

The physics modeling (fluid flow and heat transfer) and mesh generation of individual reactor components are encapsulated as Component classes in SAM along with some component specific models. A set of components has been developed based on the finite-element fluid model and heat conduction model, including:

1. basic geometric components;

2. 0-D components for setting boundary conditions;

3. 0-D components for connecting 1-D components;

4. assembly components by combining the basic geometric components and the 0-D connecting components; and

5. non-geometric components for physics integration.

A brief description of major SAM components is listed in Tables 3.1 - 3.4. The physics models associated with these components will be discussed in SAM Theory Manual, and the input format is discussed in Section 4.

Table 3.1: List of boundary condition type of components of SAM

\begin{tabular}{|l|l|l|}
\hline Component name & Descriptions & Dimension \\
\hline PBTDJ & $\begin{array}{l}\text { An inlet boundary in which the flow velocity and } \\
\text { temperature are provided by pre-defined functions. }\end{array}$ & 0 -D \\
\hline CoupledPPSTDJ & $\begin{array}{l}\text { CoupledPPSTDJ is a special PBTDJ component that } \\
\text { is designed to facilitate MultiApp simulations. }\end{array}$ & 0 -D \\
\hline PBTDV & $\begin{array}{l}\text { A boundary in which pressure and temperature con- } \\
\text { ditions are provided by pre-defined functions. }\end{array}$ & 0 -D \\
\hline CoupledTDV & $\begin{array}{l}\text { A time-dependent-volume boundary in which } \\
\text { boundary conditions are provided by other codes in } \\
\text { coupled code simulation. }\end{array}$ & 0 -D \\
\hline CoupledPPSTDV & $\begin{array}{l}\text { CoupledPPSTDV is a special PBTDV component } \\
\text { that is designed to facilitate MultiApp simulations. }\end{array}$ & $0-\mathrm{D}$ \\
\hline PressureOutlet & A subset of PBTDV, will be removed. & $0-\mathrm{D}$ \\
\hline ReferenceBoundary & $\begin{array}{l}\text { ReferenceBoundary component provides a fixed } \\
\text { value boundary condition to a one-dimensional fluid } \\
\text { type of component. }\end{array}$ & $0-\mathrm{D}$ \\
\hline StagnantVolume & $\begin{array}{l}\text { Models a stagnant liquid volume, with connections } \\
\text { to other 0-D volumes but no connections to 1-D fluid } \\
\text { components. }\end{array}$ & $0-\mathrm{D}$ \\
\hline
\end{tabular}


Table 3.2: List of junction type of components of SAM

\begin{tabular}{|l|l|l|}
\hline Component name & Descriptions & Dimension \\
\hline PBSingleJunction & $\begin{array}{l}\text { Models a zero-volume flow joint, where only two 1- } \\
\text { D fluid components are connected. }\end{array}$ & 0-D \\
\hline PBBranch & $\begin{array}{l}\text { Models a zero-volume flow joint, where multiple 1- } \\
\text { D fluid components are connected. }\end{array}$ & 0-D \\
\hline PBVolumeBranch & $\begin{array}{l}\text { Considering the volume effects of a PBBranch com- } \\
\text { ponent so that it can account for the mass and energy } \\
\text { in-balance between inlets and outlets due to inertia. }\end{array}$ & 0-D \\
\hline PBPump & $\begin{array}{l}\text { Simulates a pump component, in which the pump } \\
\text { head is dependent on a pre-defined function. }\end{array}$ & 0-D \\
\hline PBLiquidVolume & $\begin{array}{l}\text { A 0-D liquid volume with cover gas (the liquid level } \\
\text { is tracked and the volume can change during the } \\
\text { transient). }\end{array}$ & 0-D \\
\hline LiquidTank & $\begin{array}{l}\text { The LiquidTank component of SAM simulates a PB- } \\
\text { VolumeBranch (or PBLiquidVolume) and the heat } \\
\text { structure (modeled as PBCoupledHeatStructure) at- } \\
\text { tached to it in order to capture this additional thermal } \\
\text { inertia. }\end{array}$ & or 2-D structure \\
\hline
\end{tabular}


Table 3.3: List of non-geometric type of components of SAM

\begin{tabular}{|l|l|l|}
\hline Component name & Descriptions & Dimension \\
\hline ReactorCore & $\begin{array}{l}\text { Models a pseudo three-dimensional reactor core; It } \\
\text { consists of member core channels (with duct walls) } \\
\text { and bypass channels. }\end{array}$ & $\begin{array}{l}\text { 1-D fluid, 1-D } \\
\text { or 2-D structure }\end{array}$ \\
\hline CoverGas & $\begin{array}{l}\text { A 0-D gas volume that is connected to one or multi- } \\
\text { ple liquid volumes. }\end{array}$ & 0-D \\
\hline SurfaceCoupling & $\begin{array}{l}\text { The SurfaceCoupling component models the heat } \\
\text { transfer between two solid surfaces, suitable for ra- } \\
\text { diation heat transfer or gap heat transfer between } \\
\text { them. }\end{array}$ & ND \\
\hline ChannelCoupling & $\begin{array}{l}\text { A non-geometric component for coupling two 1-D } \\
\text { fluid ND components (with energy exchange). }\end{array}$ & ND \\
\hline ReactorPower & $\begin{array}{l}\text { A non-geometric component describing the total re- } \\
\text { actor ND power. }\end{array}$ & ND \\
\hline PointKinetics & $\begin{array}{l}\text { The PointKinetics component is the build-in point } \\
\text { kinetics model of SAM, which models the transient } \\
\text { behaviors of reactor fission power, delayed-neutron } \\
\text { precursors, as well as reactivity feedback from other } \\
\text { components, e.g., core channels. }\end{array}$ & \\
\hline PipeChain & $\begin{array}{l}\text { A non-geometric component for connecting a num- } \\
\text { ber of ND fluid components. }\end{array}$ & ND \\
\hline $\begin{array}{l}\text { HeatTransferWith } \\
\text { ExternalHeatStructure }\end{array}$ & $\begin{array}{l}\text { A non-geometric component for connecting a num- } \\
\text { ber of ND fluid components. }\end{array}$ & ND \\
\hline
\end{tabular}


Table 3.4: List of geometric and assembly type of components of SAM

\begin{tabular}{|c|c|c|}
\hline Component name & Descriptions & Dimension \\
\hline PBOneDFluidComponent & $\begin{array}{l}\text { Simulates 1-D fluid flow using the primitive variable } \\
\text { based fluid model }\end{array}$ & $1-\mathrm{D}$ \\
\hline HeatStructure & $\begin{array}{l}\text { Simulates 1-D or 2-D heat conduction inside solid } \\
\text { structures }\end{array}$ & 1-D or $2-\mathrm{D}$ \\
\hline PBCoupledHeatStructure & $\begin{array}{l}\text { The heat structure connecting two liquid compo- } \\
\text { nents (1-D or } 0-D) \text {. }\end{array}$ & 1-D or $2-\mathrm{D}$ \\
\hline PBPipe & $\begin{array}{l}\text { Simulates fluid flow in a pipe and heat conduction in } \\
\text { the pipe wall. }\end{array}$ & $\begin{array}{l}\text { 1-D fluid, 1-D } \\
\text { or 2-D structure }\end{array}$ \\
\hline PBHeatExchanger & $\begin{array}{l}\text { Simulates a heat exchanger, including the fluid flow } \\
\text { in the primary and secondary sides, convective heat } \\
\text { transfer, and heat conduction in the tube wall. }\end{array}$ & $\begin{array}{l}\text { 1-D fluid, 1-D } \\
\text { or 2-D structure }\end{array}$ \\
\hline PBCoreChannel & $\begin{array}{l}\text { Simulates reactor core channels, including 1-D flow } \\
\text { channel and the inner heat structures (fuel, gap, and } \\
\text { clad) of the fuel rod. }\end{array}$ & $\begin{array}{l}\text { 1-D fluid, 1-D } \\
\text { or 2-D structure }\end{array}$ \\
\hline PBDuctedCoreChannel & $\begin{array}{l}\text { Simulates reactor core channels with an outer heat } \\
\text { structure of the duct wall. }\end{array}$ & $\begin{array}{l}\text { 1-D fluid, 1-D } \\
\text { or 2-D structure }\end{array}$ \\
\hline PBBypassChannel & $\begin{array}{l}\text { Models the bypass flow in the gaps between fuel as- } \\
\text { semblies. }\end{array}$ & $1-\mathrm{D}$ \\
\hline FuelAssembly & $\begin{array}{l}\text { Models reactor fuel assemblies composed of multi- } \\
\text { ple CoreChannels, representing different regions of } \\
\text { a fuel assembly (core, gas plenum, reflector, shield, } \\
\text { etc.). }\end{array}$ & $\begin{array}{l}\text { 1-D fluid, 1-D } \\
\text { or 2-D structure }\end{array}$ \\
\hline DuctedFuelAssembly & $\begin{array}{l}\text { Model reactor fuel assemblies composed of multiple } \\
\text { DuctedCoreChannels. }\end{array}$ & $\begin{array}{l}\text { 1-D fluid, 1-D } \\
\text { or 2-D structure }\end{array}$ \\
\hline MultiChannelRodBundle & $\begin{array}{l}\text { Models the rod bundle with a multi-channel model, } \\
\text { in which multiple CoreChannels and the inter- } \\
\text { channel mixing are defined and created. }\end{array}$ & $\begin{array}{l}\text { 1-D fluid, 1-D } \\
\text { or 2-D structure }\end{array}$ \\
\hline HexLatticeCore & $\begin{array}{l}\text { Models a hexagonal lattice core, in which the } \\
\text { CoreChannels and HeatStructures are defined and } \\
\text { created. }\end{array}$ & $\begin{array}{l}\text { 1-D fluid, 1-D } \\
\text { or 2-D structure }\end{array}$ \\
\hline PBMoltenSaltChannel & $\begin{array}{l}\text { PBMoltenSaltChannel is a component intended to } \\
\text { model the core behavior of molten-salt reactor de- } \\
\text { signs. }\end{array}$ & $1-\mathrm{D}$ \\
\hline HeatPipe & HeatPipe is a component to model heat pipes. & $\begin{array}{l}\text { 1-D fluid, 1-D } \\
\text { or 2-D structure }\end{array}$ \\
\hline HeatPipeArray & $\begin{array}{l}\text { HeatPipeArray models an array of HeatPipe compo- } \\
\text { nents. }\end{array}$ & $\begin{array}{l}\text { 1-D fluid, 1-D } \\
\text { or 2-D structure }\end{array}$ \\
\hline $\begin{array}{l}\text { HeatStructure } \\
\text { WithExternalFlow }\end{array}$ & $\begin{array}{l}\text { HeatStructureWithExternalFlow is also a } \\
\text { HeatStructure-based component similar to PB- } \\
\text { CoupledHeatStructure, however with the main } \\
\text { purpose to facilitate code-to-code coupling via its } \\
\text { boundary surfaces. }\end{array}$ & $\begin{array}{l}1-\mathrm{D} \text { or } 2-\mathrm{D} \\
\text { structure }\end{array}$ \\
\hline
\end{tabular}




\section{Input File Syntax}

SAM uses a block-structured input file. Each block is identified with square brackets. The opening brackets contain the type of the input block and the empty brackets mark the end of the block. Each block may contain sub-blocks. Each sub-block must have a unique name when compared with all other sub-blocks in the current block.

Line inputs are given as parameter and value pairs with an equal sign between them. They specify parameters to be used by the object being described. The parameter is a string, and the value may be a string, a Boolean value, an integer, a real number, or a list of strings, integers, or real numbers. Lists are given in single quotes and are separated by whitespace. Sub-blocks normally contain a type line input. This line specifies the particular type of object being described.

All units used in SAM are SI units. This standardizes the model input by eliminating the possibility of errors caused by using one set of units for one model and another set of units for a different model. "\#” symbol indicates comments in the input file and can be located anywhere in the input file.

A quick example is given to demonstrate the basic block-structured syntax of SAM input file:

\begin{tabular}{|c|c|c|}
\hline & \# Beginning of an input block \\
\hline \multicolumn{2}{|l|}{$\begin{array}{l}\text { [BlockName ] } \\
\text { RealNumber }\end{array}$} & \# This specifies a real number \\
\hline \multirow{2}{*}{$\begin{array}{l}\text { Boolean } \\
\text { MyString }\end{array}$} & $=$ true & \# This specifies a boolean value \\
\hline & $=S A M$ & $\begin{array}{l}\text { \# This specifies a string value } \\
\text { \# An empty line will be simply ignored }\end{array}$ \\
\hline [./ SubBlockName] & & \# Beginning of an input sub-block \\
\hline Numbers & $=11.0 \quad 2.0 \quad 3.0^{\prime}$ & \# This specifies a list of numbers \\
\hline Strings & $=$ 'Hello World' & \# This specifies a list of strings \\
\hline$[\ldots /]$ & & \# Ending of an input sub-block \\
\hline [] & & \# Ending of an input block \\
\hline
\end{tabular}

The following subsections have brief descriptions of each block used in SAM input. This User's Guide is intended to help users understand the basics of the SAM code and learn how to run it. The details of the input parameters and modeling options will be discussed in a more detailed User's Manual in the future when the SAM code becomes more mature.

\subsection{Global Parameters}

The GlobalParams block specifies the global parameters used by the code such as global initial conditions, the scaling factors for the primary variable residuals, etc. The modeling parameters associated with the primitive-variable-based fluid model can be defined in the PBModelParams sub-block.

The full list of input parameters of the GlobalParams block is shown below. The line inputs are listed in a three-column format, with the first column showing the available input parameters, the second column showing the default value of the input parameters, and the third column showing a short description of the input parameters. "= (required)" is listed in some cases in the second column for parameter in other input blocks, which indicates that the parameter must be provided in the input file otherwise the code cannot be executed.

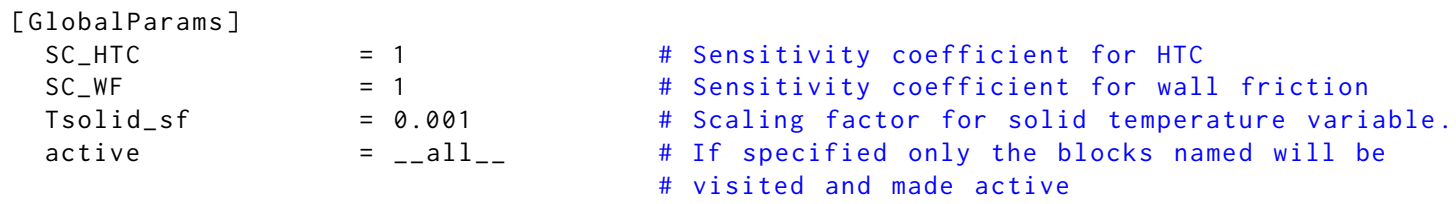




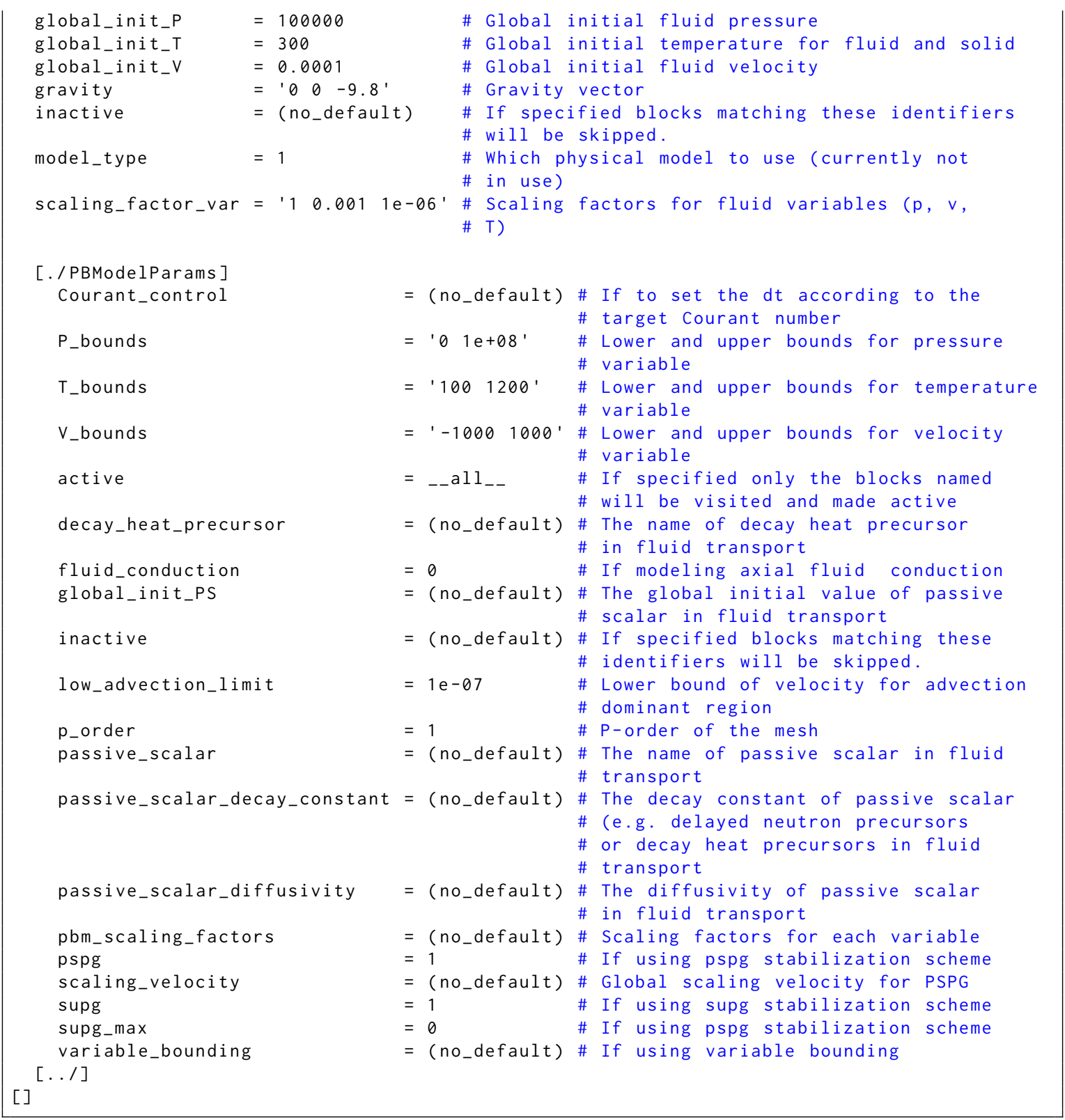

For each input parameter in the GlobalParams input block, details are provided as follows:

- global_init_P

As the name suggests, it specifies the global initial value of fluid pressure, which however can be overridden by initial values specified locally in the component level, for example, initial_P of PBOneDFluidComponent (section 4.3.1).

If not specified, a default value, $10^{5} \mathrm{~Pa}$, is used for this parameter.

- global_init_V

This input parameter specifies the global initial value of fluid velocity, which can also be overrid- 
den by initial values specified locally in the component level, for example, initial_V of PBOneDFluidComponent (section 4.3.1).

If not specified, a default value, $10^{-4} \mathrm{~m} / \mathrm{s}$, is used.

- global_init_T

This input parameter specifies the global initial value of fluid temperature, which can also be overridden by initial values specified locally in the component level, for example, initial_T of PBOneDFluidComponent (section 4.3.1).

If not specified, a default value, $300 \mathrm{~K}$, is used.

- scaling_factor_var

This input parameter specifies the scaling factors to the residuals of three fluid equations, i.e., mass, momentum, and energy equations. The default values are ' $10.0011 \mathrm{e}-06$ ', which general work pretty well for most cases.

- Tsolid_sf

This input parameter specifies the scaling factor to the residual of heat conduction equation in solids, e.g., heat structures. The default value is 0.001 .

- SC_HTC

This input parameter works as a global multiplier to the heat transfer coefficient used in the code, e.g., $\mathrm{HTC}_{\text {new }}=\mathrm{HTC}_{\text {original }} \times \mathrm{SC}$ _HTC. Similar to those global initial values, this parameter can also be overridden locally in the component level, for example, SC_HTC of PBOneDFluidComponent (section 4.3.1).

- SC_WF

This input parameter works as a global multiplier to the wall friction coefficient used in the code, e.g., $f_{\text {new }}=\mathrm{f}_{\text {original }} \times \mathrm{SC}_{-}$WF. This parameter can also be overridden locally in the component level, for example, SC_WF of PBOneDFluidComponent (section 4.3.1).

- gravity

This input parameter is the vector form of gravitational constant in $(\mathrm{x}, \mathrm{y}, \mathrm{z})$ coordinates. The default value is ' 0 c 0 - 9.8 '.

- model_type

This input parameter specifies the model type used in SAM simulation, 1 for one-dimensional model (default value), and 2 for three-dimensional model. However, currently this input parameter is not used.

Input parameters of the input sub-block, PBModelParams, is discussed as follows:

- Courant_control

This is a boolean type of input parameter, by default, false. If specified true, the code uses the maximum Courant number (automatically calculated) as an indicator to control the time step size during a transient simulation. It is used in combination of the CourantNumberTimestepper PostProcessor, see section 4.6.1. 
- variable_bounding

This input parameter specifies if variables bounding should be applied to the main fluid variables, i.e., pressure, velocity, and temperature. By default, it is false, i.e., no bounding is applied.

- P_bounds, T_bounds, and V_bounds

These input parameters specify the bounds for the three main fluid variables. The default values are: P_bounds $=' 01.0 \mathrm{e} 8{ }^{\prime} \mathrm{Pa}, \mathrm{V}_{-}$bounds $='-1.0 \mathrm{e}-31.0 \mathrm{e} 3{ }^{\prime} \mathrm{m} / \mathrm{s}$, and T_bounds $=' 1001.2 \mathrm{e} 3^{\prime}$ $\mathrm{K}$. These bounds are only applied when variable_bounding $=$ true.

- fluid_conduction

This input parameter specifies if axial heat conduction effect of the fluid should be modeled, which, if modeled, would be included in the fluid energy equation. Such an effect is generally only important in applications where high thermal-conductivity fluids, such as liquid metals, are used. An example application is sodium-cooled fast reactor analysis. For most other applications, it is safe to not include this effect.

- passive_scalar

This input parameter accepts a list of names of passive scalars that are passively transported with fluid flow. For example, passive_scalar = 'particle1 particle2 particle3'.

- global_init_PS

This input parameter specifies the global initial values of passive scalars. For example, global_init_PS $=' 10.080 .020 .0^{\prime}$. Similar to fluid properties, such as pressure, this global initial condition could be overridden by locally specified initial conditions in in the component level, for example, initial_PS of PBOneDFluidComponent (section 4.3.1).

- passive_scalar_diffusivity

This input parameter specifies the diffusivities of passive scalars in fluid.

- passive_scalar_decay_constant

This input parameter specifies the decay constants of passive scalars. If part of the passive_scalar list is also defined as decay_heat_precursor, the corresponding decay constants will be used as decay heat precursor decay constant to compute decay power.

- decay_heat_precursor

This input parameter defines a list of decay heat precursors, each of which must have been specified in the passive_scalar list, to compute decay power.

- p_order

This input parameter specifies the p-order of one- and two-dimensional meshes generated within the code. The default value is 1 , i.e., first-order.

- pbm_scaling_factors

This input parameter works similarly to the higher level global input parameter, scaling_factor_var. If specified, it overrides scaling_factor_var. 
- pspg

This input parameter specifies if PSPG stabilization should be used in the fluid mass equation. By default, it is true (1).

- scaling_velocity

This input parameter specifies a reference velocity for scaling to be used in the PSPG scheme. Currently, not used.

- supg

This input parameter specifies if SUPG stabilization should be used in the fluid momentum and energy equations. By default, it is true (1).

- supg_max

In some extreme cases, for example, fluid velocities very close to 0 . The FEM scheme may not be stable enough to cause unphysical oscillations in numerical solutions. With supg_max = true, stabilization parameters are adjusted to larger values that help suppress such non-physical oscillations. In most cases, this is not needed, and it is false (0), by default.

- low_advection_limit

This parameter specifies the lower bound of velocity for advection dominant region. When the velocity magnitude is smaller than this value, SUPG stabilization scheme is deemed to be unnecessary, and is turned off. The default value of this input parameter is $10^{-7} \mathrm{~m} / \mathrm{s}$.

An example input of the GlobalParams block is shown below. Note that only a small fraction of the parameters were provides. For other unprovided input parameters, default values are used if they are available in the code (as listed in the above input description). If the default value is not available, the parameter is not required and its intended function is not activated.

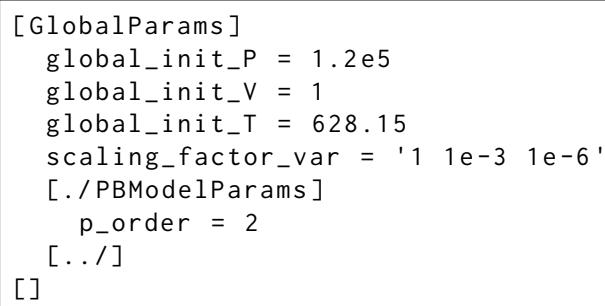

Another example is given on passive scalars. There are eight passive scalars specified in PBModelParams, six of which are also defined as decay_heat_precursor.

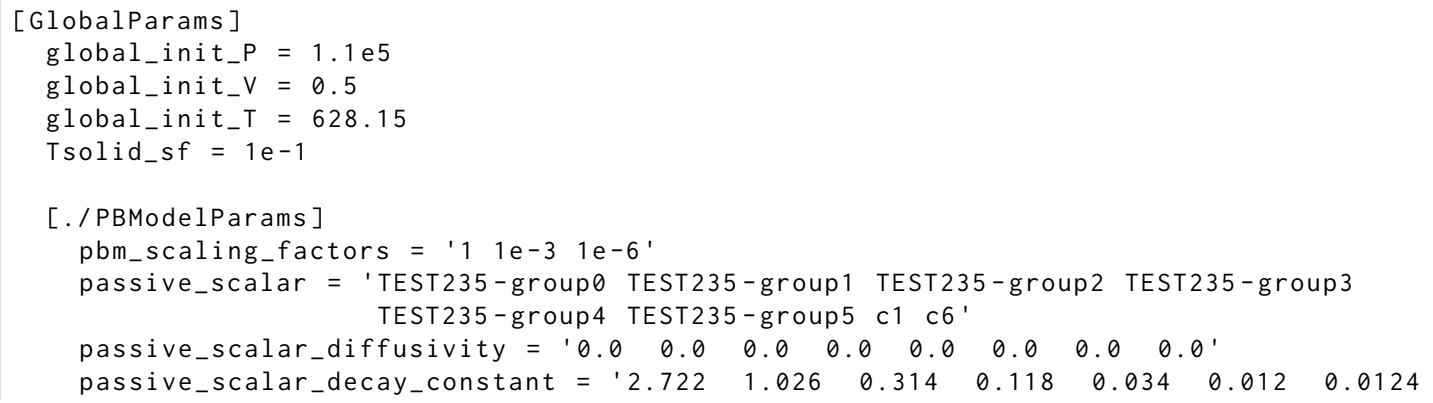




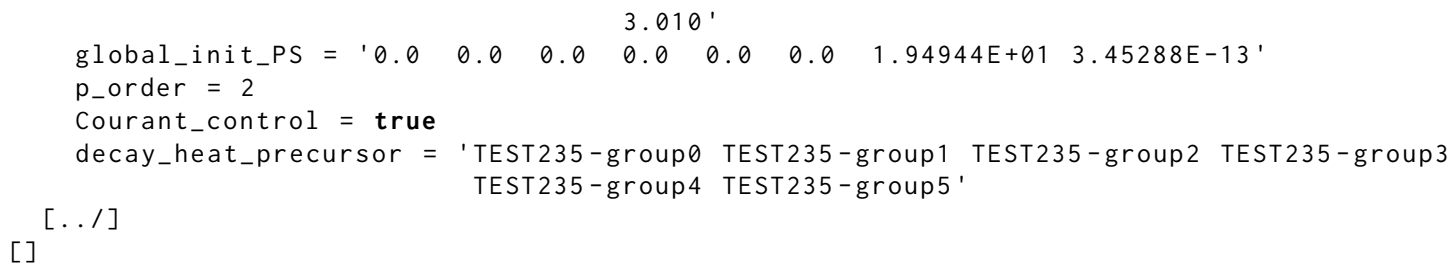

\subsection{Equation of State (EOS)}

SAM provides different options in specifying fluid properties in simulations. Users could choose from SAM's built-in fluid library for commonly-used fluids, including air, nitrogen, helium, sodium, two types of molten salt (Flibe and Flinak), and one simulant oil (DowthermA).

\subsubsection{Built-in EOS}

SAM provides several built-in EOS for users to pick from. These model requires minimum input effort, and examples are given as follows:

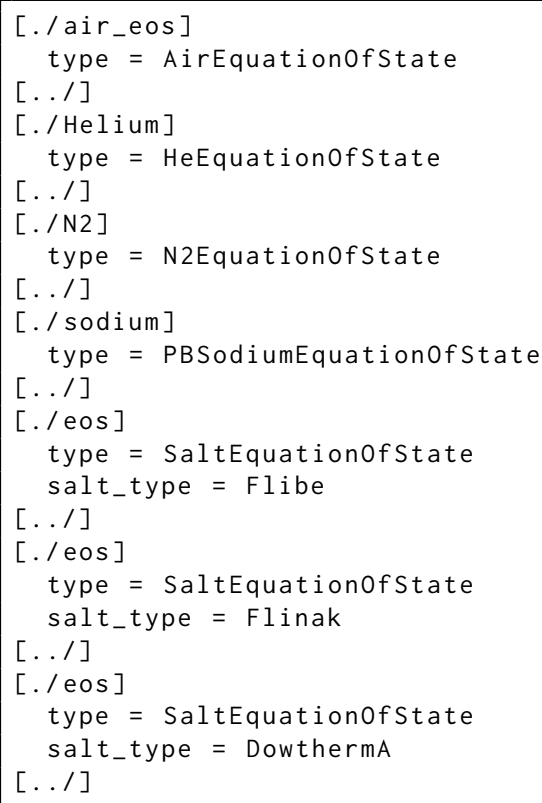

\subsubsection{Simple Linearized EOS}

SAM also provides another simple equation of state, in which all properties, except density and specific enthalpy, are constant user-specified input values. The complete input parameters of this simple equation of state is given as follows:

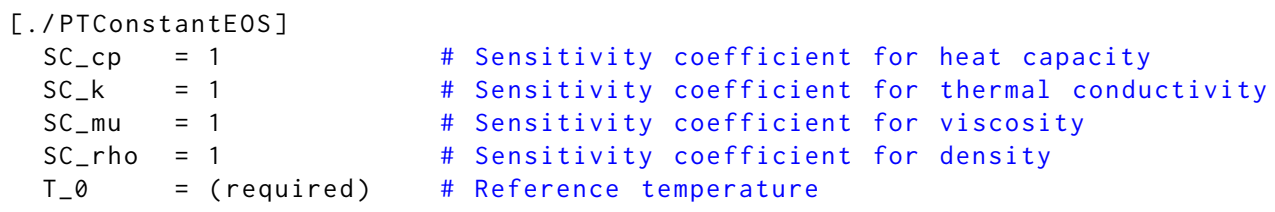




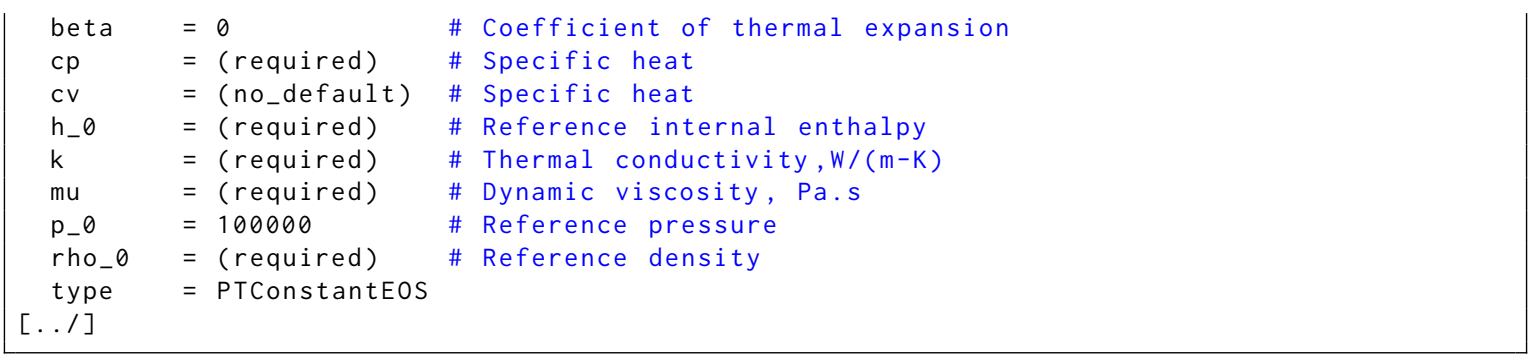

Density is a linear function of temperature using the provided thermal expansion coefficient, $\beta$, which is calculated as:

$$
\rho=\rho_{0}-\rho_{0} \beta\left(T-T_{0}\right)
$$

Specific enthalpy is also linearly dependent on temperature,

$$
h=h_{0}+c_{p}\left(T-T_{0}\right)
$$

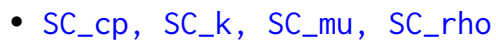

These are sensitivity coefficients that are multiplied to the values of specific heat, thermal conductivity, viscosity, and density of the fluid. They are most useful for uncertainty quantification, and by default, are zero. For normal applications, they could be simply ignored. These parameters are available for all equation of states implemented in SAM code, including those built-in fluid library discussed earlier.

- $\mathrm{p}_{-} 0$

A reference pressure with default value of $10^{5} \mathrm{~Pa}$. For this EOS, it is not used and safe to leave it unspecified.

Other input parameters are self-explanatory and thus not discussed further. An example is given as follows:

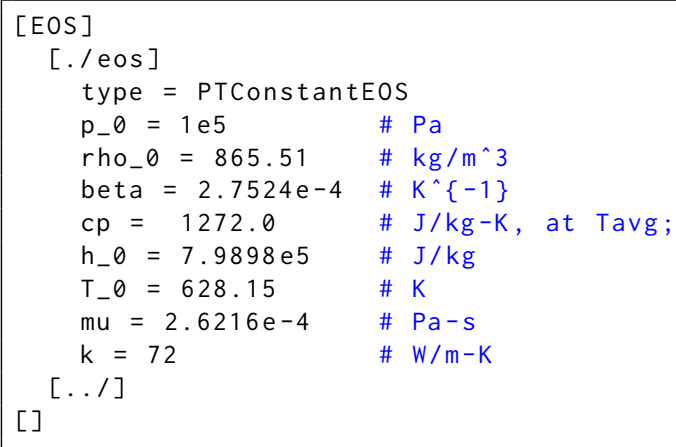

\subsubsection{PTFunctionsEOS}

In addition to the simple linearized equation of state, SAM also provides PTFunctionsEOS to accept more complex user-defined fluid properties in terms of pressure and temperature-dependent functions. Its input parameters are listed as follows: 


\begin{tabular}{|c|c|c|}
\hline \\
\hline \multicolumn{3}{|r|}{ \# Sensitivity coefficient for heat capacity } \\
\hline SC_k & $=1$ & \# Sensitivity coefficient for thermal conductivity \\
\hline SC_mu & $=1$ & \# Sensitivity coefficient for viscosity \\
\hline SC_rho & $=1$ & \# Sensitivity coefficient for density \\
\hline beta & $=($ required $)$ & \# Coefficient of thermal expansion \\
\hline$c p$ & $=($ required $)$ & \# Specific heat \\
\hline enthalpy & $=($ required $)$ & \# enthalpy \\
\hline k & $=($ required $)$ & \# Thermal conductivity, $W /(m-K)$ \\
\hline $\mathrm{mu}$ & $=($ required $)$ & \# Dynamic viscosity, Pa.s \\
\hline$p_{-} \theta$ & $=100000$ & \# Reference pressure \\
\hline rho & $=($ required) & \# Density \\
\hline type & $=$ PTFunctionsEOS & \\
\hline$[\ldots /]$ & & \\
\hline
\end{tabular}

Among these input parameters, $\mathrm{SC}_{-} \mathrm{cp}, \mathrm{SC}_{-} \mathrm{k}, \mathrm{SC}_{-} \mathrm{mu}, \mathrm{SC}_{-} r h o$, and $\mathrm{p}_{-} 0$, are the same as described in section 4.2.2. Other parameters are described as follows:

- rho, beta, cp, mu, k, enthalpy (required)

All these input parameters are required. Each of them accepts either a constant value or a function name, which should have been specified in the [Functions] input block.

An example of using 'PTFunctionsEOS' is given as follows:

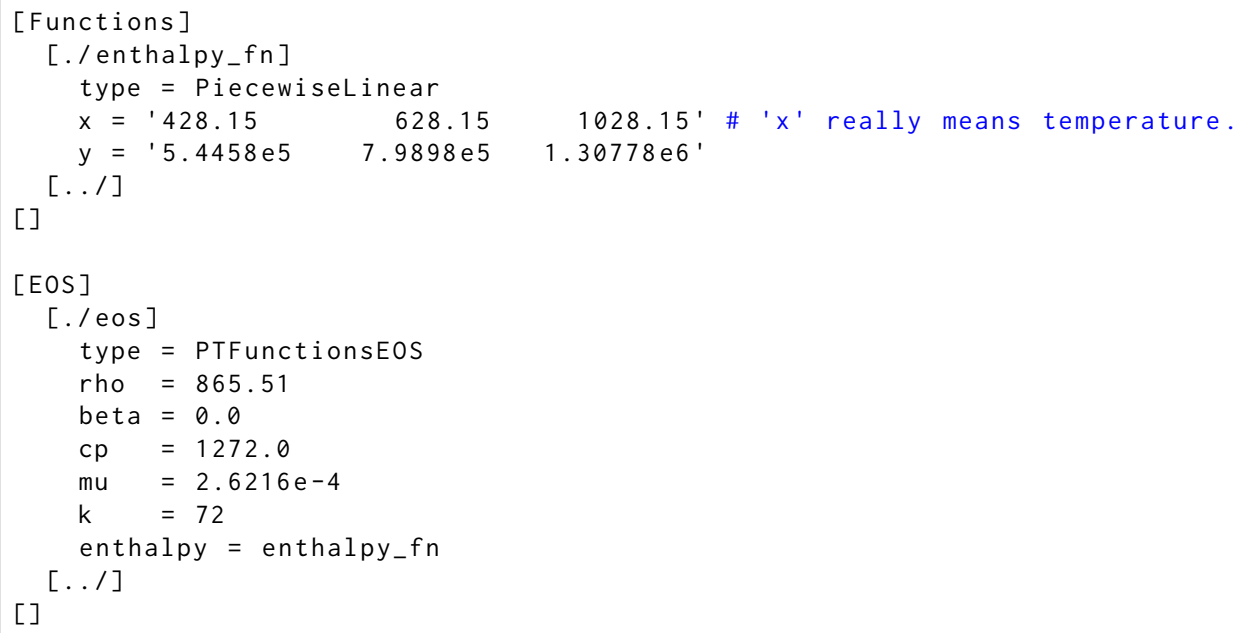

\subsubsection{PTFluidPropertiesEOS}

To take advantages of many existing built-in fluid properties provided within the MOOSE framework, SAM provides an "interface" class, PTFluidPropertiesEOS, to access these fluid property libraries. Its input parameter list is given as follows:

\begin{tabular}{|lll}
\hline [./PTFunctionsEOS] & \\
SC_cp & \# &
\end{tabular}


Other than the four sensitivity coefficients, the only user input is a name pointing to a MOOSEprovided fluid property library. This is a required input parameter:

- $f p$ (required)

This is a required parameter that accepts the name of the user object for a MOOSE-provided fluid library. This user object should have been provided in a separate material properties input block, [MaterialProperties].

An example of PTFluidPropertiesEOS usage is given as:

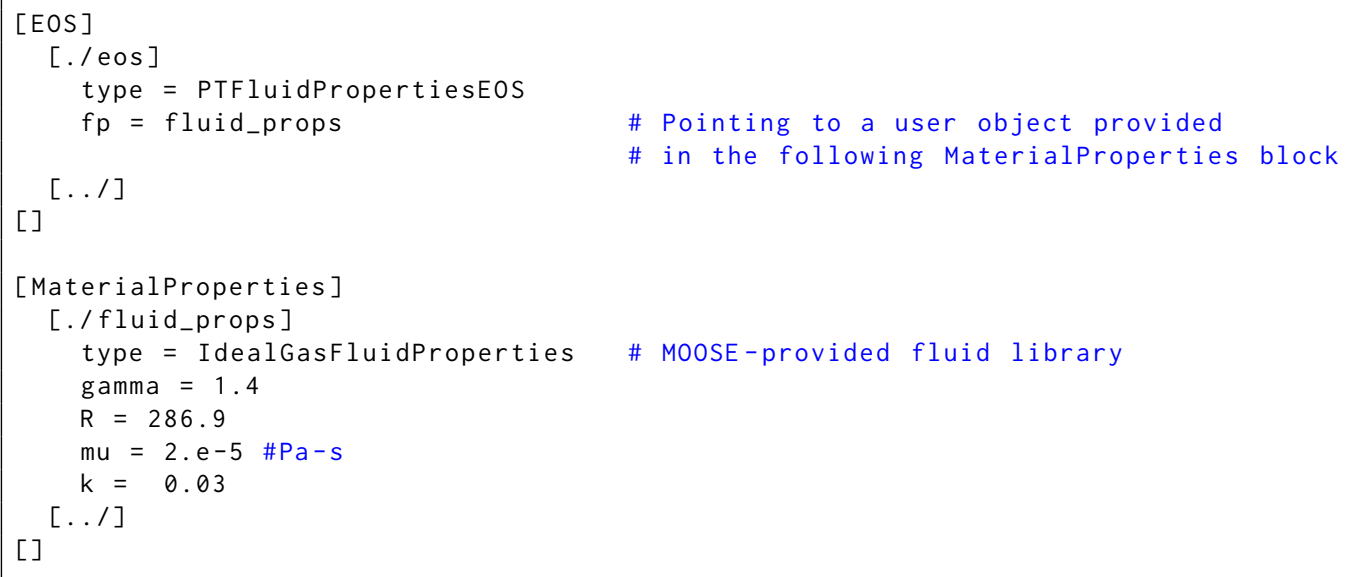

\subsection{Components}

\subsubsection{PBOneDFluidComponent}

PBOneDFluidComponent is the most basic fluid component in SAM. It represents a unit onedimensional (1D) component to simulate the 1D fluid flow in a channel. The geometry parameters such as the hydraulic diameter, flow area, and length, are provided in the input file. The wall friction and heat transfer coefficients can be calculated through the closure models based on flow conditions and geometries or provided by the user input. Internal volumetric heating (or cooling) can be specified by the user input as well. The associated input parameters of the PBOneDFluidComponent Component block are shown below.

\begin{tabular}{|c|c|c|}
\hline \multicolumn{3}{|l|}{ [./PBOneDFluidComponent ] } \\
\hline A & $=($ required $)$ & \# Area of the One-D fluid component \\
\hline $\mathrm{Cgb}$ & $=1$ & $\begin{array}{l}\text { \# Mixing coefficient due to buoyancy } \\
\text { \# and geometry effects }\end{array}$ \\
\hline Cgv & $=($ no_default) & $\begin{array}{l}\text { \# Mixing coefficient due to velocity } \\
\text { \# and geometry effects }\end{array}$ \\
\hline $\mathrm{Dh}$ & $=($ required $)$ & \# Hydraulic diameter \\
\hline HTC_geometry_type & $=$ Pipe & \# Heat transfer geometry type \\
\hline HTC_user_option & $=$ Default & \# Heat transfer correlation user option \\
\hline HT_surface_area_density & $=$ (no_default) & \# Heating surface density \\
\hline HoD & $=1$ & \# wire pitch ratio, height to diameter \\
\hline $\mathrm{Hw}$ & $=$ (no_default) & \# Convective heat transfer coefficient \\
\hline $\mathrm{Ph}$ & $=$ (no_default) & \# Heated perimeter \\
\hline PoD & $=1$ & \# pitch to diameter ratio for parallel bundle \\
\hline SC_HTC & $=1$ & $\begin{array}{l}\text { \# Sensitivity coefficient for HTC } \\
\text { \# multiplicative }\end{array}$ \\
\hline SC_WF & $=1$ & \# Sensitivity coefficient for wall friction, \\
\hline
\end{tabular}




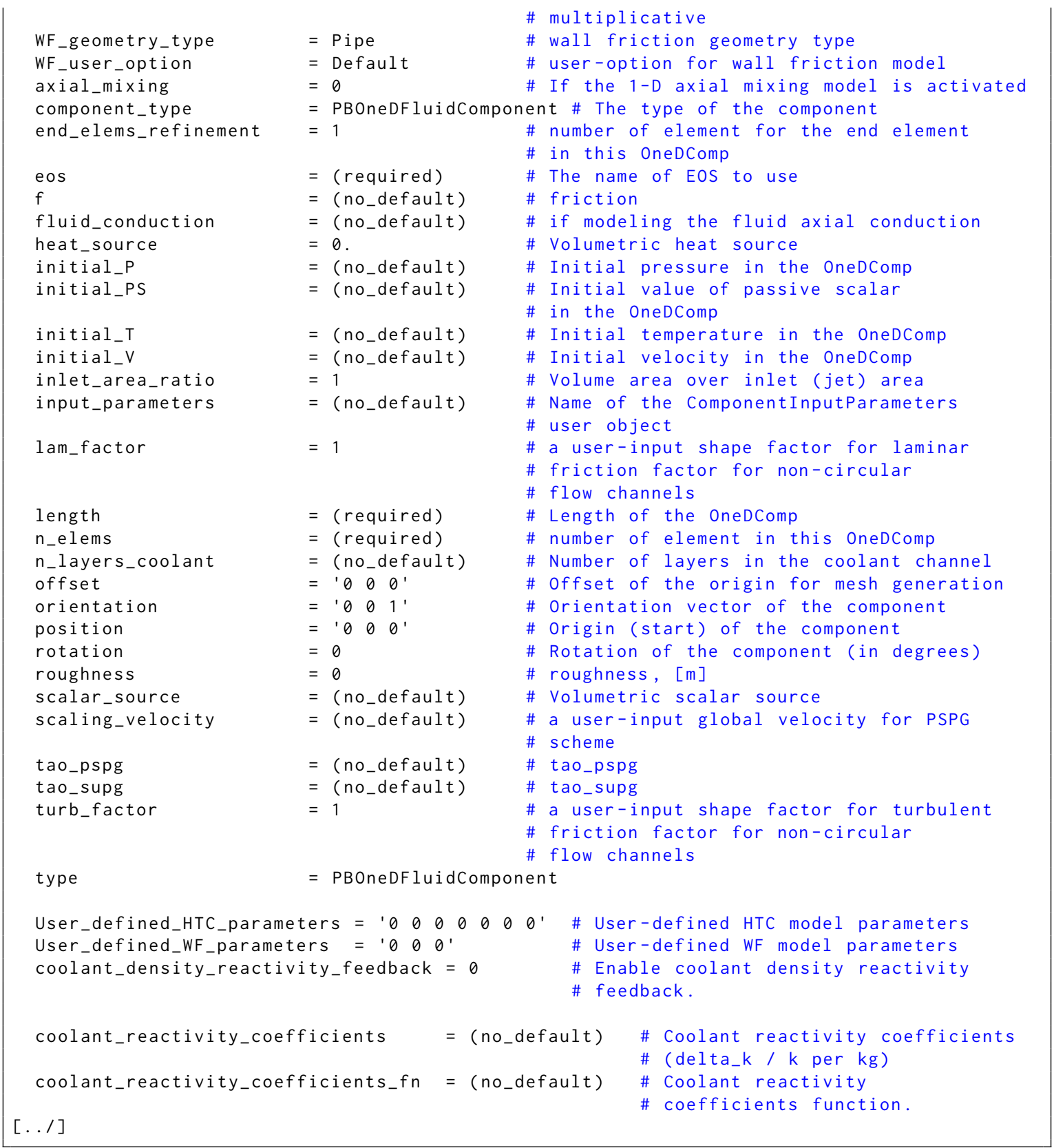




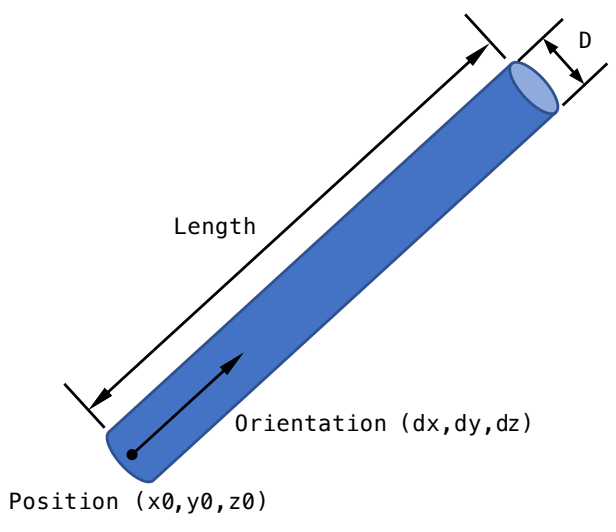

(a) A round pipe

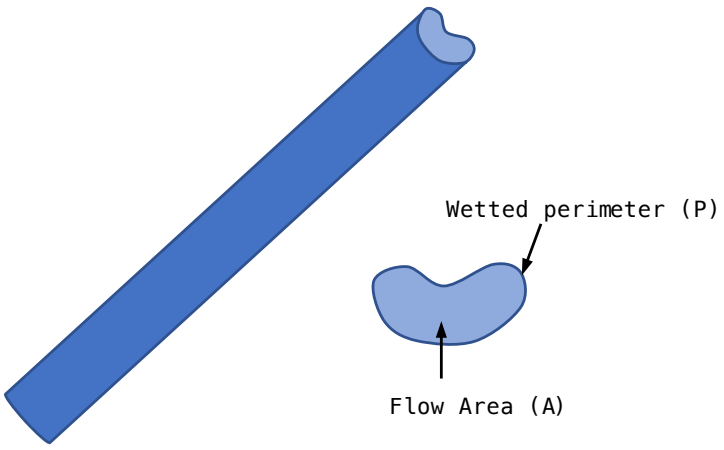

(b) A pipe with irregular cross section

Figure 4.1: SAM PBOneDFluidComponent examples.

Each of the input parameters are discussed as follows. Geometry-related input parameters are discussed first,

- A (required)

Cross-sectional (flow) area of the flow channel. For example, for round pipes, it is simply $\pi D^{2} / 4$, see figure 4.1 .

- length (required)

Length of the flow channel, see figure 4.1.

- position

The origin of the one-dimensional pipe, in $(\mathrm{x} 0, \mathrm{y} 0, \mathrm{z} 0)$, see figure 4.1. The default value is $(0,0$, $0)$, i.e., position $=' 000$ '.

- orientation

The orientation vector of the one-dimensional pipe, in $(\mathrm{dx}, \mathrm{dy}, \mathrm{dz})$, see figure 4.1. Note that it does not have to be a unit vector. The default value is $(0,0,1)$, i.e., orientation $=' \begin{array}{lll}0 & 1\end{array}$ '.

- n_elemes (required)

Number of elements used for the component in the axial direction.

- Dh (required)

Hydraulic diameter of the flow channel. For round pipes, it is simply the pipe diameter; while for flow channels with irregular shape of cross section, it is calculated as:

$$
D_{h}=\frac{4 A}{P}
$$

where $A$ is the cross-sectional area, and $P$ is the wetted perimeter, see figure 4.1. 
- rotation

Rotation of the component (in degrees), which will be used to construct displaced mesh within the code. This is related how SAM internally builds and handles meshes. The default value of this input parameter is 0 , and in most cases, it is safe to leave it unspecified.

- end_elems_refinement

Number of refined elements for the end elements at the begin and end of this component. The default value is 1 , and therefore no refinement. Several examples are shown in figure 4.2 to illustrate how end_elems_refinement works.

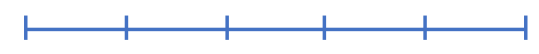

(a) n_elemes=5, end_elems_refinement $=1$ (default)

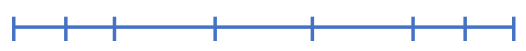

(b) n_elemes=5, end_elems_refinement $=2$

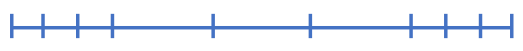

(c) n_elemes $=5$, end_elems_refinement $=3$

Figure 4.2: PBOneDFluidComponent with end element refinements.

- offset

This parameter accepts an offset, in $(\mathrm{dx}, \mathrm{dy}, \mathrm{dz})$, from its origin point, i.e., position, such that the true origin point of the flow channel becomes (origin + offset). Its default value is $(0,0,0)$, meaning no offset at all. This parameter will be depreciated as SAM moves into the real space, instead of the displayed mesh system it is currently using.

Input parameters related to equation of state, and local initial conditions are given as follows:

- eos (required)

The name of equation of state to be used in this component.

- initial_P, initial_V, and initial_T

Local initial condition for pressure, fluid velocity, and temperature, respectively. If specified, these values will override those specified in the global parameter list, and will be used to initialize pressure, fluid velocity, and temperature of this component. If not specified, those global initial values will be used.

- initial_PS

Local initial conditions for passive scalars. If specified, they override values specified in the global parameter list. If not specified, the global initial values will be used. 
The following input parameters are related to how wall frictional coefficients will be calculated in the fluid component,

- $f$

A user-specified constant wall frictional coefficient. If not provided, the wall frictional coefficient will be automatically calculated within the code, see section 4.3 of SAM Theory Manual [1]. Whenever provided, this input parameter will shadow all other wall-friction-related input parameters, such as, WF_user_option, i.e., they will all simply be ignored.

- roughness

Wall roughness. Some wall friction correlations, e.g., the Churchill correlation, require the wall roughness to compute the frictional coefficient. The default value is $0 \mathrm{~m}$.

- WF_geometry_type

Geometry type for SAM to select appropriate wall friction correlations. Currently, there are four types of geometries for selection: 'Pipe' (default), 'WireWrap', 'SquareLattice', and 'Plate', among which, 'WireWrap' is typical for sodium fast reactor designs, and 'SquareLattice' is typical for light water reactor designs.

-WF_user_option

Users can also directly specify wall friction correlations to be used to compute the frictional coefficient, however, it should be noted that some correlations only work with certain geometry type, WF_geometry_type.

The available options for this parameters are: 'Default', 'BlasiusMcAdams', 'ZigrangSylvester', 'Churchill', 'ChengTodreas', and 'User'.

First, if the 'User' option is selected, SAM will compute the wall frictional coefficient from the following Reynolds number-dependent correlation:

$$
f=A+B \times \operatorname{Re}^{C}
$$

and SAM is also expecting an additional input parameter, User_defined_WF_parameters, in which the user-specified constants are given as 'A B C'. This user-specified correlation is to be used in both the laminar and turbulence flow regimes.

For options other than 'User', 'Default' and 'BlasiusMcAdams' are effectively identical: for laminar flow, the Darcy's model will be used, and for turbulent flow, the Blasius correlation is used for Reynolds number smaller than $3 \times 10^{4}$, and the McAdams correlation for Reynolds number larger than $3 \times 10^{4}$.

The 'Churchill' option will use the Churchill model for wall friction coefficient in both the laminar and turbulent flow regimes.

When 'ZigrangSylvester' option is selected, the Zigrang-Sylvester correlation will be used for the turbulent flow regime, while for the laminar flow, the Darcy's model will be used.

When 'ChengTodreas' option is selected, the Cheng-Todreas correlation will be used for both the laminar and turbulent flow regimes. It is also the default option when 'WireWrap' type of geometry is specified, i.e., WF_geometry_type = WireWrap. 


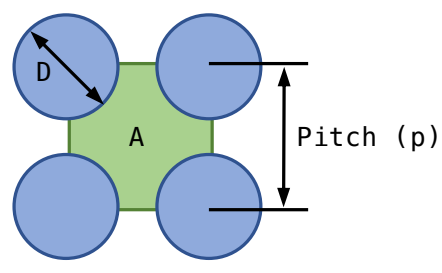

(a) Square-lattice fuel bundle

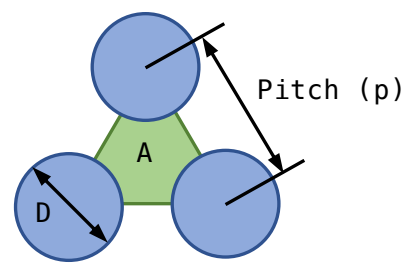

(b) Hexagonal-lattice fuel bundle

Figure 4.3: Fuel bundles in (a) square-lattice, typically seen in light water reactor designs; and (b) hexagonal-lattice, typically seen in sodium fast reactor designs.

Users are referred to section 4.3 of the SAM Theory Manual [1] for more details of the wall friction correlations.

- User_defined_WF_parameters

As discussed in WF_user_option, when WF_user_option = User, this input parameter accepts a set of three values for 'A B C' to compute use-provided wall friction factor. If WF_user_option = User, this input parameter is expected from user input. The default values are ' 000 '.

- PoD

This parameter defines the pitch (p) to diameter (D) ratio in rod bundles, see figure 4.3. This ratio is to be used to compute wall friction factor in, for example, the Cheng-Todreas correlation, and convective heat transfer coefficient in, for example, the Kazimi-Carelli correlation.

- HoD

This parameter defines ratio of "wire lead length" $(\mathrm{H})$ to rod diameter $(\mathrm{D})$, see figure 4.4. Currently, this parameter is only used in the Cheng-Todreas correlation to compute wall friction factor in the wire-wrapped fuel bundle geometry.

- lam_factor and turb_factor

A user-input shape factor for laminar/turbulent flow friction factor for non-circular flow channels. Their default values are both 1.0. Basically, they work as multipliers that are multiplied to the values computed from wall friction correlations other than user-specified constant wall frictional coefficient $f$ and user-specified Reynolds number-dependent correlation WF_user_option.

- SC_WF

This is the same wall friction coefficient multiplier parameter as defined in the global parameter list, section 4.1. If specified in this component, it will override the globally defined parameter locally, i.e., in this component.

The following input parameters are related to wall heat transfer,

- Hw

A user-specified constant wall heat transfer coefficient. If not provided, the wall heat transfer coefficient will be automatically calculated within the code, see section 4.2 of SAM Theory Manual [1]. Whenever provided, this input parameter will shadow all other wall-heat-transfer-related input parameters, such as, HTC_user_option, i.e., they will all simply be ignored. 


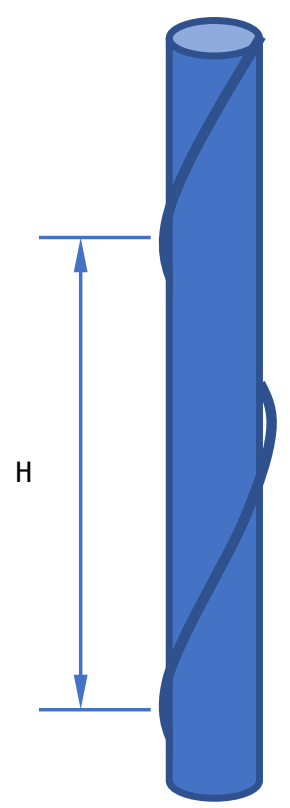

Figure 4.4: Typical SFR wire-wrapped rod configuration.

- $\mathrm{Ph}$

This parameter is the heated perimeter. If heat transfer takes place on the entire wetted perimeter, the heated perimeter is the same as the wetted perimeter, see figure 4.1. For fuel bundles shown in figure 4.3, assuming all fuel rods are heated, the heated perimeters are $\pi D$ and $\pi D / 2$ for (a) square-lattice and (b) hexagonal-lattice fuel bundles, respectively. However, it is not always true that the heated perimeter is the same as the wetted perimeter. If, for example, one of the rod in 4.3 (a) is unheated, the heated perimeter is $3 \pi D / 4$, instead of $\pi D$, which is the value of wetted perimeter.

This is an optional input parameter without a default value given. If specified, it will be used to compute the heat transfer area density, see HT_surface_area_density.

- HT_surface_area_density

This parameter accepts user-specified value for heat transfer surface area density, $a_{w}$, which is heat transfer surface area per fluid volume $\left[\mathrm{m}^{2} / \mathrm{m}^{3}\right]$. In most cases, it is computed as the ratio of heated perimeter to cross-sectional flow area,

$$
a_{w}=\frac{P_{h}}{A}
$$

For a round pipe, as shown in figure 4.1 (a), it is:

$$
a_{w}=\frac{P_{\text {heated }}}{A}=\frac{\pi D}{\pi D^{2} / 4}=\frac{4}{D}
$$

For fuel bundles, as for example shown in figure 4.3 (b), it is:

$$
a_{w}=\frac{P_{\text {heated }}}{A}=\frac{3 \times \frac{\pi D}{6}}{A}
$$


Care should be taken when providing this parameter, as it often depends on how input model is set up. If not specified correctly, often energy imbalance between fluid components and heat structures would be introduced.

As for user input, this is an optional input parameter without a default value given. If the heated perimeter, $\mathrm{Ph}$, is specified, heat transfer area density is computed from its definition, $a_{w}=P_{h} / A$. Users can also specify a constant value for $a_{w}$. If neither heated perimeter nor this parameter is given, it is automatically assumed that the heated perimeter is the same as the wetted perimeter, and thus:

$$
a_{w}=\frac{P_{h}}{A}=\frac{P}{A}=\frac{4}{D_{h}}
$$

in which $P$ is the wetted perimeter.

- HTC_geometry_type

Geometry type for SAM to select appropriate heat transfer coefficient correlations. There are four types channel geometries available in SAM, "Pipe (default)", "Bundle", "Vertical-Plate", and "Horizontal-Plate" .

- HTC_user_option

Similar to wall friction correlation, users can also directly specify correlations to compute heat transfer coefficient. The available options for this parameters are: 'Default', 'NotterSleicher', 'Aoki', 'ChengTak', 'Mikityuk', 'ModifiedSchad', 'GraberRieger', 'McAdams', 'ChurchillChu', 'GaddisGnielinski', 'UserForced' and 'UserNatural'.

If this input parameter is not specified, SAM goes to 'default' options to select appropriate heat transfer coefficient correlations depending on combination of heat transfer geometry, fluid type (liquid metal or not), and flow condition (laminar or turbulent). For pipe geometry, the default correlation for liquid metal $(\operatorname{Pr}<0.1)$ is the Seban-Shimazaki correlation (see section 4.2 of SAM Theory Manual [1]); for fluids other than liquid metal, SAM picks the largest value among those computed from the Dittus-Boelter correlation, the Churchill-Chu correlation, and the correlation for forced laminar flow $(\mathrm{Nu}=4.36)$. For fuel bundle geometry, the default correlation for liquid metal is the same as used for pipe geometry. For non-liquid metal fluids, SAM picks the largest value among those computed from the Inayatov model (modified Dittus-Boelter correlation for fuel bundle geometry), the Churchill-Chu correlation, and the correlation for forced laminar flow $(\mathrm{Nu}=4.36)$.

For pipe geometry, users can also select one of the following correlations, 'NotterSleicher', 'Aoki' or 'ChengTak' for liquid metal, or one from 'McAdams' and 'ChurchillChu' for non-liquid metal fluids. For fuel bundle geometry, available options are 'Mikityuk', 'ModifiedSchad', and 'GraberRieger' for liquid metal; and 'McAdams', 'ChurchillChu', and 'GaddisGnielinski' for non-liquid metal fluids.

SAM also allows users to specify user-defined correlations. Users could select the 'UserForced' option (for forced convection), and then specify a set of 7 numbers, i.e.,

$$
\left[\mathrm{Nu}_{0}, a, b, c, d, e, f\right]
$$

\footnotetext{
${ }^{1}$ Vertical-Plate and Horizontal-Plate have not been treated yet.
} 
in the User_defined_HTC_parameters input parameter, which will be used to compute the Nusselt number in the form of:

$$
\mathrm{Nu}=\mathrm{Nu}_{0}+a\left(\operatorname{Re}^{b}+c\right) \operatorname{Pr}^{d}\left(1+e \operatorname{Re}^{f}\right)^{0.1}
$$

Users could also select the 'UserNatural' option (for natural convection), and then specify a set of 3 numbers in the User_defined_HTC_parameters input parameter,

$$
\left[\mathrm{Nu}_{0}, a, b\right]
$$

which will be used to compute the Nusselt number in the form of:

$$
\mathrm{Nu}=\mathrm{Nu}_{0}+a \mathrm{Ra}^{b}
$$

For heat transfer coefficients, users are referred to SAM theory manual [1] for more details.

- User_defined_HTC_parameters

This input parameter expects either a set of 7 numbers when HTC_user_option $=$ UserForced, or a set of 3 numbers when HTC_user_option = UserNatural (see the previous item). The default values are '0 000000000 '.

- SC_HTC

This is the same heat transfer coefficient multiplier parameter as defined in the global parameter list, section 4.1. If specified in this component, it will override the globally defined parameter locally, i.e., in this component.

Input parameters related to reactivity feedback model are given as follows:

- coolant_density_reactivity_feedback

If specified true, this input parameter enables coolant density reactivity feedback. By default, it is false.

- n_layers_coolant

This parameter specifies the number of layers of coolant in the flow channel. In combination of coolant_reactivity_coefficients or coolant_reactivity_coefficients_fn, the average coolant density in each of these layers will be used to compute the total reactivity feedback in this flow channel. If not specified, it takes the value of number of elements, i.e., n_elems.

- coolant_reactivity_coefficients

This parameter specifies a list of coolant reactivity coefficients. If there is only one value in this list, this value will be used in all layers of coolant to compute total reactivity feedback. Otherwise, the total number of values in this list should be equal to number of layers, i.e., n_layers_coolant (if specified) or n_elems.

- coolant_reactivity_coefficients_fn

The parameter specifies a function (name) to be used to compute coolant reactivity coefficients. The function should be spatially distributed along the channel's axial direction. The reactivity coefficient will be sampled in the middle point of each layer of coolant. 
All other input parameters are discussed as follows:

- tao_supg

An optional input parameter to accept user-specified SUPG stabilization parameter, $\tau_{S U P G}$. If not specified, $\tau_{S U P G}$ is automatically computed within the code. It is not recommended to specify this parameter.

- tao_pspg

An optional input parameter to accept user-specified PSPG stabilization parameter, $\tau_{P S P G}$. If not specified, $\tau_{P S P G}$ is automatically computed within the code. It is not recommended to specify this parameter.

- scaling_velocity

An optional input parameter to accept user-specified reference velocity (magnitude) to compute PSPG stabilization parameter, $\tau_{P S P G}$. If not specified, SAM automatically picks appropriate velocity magnitude to compute $\tau_{P S P G}$. It is not recommended to specify this parameter.

- fluid_conduction

This input parameter overrides the one specified in the global parameter list, which specifies if axial heat conduction effect of the fluid should be included in the fluid energy equation.

- heat_source

This input parameter specifies a direct volumetric heating source to the fluid. A number can be simply specified to assign a constant value as the volumetric heating source. A function name, which must have been given in the [Function] input block, can also be given to this input parameter, so the volumetric heating source will be calculated from this given function.

- scalar_source

This input parameter specifies a list of volumetric sources to the passive scalar variables. Similar to heat_source, both numbers and function names are acceptable options. In addition, numbers and function names could be mixed in the same list.

- axial_mixing

This input parameter specifies if the one-dimensional axial mixing model should be activated. The default value is false, i.e., axial mixing model is not activated.

- inlet_area_ratio

This input parameter specifies the ratio of volume area to inlet (jet) area for the one-dimensional axial mixing model. The default value is 1.0 .

- $\operatorname{Cgv}$

This input parameter specifies the mixing coefficient due to velocity and geometry effects for the one-dimensional axial mixing model. The default value is half of the inlet_area_ratio value. 
- $\mathrm{Cgb}$

This input parameter specifies the mixing coefficient due to buoyancy and geometry effects for the one-dimensional axial mixing model. The default value is 1.0.

- input_parameters

This input parameter is designed to allow SAM input components share common features. For example, in a flow loop consisting of many pipes of the same type, this input parameter allows that these common features (e.g., flow area, hydraulic diameter, etc.) are to be inputted for only once. The details are provided in section 4.4.

An example input block is given as follows:

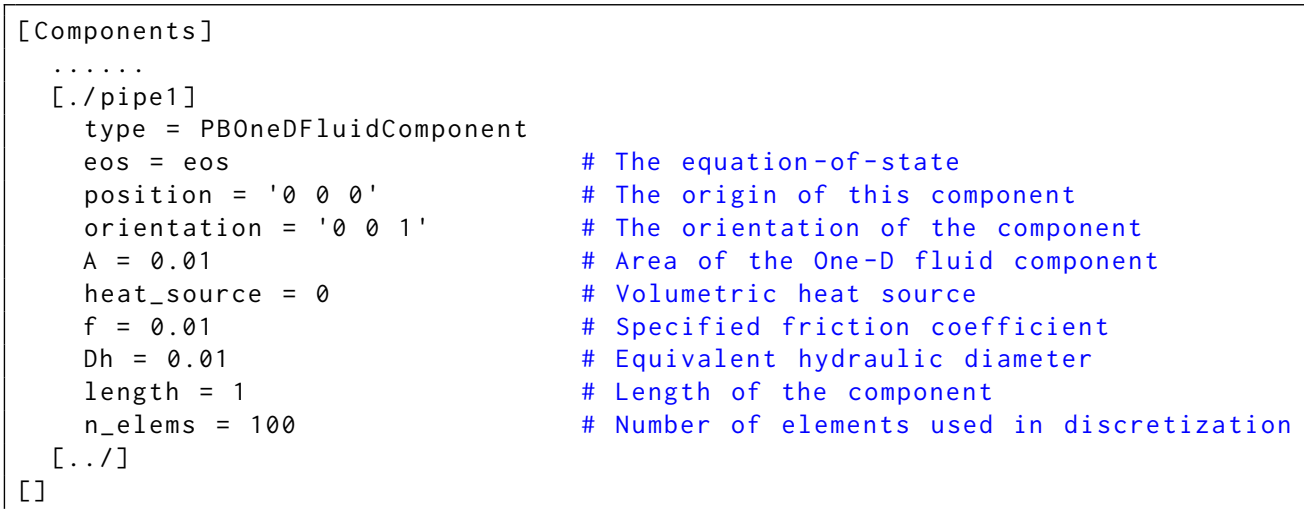

\subsubsection{HeatStructure}

HeatStructure is the most basic solid structure component in SAM. It represents a unit one-D or two-D component in Cartesian or cylindrical coordinates to simulate the heat conduction in solid structures. The geometry parameters such as the thickness and length are provided in the input file. Temperature-dependent solid material properties can be provided in tabular or functional form user-supplied data. Internal volumetric heating can be specified by the user input. Input parameters of HeatStructure is given as follows:

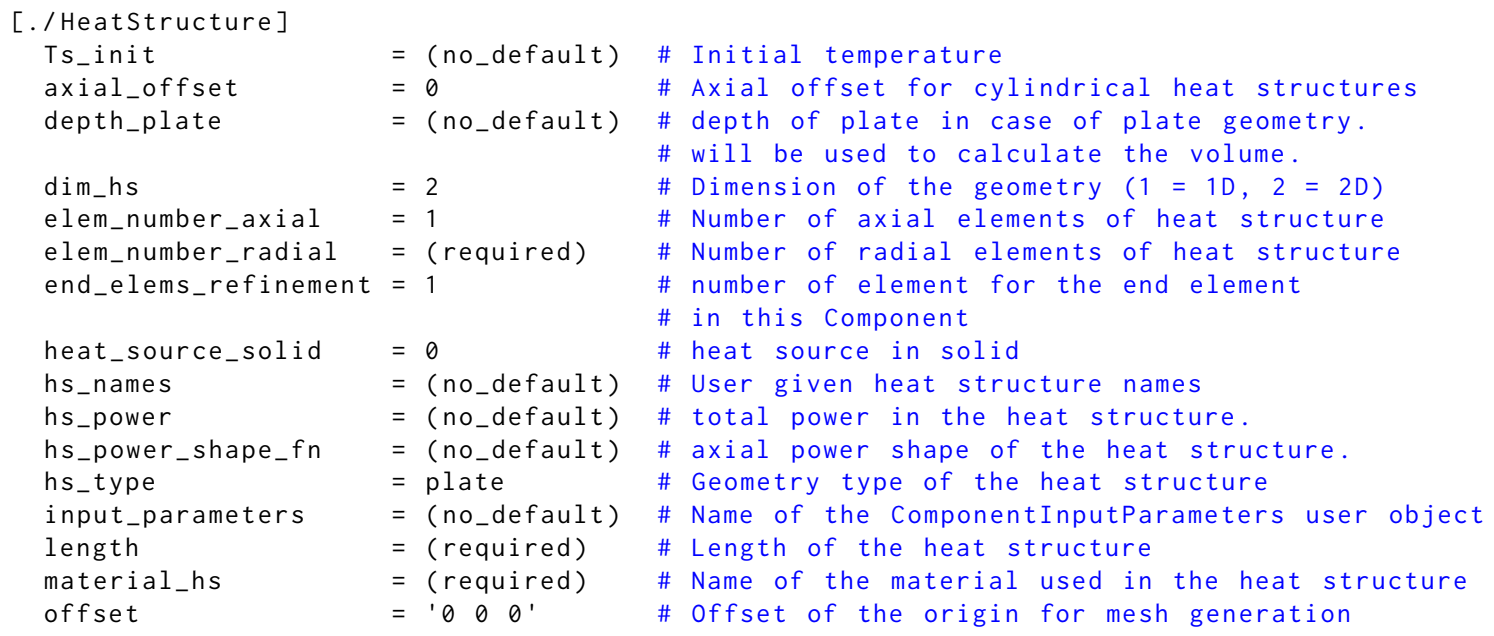




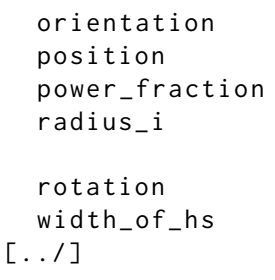

Each of these input parameters are discussed as follows:

- position, orientation, rotation, offset

These input parameters are defined the same way as discussed in section 4.3.1. Also, see figure 4.5 for reference.

- dim_hs

It specifies how the heat structure is modeled, either in one-dimensional (dim_hs $=1$ ) or twodimensional (dim_hs $=2$ ). The default and recommended value is 2, i.e., two-dimensional.

- Ts_init

The initial temperature for the heat structure. If not specified, it seeks the global initial temperature (see global_init_T in section 4.1) as the initial temperature.

- hs_type

Geometry type of the heat structure, which can be either of 'plate' (default) or 'cylinder' type. Note that this input parameter is case insensitive, e.g., 'Plate' is equivalent to 'plate'. An example 2D plate type of heat structure is shown in figure 4.5 .

- hs_names

This input parameter specifies a vector of names for each layer of heat structure. If not specified, SAM automatically creates names for each layer of heat structure. For example, in figure 4.5, the automatically generated names for the two layers would be: $\langle H S$ name $>$ : hs $\theta$ and $\langle H S$ name $>$ : $h s 1$.

- elem_number_axial

Number of elements in the axial direction (along the length direction, see 4.5) of 2-D heat structure, or number of intervals between 1-D heat structures if it is 1-D heat structure. The default value is 1 . In figure 4.5, elem_number_axial $=4$.

- elem_number_radial (required)

This input parameter accepts a vector of numbers that specify the number(s) of elements to be used for each layer of heat structure in the wall-thickness direction. As for example, in figure 4.5, elem_number_radial $=$ ' 34 '.

- width_of_hs (required)

This input parameter specifies a vector of thickness for the layer(s) of heat structure. The size of this input vector should be the same as n_wall_elems. As for example, in figure 4.5, width_of_hs $={ }^{\prime} \delta_{1} \delta_{2}$ '. 


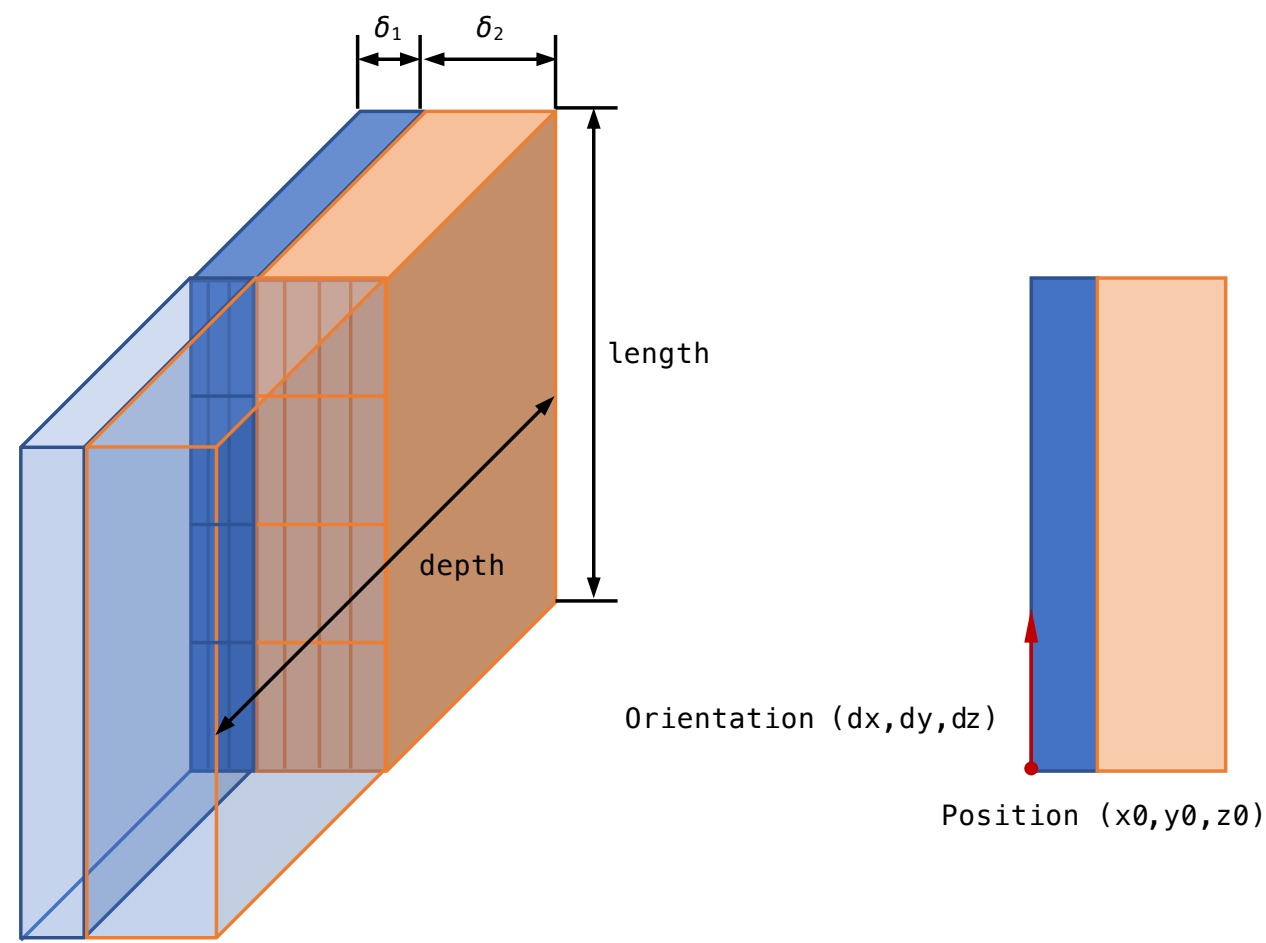

Figure 4.5: An example of two-dimensional plate type of heat structure.

- material_hs (required)

This input parameter specifies a vector of heat structure material name(s) for the layer(s) of heat structure, for example, material_wall = 'SS-304 Wall-Material-2'.

- heat_source_solid

As one of user-specified heat source input options, this input parameter accepts a constant number that is used to specify a uniformly distributed constant volumetric heat source $\left(\mathrm{W} / \mathrm{m}^{3}\right)$ in the entire heat structure. The default value is 0 . If more complex heat source input than this simple constant value is desired, SAM provides other input options, see hs_power.

- hs_power and power_fraction

hs_power specifies the total power, in [W], of the heat structure. If power_fraction is not further specified, it is assumed that the total power is uniformly distributed on the entire heat structure, and therefore, the volumetric heat source is calculated as total power divided by total volume of the heat structure.

In case that power is not uniformly distributed, power_fraction accepts a vector of values that specifies the fraction of the total power for each layer of heat structure. The size of this vector has to be the same as the number of layers in the heat structure. For example, in figure 4.5, one could specify hs_power $=1000$ and power_fraction $=' 0.90 .1{ }^{\prime}$, and thus $90 \%$ of the total power goes to the first layer (to the left), and $10 \%$ goes to the second layer (to the right). Volumetric heat source in each layer of heat structure is then calculated as power in this heat structure layer 
divided by the solid volume of this heat structure layer.

- hs_power_shape_fn

This input parameter accepts a function name, which can be a function of time and/or space. It is important to note that the function value, which could be both temporal and spatial dependent, is multiplied to local volumetric heat source (see previous two items), and there is no re-normalization of total power.

- depth_plate

This input parameter is only required when hs_power is specified and the heat structure type is "plate". It is required to compute the volume of each heat structure layer. For example, in figure 4.5 , the volume of the first heat structure layer is calculated as: Length $\times \delta_{1} \times$ depth.

- radius_i

This input parameter specifies the inner radius of the left-most wall if the heat structure type is cylinder, see figure 4.7 as an example. The default value is 0 , if not specified.

- end_elems_refinement

Number of refinement for the end elements in the beginning and ending of the component (in the axial direction). The default value is 1 . It is only available when the heat structure dimension is two. It usage is similar to the same input parameter for PBOneDFluidComponent, see section 4.3.1, and it is normally used in pair with PBOneDFluidComponent in PBHeatExchanger (see section 4.3.15).

- axial_offset

Axial offset for cylindrical heat structures

- input_parameters (advanced)

This parameter is similar to that of PBOneDFluidComponent, also see section 4.4.

\subsubsection{PBPipe}

In SAM, PBPipe is directly inherited from PBOneDFluidComponent, with the concept to model a one-dimensional pipe flow and its pipe wall with one layer (or several layers) of HeatStructure, as illustrated in figure 4.6. Its input parameters are therefore a superset of input parameters of PBOneDFluidComponent and those to define wall heat structures.

The input parameter subset for PBOneDFluidComponent has been discussed in section 4.3.1. In this section, only the input parameters to define wall heat structures are discussed:

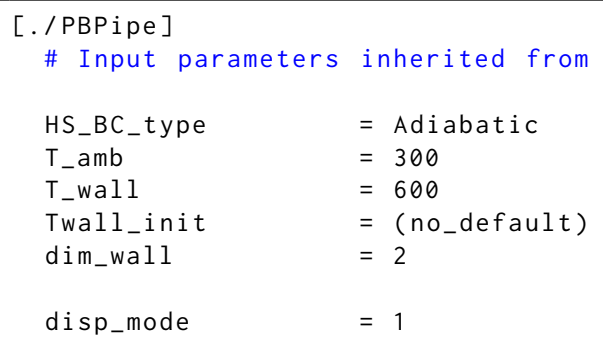




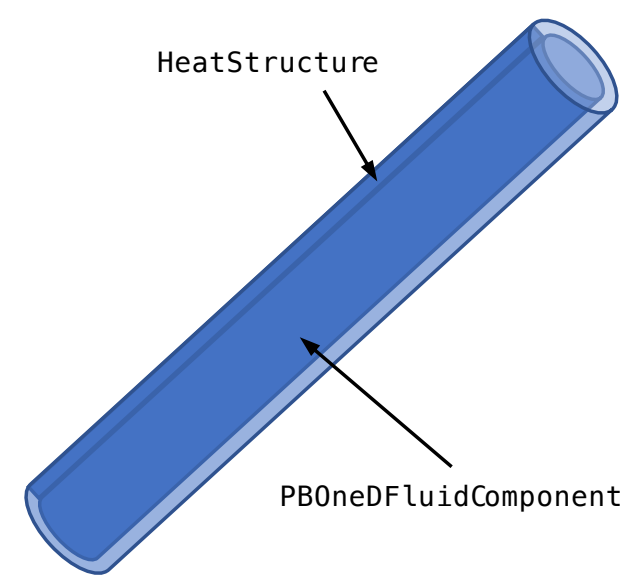

Figure 4.6: SAM's PBPipe component, which consists of a PBOneDFluidComponent to model the one-dimensional fluid flow and one layer (or several layers) of HeatStructure to model its wall.

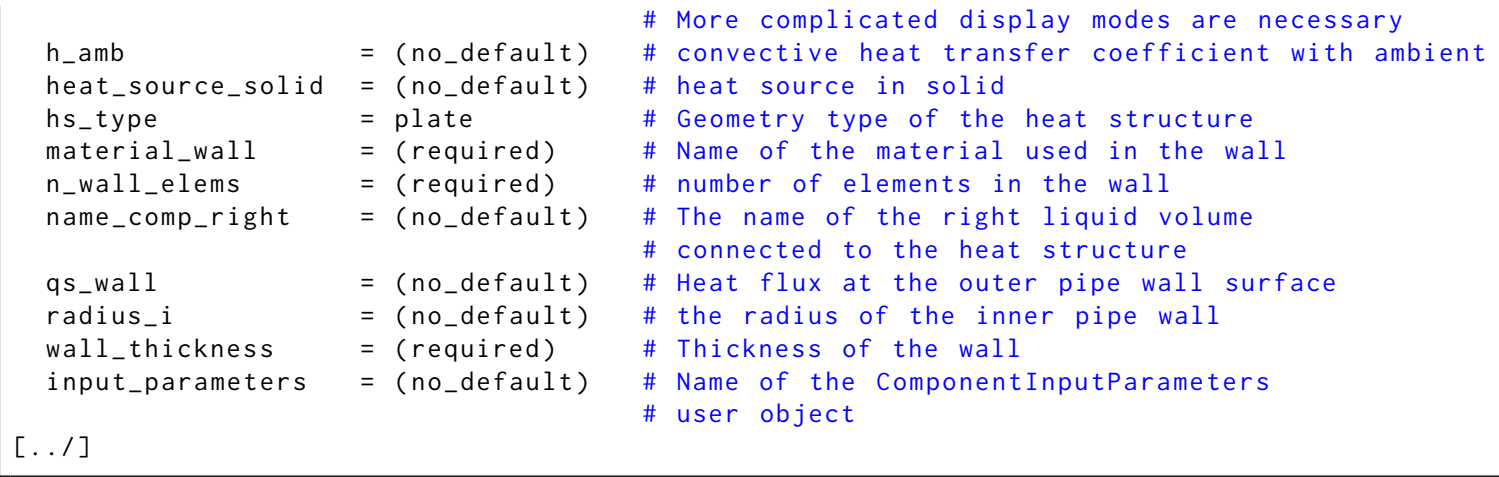

The detailed descriptions of these heat structure-related input parameters are given as follows:

- Twall_init

The initial wall temperature for heat structures. If not specified, it first seeks local initial fluid temperature (see initial_T in section 4.3.1) as initial wall temperature; if local initial fluid temperature is neither given, it then seeks the global initial temperature (see global_init_T in section 4.1) as the initial wall temperature.

- dim_wall

The same as dim_hs in section 4.3.2, it specifies how the wall heat structures are modeled, either in one-dimensional (dim_wall $=1$ ) or two-dimensional (dim_wall $=2$ ). The default and recommended value is 2 , i.e., two-dimensional.

- hs_type

Geometry type of the heat structure, which can be either of 'plate' (default) or 'cylinder' type. Note that this input parameter is case insensitive, e.g., 'Plate' is equivalent to 'plate'. This is the same as hs_type in section 4.3.2.

- radius_i 
This input parameter specifies the inner radius of the pipe wall, if a cylinder type of heat structure(s) is used to model pipe wall, see figure 4.7. If not specified, it takes half of the hydraulic diameter value, i.e., $D_{h} / 2$.

- n_wall_elems (required)

This input parameter accepts a vector of numbers that specify the number(s) of elements to be used for each layer of heat structure in the wall-thickness direction. As for example, in figure 4.7, n_wall_elems $=' 23$ '. This parameter is the same as elem_number_radial in section 4.3.2.

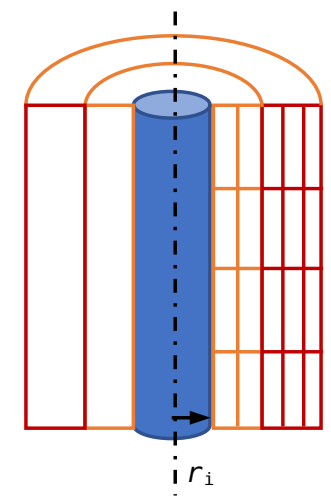

Figure 4.7: An input example of PBPipe with two layers of heat structures to model its wall. For example, it could represent a layer of metal wall and an extra layer of thermal insulation material.

- wall_thickness (required)

This input parameter specifies a vector of wall thickness for the layer(s) of wall heat structure. The size of this input vector should be the same as n_wall_elems. This parameter is the same as width_of_hs in section 4.3.2.

- material_wall (required)

This input parameter specifies a vector of heat structure material name(s) for the layer(s) of wall heat structure, for example, material_wall = 'SS-304 Wall-Material-2'. For obvious reason, the size of this input vector should be the same as n_wall_elems. This parameter is the same as material_hs in section 4.3.2.

- heat_source_solid

This input parameter specifies a vector of volumetric heat source (in numbers) of wall heat structures. The vector size has to be the same as n_wall_elems.

- HS_BC_type

This input parameter specifies the boundary condition type for the pipe outer wall surface. Available options for this parameter are: "Adiabatic (default)", "Temperature", "Convective", and "Coupled".

"Adiabatic", as its name suggests, sets an adiabatic boundary condition for the pipe outer wall surface. 
"Temperature" sets a Dirichlet temperature boundary condition for the pipe outer wall surface. When this boundary condition type is specified, the Dirichlet temperature boundary condition value is also expected from the input file (see T_wall).

"Convective" sets a convective boundary condition to model heat transfer between the pipe outer wall surface and the ambient. Additional input parameters are to be supplied for "Convective" type of boundary condition. This boundary condition type could be supplemented by another two input parameters: user-specified ambient temperature (see T_amb) and user-specified heat transfer coefficient (see h_amb). In addition, a user-specified wall heat flux could be directly given on the pipe outer wall surface (see qs_wall).

"Coupled" sets a conjugate heat transfer boundary condition for the pipe outer wall surface. In this case, the volume component, with which the outer surface transfers heat, has to be specified in the name_comp_right.

- T_wall

Pipe outer wall surface temperature in case that "Temperature" is specified for HS_BC_type. It has to be a number, and the default value for this input parameter is $600 \mathrm{~K}$.

- T_amb and h_amb

When "Convective" is specified for HS_BC_type, T_amb accepts user-specified ambient temperature, and h_amb accepts user-specified heat transfer coefficient. Both input parameters accept either a number or a function name. The default value for T_amb is $300 \mathrm{~K}$.

- qs_wall

When "Convective" is specified for HS_BC_type, besides T_amb and $h_{-}$amb, a user-specified wall heat flux could be directly given to the pipe outer wall surface. It can be either a number of a function name.

- input_parameters

See section 4.4.

\subsubsection{PBCoreChannel}

PBCoreChannel simulates the average coolant flow in rod bundles and heat conduction inside a fuel rod, as well as the convective heat transfer between the coolant and the fuel rod. It is composed of a PBOneDFluidComponent and a HeatStructure. This is also the so-called "Single-Channel" approach to model the fuel assembly. Axial power profiles and the power fractions of total reactor power can be specified for the component. If an outer structure (duct wall) is added to PBCoreChannel, it becomes PBDuctedCoreChannel, which simulates the ducted fuel assemblies as those in SFRs.

When more complex PBCoreChannel is needed to model the reactor fuel assemblies having different axial regions, FuelAssembly or DuctedFuelAssembly are provided in SAM.

From thermal-hydraulics point of view, PBCoreChannel is quite similar to PBPipe, both of which consist of a 1-D fluid flow model and a heat structure, although PBPipe assumes heat structures to be pipe walls, while PBCoreChannel assumes heat structures to be fuel rods, figure 4.8. The major difference between these two components is that PBCoreChannel has a built-in interface to interact with ReactorPower component. It receives power as heat source from the ReactorPower component, 


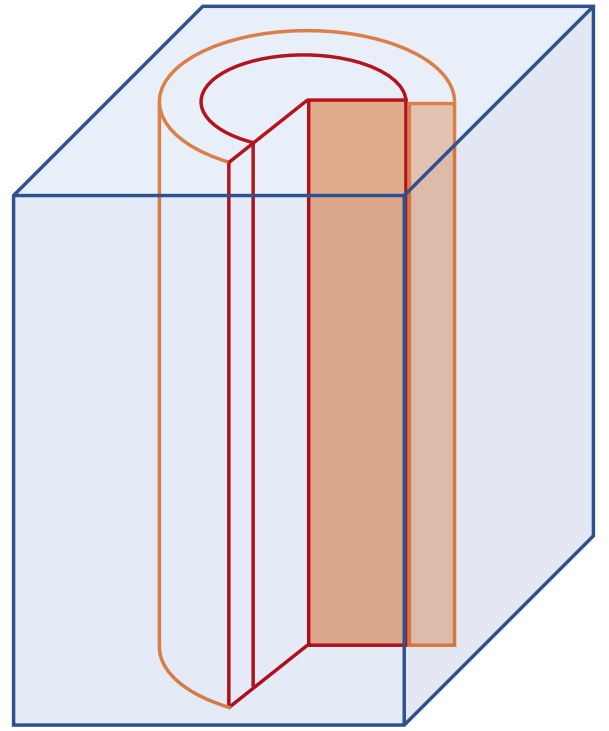

(a)

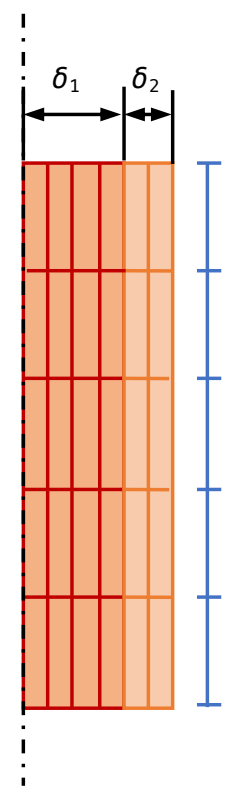

(b)

Figure 4.8: PBCoreChannel component (a) SAM's PBCoreChannel component simulates the average coolant flow in rod bundles and heat conduction inside a fuel rod; and (b) An example mesh used in the PBCoreChannel component, 2-D mesh for heat structure and 1-D mesh for fluid flow.

and also provides the capability to model reactivity feedback. The three types of reactivity feedback mechanisms include fuel's Doppler effect, fuel expansion effect, and coolant density effect (via PBOneDFluidComponent).

The full list of input parameters are given in the following table. Most of them are the same as those for PBOneDFluidComponent (section 4.3.1) or HeatStructure (section 4.3.2).

\begin{tabular}{|c|c|c|c|c|}
\hline \multicolumn{5}{|l|}{ [./PBCoreChannel] } \\
\hline A & $=($ required $)$ & \# & See & PBOneDFluidComponent \\
\hline $\mathrm{Dh}$ & $=($ required $)$ & \# & See & PBOneDFluidComponent \\
\hline HTC_geometry_type & $=$ Pipe & \# & See & PBOneDFluidComponent \\
\hline HTC_user_option & $=$ Default & \# & See & PBOneDFluidComponent \\
\hline HoD & $=1$ & \# & See & PBoneDFluidComponent \\
\hline $\mathrm{Hw}$ & $=$ (no_default) & \# & See & PBOneDFluidComponent \\
\hline $\mathrm{Ph}$ & $=$ (no_default) & \# & See & PBOneDFluidComponent \\
\hline PoD & & \# & See & PBOneDFluidComponent \\
\hline $\mathrm{SC}_{-} \mathrm{HTC}$ & $=1$ & \# & See & PBOneDFluidComponent \\
\hline SC_WF & $=1$ & \# & See & PBOneDFluidComponent \\
\hline end_elems_refinement & $=1$ & \# & See & PBOneDFluidComponent \\
\hline eos & $=($ required $)$ & \# & See & PBOneDFluidComponent \\
\hline$f$ & $=($ no_default) & \# & See & PBOneDFluidComponent \\
\hline fluid_conduction & $=$ (no_default) & \# & See & PBOneDFluidComponent \\
\hline initial_P & $=$ (no_default) & \# & See & PBOneDFluidComponent \\
\hline initial_PS & $=$ (no_default) & \# & See & PBOneDFluidComponent \\
\hline initial_t & $=($ no_default) & \# & See & PBOneDFluidComponent \\
\hline initial_V & $=($ no_default) & \# & See & PBOneDFluidComponent \\
\hline n_elems & $=($ required $)$ & \# & See & PBOneDFluidComponent \\
\hline WF_geometry_type & $=$ Pipe & \# & See & PBOneDFluidComponent \\
\hline WF_user_option & $=$ Default & \# & See & PBOneDFluidComponent \\
\hline lam_factor & $=1$ & \# & See & PBOneDFluidComponent \\
\hline length & $=($ required $)$ & \# & See & PBOneDFluidComponent \\
\hline
\end{tabular}




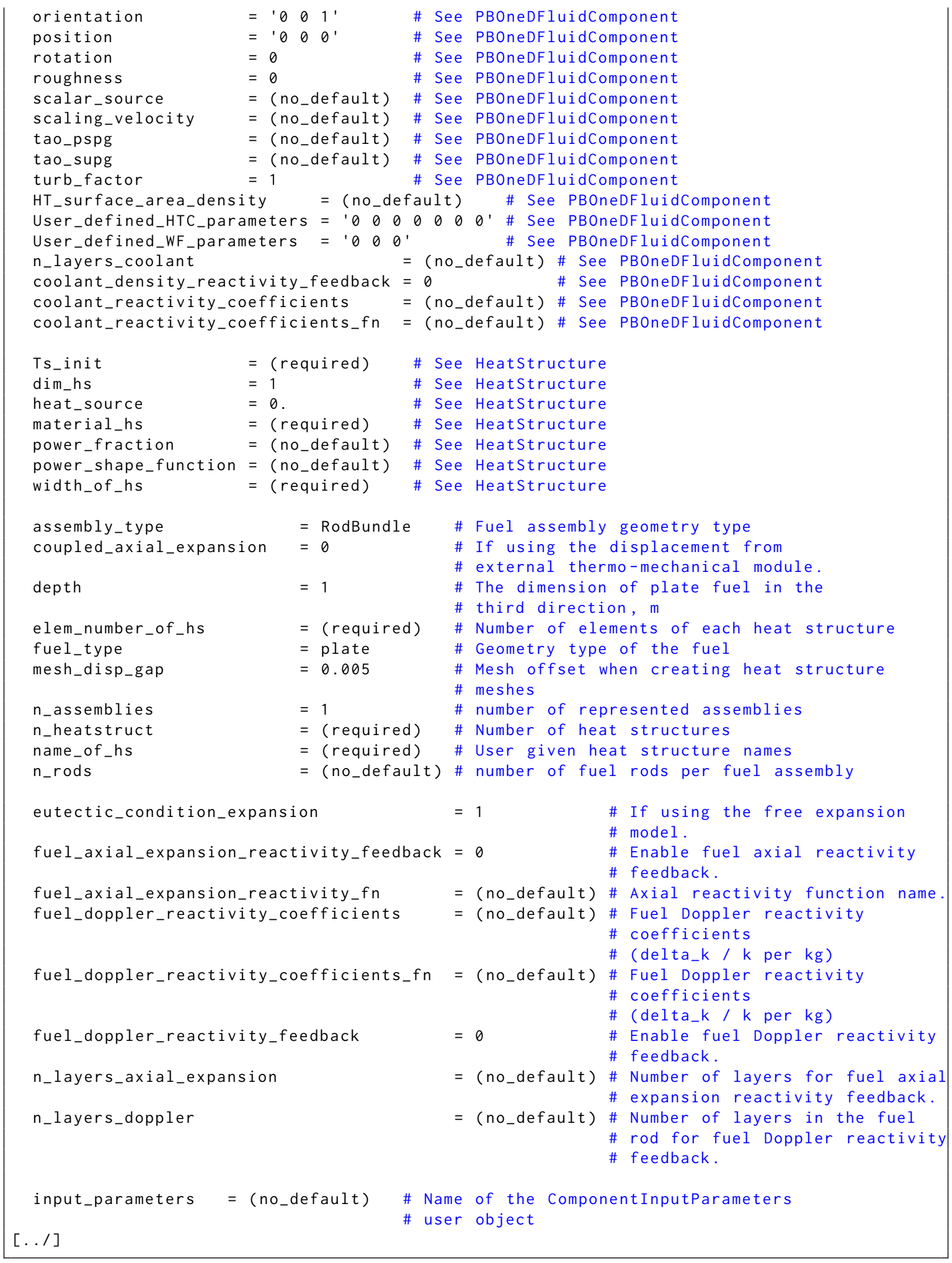

- n_assemblies 
Number of assemblies grouped together that is represented by this PBCoreChannel component.

- elem_number_of_hs (required)

Number of radial elements of heat structure. See elem_number_radial in section 4.3.2.

- Ts_init (required)

The initial temperature for the heat structure, same as Ts_init in section 4.3.2, however this is a required input parameter for PBCoreChannel component.

- n_heatstruct (required)

Number of heat structures. In some cases that no heat structures should be modeled in this component, specify zero to this parameter, and at the same time, specify "None" to fuel_type. When heat structure is modeled, a company component, ReactorPower is expected in the input file, which provides power to this component and accepts reactivity feedback from this component (optional).

- name_of_hs (required)

This input parameter is similar to hs_names of HeatStructure, however, it is a required input parameter for this component. It accepts a vector of names, which specify the name of each heat structure of this component.

- dim_hs

The dimension of the mesh used for the heat structure, same as dim_hs in section 4.3.2. However, the default value here is 1 .

- fuel_type

Same as hs_type in section 4.3.2. It can be "None" if no fuel rod is modeled.

- depth

The depth of plate type of fuels, the same as depth_plate in section 4.3.2

- end_elems_refinement

It is defined, but not used in this component.

- mesh_disp_gap

This input parameter specifies mesh offset in the y-direction, with respect to the fluid component mesh, when creating heat structure meshes. The default value for this parameter is $0.005[\mathrm{~m}]$.

- n_rods

Number of fuel rods per fuel assembly. This parameter is only required when assembly type is "Block-Channel".

- fluid_conduction

It is defined, but not used in this component. 
- assembly_type

This parameter specifies the assembly type of this PBCoreChannel component. Available options include "RodBundle" (default), "Plates", and "Block-Channel". If "Block-Channel" is specified, an additional input parameter $\mathrm{n}_{-}$rods is expected.

- fuel_axial_expansion_reactivity_feedback

This parameter specifies if reactivity feedback due to fuel axial expansion should be considered. By default, it is False (not considered). When it is specified True, additional input parameters are expected from user input (see discussion that follows).

- n_layers_axial_expansion

When fuel_axial_expansion_reactivity_feedback = True, this parameter specifies the number of fuel layers in the axial direction $\left(N_{\text {layer }}\right)$. The length of each of these layers is assumed to be the same, and thus it is equal to total fuel length divided by $N_{\text {layer }}$. If not specified, this parameter takes the number of fluid elements (the same as the number of heat structure elements in the axial direction) as its default value. Averaged axial displacement in fuel rod will be calculated in each of these layers for the component to compute the overall reactivity feedback due to fuel axial expansion.

- fuel_axial_expansion_reactivity_fn

When fuel_axial_expansion_reactivity_feedback = True, this parameter specifies the name of a function that will be used to compute the overall reactivity feedback due to fuel axial expansion.

- coupled_axial_expansion

When fuel_axial_expansion_reactivity_feedback = True, this parameter specifies that, if True, fuel axial expansion is computed from codes external to SAM; and if False (default), it will be calculated internally using SAM's built-in models.

- eutectic_condition_expansion

When fuel_axial_expansion_reactivity_feedback = True, this parameter specifies that, if True (default), eutectic conditions are assumed for fuel and clad expansions; and if False, fuel and clad are assumed to expand freely.

- fuel_doppler_reactivity_feedback

This parameter specifies if reactivity feedback due to fuel's Doppler effect should be considered. By default, it is False (not considered). When it is specified True, additional input parameters are expected from user input (see discussion that follows).

- fuel_doppler_reactivity_coefficients_fn

When fuel_doppler_reactivity_feedback = True, this parameter accepts the name of a function that computes fuel's Doppler effect coefficients. The function can be spatial-dependent only. The value is evaluated in the middle of each fuel layer, see n_layers_doppler.

- fuel_doppler_reactivity_coefficients 
Instead of using a function, it is also possible to directly specify a vector Doppler effect reactivity coefficients for each fuel layer. This vector could contain only single value, such that it will be used for all fuel layers. Otherwise, the number of values in this vector shall be the same as n_layers_doppler.

- n_layers_doppler

Similar to n_layers_axial_expansion, this input parameter specifies the number of fuel layers in the axial direction to compute fuel's Doppler effect, only needed when fuel_doppler_reactivity _feedback $=$ True.

- input_parameters

See section 4.4.

\subsubsection{PBDuctedCoreChannel}

PBDuctedCoreChannel is intended to model a fuel subassembly, which consists of a fuel bundle modeled as a PBCoreChannel and its duct wall modeled as an additional heat structure. Such a ducted fuel subassembly concept is typical in some sodium fast reactor designs. From userinput point of view, PBDuctedCoreChannel inherits all input parameters from the PBCoreChannel component (see 4.3.4), and requires additional input parameters to describe the duct wall, which are listed as follows:

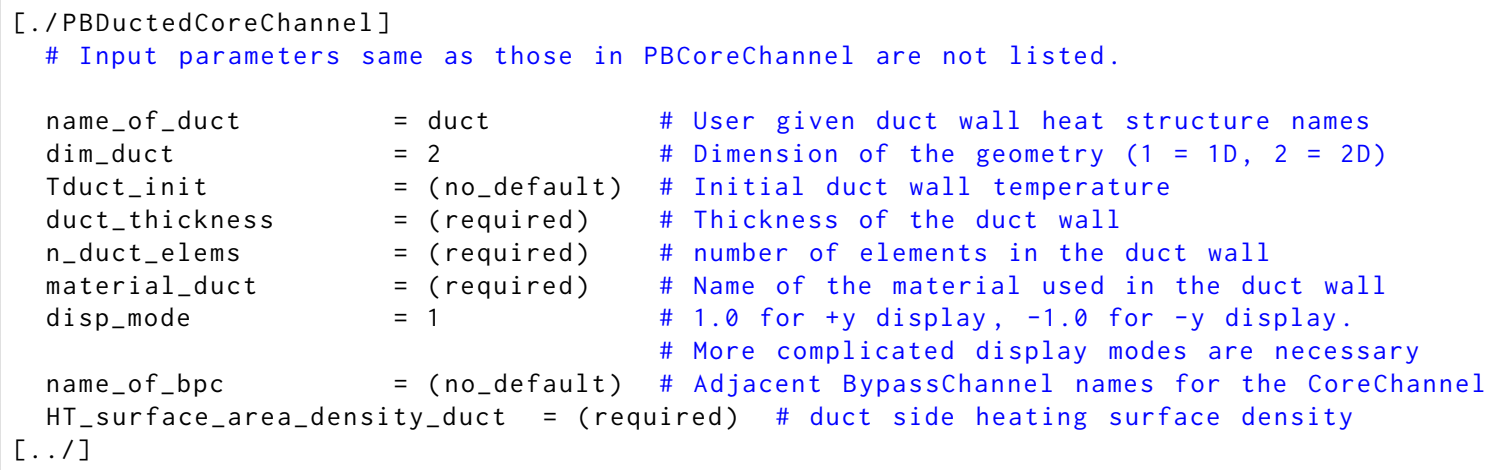

- name_of_duct

This parameter specifies the name of the duct wall. If not specified, the default value is "duct".

- dim_duct

Similar to dim_hs of HeatStructure, it specifies how duct wall heat structure is modeled, either in one-dimensional (1) or two-dimensional (2). The default and recommended value is 2, i.e., two-dimensional.

- Tduct_init

This parameter specifies the initial temperature for the duct wall. If not specified, it takes the global initial temperature, global_init_T (see section 4.1), as the initial duct wall temperature. 
- duct_thickness (required)

The thickness of the duct wall.

- n_duct_elems (required)

Number of elements to model the duct wall in its thickness direction.

- material_duct (required)

This parameter specifies the duct wall material.

- HT_surface_area_density_duct (required)

This parameter specifies the heat transfer surface area density of the duct wall with respect to the CoreChannel.

- disp_mode

To be added.

- name_of_bpc

This parameter specifies the name of BypassChannel adjacent for the CoreChannel.

\subsubsection{PBBypassChannel}

PBBypassChannel is just a PBOneDFluidComponent component with additional physics models. It is designed to model the bypass flow in the gaps between fuel assemblies. It includes the modeling of conjugate heat transfer with the neighboring fuel assembly duct walls. It can also model the direct coolant heating as a fraction of the total reactor power and using the same or different axial power shapes.

From user-input point of view, it inherits all input parameters from PBOneDFluidComponent, and requires additional input parameters to describe its neighboring PBDuctedCoreChannels and direct coolant heating.

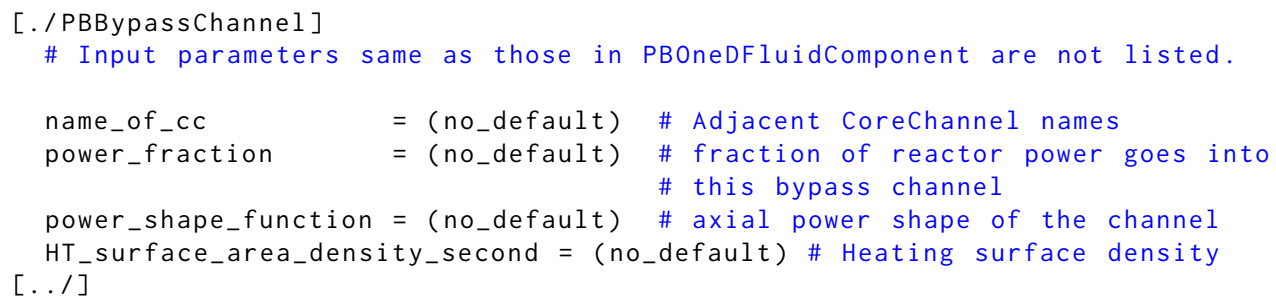

- name_of_cc

This input parameter specifies the names of the two adjacent PBDuctedCoreChannels.

- power_fraction

This input parameter specifies the fraction of reactor power directly goes into this bypass channel. When specified, a ReactorPower component is also expected in the input file that provides the computation of reactor power. 
- power_shape_function

This input parameter accepts a function name, which can be a function of time and/or space. It is important to note that the function value, which could be both temporal and spatial dependent, is multiplied to local volumetric heat source, and there is no re-normalization of total power.

- HT_surface_area_density_second

This input parameter is used in parallel wit HT_surface_area_density inherited from PBOneDFluidComponent. This parameter specifies $a_{w}$ for the second PBDuctedCoreChannels in the list, while HT_surface_area_density specifies $a_{w}$ for the first one.

\subsubsection{PBMoltenSaltChannel}

PBMoltenSaltChannel is a component intended to model the core behavior of molten-salt reactor designs. It is a PBOneDFluidComponent component with additional physics models that account for heating due to reactor power and decay curve.

From user-input point of view, it inherits all input parameters from PBOneDFluidComponent, and requires additional input parameters for the extra physics models.

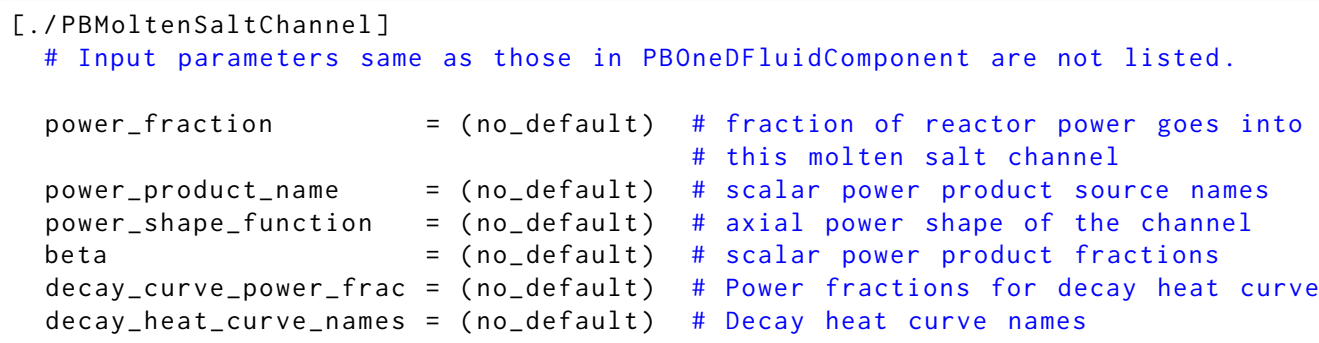

- power_fraction

This input parameter specifies the fraction of reactor power directly goes into this bypass channel. When specified, a ReactorPower component is also expected in the input file that provides the computation of reactor power. If not specified, it is assumed there is no direct heating to this PBMoltenSaltChannel.

- power_shape_function

This input parameter accepts a function name, which can be a function of time and/or space. It is only needed when power_fraction is specified. It is important to note that the function value, which could be both temporal and spatial dependent, is multiplied to local volumetric heat source, and there is no re-normalization of total power. If not specified, SAM takes a default power shape function, which is defined as,

$$
f=\frac{\pi}{2} \sin \left(\frac{\pi x}{L}\right)
$$

where $x$ is the axial location, and $L$ is the channel length. 
- power_product_name

This input parameter specifies a list of passive scalars that this PBMoltenSaltChannel component will model as its fission products. These passive scalars should have been defined in the global parameter, passive_scalar (see section 4.1). The product faction of each scalar is specified in beta. In addition this user-specified list of passive scalars, PBMoltenSaltChannel also has built-in decay curves for several isotopes, see decay_heat_curve_names.

- beta

This parameter specifies the scalar power product fractions.

- decay_heat_curve_names

This component also provides user input to choose built-in decay curves for several isotopes, including "U235T", "PU239T", "U238F", "PU241T", and "TEST235".

\subsubsection{FuelAssembly}

FuelAssembly or DuctedFuelAssembly (see section 4.3.9) model the reactor fuel assemblies composed of multiple PBCoreChannels or PBDuctedCoreChannels, representing different axial regions of a fuel assembly including the active core, gas plenum, lower and upper reflector, lower and upper shield, etc. The junction components (PBSingleJunction) are also auto-created in FuelAssembly or DuctedFuelAssembly to model the connection among the fluid parts of PBCoreChannel or PBDuctedCoreChannel.

The complete list of input parameters of FuelAssembly is give as,

\begin{tabular}{|c|c|c|}
\hline [./FuelAssembly] & & \\
\hline A & $=($ required $)$ & \# Areas of the OneDComp \\
\hline Dh & $=($ required) & \# Hydraulic diameter \\
\hline HTC_geometry_type & $=$ Pipe & \# Heat transfer geometry type \\
\hline HTC_user_option & $=$ Default & \# User option heat transfer correlations \\
\hline HT_surface_area_density & $=($ required $)$ & \# Heating surface density \\
\hline $\mathrm{Hw}$ & $=($ no_default $)$ & \# Convective heat transfer coefficient \\
\hline PoD & $=($ no_default $)$ & $\begin{array}{l}\text { \# pitch to diameter ratio for parallel bundle } \\
\text { \# heat transfer }\end{array}$ \\
\hline $\mathrm{SC}_{-} \mathrm{HTC}$ & $=($ no_default) & \# Sensitivity coefficient for HTC, multiplicative \\
\hline Ts_init & $=($ required $)$ & \# Initial solid temperature \\
\hline dim_hs & $=2$ & \# See PBCoreChannel \\
\hline elem_number_of_hs & $=($ required $)$ & \# Number of elements of each heat structure \\
\hline eos & $=($ required $)$ & \# See PBCoreChannel \\
\hline f & $=($ no_default $)$ & \# friction \\
\hline hs_type & $=($ no_default) & \# Geometry type of the fuel \\
\hline initial_P & $=($ no_default) & \# Initial pressure in the OneDComp \\
\hline initial_t & $=($ no_default $)$ & \# Initial temperature in the OneDComp \\
\hline initial_V & $=($ no_default) & \# Initial velocity in the OneDComp \\
\hline junction_nodalTbc & $=1$ & \# if use nodalTbc for junctions \\
\hline lam_factor & $=($ no_default) & $\begin{array}{l}\text { \# a user-input shape factor for laminar friction } \\
\text { \# factor for non-circular flow channels }\end{array}$ \\
\hline length & $=($ required $)$ & \# Length of the OneDComp \\
\hline material_hs & $=($ required $)$ & $\begin{array}{l}\text { \# Name of the materials used in the heat } \\
\text { \# structures }\end{array}$ \\
\hline $\mathrm{n}_{-}$assemblies & $=1$ & \# See PBCoreChannel \\
\hline $\mathrm{n}_{-} \mathrm{elems}$ & $=($ required $)$ & \# number of element in this OneDComp \\
\hline $\mathrm{n}_{-}$heatstruct & $=($required $)$ & \# Number of heat structures \\
\hline n_zones & $=($ required $)$ & \# number of zones \\
\hline name_of_hs & $=($ required $)$ & \# User given heat structure names \\
\hline orientation & $=10011$ & \# See PBCorechannel \\
\hline
\end{tabular}




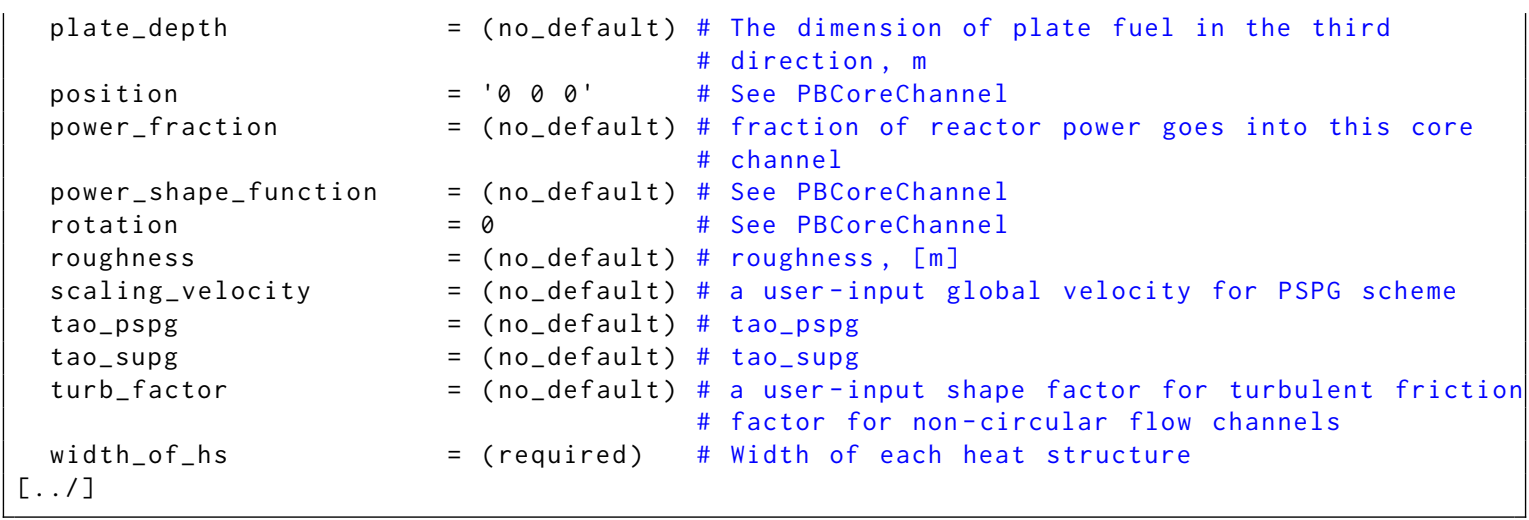

The input parameters of a FuelAssembly are quite similar to those of a PBCoreChannel. However, as a FuelAssembly consists of multiple PBCoreChannels to represent different axial regions of a fuel assembly, most of its input parameters require a list of numbers (or strings), instead of a single number (or string) as in PBCoreChannel. Most of these input parameters could be found in PBCoreChannel or PBOneDFluidComponent and HeatStructure.

- n_zones (required)

This input parameter specifies number or zones, i.e., number of PBCoreChannels, to be used in the FuelAssembly component along the axial direction.

- A (required)

A list of $n_{-}$zones areas for the $n_{-}$zones PBCoreChannels.

- Dh (required)

A list of n_zones hydraulic diameters, $D_{h}$, for the $\mathrm{n}_{-}$zones PBCoreChannels.

- n_elems (required)

A list of $\mathrm{n}_{-} z o n e s$ numbers of elements for the one-dimensional flow channel in the axial direction

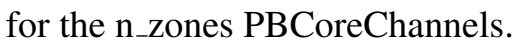

- HTC_geometry_type

A list of $\mathrm{n}_{-}$zones heat transfer geometry types for the $\mathrm{n}$ zones PBCoreChannels. For heat transfer geometry types, see section 4.3.1.

- HTC_user_option

A list of $\mathrm{n}_{-}$zones heat transfer user options for the $\mathrm{n}_{-}$zones PBCoreChannels. For heat transfer user options, see section 4.3.1.

- HT_surface_area_density (required)

A list of $\mathrm{n}_{-}$zones heat transfer surface densities, $a_{w}$, for the $\mathrm{n}_{-}$zones PBCoreChannels.

- Hw

A list of $\mathrm{n}$ zones wall heat transfer coefficients for the $\mathrm{n} \_$zones PBCoreChannels. 
- PoD

A list of $\mathrm{n}_{-}$zones pitch-to-diameter ratios for the $\mathrm{n}_{\text {zzones }}$ PBCoreChannels.

- SC_HTC

A list of $\mathrm{n}_{-}$zones sensitivity coefficients for heat transfer coefficient for the $\mathrm{n}_{-}$zones PBCoreChannels.

- Ts_init (required)

A list of $\mathrm{n}_{-}$zones heat structure initial temperatures for the $\mathrm{n}_{-}$zones PBCoreChannels.

- dim_hs

The dimension of the mesh used for the heat structure of all PBCoreChannels. It does not require a list of dimensions.

- $f$

A list of $\mathrm{n}_{-}$zones user-specified wall frictional coefficient for the $\mathrm{n}_{-}$zones PBCoreChannels.

- hs_type

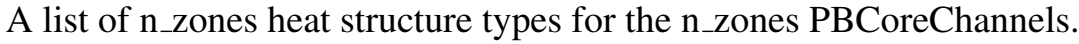

- initial_P, initial_T, and initial_V

A list of $n_{-z o n e s}$ initial pressure (temperature, velocity) for the $\mathrm{n}_{-}$zones PBCoreChannels.

- junction_nodalTbc

As within the FuelAssembly component, $\mathrm{n}$ zones - 1 PBSingleJunctions are automatically created to connect the n_zones PBCoreChannels, this input parameter specifies if 'nodal_Tbc' is to be used for these automatically generated PBSingleJunctions. For 'nodal_Tbc' of PBSingleJunction, see section 4.3.22.

- lam_factor and turb_factor

A list of n_zones user-input shape factors for laminar (turbulent) flow friction factor for noncircular flow channels for the n_zones PBCoreChannels.

- length (required)

A list of $\mathrm{n}_{-} z o n e s$ lengths for the $\mathrm{n}_{-} z o n e s$ PBCoreChannels.

- n_heatstruct (required)

A list of $n_{-}$zones number of heat structures for the $n_{-}$zones PBCoreChannels. Sum all these numbers up, the total number of heat structures, $n_{h s, t o t a l}$, is obtained. For example, in a two-zone FuelAssembly, if $n_{-}$heatstruct $=' 23$ ', there are in total 5 heat structures $\left(n_{h s, t o t a l}=5\right)$ that will require use-inputs for their names, widths, materials, number of elements in its width direction, power fractions, etc. See following discussions.

- elem_number_of_hs (required)

A list of $n_{h s, t o t a l}$ numbers of elements for the $n_{h s, t o t a l}$ heat structures in the FuelAssembly component. 
- material_hs (required)

A list of $n_{h s, t o t a l}$ names of materials for the $n_{h s, t o t a l}$ heat structures in the FuelAssembly component.

- power_fraction (required)

A list of $n_{h s, t o t a l}$ power fractions that goes to the $n_{h s, t o t a l}$ heat structures in the FuelAssembly component.

- plate_depth

A list of $n_{-z o n e s}$ values of the dimension of plate fuel in the third direction for the $\mathrm{n}_{z}$ zones PBCoreChannels. Only needed for plate type of fuels. Also, see section 4.3.4.

- roughness

A list of $\mathrm{n} \_$zones wall roughnesses for the $\mathrm{n}$ zones PBCoreChannels.

- scaling_velocity, tao_pspg, and tao_supg

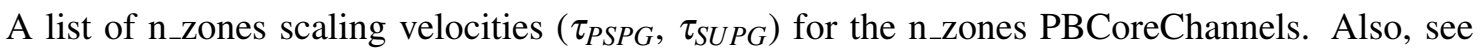
section 4.3.4.

\subsubsection{DuctedFuelAssembly}

DuctedFuelAssembly is simply a FuelAssembly (see section 4.3.8) with an outer duct. It inherits all input parameters from FuelAssembly component, and requires additional input parameters to describe its outer duct:

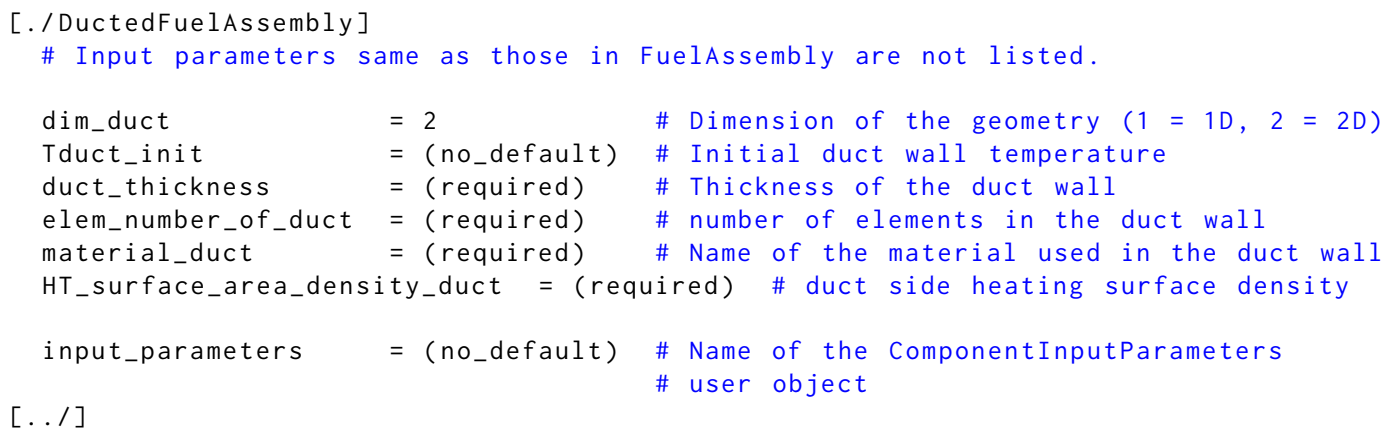

- dim_duct

Similar to dim_hs of HeatStructure, it specifies how duct wall heat structure is modeled, either in one-dimensional (1) or two-dimensional (2). The default and recommended value is 2, i.e., two-dimensional.

- Tduct_init

A list of $\mathrm{n}_{-} z o n e s$ initial temperatures for the duct wall of the $\mathrm{n}_{-}$zones PBDuctedCoreChannels. If not specified, it takes the global initial temperature, global_init_T (see section 4.1), as the initial duct wall temperature. 
- duct_thickness (required)

A list of $\mathrm{n}_{-}$zones duct wall thickness of the $\mathrm{n}_{-}$zones PBDuctedCoreChannels.

- elem_number_of_duct (required)

A list of $n_{z}$ zones numbers of elements, each of which is to be used to specify the number of elements in the duct wall for each PBDuctedCoreChannel.

- material_duct (required)

This parameter specifies the duct wall material for all duct walls.

- HT_surface_area_density_duct (required)

A list of $n_{z}$ zones heat transfer surface area densities of the duct walls with respect to the PBDuctedCoreChannels.

- input_parameters

See section 4.4.

\subsubsection{MultiChannelRodBundle}

To improve the heat transfer between the duct wall and coolant flow, a multi-channel rod bundle model is developed in SAM to account for the temperature differences between the center region and the edge region of the coolant channel in a fuel assembly. Similar approach has been proposed in ENERGY (Yang and Joo, 1999), SAS4A/SASSYS-1 (Fanning 2012), as well as the multi-region porous medium model reported by Yu et al. (2015). The whole fuel assembly can be divided into a number of regions, as shown in figure 4.9. It is quite remarkable that the volumetric heat flux in region 1 is significantly less than that in other regions, based on analytical calculations. Each inner region is modeled as an average core-channel (i.e., a 1-D coolant channel and an average fuel pin). The edge region can be modeled as one core-channel or six core-channels to account for the differences in heat transfer with each side of the duct wall. This zoning strategy is also inspired from the authors' previous experiences in the CFD simulations of the triangle-lattice pin bundles. As shown in the $\mathrm{Hu}$ and $\mathrm{Yu}$ (2016), large temperature gradient were observed in the coolant region near the duct wall, but the temperature distribution elsewhere is very uniform except the hot spots due to the wire-wrap effects. 


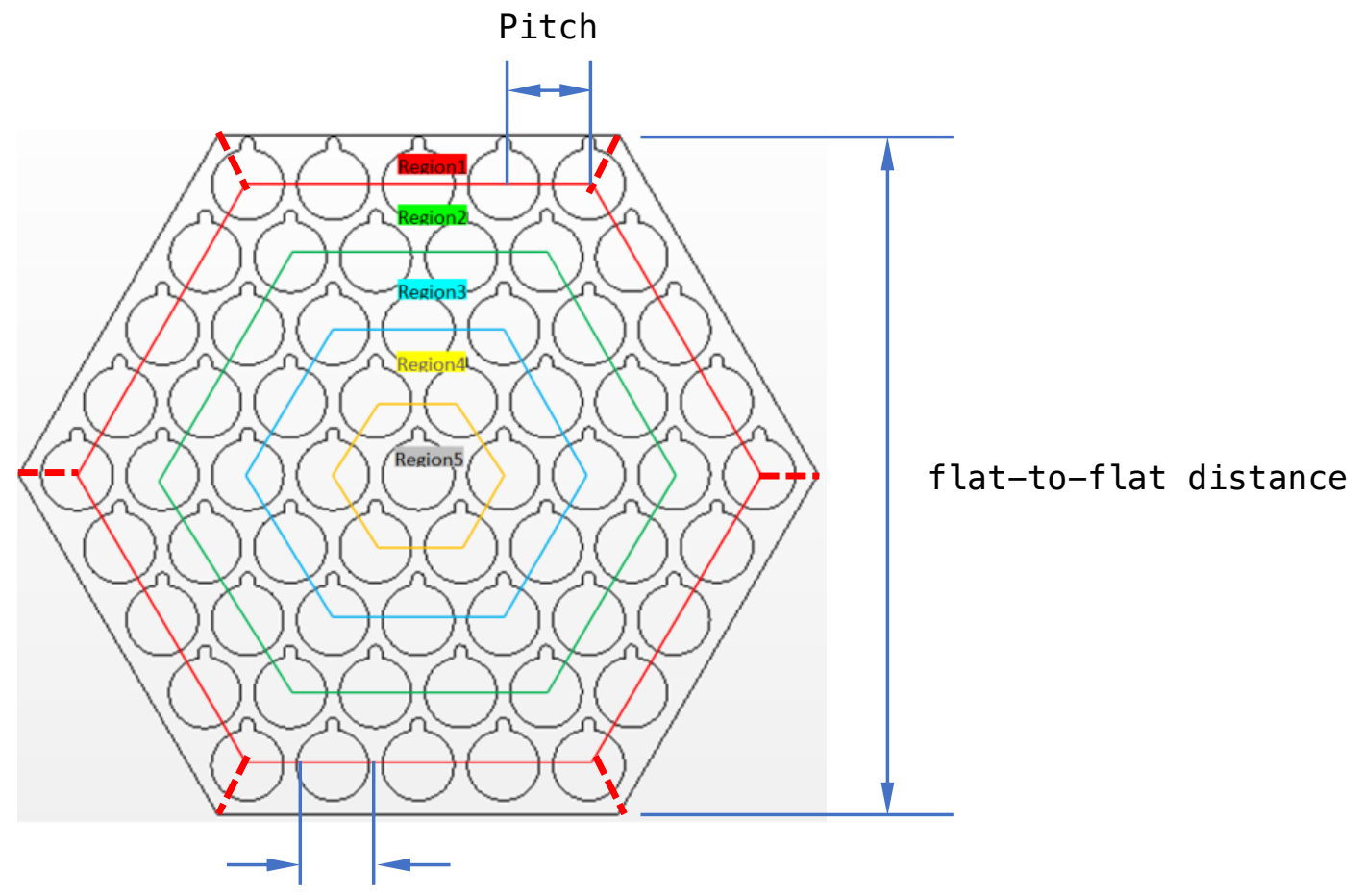

fuel pin diameter

Figure 4.9: Sketch of the regions in the multi-channel model.

In the SAM multi-channel model, the fluid regions are modeled as separate parallel channels with the same pressure drop. For simplicity, it is assumed that there are no mass and momentum exchange between channels. However, the energy exchange is allowed at all axial nodes.

MultiChannelRodBundle assumes that the heat structures are the same for all its automatically generated PBCoreChannels, and thus the same input parameters for heat structure are used for all PBCoreChannels. Also, note that MultiChannelRodBundle does not model the outer duct wall.

Input parameters of MultiChannelRodBundle are given as follows:

\begin{tabular}{|c|c|}
\hline \multicolumn{2}{|c|}{ [./MultiChannelRodBundle] } \\
\hline Ts_init & $=($ required $)$ \\
\hline assem_Dft & $=($ required $)$ \\
\hline beta & $=0$ \\
\hline dim_hs & $=1$ \\
\hline elem_number_of_hs & $=($ required $)$ \\
\hline eos & $=($ required $)$ \\
\hline initial_P & $=$ (no_default) \\
\hline initial_t & $=($ no_default $)$ \\
\hline initial_V & $=($ no_default) \\
\hline lam_factor & $=$ (no_default) \\
\hline length & $=($ required $)$ \\
\hline material_hs & $=($ required $)$ \\
\hline n_channel & $=($ no_default) \\
\hline n_elems & $=($ required $)$ \\
\hline n_heatstruct & $=($ required $)$ \\
\hline n_side & $=($ required $)$ \\
\hline
\end{tabular}

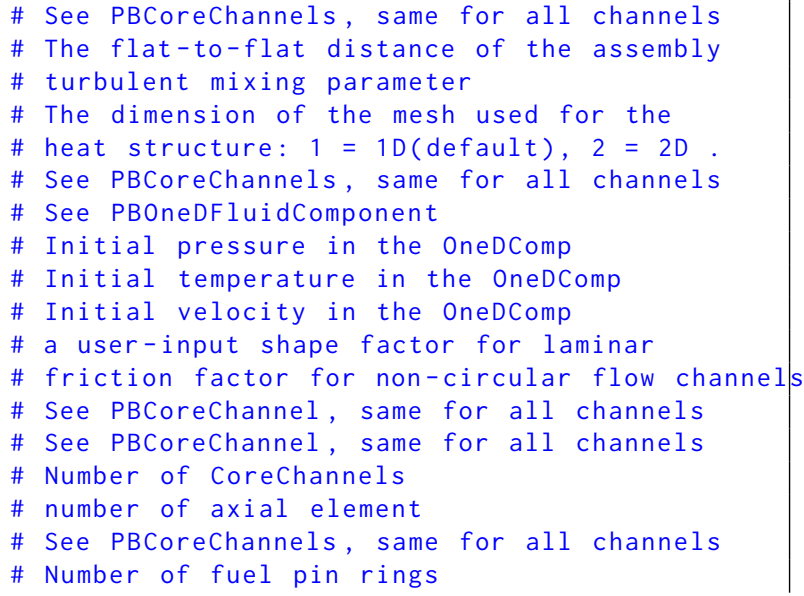




\begin{tabular}{|c|c|c|c|}
\hline n_zones & $=($ required $)$ & \# & Number of zones \\
\hline name_of_hs & $=($ required $)$ & \# & See PBCoreChannels, same for all channels \\
\hline orientation & $=10011$ & \# & See PBOneDFluidComponent \\
\hline pin_diameter & $=($ required $)$ & \# & The fuel pin diameter \\
\hline pin_pitch & $=($ required $)$ & \# & The distance between fuel pin centers \\
\hline position & $=10000^{\prime}$ & \# & See PBOneDFluidComponent \\
\hline power_fraction & $=($ no_default) & \# & See PBCorechannel, same for all channels \\
\hline power_shape_function & $=($ no_default $)$ & \# & See PBCoreChannel, same for all channels \\
\hline radial_power_peaking & $=($ no_default) & \# & radial power peaking factors \\
\hline rotation & $=0$ & \# & See PBOneDFluidComponent \\
\hline roughness & $=($ no_default $)$ & \# & roughness, $[\mathrm{m}]$ \\
\hline scaling_velocity & $=($ no_default) & \# & a user-input global velocity for PSPG scheme \\
\hline tao_pspg & $=($ no_default) & \# & tao_pspg \\
\hline tao_supg & $=($ no_default $)$ & \# & tao_supg \\
\hline turb_factor & $=($ no_default $)$ & $\begin{array}{l}\# \\
\#\end{array}$ & $\begin{array}{l}\text { a user-input shape factor for turbulent } \\
\text { friction factor for non-circular flow channel s }\end{array}$ \\
\hline width_of_hs & $=($ required $)$ & \# & See PBCoreChannels, same for all channels \\
\hline wire_diameter & $=0$ & \# & The wire wrap diameter \\
\hline input_parameters & $=($ no_default $)$ & $\begin{array}{l}\# \\
\#\end{array}$ & $\begin{array}{l}\text { Name of the ComponentInputParameters } \\
\text { user object }\end{array}$ \\
\hline & & & \\
\hline
\end{tabular}

- n_zones (required)

Number of zones (regions) of the MultiChannelRodBundle. For example, n_zones $=5$ in figure 4.9. Note that it is different than the same parameter defined in FuelAssembly (see section 4.3.8), which splits the fuel assembly in the axial direction.

- n_side (required)

Number of fuel pin rings of the MultiChannelRodBundle. As shown in figure 4.9, it is also the same as the number of fuel pins on the side of MultiChannelRodBundle. In figure 4.9, n_side = 5.

- n_channel (required)

Number of core-channels to be modeled in this MultiChannelRodBundle component. It has to be either the same as $n_{-} z o n e s$, such that one core-channel for each zone, or equal to $n_{-} z o n e s+5$, such that the out most zone is modeled as six (6) core-channels and one core-channel for each inner zone. For example, as shown in figure 4.9, if $n_{-}$channel $=5$, one core-channel will be created for each zone (region) 1 to 5 ; however, if $n_{-}$channel $=10$, one core-channel will be created for each zone (region) 2 to 5, while 6 core-channels are to be created for zone (region) 1 . One can easily figure out that MultiChannelRodBundle currently only supports a hexagonal fuel bundles such as the one shown in figure 4.9.

- assem_Dft (required)

This parameter specifies the flat-to-flat distance of the assembly, see figure 4.9.

- beta

Turbulent mixing parameter which will be used to compute the turbulent mixing between neighboring core-channels. Currently, it accepts a simple number for all mixing parameters. This will be improved in future implementations. 
- dim_hs

Same for all core-channels of this MultiChannelRodBundle, it specifies how the heat structure is modeled, either in one-dimensional (dim_hs $=1$ ) or two-dimensional (dim_hs $=2$ ). The default and recommended value is 1 , i.e., one-dimensional.

- initial_P, initial_T, and initial_V

A list of $n_{-}$zones initial pressure (,temperature, and velocity) for the $n_{-}$zones PBCoreChannels.

- lam_factor and turb_factor

A list of n_zones user-input shape factors for laminar (turbulent) flow friction factor for noncircular flow channels for the n_zones PBCoreChannels.

- roughness

A list of $\mathrm{n} \_$zones wall roughnesses for the $\mathrm{n} \_$zones PBCoreChannels.

- scaling_velocity, tao_pspg, and tao_supg

A list of $\mathrm{n}_{z}$ zones scaling velocities $\left(, \tau_{P S P G}\right.$, and $\left.\tau_{S U P G}\right)$ for the $\mathrm{n} z$ zones PBCoreChannels. Also, see section 4.3.4.

- wire_diameter

The wire wrap diameter, same for all PBCoreChannels. The default value is zero.

- pin_diameter (required)

The fuel pin diameter, same for all PBCoreChannels, see figure 4.9.

- pin_pitch (required)

The distance between fuel pin centers, see figure 4.9.

- input_parameters

See section 4.4.

\subsubsection{HexLatticeCore}

HexLatticeCore models a reactor core with a hexagonal lattice such as SFRs, as schematically shown in figure 4.10. It can automatically generate the core lattice of MultiChannelRodBundle or PBCoreChannel components, and the inter-assembly structures (including duct walls and interassembly gaps), based on the geometry information specified in the input. 


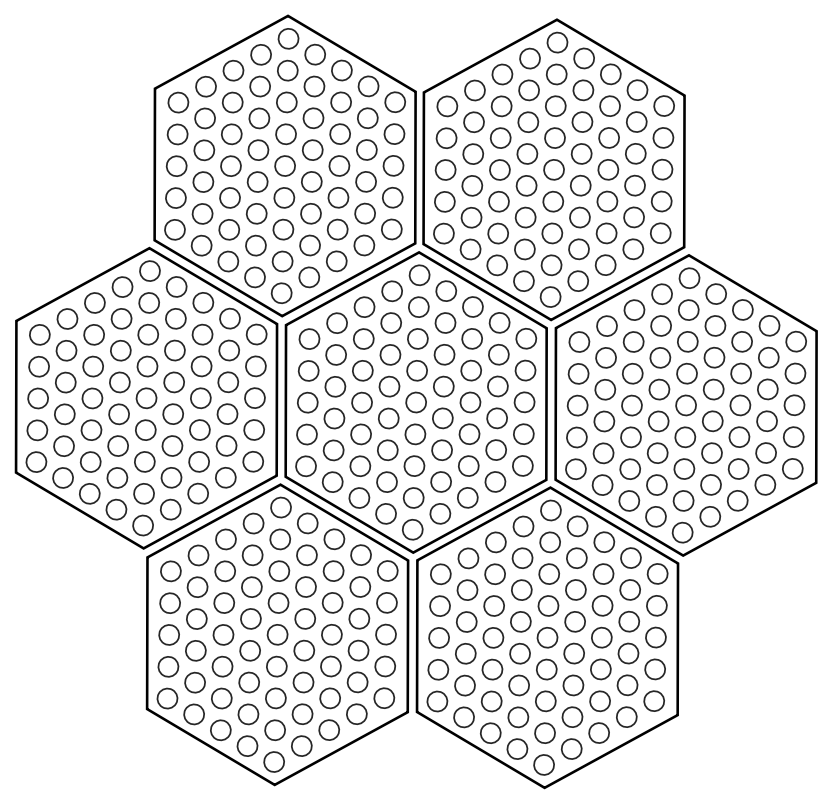

Figure 4.10: Sketch of HexLatticeCore component.

Its input parameters are given as follows:

\begin{tabular}{|c|c|c|}
\hline \multicolumn{3}{|l|}{ [./HexLatticeCore] } \\
\hline assem_Dft & $=($ required $)$ & \# The flat-to-flat distance of the assembly \\
\hline assem_layout & $=($ required $)$ & \# The layout of the assembly lattice \\
\hline assem_pitch & $=($ required $)$ & \# The distance between assembly centers \\
\hline b_multichannel & $=0$ & \# if use MultiChannelRodBundle for each assembly \\
\hline b_radial_heat_transfe & $r=1$ & $\begin{array}{l}\text { \# if modeling radial heat transfer between } \\
\text { \# assemblies }\end{array}$ \\
\hline n_side & $=($ required $)$ & \# Number of side CoreChannels \\
\hline orientation & $=10011$ & \# See PBOneDFluidComponent \\
\hline position & $=10000^{\prime}$ & \# See PBOneDFluidComponent \\
\hline radial_power_peaking & $=($ no_default $)$ & \# radial power peaking factors \\
\hline ref_duct & $=($ no_default) & $\begin{array}{l}\text { \# reference heat structure for one side of an } \\
\text { \# assembly duct wall }\end{array}$ \\
\hline ref_hs & $=($ no_default) & $\begin{array}{l}\text { \# reference heat structure for intra-assembly gap } \\
\text { \# and two duct walls }\end{array}$ \\
\hline $\begin{array}{l}\text { rotation } \\
{[. /]}\end{array}$ & $=0$ & \# See PBOneDFluidComponent \\
\hline
\end{tabular}

- n_side (required)

Number of assemblies on each side of the core. For example, n_side $_{-}=2$ in figure 4.10, and the total number of assemblies in the entire core is 7 .

- assem_Dft (required)

The flat-to-flat distance of the assembly, assumed to be the same for all assemblies in the HexLatticeCore.

- assem_pitch (required)

The distance between assembly centers, assumed to be the same for all assemblies in the HexLatticeCore. 
- b_multichannel

This input parameter specifies if MultiChannelRodBundle (true) or simply PBCoreChannel (false) should be used to represent each assembly in the core. By default, it is 'false', i.e., to use PBCoreChannel.

- assem_layout (required)

This input parameter accepts a list of names for ComponentInputParameters, which are to be used to automatically create all assemblies within the HexLatticeCore component. The number of ComponentInputParameters has to be the same as the number of assemblies in the core, and the assemblies are numbered in an order from left to right, and then top to bottom. If b_multichannel $=$ true, it is expected that the list of ComponentInputParameters are of MultiChannelRodBundleParameters type, otherwise, PBCoreChannelParameters.

- b_radial_heat_transfer

This input parameter specifies if radial heat transfer between assemblies should be modeled. If true (default), HexLatticeCore component automatically adds duct wall heat structures between assemblies, and to the out most boundaries of all assemblies, and models the heat transfer between duct walls/gaps and their neighboring core channels.

- ref_hs

If radial heat transfer between assemblies should be modeled, this input parameter accepts a HeatStructureParameters to build duct wall heat structures between two neighboring assemblies. It is recommended that this reference heat structure models three layers of heat structures, including two duct walls and a layer of gap (modeled as a layer heat structure) between them. It assumes that all such intra-assemblies heat structures have similar geometries.

- ref_duct

If radial heat transfer between assemblies should be modeled, this input parameter accepts a HeatStructureParameters to build duct wall heat structures for the out most boundaries of all assemblies. This reference heat structure should contain only one layer of duct wall. It assumes that all duct wall heat structures have similar geometries.

- radial_power_peaking

This input parameter accepts a list of radial power peaking factors for assemblies within the HexLatticeCore component. The number of power peaking factors has to be the same as the total number of assemblies. The power fraction that goes to each assembly is simply calculated as power peaking factor divided by total number of assemblies. If not specified, it assumes that power is uniformly distributed across all assemblies.

\subsubsection{PBCoupledHeatStructure}

PBCoupledHeatStructure simulates a HeatStructure with controlled boundary conditions at the two surfaces, such as adiabatic, fixed temperature, convective heat transfer with ambient, or coupled with 0-D liquid volume or 1-D liquid components. Normally users will not directly use HeatStructures to create their models, but use PBCoupledHeatStructure instead. 
Most its input parameters are inherited from the base HeatStructure component, see section 4.3.2, and additional input parameters are required to setup its left(right) boundary conditions. The complete input parameters of PBCoupledHeatStructure are listed below:

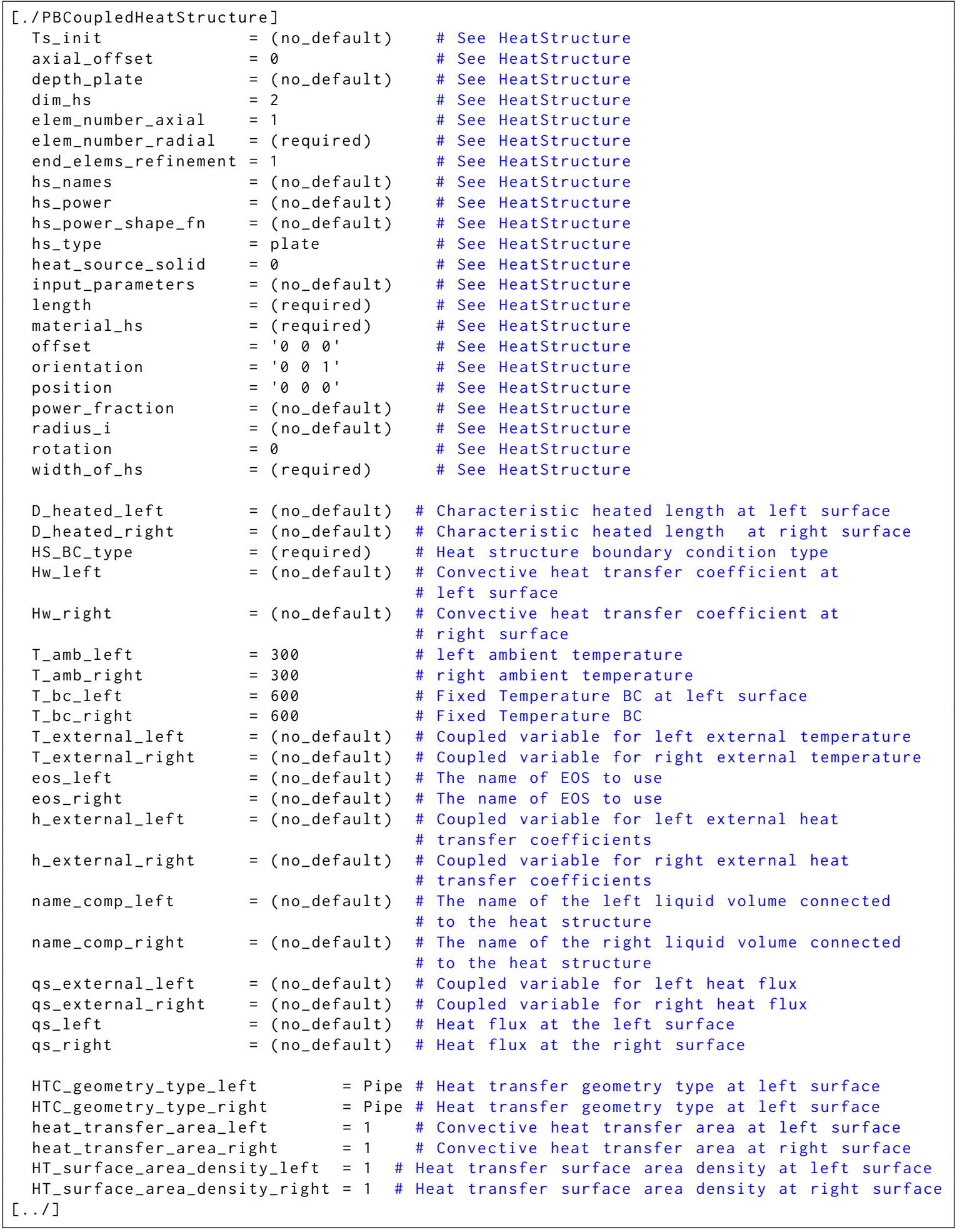




\section{- HS_BC_type (required)}

This input parameter specifies the two boundary condition types for the left and right side of PBCoupledHeatStructure component. Input options include "Temperature", "Convective", and "Coupled".

If "Temperature" is specified, a Dirichlet type of boundary conditions is used, and it expects an additional user-input for the boundary condition temperature, see T_bc_left(right).

If "Convective" is specified, a wall heat flux will be used. The wall heat flux could be directly specified, see qs_left(right). It is also possible to compute the wall surface flux by providing ambient temperature (see T_amb_left(right)) and heat transfer coefficient to the ambient (see Hw_left(right)).

In code coupling computation situations, wall heat flux boundary conditions could be calculated from an external code. Corresponding to the two conditions discussed above, one could specify an externally calculated wall heat flux, see qs_external_left(right); or a combination of external temperature and wall heat transfer coefficient, see T_external_left(right) and h_external_left(right).

If "Coupled" is specified, still a wall heat flux will be used, but the fluid temperature comes from a neighboring component, either a PBVolumeBranch or a PBOneDFluidComponent, see name_comp_left(right). Heat transfer coefficient to the coupled component can be specified in Hw_left(right), and if not specified, they will be automatically computed.

- T_bc_left and T_bc_right

If the left/right boundary condition type is "Temperature", it accepts the value (or a function) for the left/right boundary condition temperatures. The default values are $600 \mathrm{~K}$.

- qs_left and qs_right

If the left/right boundary condition type is "Convective", it accepts the value (or a function) for the left/right wall heat flux as boundary conditions.

- T_amb_left and T_amb_right; Hw_left and Hw_right

If the left/right boundary condition type is "Convective", T_amb_left(right)) specifies the ambient temperature, and Hw_left(right)) specifies the wall heat transfer coefficient to compute the wall heat flux. Both of these inputs could be either values or function names. The default value for T_amb_left(right) is $300 \mathrm{~K}$.

- qs_external_left and qs_external_right

If the left/right boundary condition type is "Convective", it accepts the variable name for externally computed left/right wall heat flux as boundary conditions.

- T_external_left and T_external_right; h_external_left and h_external_right

If the left/right boundary condition type is "Convective", T_external_left(right) specifies an externally computed ambient temperature, and h_external_left(right) specifies an externally computed wall heat transfer coefficient to compute the wall heat flux. Both of these inputs have to be specified as coupled "variables". 
- name_comp_left and name_comp_right

If the left/right boundary condition type is "Coupled", it accepts the name of the component coupled to the left(right) surface of this PBCoupledHeatStructure component. The coupled component has to be either a PBVolumeBranch or a PBOneDFluidComponent.

- eos_left and eos_right

Only when the left/right boundary condition type is "Coupled", and the coupled component is of PBVolumeBranch type, it is required to specify the name of equation of state to the left(right) coupled PBVolumeBranch component.

- D_heated_left and D_heated_right

Only when the left/right boundary condition type is "Coupled", and the coupled component is of PBOneDFluidComponent type, it specifies the characteristic length to compute wall heat transfer coefficient in the coupled left(right) PBOneDFluidComponent component. If not specified, the coupled PBOneDFluidComponent uses its hydraulic diameter as the characteristic length for heat transfer.

- HTC_geometry_type_left and HTC_geometry_type_right

Heat transfer geometry type at the left(right) surface. Acceptable options are "Pipe (default)", "Bundle", "Vertical-Plate", and "Horizontal-Plate".

- HT_surface_area_density_left and HT_surface_area_density_right

Only when the left/right boundary condition type is "Coupled", and the coupled component is of PBOneDFluidComponent type, it specifies the heat transfer surface area density of this PBCoupledHeatStructure component with respect to the coupled left(right) PBOneDFluidComponent component.

- heat_transfer_area_left and heat_transfer_area_right

Only when the left/right boundary condition type is "Coupled", and the coupled component is of PBVolumeBranch type, it specifies the left(right) side heat transfer surface area of this PBCoupledHeatStructure component.

\subsubsection{HeatStructureWithExternalFlow}

HeatStructureWithExternalFlow is also a HeatStructure-based component similar to PBCoupledHeatStructure, however with the main purpose to facilitate code-to-code coupling via its boundary surfaces, either using MOOSE's MultiApp infrastructure or using data exchange with nonMOOSE-based application. When using MOOSE's MultiApp infrastructure, this external MOOSE simulation (a.k.a., MOOSE subApp) will need a different component, HeatTransferWithExternalHeatStructure (see section 4.3.14) to realize data exchange. It is always assumed that its left-side surface is coupled to an external code, while its right-side surface is reserved for SAM to handle its boundary condition.

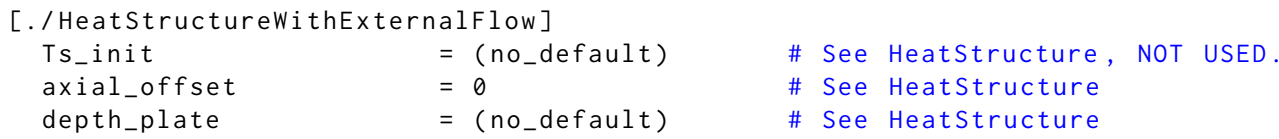




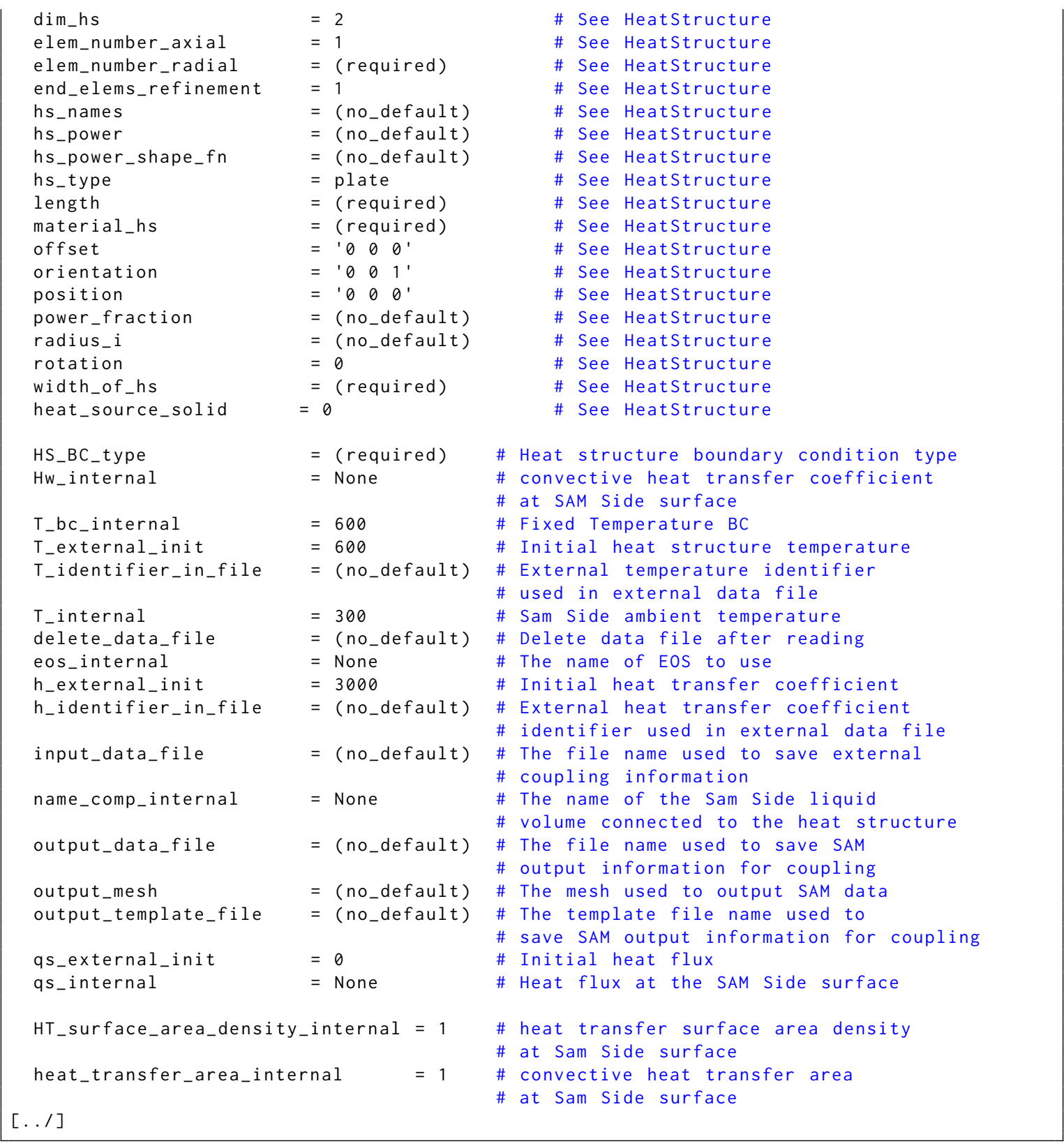

\section{- HS_BC_type (required)}

This input parameter specifies: 1) a list of two heat structure boundary condition types for the left-side (coupled to an external code) and right-side (within SAM) of this component; or 2) a list of one heat structure boundary condition type just for the left-side (coupled to an external code) of this component, while the right-side surface assumes a zero heat flux boundary condition. For the left-side boundary condition type, it could be one of "CoupledConvection_T_h", "CoupledConvection_q", or "PpsConvective".

Using MOOSE's MultiApp infrastructure, "CoupledConvection_T_h" facilitates a surface heat flux coupling via externally computed temperature and heat transfer coefficient, which also re- 
quires additional input parameters, such as:

T_external_init

h_external_init.

Using MOOSE's MultiApp infrastructure, "CoupledConvection_q" simply takes an externally computed wall heat flux as its boundary condition. It requires an additional input parameter, qs_external_init.

"PpsConvective" facilitates SAM code coupling with a non-MOOSE-based code, such as SAS. The coupling mechanism is similar to "CoupledConvection_T_h", which also computes its wall heat flux from externally computed fluid temperature and heat transfer coefficient. Additional input parameters are required for these external fluid temperature (see T_identifier_in_file) and heat transfer coefficient (see h_identifier_in_file).

The right-side boundary condition type of this heat structure can be "Temperature", "Convective", or "Coupled", the same as defined in PBCoupledHeatStructure component, see section 4.3.12.

- T_external_init

This input parameter specifies the initial value for the external temperature variable. The default value is $600 \mathrm{~K}$.

- h_external_init

This input parameter specifies the initial value for the external heat transfer coefficient variable. The default value is $3000 \mathrm{~W} / \mathrm{m}^{2} \mathrm{~K}$.

- qs_external_init

This input parameter specifies the initial value for the externally computed wall heat flux variable. The default value is 0 .

- input_data_file, T_identifier_in_file, and h_identifier_in_file

When "PpsConvective" is used, input_data_file specifies the file name, where the external fluid temperature and heat transfer coefficient are stored. T_identifier_in_file specifies the name of external fluid temperature stored in this file. h_identifier_in_file specifies the name of external heat transfer coefficient stored in this file.

- delete_data_file

This input parameter specifies if the external input file (see input_data_file) should be deleted after data being extracted from it.

- output_data_file, output_mesh, output_template_file

When coupled to an external code, such as SAS, these input parameters specify the output file name to store SAM's out-going data, the mesh to be used for SAM's out-going data, and the template file that this out-going data file should follow.

- T_bc_internal

If the right-side surface boundary condition type is "Temperature", this input parameter specifies the surface temperature value (or a function name). 
- T_internal and Hw_internal

If the right-side surface boundary condition type is "Convective", as one of two available options, this input parameter specifies the fluid temperature and heat transfer coefficient values. The other option is to specify qs_internal.

- qs_internal

If the right-side surface boundary condition type is "Convective", this input parameter specifies the surface flux value (or a function name).

- name_comp_internal and eos_internal

If the right-side surface boundary condition type is "Coupled", these input parameters specify the name of, and equation of state used in this coupled component.

- HT_surface_area_density_internal

If the right-side surface boundary condition type is "Convective", and the coupled component is of one-dimensional flow type, such as PBOneDFluidComponent and PBCoreChannel, this input parameter specifies the heat transfer area density of this heat structure. The default value is 1 $\left[\mathrm{m}^{2} / \mathrm{m}^{3}\right]$ (or $\left.[1 / \mathrm{m}]\right)$.

- heat_transfer_area_internal

If the right-side surface boundary condition type is "Convective", and the coupled component is of zero-dimensional flow type, such as PBVolumeBranch, this input parameter specifies the heat transfer area of this heat structure. The default value is $1\left[\mathrm{~m}^{2}\right]$.

\subsubsection{HeatTransferWithExternalHeatStructure}

This is a non-geometric type of component to facilitate data exchange between two MOOSEbased simulations via its MultiApp infrastructure. This component is intended to take the wall temperature from an external MOOSE-based application (i.e., a master mooseApp), while export its fluid component's fluid temperature and heat transfer coefficient to this external application, in order to compute the conjugate heat transfer.

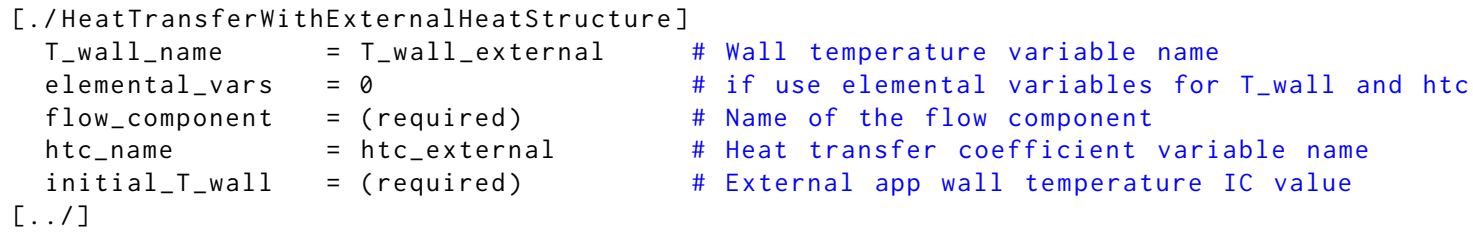

- flow_component (required)

The name of the flow component, to which an external HeatStructure is coupled with, i.e., where the external heat flux to be applied.

- T_wall_name

The name of incoming external wall temperature name, which will be transferred from an external MooseApp. Their default values are "T_wall_external". 
- initial_T_wall (required)

This input parameter specifies the initial value for the externally computed wall temperature before data transfer begins.

- htc_name

The name of outgoing heat transfer coefficient name, which is computed in the flow component (see flow_component) and to be transferred to the external MooseApp. Their default values are "T_wall_external".

- elemental_vars

This input parameter specifies if elemental type of variables, in contrast to nodal type of variable, are to be used for the outgoing heat transfer coefficient and the incoming external wall temperature.

\subsubsection{PBHeatExchanger}

PBHeatExchanger simulates a heat exchanger, including the fluid flow in the primary and secondary sides, convective heat transfer, and the heat conduction in the tube wall. Both countercurrent and concurrent heat exchangers can be modeled. The two sides of the heat exchanger can have different orientation, lengths, flow areas, and hydraulic diameters. This gives the users more flexibilities to model a generic heat exchanger, including advanced heat exchangers being pursued by advanced reactor designs. Note that the two fluid sides of the heat exchanger and the tube wall must have the same number of elements axially.

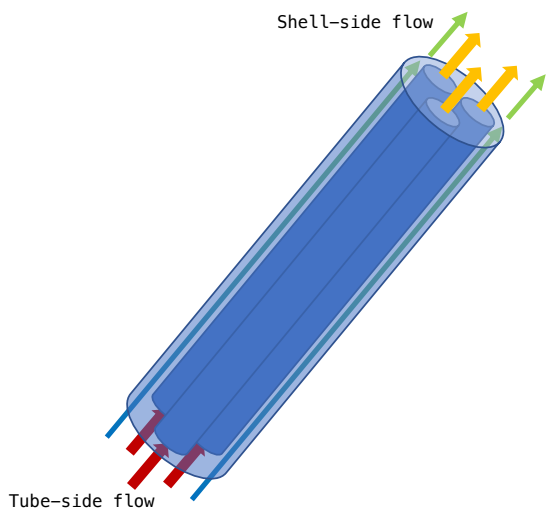

(a) Concurrent design

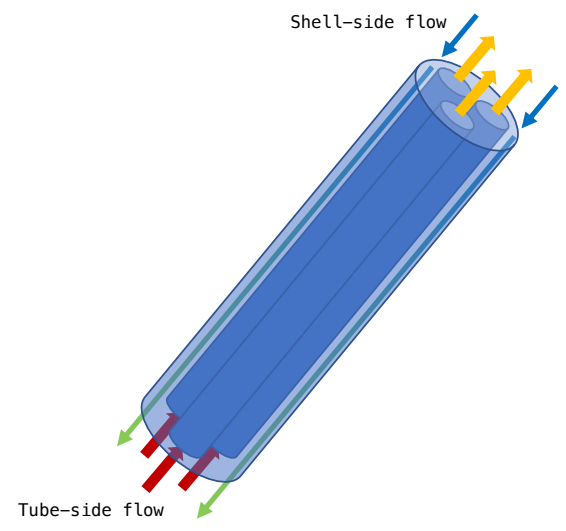

(b) Counter-current design

Figure 4.11: Two types of PBHeatExchanger component designs. As an example, the two figures show shell-and-tube heat exchanger design.

The input parameters of PBHeatExchanger include those to setup the primary and secondary flow pipes, as well as those to setup the heat structure between the two pipes. Most of them are referred to PBOneDFluidComponent (see section 4.3.1) or HeatStructure (see section 4.3.2).

[. / PBHeatExchanger ]

HX_type

= Countercurrent

\# Heat exchanger type 


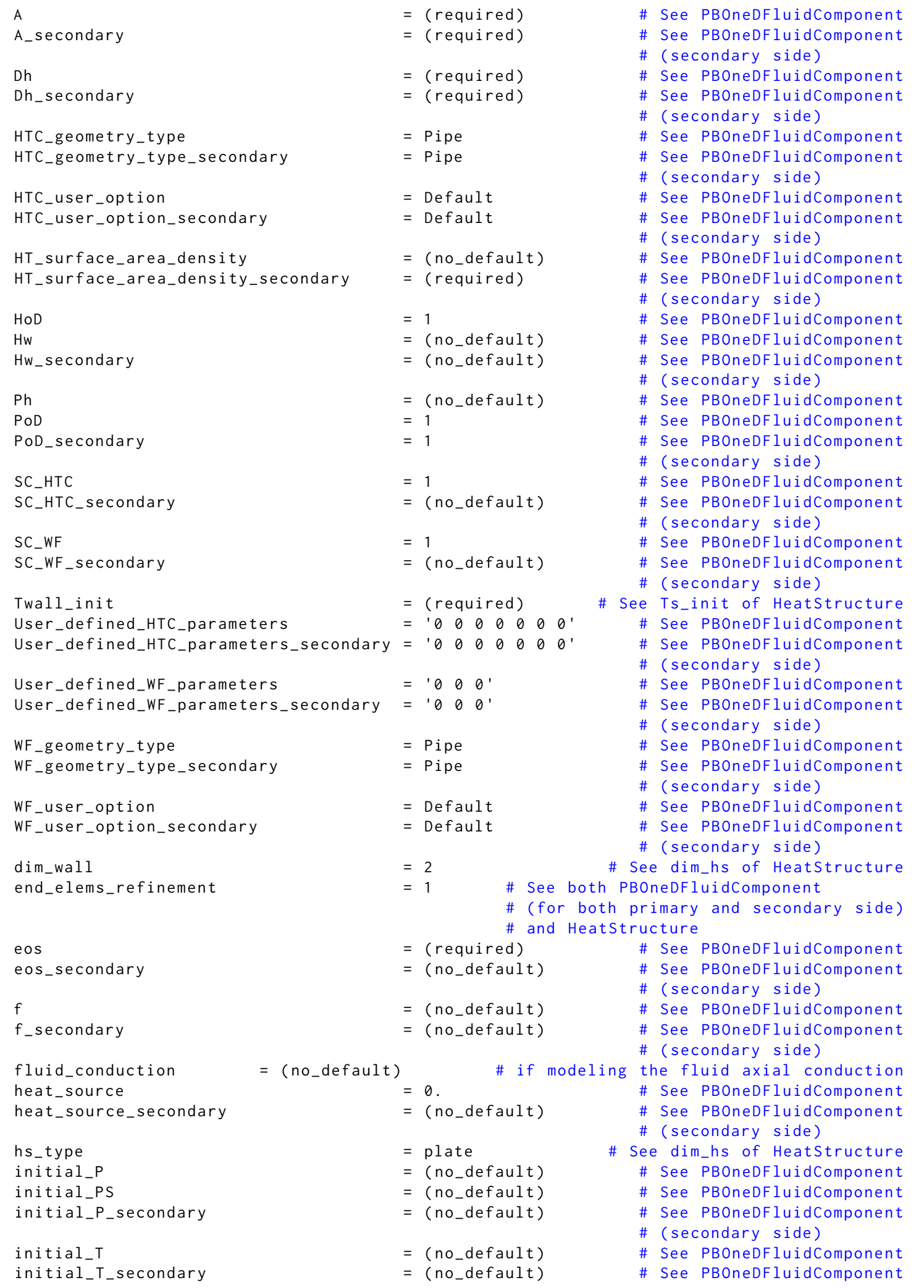




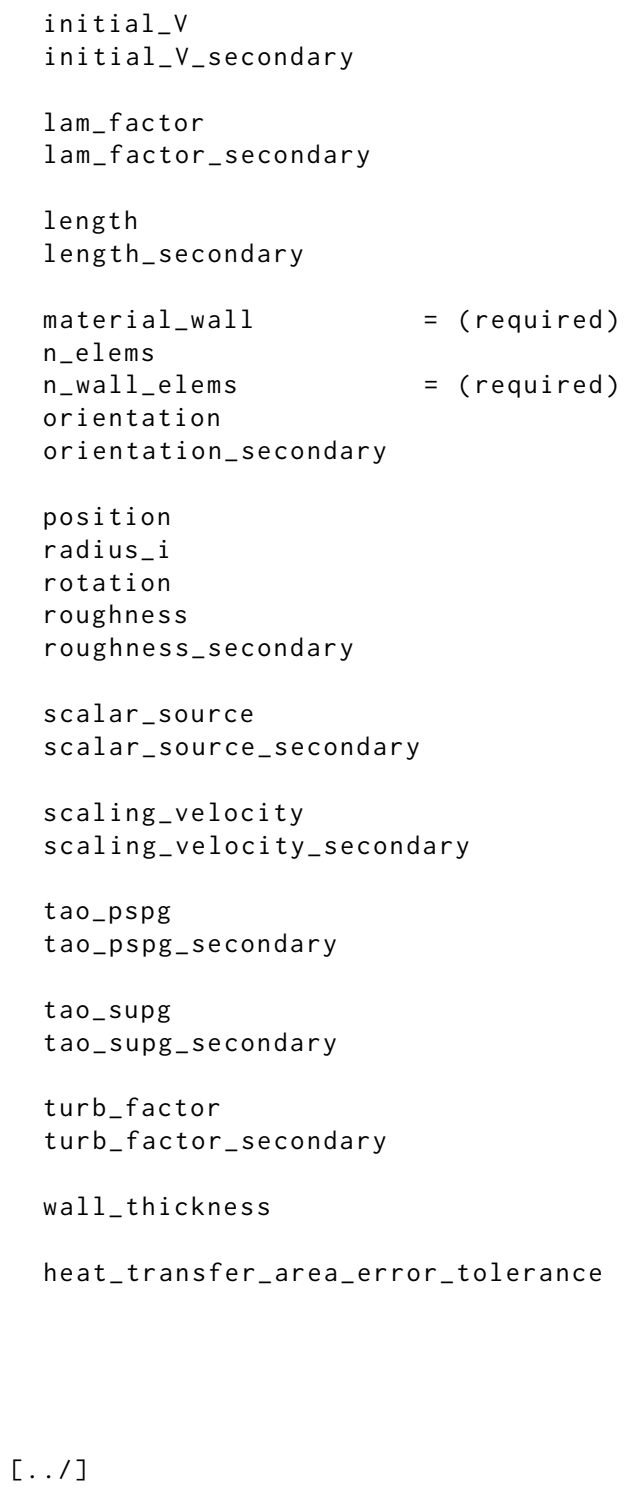

- HX_type

This input parameter specifies the type of heat exchanger, either "Concurrent" or "Countercurrent (default)". The two types of heat exchanger designs are shown in figure 4.11.

- heat_transfer_area_error_tolerance

This input parameter specifies an error tolerance, if the user-input parameters cause wall heat structure two-side surface areas inconsistence (see discussion below). The default value is $10^{-3}$.

Since the heat exchanger input parameters are organized in a way that the they are provided separately for three sub-components, i.e., the primary-side pipe, the secondary-side pipe, and the solid wall between them, user-input inconsistence could often raise, which could cause inconsistence 
in heat transfer areas between the two sides, and consequently introduces error in overall heat exchanger energy balance.

If the solid-wall heat structure is of "Plate" type, the heat transfer areas on both sides are the same, which requires,

$$
A_{p} a_{w, p} L_{p}=A_{s} a_{w, s} L_{s}
$$

and for "cylinder", the two-side areas follows,

$$
\frac{A_{p} a_{w, p} L_{p}}{A_{s} a_{w, s} L_{s}}=\frac{r_{p}}{r_{s}}
$$

in which, $A$ is flow area, $a_{w}$ is heat transfer area density, $L$ is pipe length, $r$ is the radius on wall surface; subscripts $p$ and $s$ denote the primary- and secondary-side, respectively.

The input heat transfer area error is then defined as,

$$
\varepsilon=\left|\frac{A_{p} a_{w, p} L_{p}}{A_{s} a_{w, s} L_{s}}-1\right|
$$

for plate type of wall heat structure, and

$$
\varepsilon=\left|\frac{A_{p} a_{w, p} L_{p}}{A_{s} a_{w, s} L_{s}}-\frac{r_{p}}{r_{s}}\right|
$$

for cylinder type of wall heat structure.

In PBHeatExchanger, it has

$$
r_{p}=r_{s}+\delta_{\text {wall }}
$$

where $\delta_{\text {wall }}$ is wall thickness (see wall_thickness), and $r_{s}$ is specified via radius_i. Thus, it requires that the primary-side is to be set at the shell-side of the heat exchanger.

- end_elems_refinement

This same input parameter is defined in both PBOneDFluidComponent and HeatStructure. If specified, the specified value will be passed to both PBOneDFluidComponent (the primary and secondary pipes) and HeatStructure to create finer meshes at the two ends of corresponding meshes.

This parameter is especially useful when the simulated fluid temperature experiences unphysical spatial oscillations near the inlet/outlet of PBHeatExchanger due to coarse mesh being used.

\subsubsection{PBTDJ}

PBTDJ is an inlet boundary component in which the flow velocity and temperature are provided by user-defined values or (time-dependent) functions. It provides boundary conditions to the connecting 1-D fluid components. Its input parameters are listed as follows:

$$
\begin{gathered}
\text { [. / PBTDJ] } \\
\text { S_bc } \\
\text { S_fn } \\
\text { T_bc } \\
\text { T_fn } \\
\text { eos }
\end{gathered}
$$

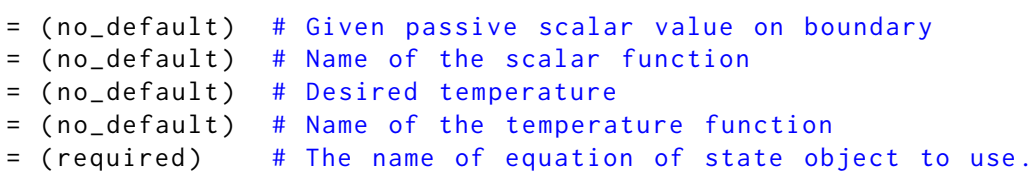




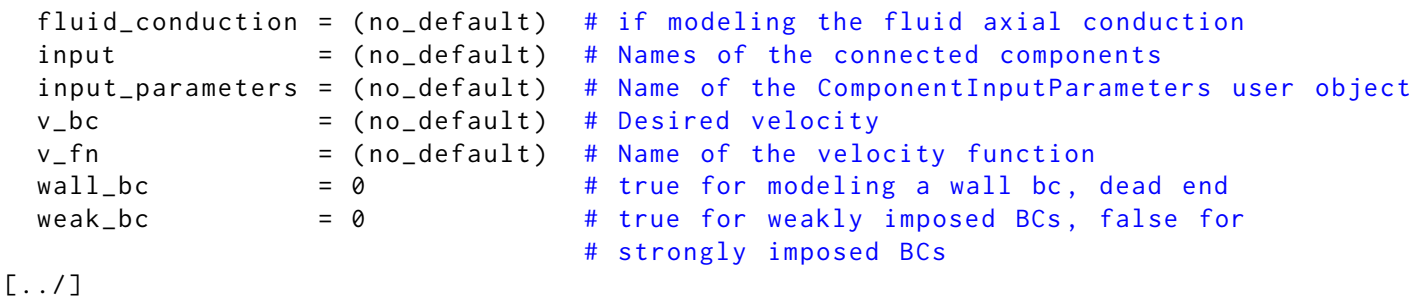

- eos (required)

The name of equation of state object to use for this PBTDJ component.

- T_bc

This input parameter specifies the inlet temperature, as a number, of this PBTDJ component.

- T_fn

This input parameter specifies the inlet temperature, as a function, of this PBTDJ component. If both T_bc and T_fn are specified, T_fn is used.

- v_bc

This input parameter specifies the inlet velocity, as a number, of this PBTDJ component.

- $v_{-} f n$

This input parameter specifies the inlet velocity, as a function, of this PBTDJ component. If both $v_{-} b c$ and $v_{-} f n$ are specified, $v_{-} f n$ is used.

- input

Name of the connected component and the connected end, (in) or (out), of the component, e.g., input $=$ 'pipe1(in)', and input $=$ 'pipe2(out)'.

- S_bc and S_fn

When passive scalars are present in the system, boundary conditions are also needed for them at this PBTDJ component. It first seeks a user input of S_fn, a list of function names to specify the boundary values of all scalars. If $S_{-} f n$ is not given, it then seeks $S_{-} b c$, a list of values to specify the boundary values of all scalars. If neither is specified, zero values are used as the boundary conditions for all scalar variables.

- fluid_conduction

This input parameter specifies if axial fluid conduction should be modeled. If not specified, it looks for the same input parameter in the global parameter inputs, see section 4.1.

- wall_bc

This input parameter specifies if a wall boundary condition, i.e., a dead end, should be modeled using this PBTDJ component. The default value is false.

- weak_bc

This input parameter specifies if a weakly imposed boundary condition should be used. The default value is false. 


\subsubsection{PBTDV}

PBTDV is a boundary component in which the pressure and temperature are provided by userdefined (time-dependent) functions. It provides boundary conditions to the connecting 1-D fluid components. Note if the flow is flowing into the PBTDV, the temperature boundary condition will not be used by the connecting fluid components. Its input parameters are listed as follows:

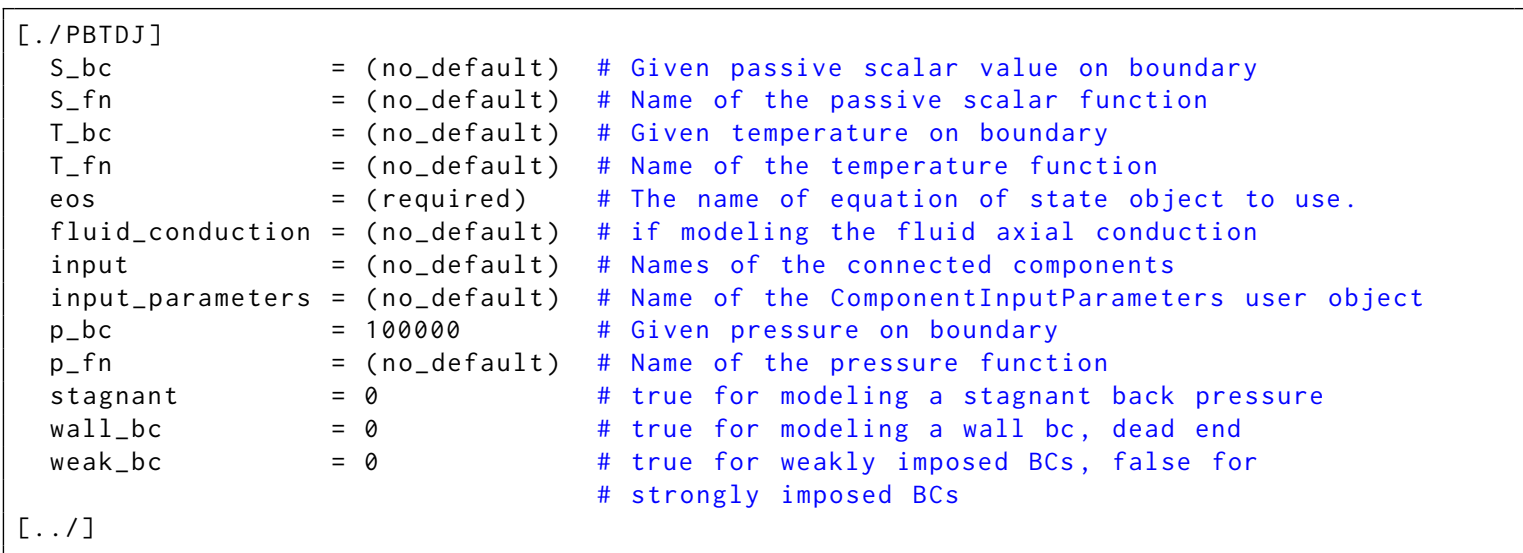

- eos (required)

The name of equation of state object to use for this PBTDJ component.

- T_bc

This input parameter specifies the inlet temperature, as a number, of this PBTDV component. It only matters when the flow direction is from PBTDV to its connected component.

- T_fn

This input parameter specifies the inlet temperature, as a function, of this PBTDV component. If both T_bc and T_fn are specified, T_fn is used.

- p_bc

This input parameter specifies the pressure, as a number, of this PBTDV component.

- $p_{-} f n$

This input parameter specifies the pressure, as a function, of this PBTDV component. If both $p_{-} b c$ and $p_{-} f n$ are specified, $p_{-} f n$ is used.

- stagnant

This input parameter specifies if stagnant pressure boundary condition should be used in this PBTDV component.

- input

Name of the connected component and the connected end, (in) or (out), of the component, e.g., input $=$ 'pipe1(in)', and input $=$ 'pipe2(out)'. 
- S_bc and S_fn

The same as discussed in PBTDJ, see section 4.3.16.

- fluid_conduction

This input parameter specifies if axial fluid conduction should be modeled. If not specified, it looks for the same input parameter in the global parameter inputs, see section 4.1.

- wall_bc

This input parameter specifies if a wall boundary condition, i.e., a dead end, should be modeled using this PBTDV component. The default value is false.

- weak_bc

This input parameter specifies if a weakly imposed boundary condition should be used. The default value is false.

\subsubsection{PressureOutlet}

PressureOutlet provides a subset of functionality of PBTDV, and will be removed in the future.

\subsubsection{CoupledTDV}

CoupledTDV is a special PBTDV component that is designed to facilitate the coupling between SAM and external CFD codes. Instead of user-specified values, in coupled code simulations, its boundary condition values are obtained from other external codes, and meanwhile, it also provides boundary conditions to these external codes. Compared with PBTDV, it does not require additional inputs, however, the code-to-code coupling is realized using CoupledCFDExecutioner.

\subsubsection{CoupledPPSTDJ}

CoupledPPSTDJ is a special PBTDJ component that is designed to facilitate MultiApp simulations. Instead of user-specified values, in MultiApp simulations, its boundary condition values are obtained from other MOOSE applications, and meanwhile, it also provides boundary conditions to other MOOSE applications. Inherited from PBTDJ, it requires two extra input parameters to facilitate MultiApp information passing between MOOSE applications.

- postprocessor_vbc and postprocessor_Tbc

This input parameter specifies a Postprocessor name, which will be used to specify the velocity (temperature) boundary condition values of this CoupledPPSTDJ component.

\subsubsection{CoupledPPSTDV}

Similar to CoupledPPSTDJ, CoupledPPSTDV is a special PBTDV component that is designed to facilitate MultiApp simulations. Instead of user-specified values, in MultiApp simulations, its boundary condition values are obtained from other MOOSE applications, and meanwhile, it also provides boundary conditions to other MOOSE applications. Inherited from PBTDV, it requires two extra input parameters to facilitate MultiApp information passing between MOOSE applications. 
- postprocessor_pbc and postprocessor_Tbc

This input parameter specifies a Postprocessor name, which will be used to specify the pressure (temperature) boundary condition values of this CoupledPPSTDJ component.

\subsubsection{PBSingleJunction}

PBSingleJunction is a special junction component, and it models a zero-volume flow joint where only two 1-D fluid components are connected. It thus does not need to model the mass, momentum, and energy conservations at the junction, but to assure that the two connecting nodes ( 1 and 2 ) have consistent boundary conditions.

Its input parameters are listed as follows:

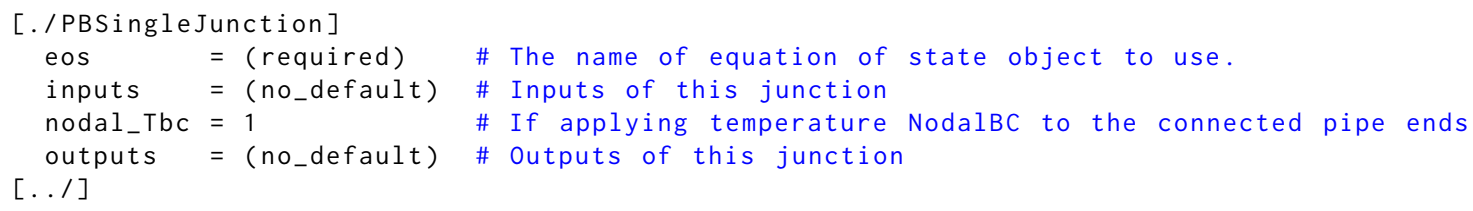

- eos (required)

The name of equation of state object to use.

- inputs and outputs

These input parameters specify the inputs and outputs connection of this PBSingleJunction component. The input syntax is, for example, inputs = 'pipe1(in)', and outputs = 'pipe2(out)'. There is only one inputs and one outputs allowed for this PBSingleJunction component.

- nodal_Tbc

This input parameter specifies if temperature NodalBC to be applied to the connected pipe ends. The default value is true.

\subsubsection{PBBranch}

PBBranch models a 0-D flow junction where multiple 1-D fluid components are connected. The component assumes no volume, and thus there is no thermal inertia.

Its input parameters are listed as follows:

\begin{tabular}{|c|c|c|}
\hline \multicolumn{3}{|l|}{$[. / \mathrm{PBBranch}]$} \\
\hline Area & $=($ required $)$ & \# Reference area of this branch \\
\hline K & $=($ required $)$ & \# Form loss coefficients \\
\hline K_B & $=($ no_default) & $\begin{array}{l}\text { \# coefficient B in calculating Reynolds number-dependent } \\
\text { \# form loss coefficients }\end{array}$ \\
\hline $\mathrm{K}_{-} \mathrm{B}_{-}$reverse & $=($no_default $)$ & $\begin{array}{l}\text { \# coefficient B in calculating Reynolds number-dependent } \\
\text { \# form loss coefficients in reverse direction }\end{array}$ \\
\hline $\mathrm{K}_{-} \mathrm{C}$ & $=($ no_default) & $\begin{array}{l}\text { \# coefficient C in calculating Reynolds number-dependent } \\
\text { \# form loss coefficients }\end{array}$ \\
\hline $\mathrm{K}_{-} \mathrm{C}_{-}$reverse & $=($no_default) & $\begin{array}{l}\text { \# coefficient } C \text { in calculating Reynolds number-dependent } \\
\text { \# form loss coefficients in reverse direction }\end{array}$ \\
\hline K_reverse & $=($ no_default) & \# Form loss coefficients in reverse direction \\
\hline eos & $=($ required $)$ & \# The name of equation of state object to use. \\
\hline initial_P & $=($ no_default $)$ & \# Initial pressure of this branch \\
\hline initial_T & $=($ no_default $)$ & \# Initial temperature of this branch \\
\hline
\end{tabular}




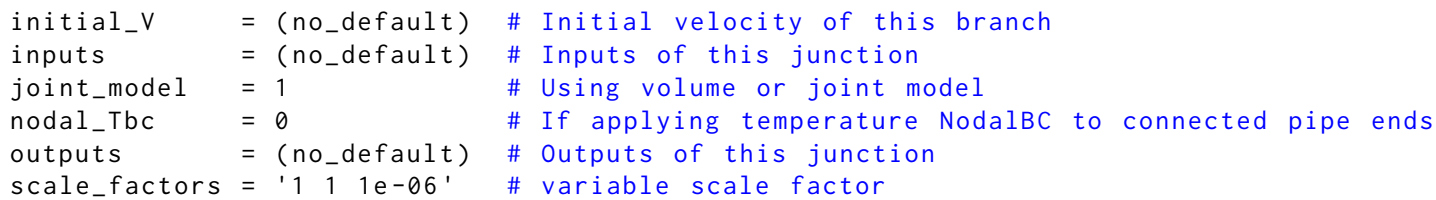

- eos (required)

The name of equation of state object to use.

- Area (required)

The reference area of this branch.

- inputs and outputs

These input parameters specify the inputs and outputs connection of this PBBranch component. The input syntax is, for example, inputs = 'pipe1(out) pipe2(out)', and outputs = 'pipe3(in)'.

- $\mathrm{K}$ (required)

This input parameter specifies a list of values for forward form loss coefficients at each connection of this PBBranch component. The total number of listed values has to be the same as the total number of connections. The forward direction is defined as if it flows from its connected 'inputs' pipes to this PBBranch component, or from this PBBranch component to its connected 'outputs' pipes. Otherwise, the flow is in reversed direction.

- K_reverse

Similar to K, this input parameter specifies the reverse flow form loss coefficients at each connection of this PBBranch component. It is not required, and if not specified, they assume the same values from the forward form loss coefficients. If a user input is given, the total number of listed values has to be the same as the total number of connections.

- K_B and K_C

These two input parameters supplement input parameter, $\mathrm{K}$, when forward form loss coefficients are Reynolds number-dependent,

$$
K_{\text {total }}=A+B \mathrm{Re}^{C}
$$

in which, $\mathrm{A}$ is the value from input parameter $\mathrm{K}, \mathrm{B}$ from $\mathrm{K}_{-} \mathrm{B}$, and $\mathrm{C}$ from $\mathrm{K}_{-} \mathrm{C}$. If specified, these two parameters have to be both given.

- K_B_reverse and K_C_reverse

These two input parameters are similar to K_B and K_C.

- nodal_Tbc

This input parameter specifies if temperature NodalBC to be applied to the connected pipe ends. The default value is true. 
- initial_P, initial_V, and initial_T

These input parameters specify the initial pressure (velocity, temperature) of this PBBranch component. If not specified, the component seeks the global initial values, see section 4.1.

- scale_factors

Similar to the global scaling factors, this input parameter specifies the local scaling factors for the three variables: pressure, velocity, and temperature. The default values are '1.0 1.0 1.0e-6'.

- joint_model (advanced)

This input parameter specifies if volume or joint model to be used in this PBBranch component. The default value is true.

\subsubsection{PBVolumeBranch}

PBVolumeBranch is a type of PBBranch while considering its volume effects, and thus, it accounts for the mass and energy balance between inlets and outlets, as well as its own volume. Inherited from PBBranch, PBVolumeBranch requires additional input parameters.

\begin{tabular}{|c|c|c|}
\hline [./PBVolumeBranc & $\mathrm{ch}]$ & \\
\hline Area & $=($ required $)$ & \# See PBBranch \\
\hline $\mathrm{K}$ & $=($ required $)$ & \# See PBBranch \\
\hline $\mathrm{K}_{-} \mathrm{B}$ & $=($ no_default $)$ & \# See PBBranch \\
\hline $\mathrm{K}_{-} \mathrm{B}_{-}$reverse & $=($no_default $)$ & \# See PBBranch \\
\hline $\mathrm{K}_{-} \mathrm{C}$ & $=($ no_default $)$ & \# See PBBranch \\
\hline $\mathrm{K}_{-} \mathrm{C}_{-}$reverse & $=($no_default $)$ & \# See PBBranch \\
\hline K_reverse & $=($ no_default $)$ & \# See PBBranch \\
\hline eos & $=($ required $)$ & \# See PBBranch \\
\hline initial_P & $=($ no_default $)$ & \# See PBBranch \\
\hline initial_T & $=($ no_default $)$ & \# See PBBranch \\
\hline initial_V & $=($ no_default $)$ & \# See PBBranch \\
\hline inputs & $=($ no_default $)$ & \# See PBBranch \\
\hline joint_model & $=1$ & \# See PBBranch \\
\hline nodal_Tbc & $=0$ & \# See PBBranch \\
\hline outputs & $=($ no_default $)$ & \# See PBBranch \\
\hline scale_factors & $=' 111 \mathrm{e}-06^{\prime}$ & \# See PBBranch \\
\hline Steady & $=0$ & \# for steady state initialization \\
\hline center & $=($ required $)$ & \# geometric center of the volume \\
\hline display_pps & $=0$ & \# display post processors \\
\hline height & $=($ no_default $)$ & \# Height of the component \\
\hline orientation & $=10011$ & \# Orientation vector of the component \\
\hline position & $=10001$ & \# Origin (start) of the component \\
\hline rotation & $=0$ & \# Rotation of the component (in degrees) \\
\hline volume & $=($ required $)$ & \# Volume of the component \\
\hline width & $=($ no_default $)$ & \# Width of the component \\
\hline$[\ldots /]$ & & \\
\hline
\end{tabular}

- center (required)

The geometric center of the volume, which is important to compute pressure jump between this PBVolumeBranch and its connected pipes due to gravity head. It also overrides the values given in position (if ever specified).

- volume (required)

The Volume of the component. 
- display_pps

PBVolumeBranch adds several Postprocessors to monitor its field variables (pressure, temperature, density, and velocity). If specified true, these Postprocessors values will be displayed. The default value is false.

- Steady

This input parameter specifies if the initial values are to be used for steady state initialization. The default value is false.

- width and height

For display purpose, these parameters specify the width and height of this PBVolumeBranch component. For width, if not specified, it is computed as the pipe diameter as if the PBVolumeBranch was a round pipe section, i.e., width $=2 \sqrt{A / \pi}$, in which $\mathrm{A}$ is the reference area. For height, if not specified, it is computed as volume divided by the reference area.

- orientation, position, and rotation

See PBOneDFluidComponent, section 4.3.1. In this component, they are used to generate mesh for display purpose.

\subsubsection{PBLiquidVolume}

PBLiquidVolume is a special PBVolumeBranch, in which the liquid volume can change due to in and out flows, and the liquid level is tracked during the transient. The reference gas phase pressure in the PBLiquidVolume is either an ambient pressure, figure 4.12 (a), or comes from an external component, CoverGas, figure 4.12 (b). For CoverGas component, see section 4.3.26.

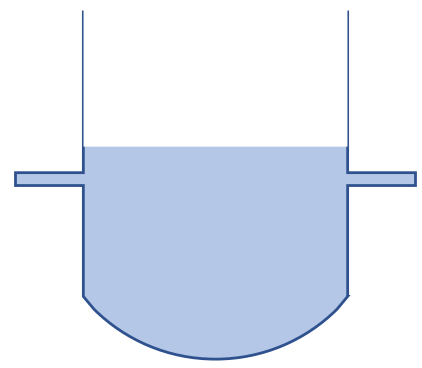

(a)

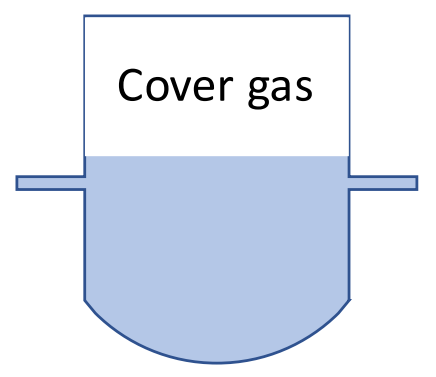

(b)

Figure 4.12: The PBLiquidVolume concept used in SAM. (a) PBLiquidVolume with ambient pressure as its reference pressure; (b) PBLiquidVolume with an external CoverGas to specify its reference pressure.

Additional to what are needed in PBVolumeBranch (see section 4.3.24), PBLiquidVolume requires several additional input parameters for the initial liquid level and the external CoverGas component, or an ambient pressure.

- initial_level (required)

The initial liquid level in this PBLiquidVolume component. 
- covergas_component

The name of the external CoverGas component, the pressure of which will be used as the reference pressure in this PBLiquidVolume component. If not specified, PBLiquidVolume takes an ambient pressure as the reference pressure. To compute the liquid phase pressure, a hydrostatic pressure head will be added to this reference pressure. The hydrostatic pressure head is calculated as $\rho g L$, with $\rho$ the liquid density, $g=9.81$ the gravity constant, and $L$ the liquid level.

- ambient_pressure

If a CoverGas component is not given to provide the reference pressure, an ambient pressure is then needed as the reference pressure. The default value is 1 bar $\left(10^{5} \mathrm{~Pa}\right)$.

\subsubsection{CoverGas}

CoverGas component is always used together with PBLiquidVolume component, see figure 4.12 (b). It models a 0-D gas volume that is connected to one or multiple liquid volumes. The gas volume is modeled as an ideal gas, and the heat transfer between the cover gas and the liquid volumes is neglected. Its volume change is decided by the volume changes of all connecting liquid volumes.

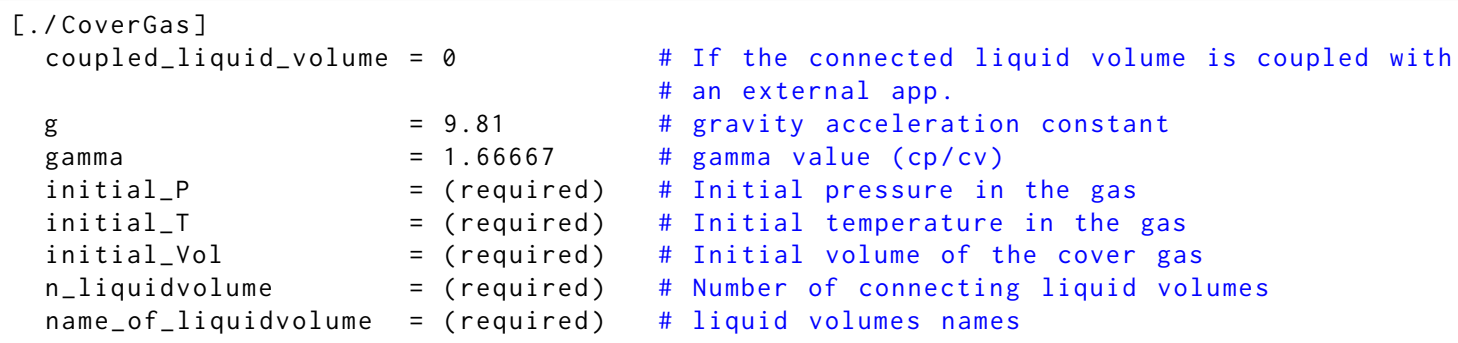

- initial_P (required) and initial_T (required)

The initial pressure and temperature of the gas phase.

- initial_Vol (required)

The initial volume of the gas phase.

- n_liquidvolume (required)

Number of connected liquid volumes.

- name_of_liquidvolume (required)

A list of names of connected PBLiquidVolume components. The total number of these connected PBLiquidVolume components has to be the same as n_liquidvolume. The changes of the liquid volume in these connected PBLiquidVolume will be used to determine the gas phase volume in this CoverGas component, consequently, its pressure.

- gamma

Gamma value to be used in the ideal gas equation of state that computes the gas phase pressure as its volume changes. The default value is 1.66667 . 
- coupled_liquid_volume

This input parameter specifies if the connected liquid volume is coupled with an external app. The default value is false.

- $\mathrm{g}$

Gravity acceleration constant. The pressure difference between the gas phase and its connected PBLiquidVolume liquid phase is $\rho g L$, with $\rho$ the liquid density, $g$ the gravity acceleration constant, and $L$ the liquid level in PBLiquidVolume component.

\subsubsection{PBPump}

PBPump is another special junction component, and it simulates a pump, in which the pump head can be dependent on a pre-defined function, or can be automatically adjusted to match userspecified mass flow rate. It inherits from the PBBranch component, and therefore assumes the volume of the pump is neglectable. More complex pump models will be developed in future SAM enhancements. Pumping power can be modeled and considered in the energy conservation of the junction.

Its input parameters are listed as follows:

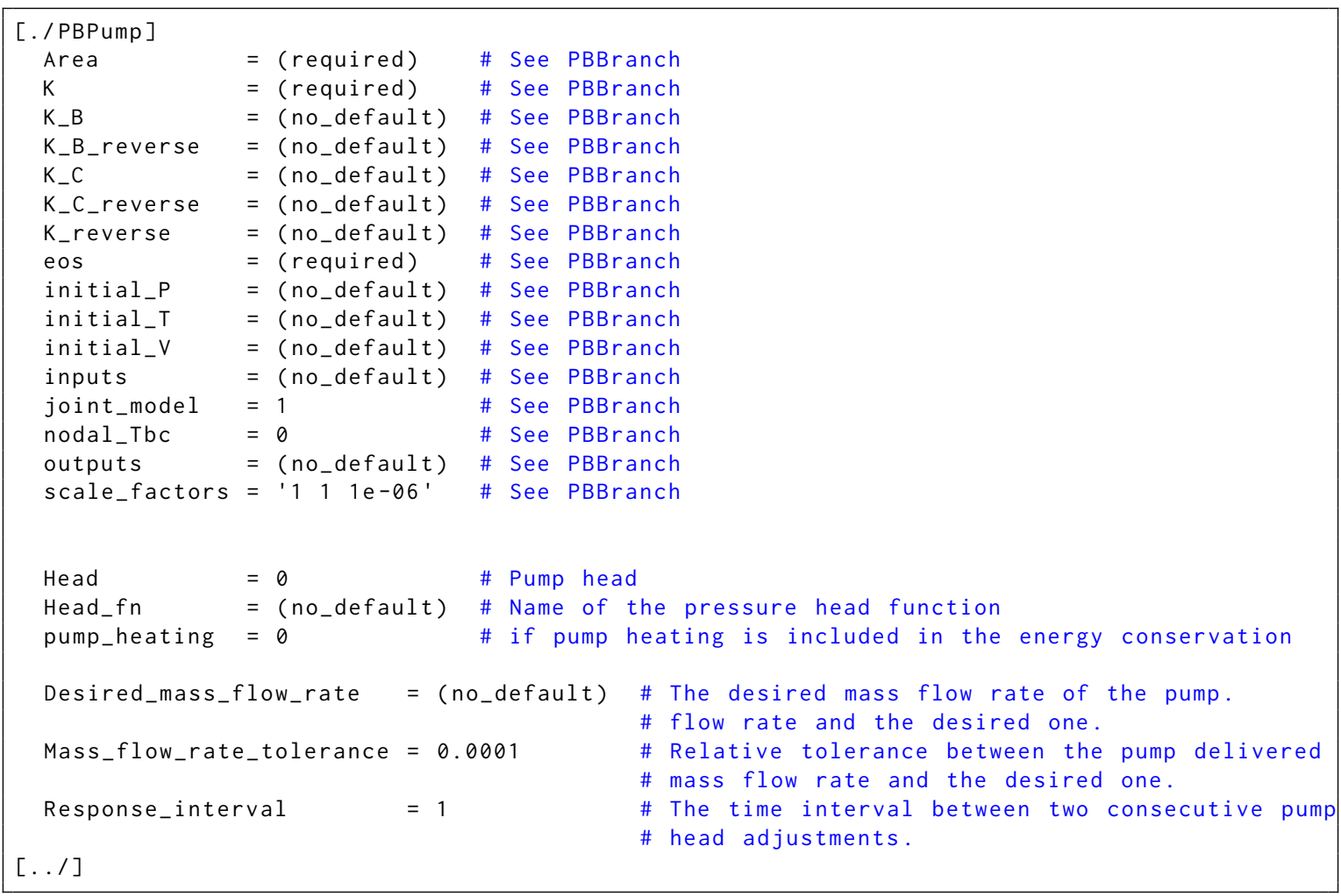

- Head

This input parameter specifies the pump head value, in [Pa]. The default value is 0 .

- Head_fn

This input parameter specifies the function name to compute pump head value. 
- pump_heating

This input parameter specifies if pump heating effect should be considered in energy balance. The default is false.

- Desired_mass_flow_rate

This input parameter accepts a user-specified mass flow rate, such that the pump will automatically adjust the pump head to match this value. If specified, the pump head specified in the Head input parameter is used as the initial guessing value to start with the automatical adjustment.

- Mass_flow_rate_tolerance

When a user-specified mass flow rate is given, this input parameter the absolute relative tolerance between the real pump mass flow rate compared with the user-specified one. If within this tolerance, the pump stops to adjust its pump head as it is deemed that the desired mass flow rate already achieved. The default value is $10^{-4}$.

- Response_interval

When a user-specified mass flow rate is given, this input parameter specifies the how fast, i.e., the time internal between two consecutive pump head adjustments. The default value is 1 second.

\subsubsection{StagnantVolume}

StagnantVolume models a stagnant liquid volume, which has no connections to 1-D fluid components but is allowed to connect to a 0-D volume or 1-D or 2-D heat structures for heat transfer. It is assumed that there is no net mass transfer between StagnantVolume and the connecting 0-D volumes. Its input parameters are listed as follows:

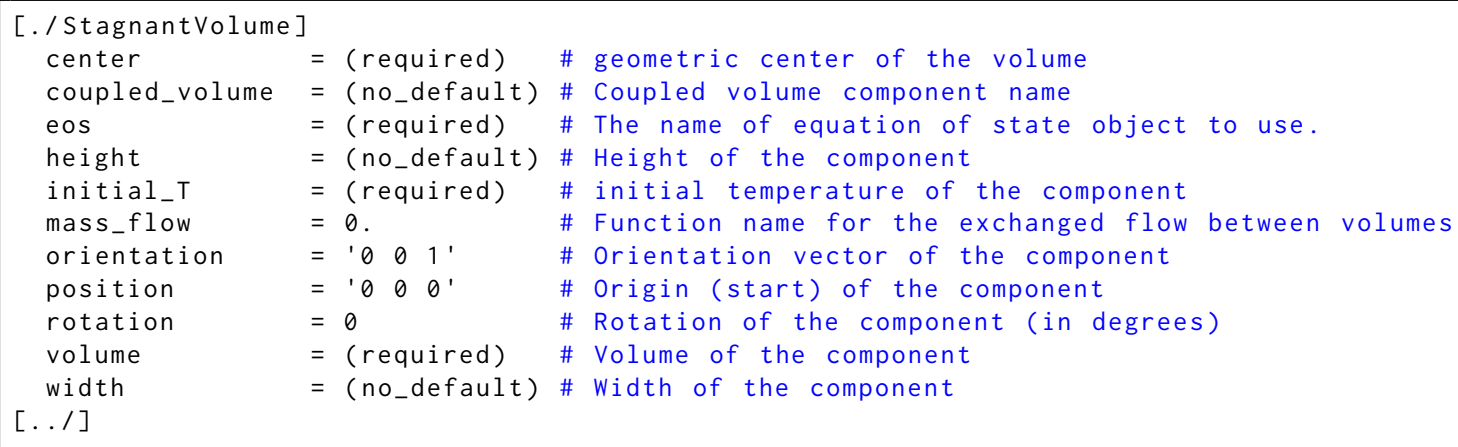

- center (required)

The geometric center of the volume. It overrides the values given in position (if ever specified), and is used to generate mesh for display purpose.

- eos (required)

The name of equation of state object to use.

- initial_T (required)

The initial fluid temperature of the component. 
- height and width

These input parameters specify the height and width of the component, both of which are to be used to generate mesh for display purpose. The default value for both parameters are $1 \mathrm{~m}$.

- coupled_volume

The name of coupled volume, e.g., another StagnantVolume, which this StagnantVolume component exchanges energy with. The energy exchange between these two volumes is computed as $\dot{m} \overline{c_{p}} \Delta T$, in which $\dot{m}$ is the mixing mass flow rate (see mass_flow), $\overline{c_{p}}$ is the specific heat computed at the average temperature between the two mixing volume, $\Delta T$ the temperature difference between the two mixing volume.

- mass_flow

If a coupled volume is specified, this input parameter specifies the mixing mass flow rate between the two mixing volume. It can be either a number or a function name.

- orientation, position, and rotation

Parameters not used.

\subsubsection{LiquidTank}

The LiquidTank component of SAM simulates a PBVolumeBranch (or PBLiquidVolume) and the heat structure (modeled as PBCoupledHeatStructure) attached to it in order to capture this additional thermal inertia. The input parameters of the LiquidTank component requires those to describe the PBVolumeBranch (or PBLiquidVolume) and those to describe the PBCoupledHeatStructure attached to it.

The LiquidTank component automatically create a PBLiquidVolume component, if a CoverGas component is connected to determine its gas phase pressure; otherwise, a PBVolumeBranch component is create. It assumes that the PBVolumeBranch (or PBLiquidVolume) is connected to the left-side surface of PBCoupledHeatStructure, and additional boundary condition input parameters are required for the right-side surface of PBCoupledHeatStructure.

The list of input parameters are given in the following list. Part of them are required to describe the PBVolumeBranch (or PBLiquidVolume) component, which could be referred to section 4.3.24 (or section 4.3.25); and part of them are required to describe the PBCoupledHeatStructure component, which could be referred to section 4.3.12.

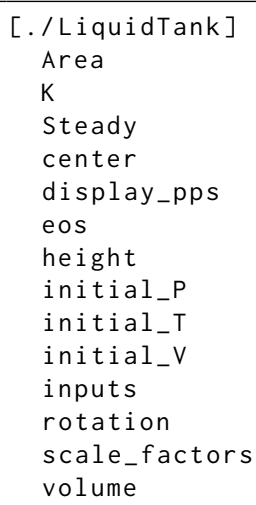

$=($ required $)$

$=$ (required)

$=0$

$=$ (required)

$=0$

$=$ (required)

$=$ (no_default)

$=$ (no_default)

$=$ (no_default )

$=$ (no_default)

$=$ (no_default)

$=0$

$=$ ' $^{1} 11 \quad 1 \mathrm{e}-06^{\prime}$

$=$ (required)

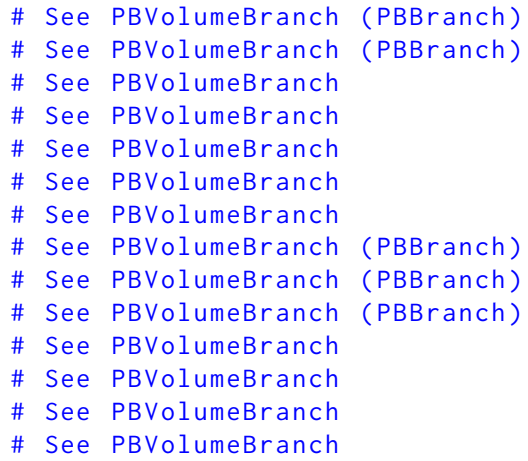




\begin{tabular}{|c|c|c|}
\hline width & $=($ no_default $)$ & \# See PBVolumeBranch \\
\hline nodal_Tbc & $=1$ & \# See PBVolumeBranch (PBBranch) \\
\hline orientation & $=10011$ & \# See PBVolumeBranch \\
\hline outputs & $=($ no_default) & \# See PBVolumeBranch \\
\hline position & $=1000^{\prime}$ & \# See PBVolumeBranch \\
\hline HS_BC_type_right & $=($ required $)$ & \# See HS_BC_type of PBCoupledHeatStructure \\
\hline HT_surface_area_density_right & $=($ no_default) & \# See PBCoupledHeatStructure \\
\hline & $=($ no_default $)$ & \# See Hw_left of PBCoupledHeatStructure \\
\hline $\mathrm{Hw}_{-} \mathrm{right}$ & $=($ no_default $)$ & \# See PBCoupledHeatStructure \\
\hline T_amb_right & $=300$ & \# See PBCoupledHeatStructure \\
\hline T_bc_right & $=600$ & \# See PBCoupledHeatStructure \\
\hline Ts_init & $=($ no_default $)$ & \# See PBCoupledHeatStructure \\
\hline dim_hs & $=2$ & \# See PBCoupledHeatStructure \\
\hline elem_number_axial & $=1$ & \# See PBCoupledHeatStructure \\
\hline elem_number_radial & $=($ required $)$ & \# See PBCoupledHeatStructure \\
\hline eos_right & $=($ no_default $)$ & \# See PBCoupledHeatStructure \\
\hline heat_source_solid & $=0$ & \# See PBCoupledHeatStructure \\
\hline heat_transfer_area & $=($ no_default $)$ & $\begin{array}{l}\text { \# See heat_transfer_area_left } \\
\text { \# of PBCoupledHeatStructure }\end{array}$ \\
\hline heat_transfer_area_right & $=($ no_default) & \# See PBCoupledHeatStructure \\
\hline hs_names & $=($ no_default) & \# See PBCoupledHeatStructure \\
\hline hs_type & $=$ cylinder & \# See PBCoupledHeatStructure \\
\hline length & $=($ required $)$ & \# See PBCoupledHeatStructure \\
\hline material_hs & $=($ required $)$ & \# See PBCoupledHeatStructure \\
\hline name_comp_right & $=($ no_default $)$ & \# See PBCoupledHeatStructure \\
\hline qs_right & $=($ no_default $)$ & \# See PBCoupledHeatStructure \\
\hline radius_i & $=($ no_default $)$ & \# See PBCoupledHeatStructure \\
\hline width_of_hs & $=($ required $)$ & \# See PBCoupledHeatStructure \\
\hline initial_level & $=($ no_default $)$ & \# See PBLiquidVolume \\
\hline covergas_component & $=($ no_default $)$ & \# See PBLiquidVolume \\
\hline $\begin{array}{l}\text { mesh_disp_gap } \\
. /]\end{array}$ & $=0.1$ & \# Axial offset for mesh generation \\
\hline
\end{tabular}

Some details of the input parameters as discussed as follows.

- mesh_disp_gap

This input parameter specifies mesh offset in the y-direction, with respect to the fluid component mesh, when creating heat structure meshes. The default value for this parameter is $0.1[\mathrm{~m}]$. If 'cylinder' is specified for hs_type, this mesh offset value will be overridden by half of radius_i value.

\subsubsection{ReactorCore}

The ReactorCore component describes a pseudo three-dimensional reactor core by connecting bypass channels to their neighboring core channels (with duct walls). Its input parameters are listed below.

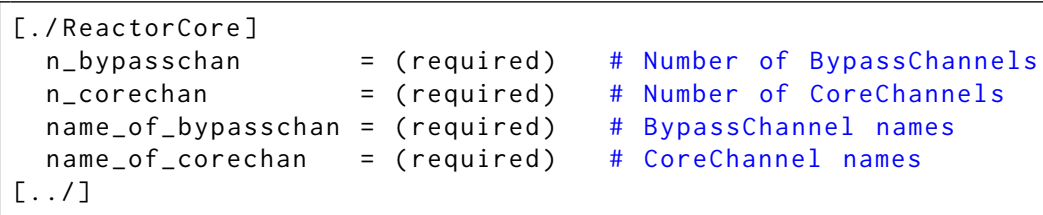

- n_bypasschan (required) 
The total number of bypass channels in this ReactorCore component.

- n_corechan (required)

The total number of core channels in this ReactorCore component.

- name_of_bypasschan (required)

The names of all bypass channels.

- name_of_corechan (required)

The names of all core channels.

\subsubsection{SurfaceCoupling}

The SurfaceCoupling component models the heat transfer between two solid surfaces, suitable for radiation heat transfer or gap heat transfer between them.

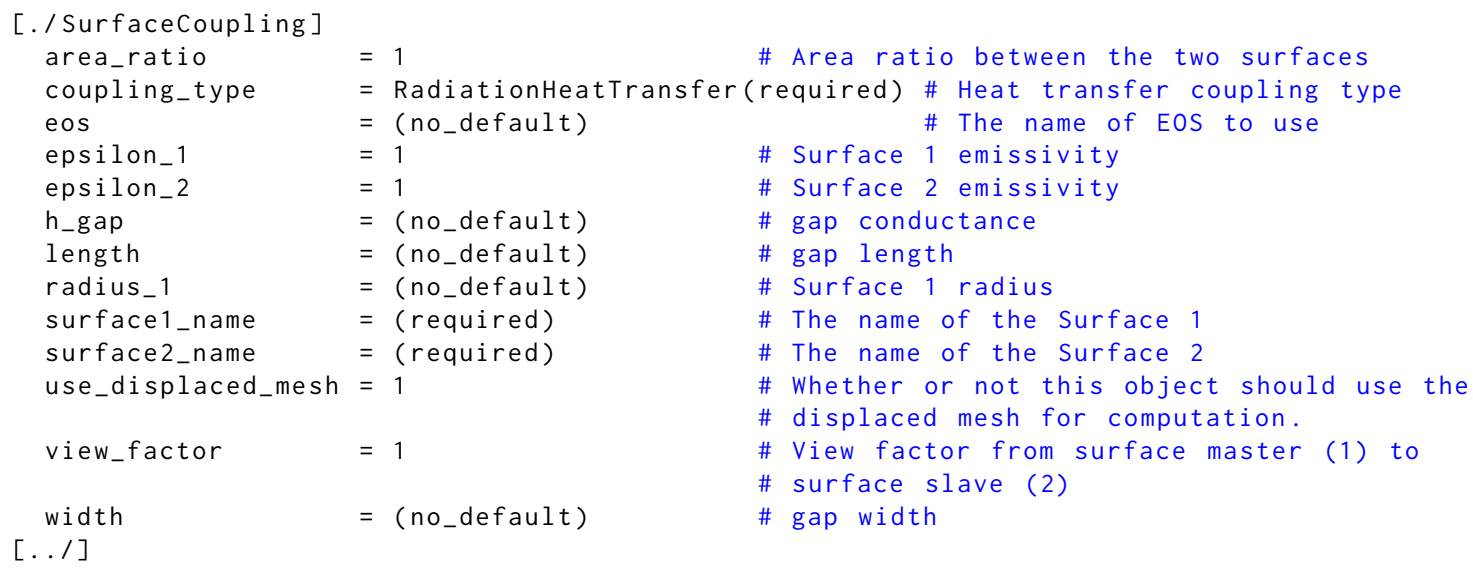

- surface1_name (required) and surface2_name (required)

The name of surface 1 (2) that participates in the radiation or gap heat transfer.

- coupling_type (required)

The heat transfer mechanism type of the heat transfer, either 'RadiationHeatTransfer' for radiation heat transfer, or 'GapHeatTransfer' for gap heat transfer. The default value is 'RadiationHeatTransfer'.

- area_ratio

The ratio of surface 1 area to surface 2 area, which is only required for radiation heat transfer to compute the heat flux between the two surfaces. If not specified, the default value is 1 .

- radius_1

The radius of surface 1 , if surface 1 is of cylindrical type. 
- epsilon_1 and epsilon_2

The emissivity of surface 1 (2), only required for radiation heat transfer mechanism. Both parameters have the same default value 1 .

- view_factor

This parameter defines the view factor between surfaces 1 and 2, only required for radiation heat transfer mechanism. The default value is 1 .

- h_gap

For gap heat transfer mechanism, if user-specified value is desired for the gap heat transfer coefficient, this input parameter specifies such a value. If not specified, SAM will compute the gap heat transfer coefficient from other input parameters.

- eos

The equation of state that will be used to compute gap heat transfer coefficient, only required when the heat transfer mechanism is gap heat transfer, and when user-specified heat transfer coefficient is not given.

- width and length

Gap width (length), only required when the heat transfer mechanism is gap heat transfer, and when user-specified heat transfer coefficient is not given.

- use_displaced_mesh

This parameter specifies that if displaced mesh to be used. The default value is true, and it is safe to use this default value.

\subsubsection{ReactorPower}

ReactorPower is a non-geometric component for describing the total reactor power, which can be dependent on either user-defined functions (such as describing the decay heat curve), or computed externally from SAM's PointKinetics component (see section 4.3.33). The total reactor power variable is used in core components such as PBCoreChannel and PBBypassChannel.

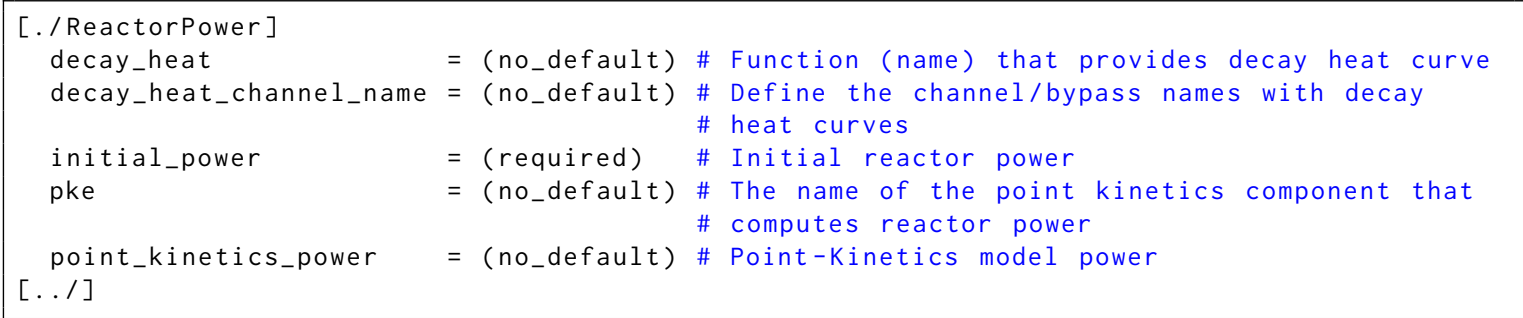

- initial_power (required)

This input parameter specifies the initial power of the reactor.

- decay_heat

If a decay heat curve is to be used to compute the reactor power, this input parameter specifies the decay heat curve function name. 
- pke

If an external PointKinetics component is to be used to compute the reactor power, this input parameter specifies the name of the PointKinetics component.

- point_kinetics_power

If an external PointKinetics component is to be used to compute the reactor power, this input parameter specifies the PointKinetics power.

- decay_heat_channel_name

This input parameter specifies the core channel and/or bypass channel names with decay heat curves.

\subsubsection{PointKinetics}

The PointKinetics component is the build-in point kinetics model of SAM, which models the transient behaviors of reactor fission power, delayed-neutron precursors, as well as reactivity feedback from other components, e.g., core channels. In case of modeling molten-salt reactors, where drifting delayed neutron precursors effect cannot be ignored, PointKinetics component also take account into the net flow in (out) effect as an additional source (sink) term. The net flow in (out) effect is captured in a coupled PBMoltenSaltChannel component.

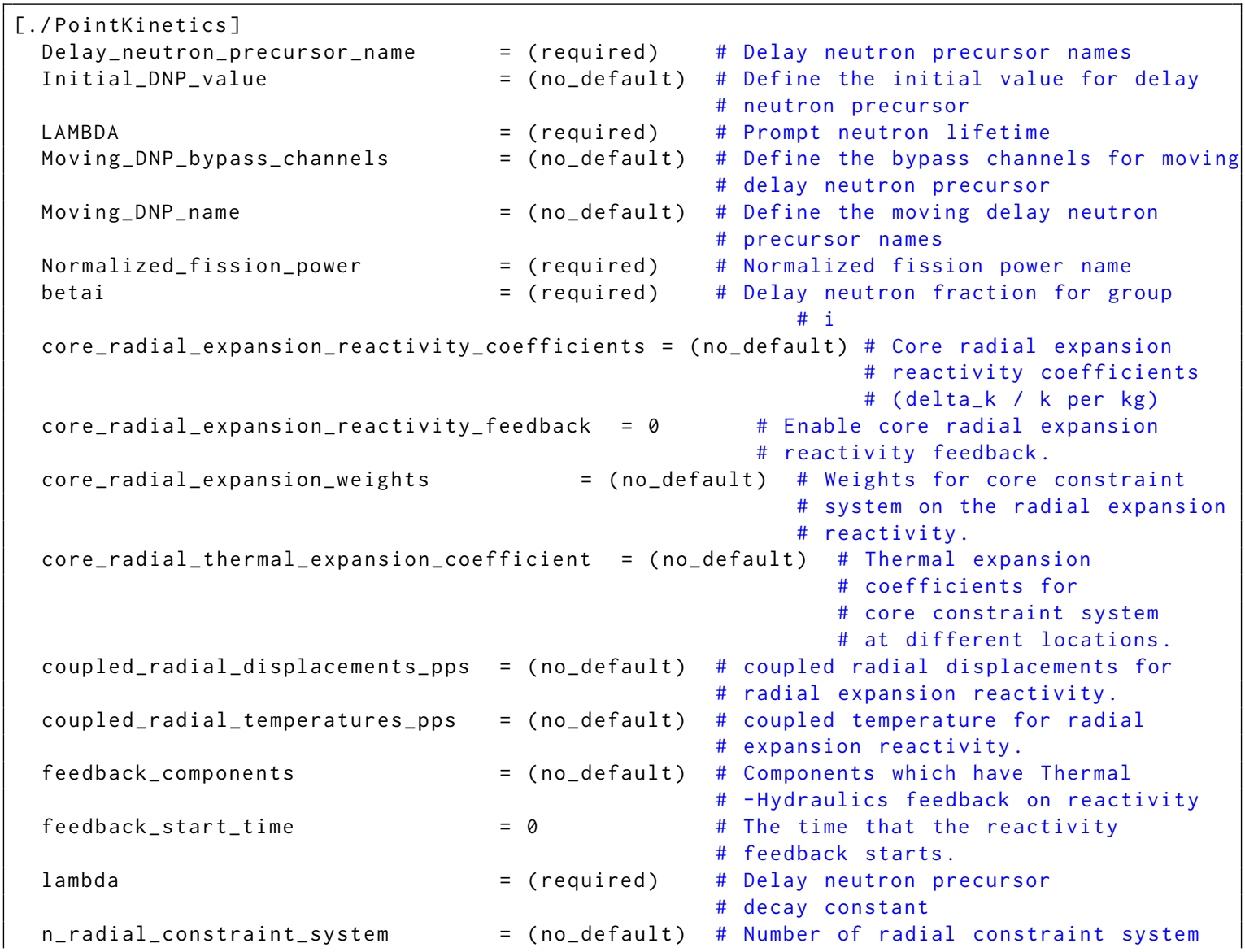




\begin{tabular}{|c|c|c|}
\hline & & $\begin{array}{l}\text { \# for core radial expansion reactivity } \\
\text { \# feedback. }\end{array}$ \\
\hline rho_fn_name & $=($ no_default $)$ & $\begin{array}{l}\text { \# External reactivity (delta } \mathrm{k} \text { per } \\
\text { \# k) }\end{array}$ \\
\hline use_external_radial_displacement & $=0$ & $\begin{array}{l}\text { \# Enable coupled radial displacement } \\
\text { \# from external thermo-mechanical } \\
\text { \# module. }\end{array}$ \\
\hline$\ldots /]$ & & \\
\hline
\end{tabular}

- Delay_neutron_precursor_name (required)

This input parameter specifies a list of names for delayed neutron precursors.

- Initial_DNP_value

This input parameter specifies a list of initial values for delayed neutron precursor populations. The total number of list values have to be the same as the total number of delayed neutron precursor names. However, if not specified, delayed neutron precursor populations are initialized as:

$$
C_{i, \text { initial }}=\frac{\beta_{i}}{\Lambda \lambda_{i}}
$$

in which, $\beta_{i}$ is the delayed neutron precursor fraction for group i (see betai), $\Lambda$ the prompt neutron lifetime (see LAMBDA), $\lambda_{i}$ the delayed neutron precursor decay constant for group i (see lambda).

- LAMBDA (required)

This input parameter specifies the prompt neutron lifetime.

- lambda (required)

This input parameter specifies a list of decay constants for delayed neutron precursors. The total number of list values have to be the same as the total number of delayed neutron precursor names.

- betai (required)

This input parameter specifies a list of delayed neutron precursor fractions. The total number of list values have to be the same as the total number of delayed neutron precursor names.

- Normalized_fission_power(required)

This input parameter specifies the name of the normalized fission power variable.

- rho_fn_name

This input parameter specifies the function name to introduce an external reactivity, additional to those from reactor feedbacks, to the PointKinetics model. If not specified, this external reactivity is 0 .

- feedback_components

Besides external reactivity function, the other main reactivity feedback mechanism is thermalhydraulics feedback from reactor core channel components. This input parameter specifies a list of components from which reactivity feedback will be computed. 
- feedback_start_time

The time that reactor core channel components start to compute reactivity feedback. The default value is 0 second.

- core_radial_expansion_reactivity_feedback

This input parameter specifies if core radial expansion reactivity feedback should be modeled. The default value is false. If modeled, the total reactivity feedback due to core radial expansion is computed as,

$$
\Delta R_{\text {radialexpansion }}=\sum_{i=1}^{N}\left(\frac{\Delta L}{L}\right)_{i} w_{i} \rho_{i}
$$

in which,

$N$ is the the total number of radial sections to compute reactivity feedback;

see n_radial_constraint_system.

$(\Delta L / L)_{i}$ is the radial core displacement value in the $\mathrm{i}$-th radial section;

$w_{i}$ is the weight value of the $\mathrm{i}$-th radial section; see core_radial_expansion_weights.

$\rho_{i}$ is core radial expansion coefficient at the $\mathrm{i}$-th radial section;

see core_radial_expansion_reactivity_coefficients.

Currently, SAM provides two ways to model core radial expansion, i.e., a simple built-in function or coupled from external simulations. The simpler one is a built-in function, which computes the i-th radial expansion as,

$$
\left(\frac{\Delta L}{L}\right)_{i}=\alpha_{i}\left(T_{i}-T_{0, i}\right)
$$

in which,

$\alpha_{i}$ is the thermal expansion coefficient of the constraint structure at the $\mathrm{i}$-th radial section, see thermal_expansion_coefficient.

$T_{i}$ is the core temperatures in the $\mathrm{i}$-th radial section;

see coupled_radial_temperatures_pps.

$T_{0, i}$ is the initial, i.e., when reactor core channel components start to compute reactivity feedback, core temperatures in the $\mathrm{i}$-th radial section.

The radial core displacement can also be modeled from an external code, and then coupled with SAM to compute the reactivity feedback value (use_external_radial_displacement $=$ true).

- use_external_radial_displacement

This input parameter specifies if core radial displacement should be modeled from an external simulation and their values are provided as coupled values. The default value is false. If specified true, externally computed core radial expansion displacement is expected from user input, see coupled_radial_displacements_pps. 
- n_radial_constraint_system

If core radial expansion reactivity feedback is modeled, this input parameter specifies the number of radial constraint system, i.e., number of core radial sections, for core radial expansion reactivity feedback.

- core_radial_expansion_reactivity_coefficients

This input parameter specifies a list of core radial expansion reactivity feedback coefficient values. The total number of this list of values has to be the same as the number of core radial sections (see n_radial_constraint_system).

- core_radial_expansion_weights

This input parameter specifies a list of values for core radial expansion reactivity feedback weights. The total number of this list of values has to be the same as the number of core radial sections (see n_radial_constraint_system).

- thermal_expansion_coefficient

If radial expansion is modeled used SAM's built-in function, this input parameter specifies a list of thermal expansion coefficients to compute radial core displacement values. The total number of this list of values has to be the same as the number of core radial sections (see n_radial_constraint_system).

- coupled_radial_temperatures_pps

If radial expansion is modeled used SAM's built-in function, this input parameter specifies a list of Postprocessor names that compute core temperatures in each radial section. The total number of this list of names has to be the same as the number of core radial sections (see n_radial_constraint_system).

- coupled_radial_displacements_pps

If core radial displacement should be modeled from an external simulation and their values are provided as coupled values, this input parameter specifies a list of Postprocessor names that compute core radial displacement at each radial section. The total number of this list of names has to be the same as the number of core radial sections (see n_radial_constraint_system).

- Moving_DNP_bypass_channels

If drifting delayed neutron precursors effect should be considered, this input parameter specifies the PBMoltenSaltChannel component name, from which the net flow in (out) of drifting delayed neutron precursors are computed.

- Moving_DNP_name

If drifting delayed neutron precursors effect should be considered, this input parameter specifies the names of these delayed neutron precursors. 


\subsubsection{ReferenceBoundary}

ReferenceBoundary component provides a fixed value boundary condition to a one-dimensional fluid type of component. This boundary condition can be applied to normal flow parameters, such as pressure, velocity, and temperature, as well as scalar variables.

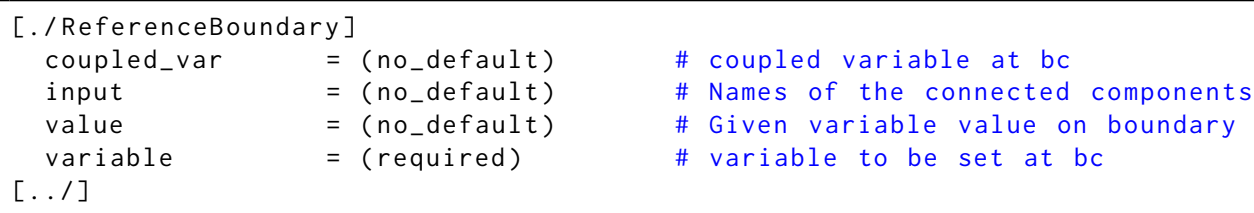

- input

This input parameter specifies where this boundary condition should be applied, e.g., input = 'pipe-1(in)'.

- variable (required)

This input parameter specifies which variable this boundary condition should be applied, e.g., variable = pressure. In principle, this can be any field variable, but pressure is commonly used to setup the system reference pressure.

- value

This input parameter specifies the value to be applied to the variable in this boundary condition.

- coupled_var

This input parameter specifies a coupled variable, whose value is to be applied to the variable in this boundary condition.

\subsubsection{PipeChain}

PipeChain is a non-geometric component for sequentially connecting a number of fluid components. It automatically generates the needed PBSingleJunction components between the specified fluid components. The purpose of this component for user friendliness.

There are only two input parameters required for this component:

- eos (required)

Equation of state to be used for all automatically-generated PBSingleJunctions.

- component_names

This input parameter specifies a list of $N$ sequentially connected fluid components, and $N-1$ PBSingleJunctions will be automatically generated to connect them.

\subsubsection{ChannelCoupling}

ChannelCoupling is a non-geometric component for coupling two 1-D fluid components (with energy exchange). It is intended to model the flow mixing between two parallel channels. 


\begin{tabular}{|c|c|c|}
\hline \multicolumn{3}{|l|}{ [./ChannelCoupling ] } \\
\hline beta & $=($ required $)$ & \# turbulent mixing parameter \\
\hline eos & $=($ required $)$ & \# The name of EOS to use \\
\hline gap_width & $=($ required $)$ & \# The gap width \\
\hline pipe1_name & $=($ no_default $)$ & \# The name of the Pipe 1 \\
\hline pipe2_name & $=($ no_default $)$ & \# The name of the Pipe 2 \\
\hline var_scaling_factor & $=0.001$ & \# turbulent mixing flux variable scaling factor \\
\hline
\end{tabular}

- pipe1_name and pipe2_name

The names of the two pipes where this flow mixing is happening.

- eos (required)

Equation of state to be used in this ChannelCoupling component.

- gap_width (required)

Gap width between the two pipes where this flow mixing is happening.

- beta (required)

This input parameter specifies the turbulent mixing coefficient value to be used to compute the inter-channel mass flux due to turbulent mixing, which will then be used to compute the interchannel energy flux due to turbulent mixing.

- var_scaling_factor

This input parameter specifies the scaling factor for the variable for computing the inter-channel mass flux due to turbulent mixing.

\subsubsection{HeatPipe and HeatPipeArray}

Both components are currently under development, which will be included in future versions of this user's guide.

\subsection{ComponentInputParameters}

Independent to the [Components] input block, SAM also provides a separate [ComponentInputParameters] input block, where users could provide input template for certain types of SAM components. Note that this input block only provides component 'template', and by itself, no real components will created. There are two common usages of this input block: 1) to provide the common features of a type of component, which will be used as reference to build real components in the [Components] input block; and 2) to provide a completely predefined component that will be referred to and created as a sub-component of a composite-type of component. The two common usages are to be discussed in the remaining part of this section. Currently, the SAM components that supports such a feature is listed in Table 4.1.

The first usage is to provide common features for components input. When preparing SAM input files to perform thermal-hydraulics analysis, it is typical to observe that many components share common features, for example, a test loop with the majority of it built from a type of standard 
Table 4.1: SAM components that supports ComponentInputParameters feature

\begin{tabular}{|l|l|}
\hline ComponentInputParameters & SAM Component Name \\
\hline DuctedFuelAssemblyParameters & DuctedFuelAssembly \\
\hline HeatPipeParameters & HeatPipe \\
\hline HeatStructureParameters & HeatStructure \\
\hline MultiChannelRodBundleParameters & MultiChannelRodBundle \\
\hline PBCoreChannelParameters & PBCoreChannel \\
\hline PBOneDFluidComponentParameters & PBOneDFluidComponent \\
\hline PBPipeParameters & PBPipe \\
\hline
\end{tabular}

ASME pipe. In this case, [ComponentInputParameters] can be used to provide the abstracted common features of such a type of components, and [Components] only provides component-specific parameters and refers to these common features to generate the complete input parameter list. With this approach, it greatly reduces users' burden to type in the same parameters for many times, and also reduces the possibility of input error. Note that input parameters specified in the [Components] input blocks can override what has been provided as common features provided in the [ComponentInputParameters] input block. An example is given as follows:

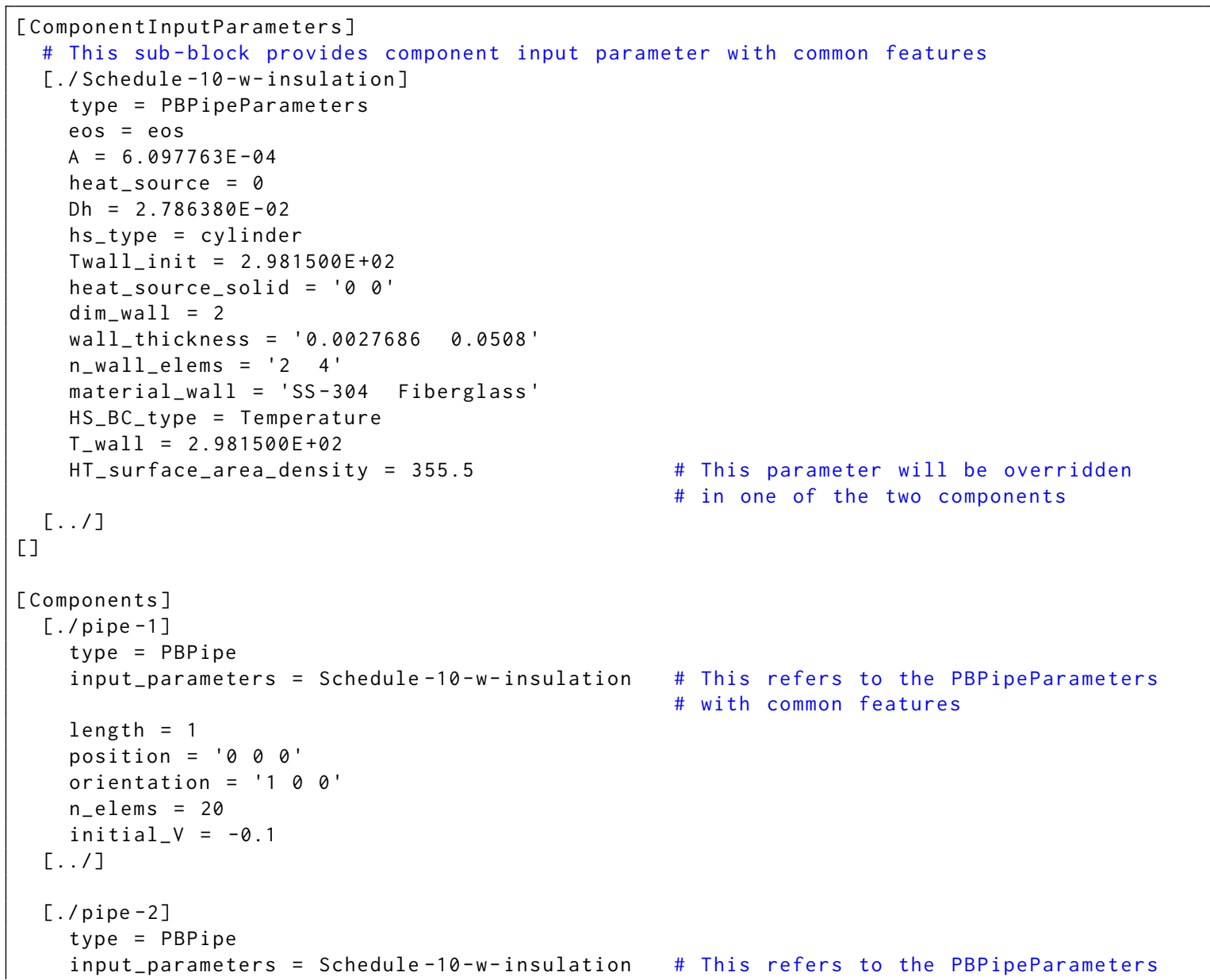


length $=2$

\# with common features

position $=1100$ '

orientation $=' \theta 01$ '

n_elems $=25$

initial_v $=-0.1$

HT_surface_area_density $=100.0 \quad$ \# This parameter overrides what has been

$[. . /]$

\# provided in Schedule-10-w-insulation []

The other usage is to provide needed input parameters, which predefines a component that will be referred to and created as a sub-component of a composite-type of component. Currently, this only happens to a special component, HexLatticeCore, which relies on several predefined components to create its sub-components (see section 4.3.11 for more details). An example is given as follows:

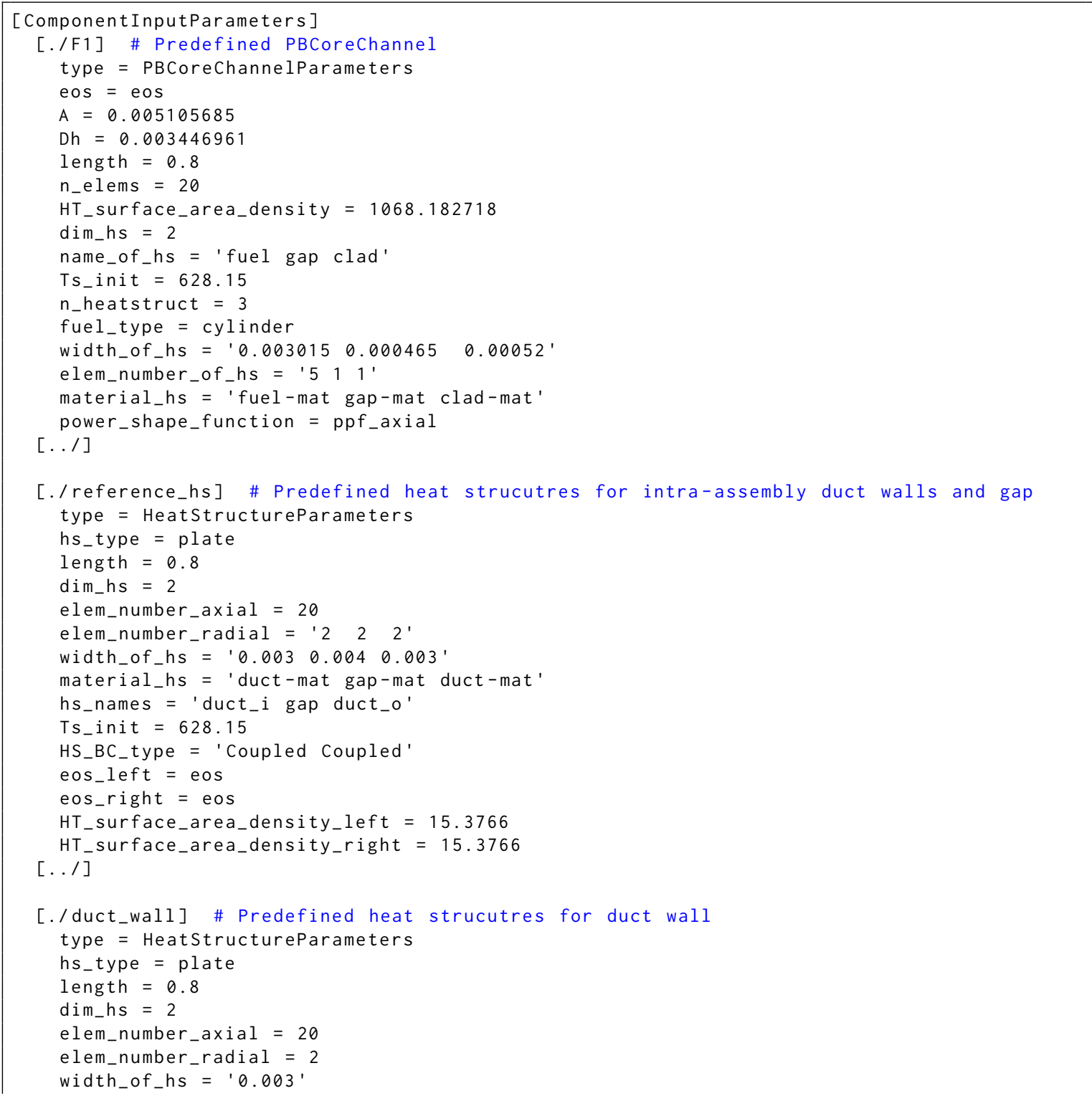




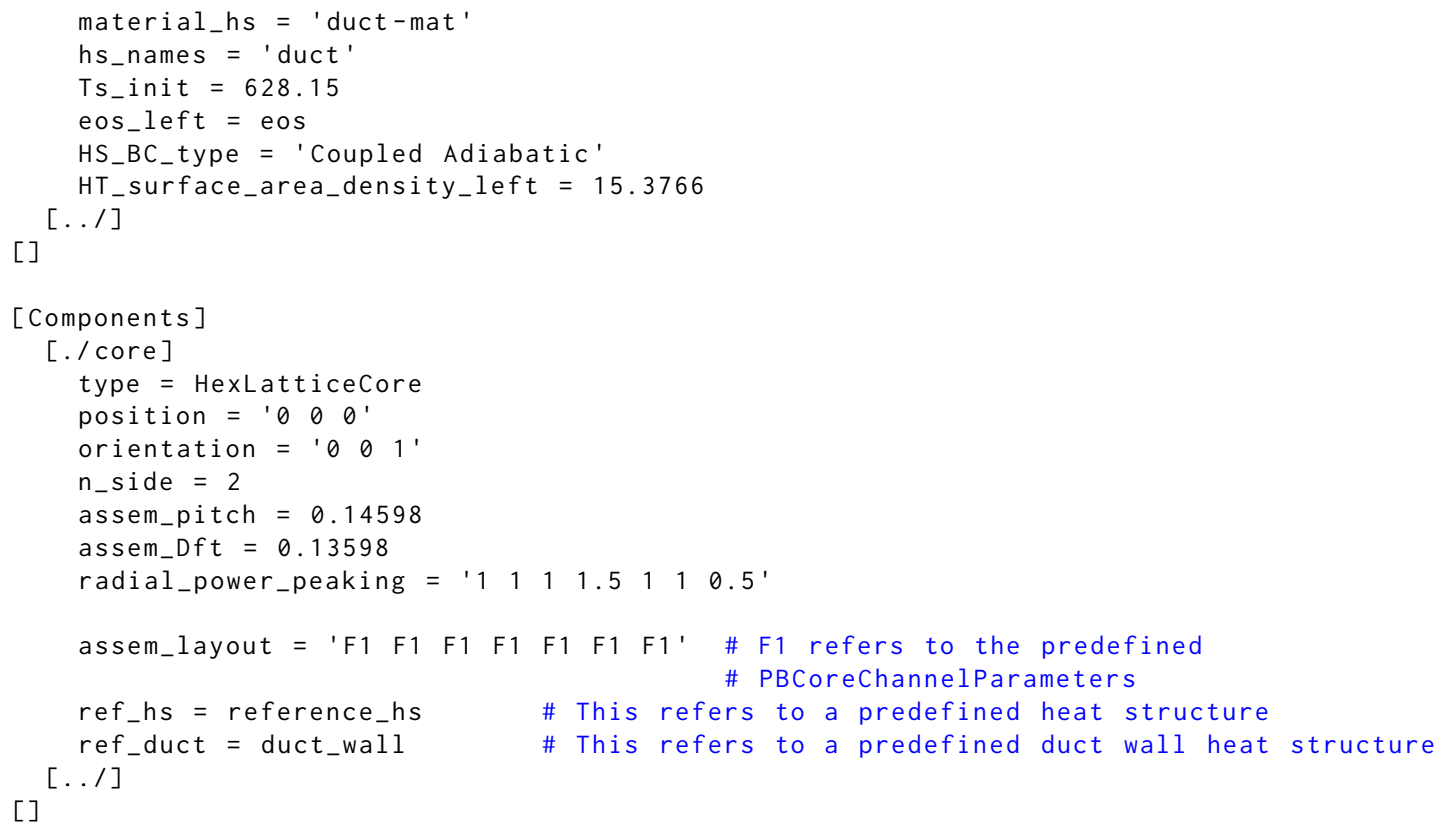

\subsection{PostProcessors}

\subsubsection{ComponentBoundaryEnergyBalance}

This Postprocessor is designed to monitor the energy flux balance between two selected pipe ends. A common usage is to monitor the energy balance of a pipe component on its two ends, and compare it with the total heat source applied to this pipe.

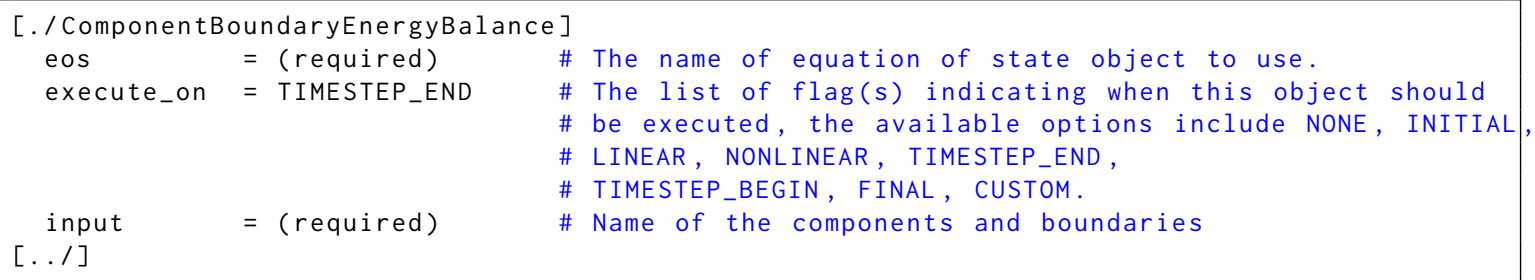

- input (required)

This input parameter specifies a list of two pipe ends, where energy fluxes are to be compared to compute an energy balance between them:

$$
(\rho u h A)_{2}-(\rho u h A)_{1}
$$

The input syntax is similar to those for junction type of component, e.g., input $=$ 'pipe-1(in) pipe-1(out)' or input = 'IHX(secondary_in) IHX(secondary_out)'.

- eos (required)

Equation of state used in the pipe component. 
- execute_on

This is an input parameter inherited from MOOSE framework, it specifies how often this Postprocessor should perform a computation. It is common to all Postprocessors to be discussed in this section, and in general, it is safe to not specify anything.

\subsubsection{ComponentBoundaryFlow}

This Postprocessor is simply monitors the mass flow rate, $\rho u A$, of a pipe end.

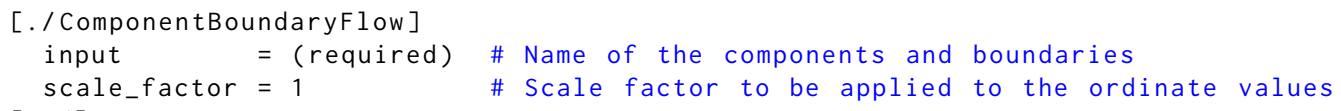

- input (required)

This input parameter specifies a pipe and one of its ends, where mass flow rate is to be computed as $\rho u A$. The input syntax is similar to those for junction type of component, e.g., input = pump_pipe(in).

- scale_factor

This is a scaling factor to be multiplied to the mass flow rate. The default value is 1 .

\subsubsection{ComponentBoundaryScalarFlow}

This Postprocessor is similar to ComponentBoundaryFlow, and it simply monitors the flow rate of a passive scalar, $\rho u A \phi$, of a pipe end.

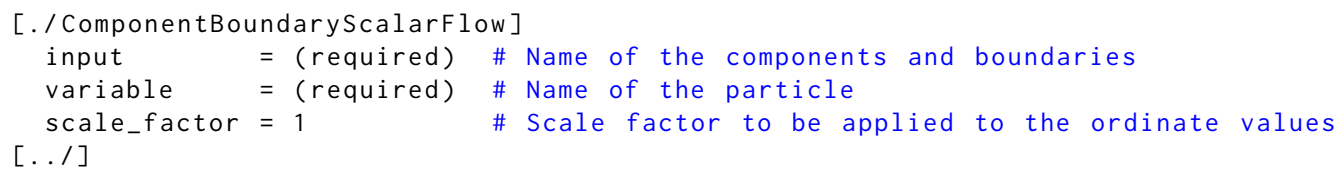

- input (required)

This input parameter specifies a pipe and one of its ends, where the flow rate of a passive scalar is to be computed as $\rho u A \phi$. The input syntax is similar to those for junction type of component, e.g., input = pump_pipe(in).

- variable (required)

The name of the passive scalar variable.

- scale_factor

This is a scaling factor to be multiplied to the flow rate of the passive scalar. The default value is 1. 


\subsubsection{ComponentBoundaryVariableValue}

This Postprocessor returns the value of a specified variable at a pipe end.

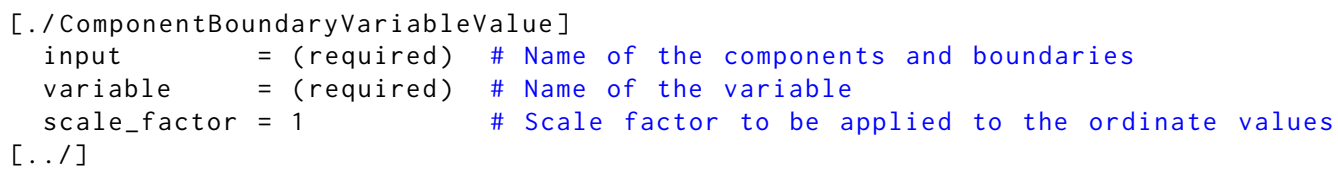

- input (required)

The same as in ComponentBoundaryScalarFlow.

- variable (required)

The name of the variable, such as "pressure", "temperature", "velocity", "rho" (fluid density), "enthalpy", "heat" transfer" coefficient" (if modeled), and passive scalars (if modeled).

- scale_factor

This is a scaling factor to be multiplied to the variable value. The default value is 1 .

\subsubsection{ComponentNodalVariableValue}

This Postprocessor returns the value of a specified variable on a specified node in a pipe.

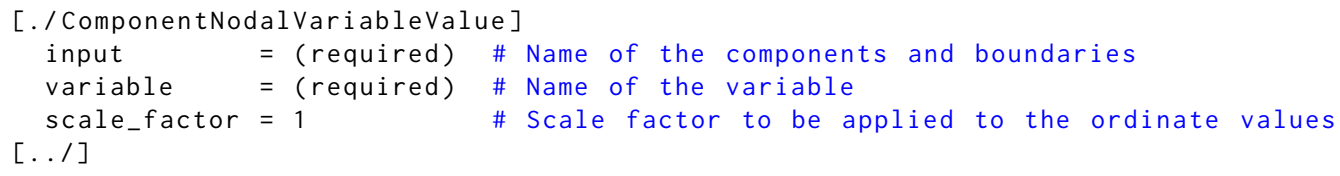

- input (required)

This input parameter specifies a pipe and a node id, where the value of the specified variable will be returned. The input syntax is, for example, input $=$ pipe $(\theta)$ or input $=$ IHX:primary_pipe $(10)$. Note that node id starts from 0.

- variable (required)

The same as in ComponentBoundaryVariableValue.

- scale_factor

This is a scaling factor to be multiplied to the variable value. The default value is 1 .

\subsubsection{ConductionHeatRemovalRate}

This Postprocessor computes the integral heat removal rate from a side of a two-dimensional heat structure.

[. / ConductionHeatRemovalRate]

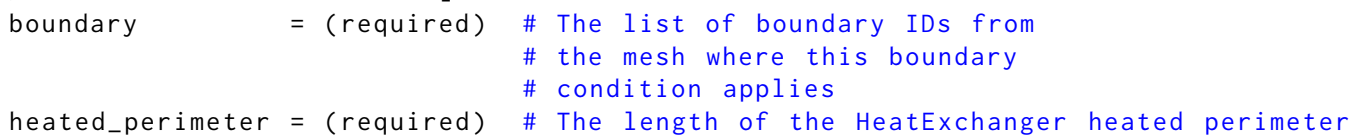


- boundary (required)

This input parameter specifies the boundary name where the integral heat removal rate to be computed, for example, boundary = 'hp 0 : cond_wall'.

- heated_perimeter (required)

The heated perimeter of the boundary to compute the integral heat removal rate,

$$
Q=\int-k \nabla T P_{h} d L
$$

in which, $-k \nabla T$ is the local surface heat flux, $d L$ is the length along the boundary side, and $P_{h}$ is this heated perimeter input parameter.

\subsubsection{HeatExchangerHeatRemovalRate}

This Postprocessor computes the integral heat removal rate from the wall heat structure of a heat exchanger to a specified pipe, e.g., the primary or the secondary side pipe.

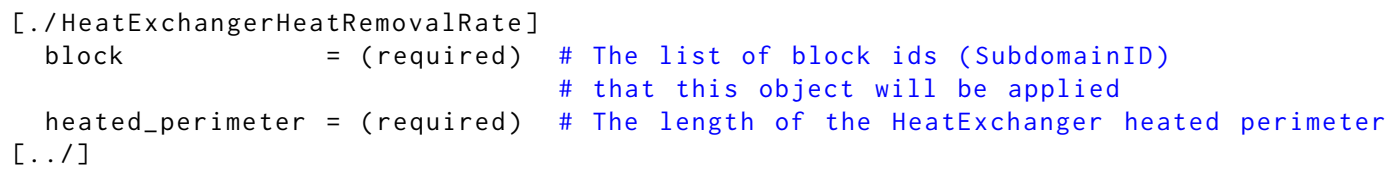

- block (required)

This input parameter specifies the block name where the integral heat removal rate to be computed, for example, block = 'DHX:primary_pipe'.

- heated_perimeter (required)

The heated perimeter of the boundary to compute the integral heat removal rate,

$$
Q=\int h\left(T_{f}-T_{\text {wall }}\right) P_{h} d L
$$

in which, $h$ is the local heat transfer coefficient, $T_{f}$ is the local fluid temperature, $T_{\text {wall }}$ is the local wall surface temperature, $d L$ is the length along the pipe, and $P_{h}$ is this heated perimeter input parameter.

\subsection{TimeSteppers}

\subsubsection{CourantNumberTimeStepper}

The CourantNumberTimeStepper is a TimeStepper inherited from PostprocessorDT, which computes time step size based on a Postprocessor value, in this case, MaxCourantNumber. Its input parameters are listed as follows:

$[. /<$ CourantNumberTimeStepper $>]$

Courant_number $=10$

$\mathrm{dt} \quad=($ no_default $)$

factor $\quad=0$

\# Target Courant number

\# Initial value of $\mathrm{dt}$

\# Add a factor to the

\# supplied postprocessor 


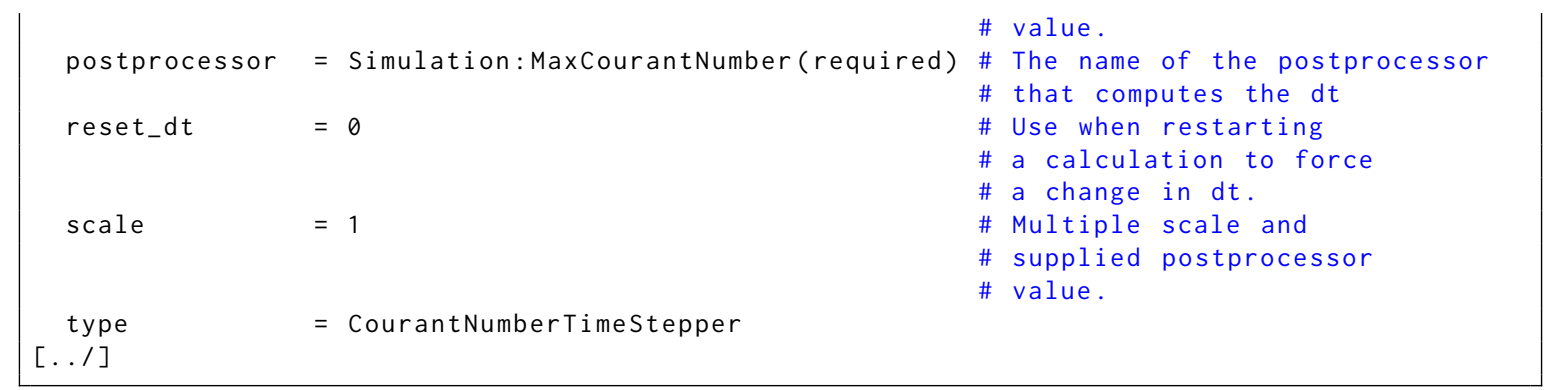

Input parameters are discussed as follows:

- Courant_number

This TimeStepper adjusts the time step size to match this given Courant number as a user input parameter. The default value is 10 .

- $d t$

The initial value of time step size for this TimeStepper to start with. If not specified, the code uses a default value of 0.01 second.

- factor and scale

These two input parameters are not used.

- postprocessor

You do NOT and should NOT specify this input parameter. A default value, Simulation: MaxCourantNumber, has been automatically generated and given to this parameter.

- reset_dt (advanced MOOSE option)

Use when restarting a calculation to force a change in dt. By default, it is false (0).

An example input of the CourantNumberTimeStepper block is shown below.

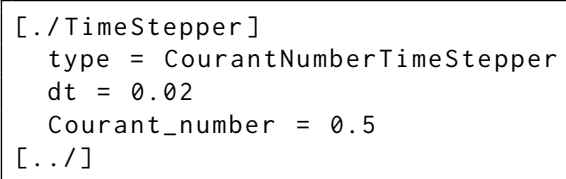

This input block should be used as an sub-block of the Executioner input block.

\subsection{Preconditioning}

The Preconditioning block describes the preconditioner to be used by the preconditioned JFNK solver (available through PETSc). Two options are currently available, the single matrix preconditioner (SMP) and the finite difference preconditioner (FDP). The FDP option uses numerical Jacobian by doing direct finite differences of the residual terms. It is normally slow, and only intended for debugging purposes. The SMP option is more efficient and the recommended option. The input parameters of the Preconditioning block are shown below. An example input block follows. 


\begin{tabular}{|c|c|c|c|}
\hline \multicolumn{4}{|l|}{$\begin{array}{l}{[\text { Preconditioning }]} \\
\qquad[. / *]\end{array}$} \\
\hline active & & _- all__- & $\begin{array}{l}\text { \# If specified only the blocks named will be } \\
\text { \# visited and made active }\end{array}$ \\
\hline line_search & & $=$ default & $\begin{array}{l}\text { \# Specifies the line search type (Note: } \\
\text { \# none = basic) }\end{array}$ \\
\hline petsc_options & & $=$ & \# Singleton PETSc options \\
\hline petsc_options_iname & & $=$ & \# Names of PETSc name/value pairs \\
\hline petsc_options_value & & $=$ & $\begin{array}{l}\text { \# Values of PETSc name/value pairs (must } \\
\text { \# correspond with "petsc_options_iname" }\end{array}$ \\
\hline solve_type & & $=$ & $\begin{array}{l}\text { \# PJFNK: Preconditioned Jacobian-Free Newton } \\
\text { \# Krylov JFNK, NEWTON, FD, LINEAR }\end{array}$ \\
\hline \multicolumn{4}{|r|}{ 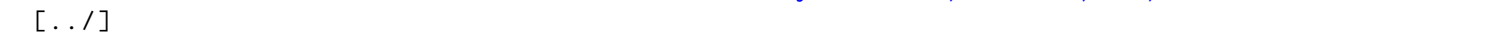 } \\
\hline \multicolumn{4}{|l|}{$[. / F D P]$} \\
\hline control_tags & & $=$ & $\begin{array}{l}\text { \# Adds user-defined labels for accessing } \\
\text { \# object parameters via control logic. }\end{array}$ \\
\hline enable & & $=1$ & \# Set the enabled status of the Mooseobject. \\
\hline full & & $=0$ & $\begin{array}{l}\text { \# Set to true if you want the full set of } \\
\text { \# couplings. }\end{array}$ \\
\hline implicit_geometric_co & oupling & $=0$ & $\begin{array}{l}\text { \# Set to true if you want to add entries into } \\
\text { \# the matrix for degrees of freedom that might } \\
\text { \# be coupled by inspection of the geometric } \\
\text { \# search objects. }\end{array}$ \\
\hline line_search & & $=$ default & $\begin{array}{l}\text { \# Specifies the line search type (Note: } \\
\text { \# none = basic) }\end{array}$ \\
\hline off_diag_column & & $=$ & $\begin{array}{l}\text { \# The off diagonal column you want to add into } \\
\text { \# the matrix, it will be associated with an } \\
\text { \# off diagonal row from the same position in } \\
\text { \# off_diag_row. }\end{array}$ \\
\hline off_diag_row & & $=$ & $\begin{array}{l}\text { \# The off diagonal row you want to add into } \\
\text { \# the matrix, it will be associated } \\
\text { \# with an off diagonal column from the same } \\
\text { \# position in off_diag_colum. }\end{array}$ \\
\hline pc_side & & $=r i g h t$ & \# Preconditioning side \\
\hline petsc_options & & $=$ & \# Singleton PETSC options \\
\hline petsc_options_iname & & $=$ & \# Names of PETSc name/value pairs \\
\hline petsc_options_value & & $=$ & $\begin{array}{l}\text { \# Values of PETSC name/value pairs (must } \\
\text { \# correspond with "petsc_options_iname" }\end{array}$ \\
\hline solve_type & & $=$ & $\begin{array}{l}\text { \# PJFNK: Preconditioned Jacobian-Free Newton } \\
\text { \# Krylov JFNK, NEWTON, FD, LINEAR }\end{array}$ \\
\hline $\begin{array}{l}\text { type } \\
{[\ldots /]}\end{array}$ & & $=\mathrm{FDP}$ & \\
\hline$[. /$ SMP $]$ & & & \\
\hline control_tags & $=$ & & $\begin{array}{l}\text { \# Adds user-defined labels for accessing } \\
\text { \# object parameters via control logic. }\end{array}$ \\
\hline coupled_groups & $=$ & & $\begin{array}{l}\text { \# List multiple space separated groups of } \\
\text { \# comma separated variables. Off-diagonal } \\
\text { \# jacobians will be generated for all pairs } \\
\text { \# within a group. }\end{array}$ \\
\hline enable & $=1$ & & \# Set the enabled status of the MooseObject. \\
\hline full & $=0$ & & $\begin{array}{l}\text { \# Set to true if you want the full set of } \\
\text { \# couplings. }\end{array}$ \\
\hline line_search & $=$ default & & $\begin{array}{l}\text { \# Specifies the line search type (Note: } \\
\text { \# none = basic) }\end{array}$ \\
\hline off_diag_column & $=$ & & $\begin{array}{l}\text { \# The off diagonal column you want to add into } \\
\text { \# the matrix, it will be associated with an } \\
\text { \# off diagonal row from the same position in } \\
\text { \# off_diag_row. }\end{array}$ \\
\hline off_diag_row & $=$ & & $\begin{array}{l}\text { \# The off diagonal row you want to add into } \\
\text { \# the matrix, it will be associated } \\
\text { \# with an off diagonal column from the same }\end{array}$ \\
\hline
\end{tabular}




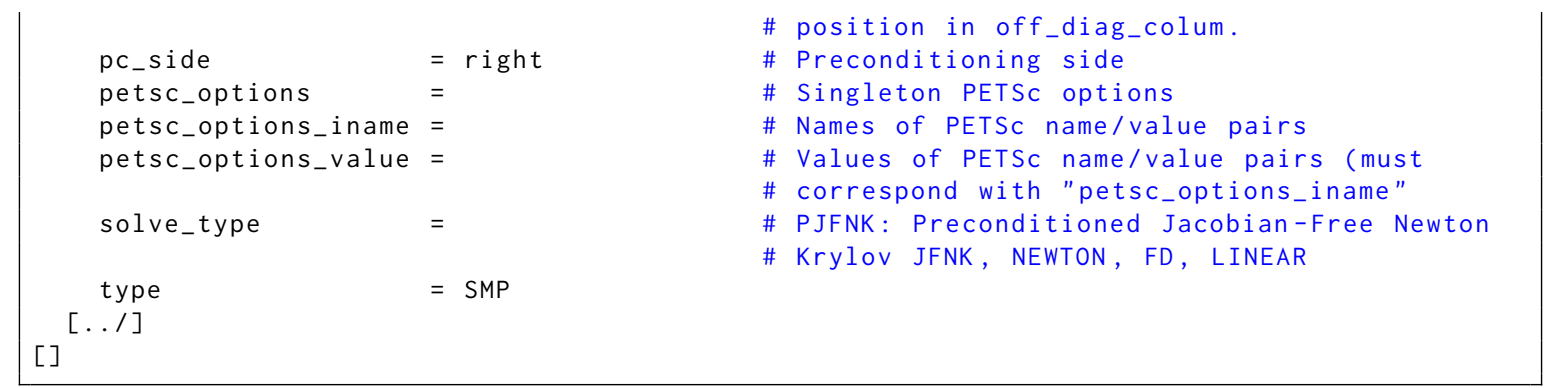

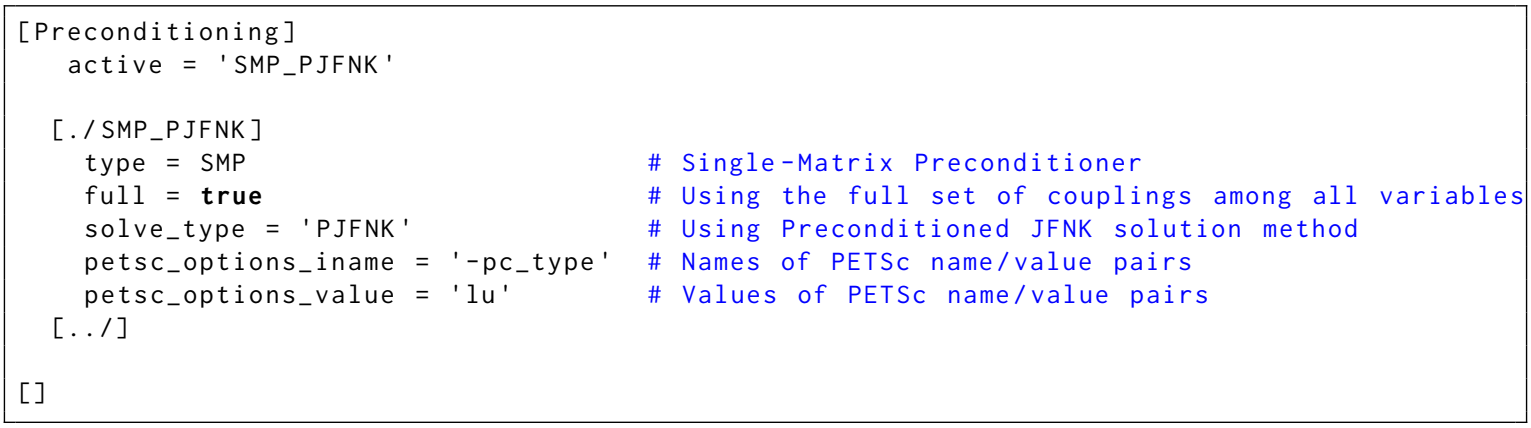

\subsection{Executioner}

The Executioner block describes the calculation process flow used in the simulation. The common MOOSE Executioners are also listed here, and the associated input parameters of the Executioner block are shown below. An example of the Executioner input block is also followed. Common SAM MOOSE Executioner types include: CoupledCFDExecutioner (for coupled simulation with CFD codes), CoupledSASTransient (for coupled SAS/SAM transient simulations), Steady (for steady state simulation), and Transient (for transient simulations).

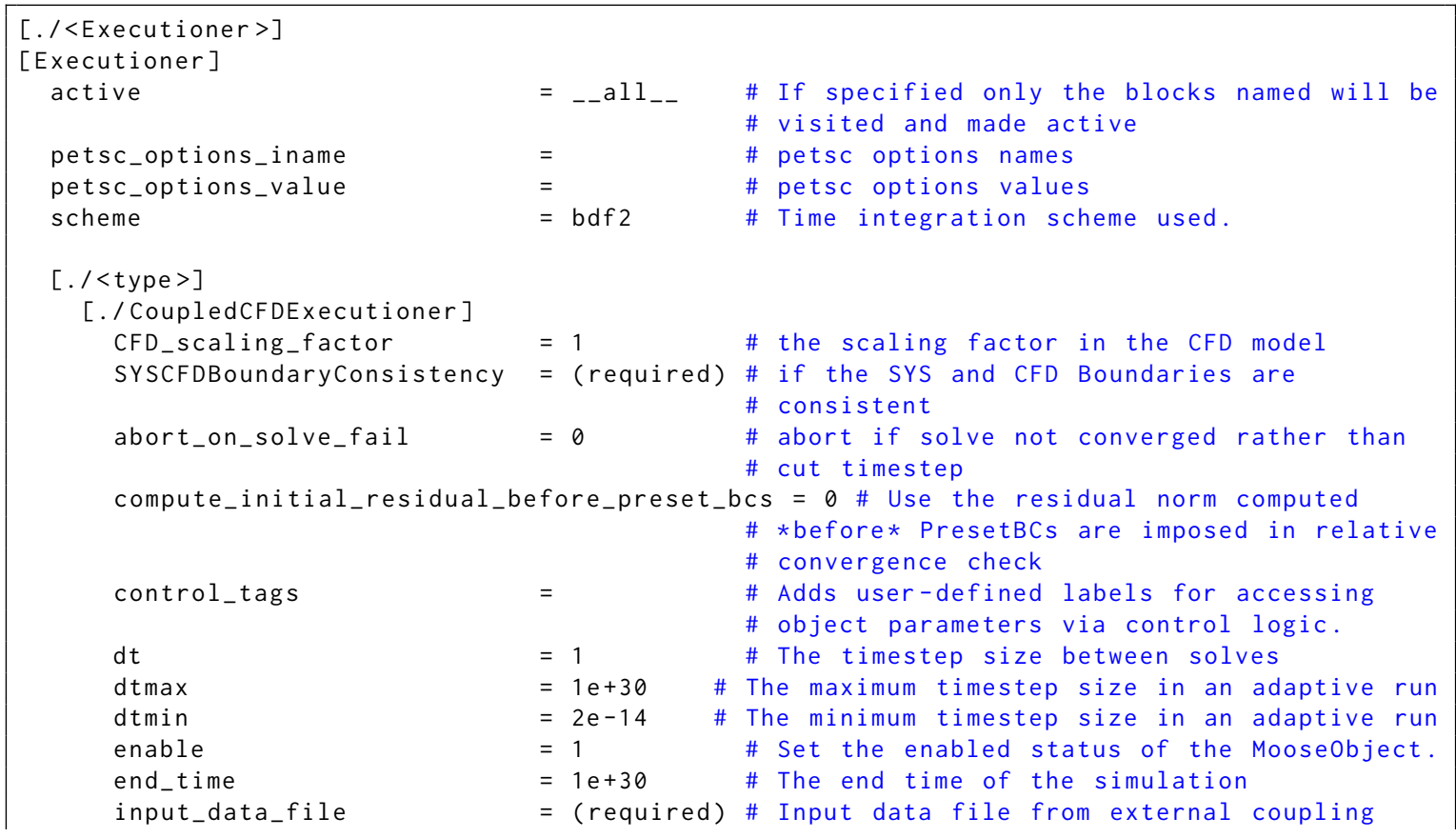




\author{
isRestarting \\ l_abs_step_tol \\ $l_{-}$max_its \\ 1_tol \\ line_search \\ n_in_parameter \\ n_out_parameter \\ n_startup_steps \\ name_of_in_components \\ name_of_in_parameters \\ name_of_out_components \\ name_of_out_parameters \\ names_of_CFD_boundary \\ $n l_{-} a b s_{-}$step_tol \\ nl_abs_tol \\ nl_max_funcs \\ nl_max_its \\ nl_rel_step_tol \\ nl_rel_tol \\ no_fe_reinit \\ num_steps \\ output_data_file \\ petsc_options \\ petsc_options_iname \\ petsc_options_value \\ picard_abs_tol
}

picard_max_its

picard_rel_tol

reset_dt

restart_file_base

scheme

solve_type

splitting

ss_check_tol

ss_tmin

start_time

time_period_ends

time_period_starts

time_periods

timestep_tolerance

trans_ss_check

$$
\begin{aligned}
& =0 \\
& =-1 \\
& =10000 \\
& =1 \mathrm{e}-05 \\
& =\text { default } \\
& =\text { (require } \\
& =\text { (require } \\
& =0 \\
& =\text { (require } \\
& =\text { (require } \\
& =\text { (require } \\
& =\text { (require }) \\
& =\text { (require }) \\
& =1 \mathrm{e}-50 \\
& =1 \mathrm{e}-50 \\
& =10000 \\
& =50 \\
& =1 \mathrm{e}-50 \\
& =1 \mathrm{e}-08 \\
& =0
\end{aligned}
$$

\# if it is a restart coupled code simulation \# Linear Absolute Step Tolerance

\# Max Linear Iterations

\# Linear Tolerance

\# Specifies the line search type

\# (Note: none = basic)

$=$ (required) \# Number of coupling input parameters

= (required) \# Number of coupling output parameters \# The number of timesteps during startup

$=$ (required) \# Names of coupling input components

= (required) \# Parameter names of coupling input \# components

= (required) \# Names of coupling output components

= (required) \#Variable names of coupling output \# components

= (required) \# names of coupled CFD boundaries

$=1 e-50$ \# Nonlinear Absolute step Tolerance

$=1 \mathrm{e}-50$ \# Nonlinear Absolute Tolerance

$=10000$ \# Max Nonlinear solver function evaluations

\# Max Nonlinear Iterations

\# Nonlinear Relative step Tolerance

\# Nonlinear Relative Tolerance

\# Specifies whether or not to reinitialize

\# FEs

$=4294967295$ \# The number of timesteps in a transient run

= (required) \# Output data file for external coupling

$=\quad$ \# Singleton PETSc options

$=\quad$ \# Names of PETSc name/value pairs

$=\quad$ \# Values of PETSc name/value pairs (must

\# correspond with "petsc_options_iname"

\# The absolute nonlinear residual to shoot

\# for during Picard iterations. This check is

\# performed based on the Master app's

\# nonlinear residual.

$=1$ \# Number of times each timestep will be

\# solved. Mainly used when wanting to do

\# Picard iterations with MultiApps that

\# are set to execute_on

\# timestep_end or timestep_begin

$=1 \mathrm{e}-08$ \# The relative nonlinear residual drop

\# to shoot for during Picard iterations.

\# This check is performed based on the Master

\# app's nonlinear residual.

$=0 \quad$ \# Use when restarting a calculation to force

\# a change in $d t$.

\# File base name used for restart

\# Time integration scheme used.

\# PJFNK: Preconditioned Jacobian-Free Newton

\# Krylov JFNK, NEWTON, FD, LINEAR

\# Top-level splitting defining a hierarchical

\# decomposition into subsystems to help

\# the solver.

$=1 \mathrm{e}-08$ \# Whenever the relative residual changes by

\# less than this the solution

\# will be considered to be at steady state.

\# Minimum number of timesteps to take before

\# checking for steady state conditions.

\# The start time of the simulation

\# The end times of time periods

\# The start times of time periods

\# The names of periods

\# the tolerance setting for final timestep

\# size and sync times

\# Whether or not to check for steady state 


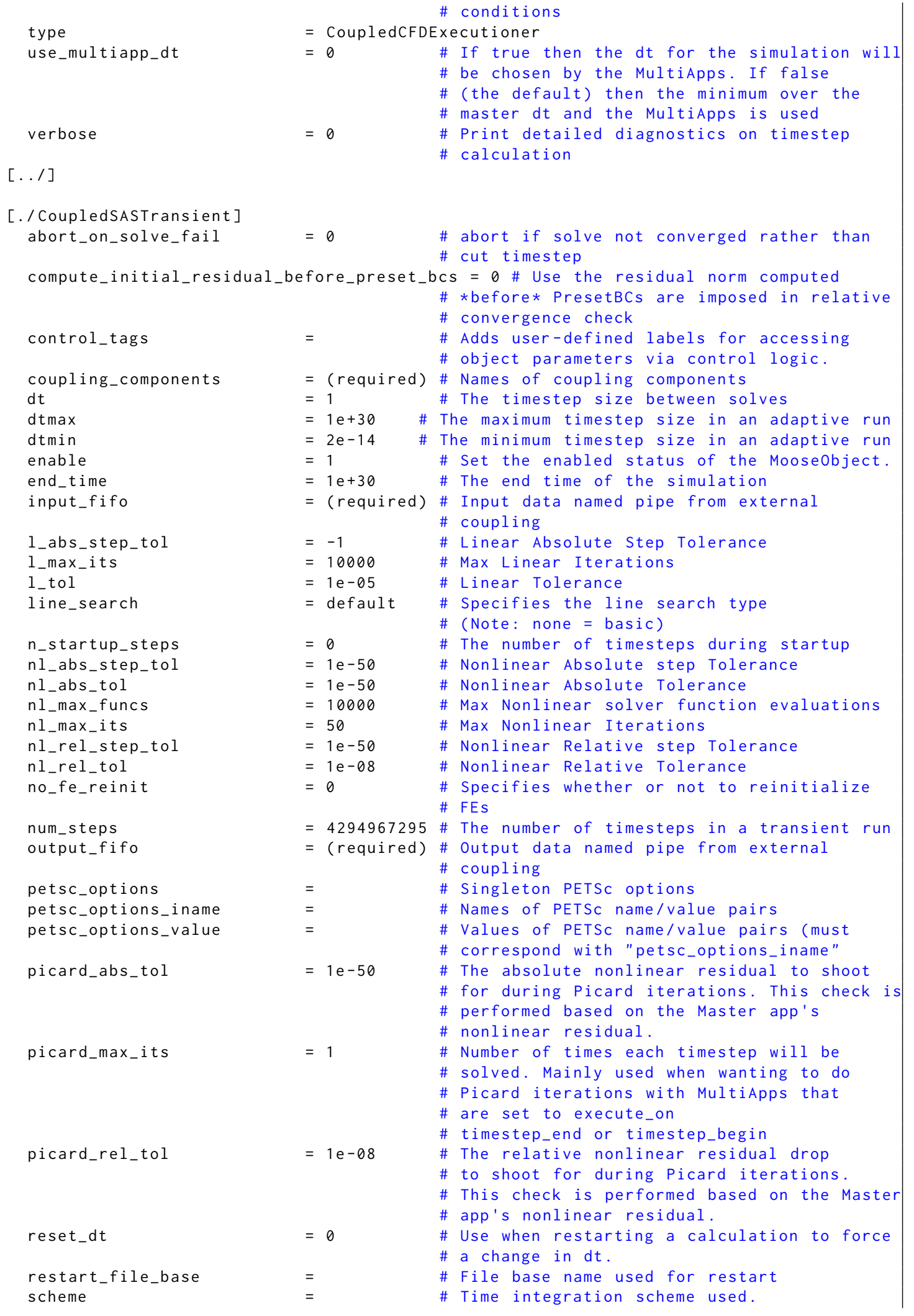




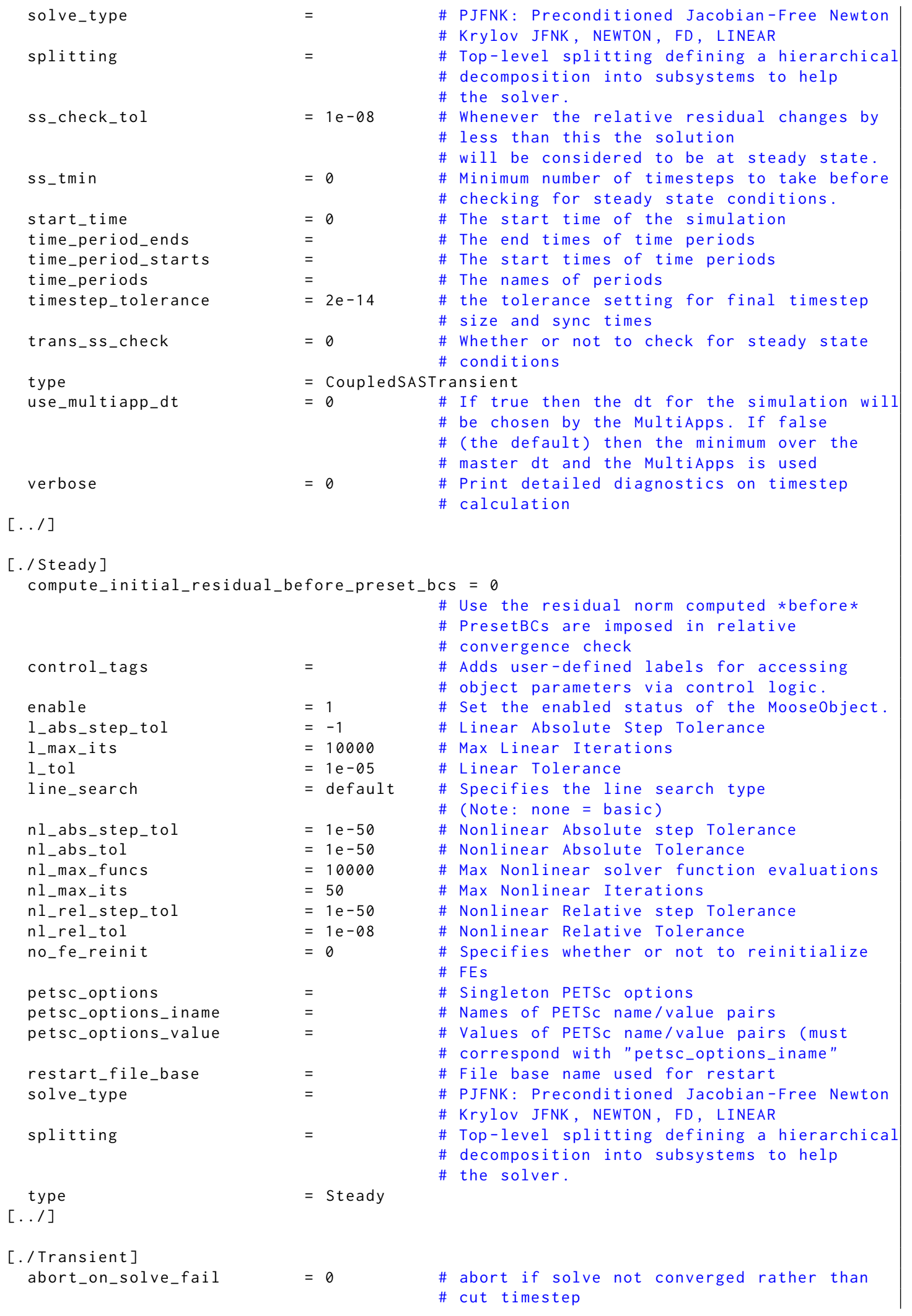




\author{
control_tags \\ $d t$ \\ dtmax \\ dtmin \\ enable \\ end_time \\ 1_abs_step_tol \\ $1_{-} \mathrm{max}_{-}$its \\ 1_tol \\ line_search \\ n_startup_steps \\ nl_abs_step_tol \\ nl_abs_tol \\ nl_max_funcs \\ nl_max_its \\ nl_rel_step_tol \\ nl_rel_tol \\ no_fe_reinit \\ num_steps \\ petsc_options \\ petsc_options_iname \\ petsc_options_value \\ picard_abs_tol
}

picard_max_its

picard_rel_tol

reset_dt

restart_file_base

scheme

solve_type

splitting

ss_check_tol

ss_tmin

start_time

time_period_ends

time_period_starts

time_periods

timestep_tolerance

trans_ss_check

type

use_multiapp_dt
$=1$

$=1 \mathrm{e}+30$

$=2 \mathrm{e}-14$

$=1$

$=1 e+30$

$=-1$

$=10000$

$=1 \mathrm{e}-05$

= default

$=0$

$=1 \mathrm{e}-50$

$=1 \mathrm{e}-50$

$=10000$

$=50$

$=1 \mathrm{e}-50$

$=1 \mathrm{e}-08$

$=0$

4294967295

$=$

$=$

$=$

$=1 \mathrm{e}-50$

$=1$

$=1 \mathrm{e}-08$

$=0$

$=$

$=$

$=$

$=$

$=1 \mathrm{e}-08$

$=0$

$=0$

$=$

$=$

$=$

$=2 \mathrm{e}-14$

$=0$

$=$ Transient

$=0$
\# Adds user-defined labels for accessing

\# object parameters via control logic.

\# The timestep size between solves

\# The maximum timestep size in an adaptive run

\# The minimum timestep size in an adaptive run \# Set the enabled status of the Mooseobject.

\# The end time of the simulation

\# Linear Absolute Step Tolerance

\# Max Linear Iterations

\# Linear Tolerance

\# Specifies the line search type

\# (Note: none = basic)

\# The number of timesteps during startup

\# Nonlinear Absolute step Tolerance

\# Nonlinear Absolute Tolerance

\# Max Nonlinear solver function evaluations

\# Max Nonlinear Iterations

\# Nonlinear Relative step Tolerance

\# Nonlinear Relative Tolerance

\# Specifies whether or not to reinitialize

\# FEs

number of timesteps in a transient run

\# Singleton PETSC options

\# Names of PETSc name/value pairs

\# Values of PETSc name/value pairs (must

\# correspond with "petsc_options_iname"

\# The absolute nonlinear residual to shoot

\# for during Picard iterations. This check is

\# performed based on the Master app's

\# nonlinear residual.

\# Number of times each timestep will be

\# solved. Mainly used when wanting to do

\# Picard iterations with MultiApps that

\# are set to execute_on

\# timestep_end or timestep_begin

\# The relative nonlinear residual drop

\# to shoot for during Picard iterations.

\# This check is performed based on the Master

\# app's nonlinear residual.

\# Use when restarting a calculation to force

\# a change in $d t$.

\# File base name used for restart

\# Time integration scheme used.

\# PJFNK: Preconditioned Jacobian-Free Newton

\# Krylov JFNK, NEWTON, FD, LINEAR

\# Top-level splitting defining a hierarchical

\# decomposition into subsystems to help

\# the solver.

\# Whenever the relative residual changes by

\# less than this the solution

\# will be considered to be at steady state.

\# Minimum number of timesteps to take before

\# checking for steady state conditions.

\# The start time of the simulation

\# The end times of time periods

\# The start times of time periods

\# The names of periods

\# the tolerance setting for final timestep

\# size and sync times

\# Whether or not to check for steady state

\# conditions

\# If true then the dt for the simulation will 


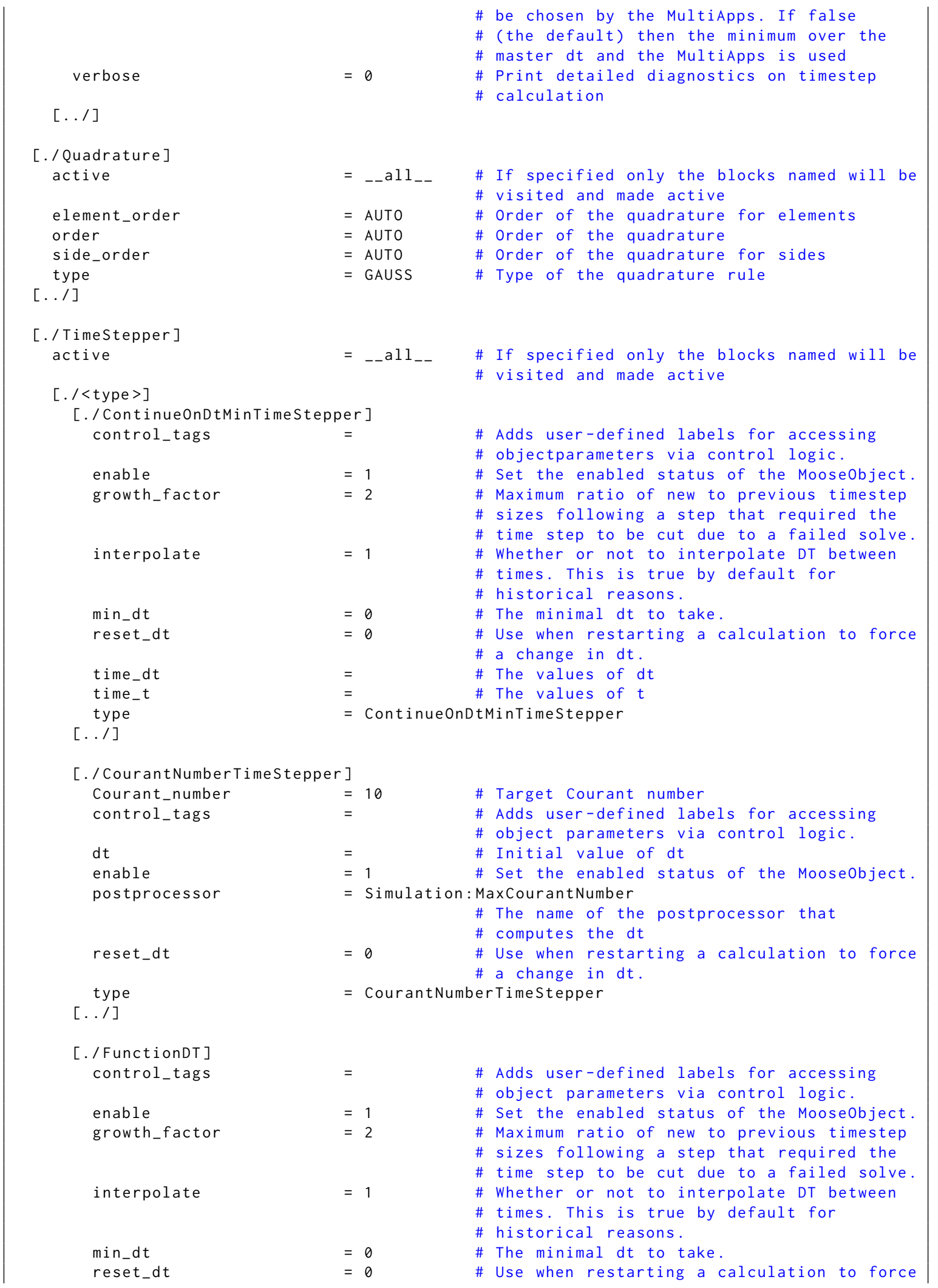




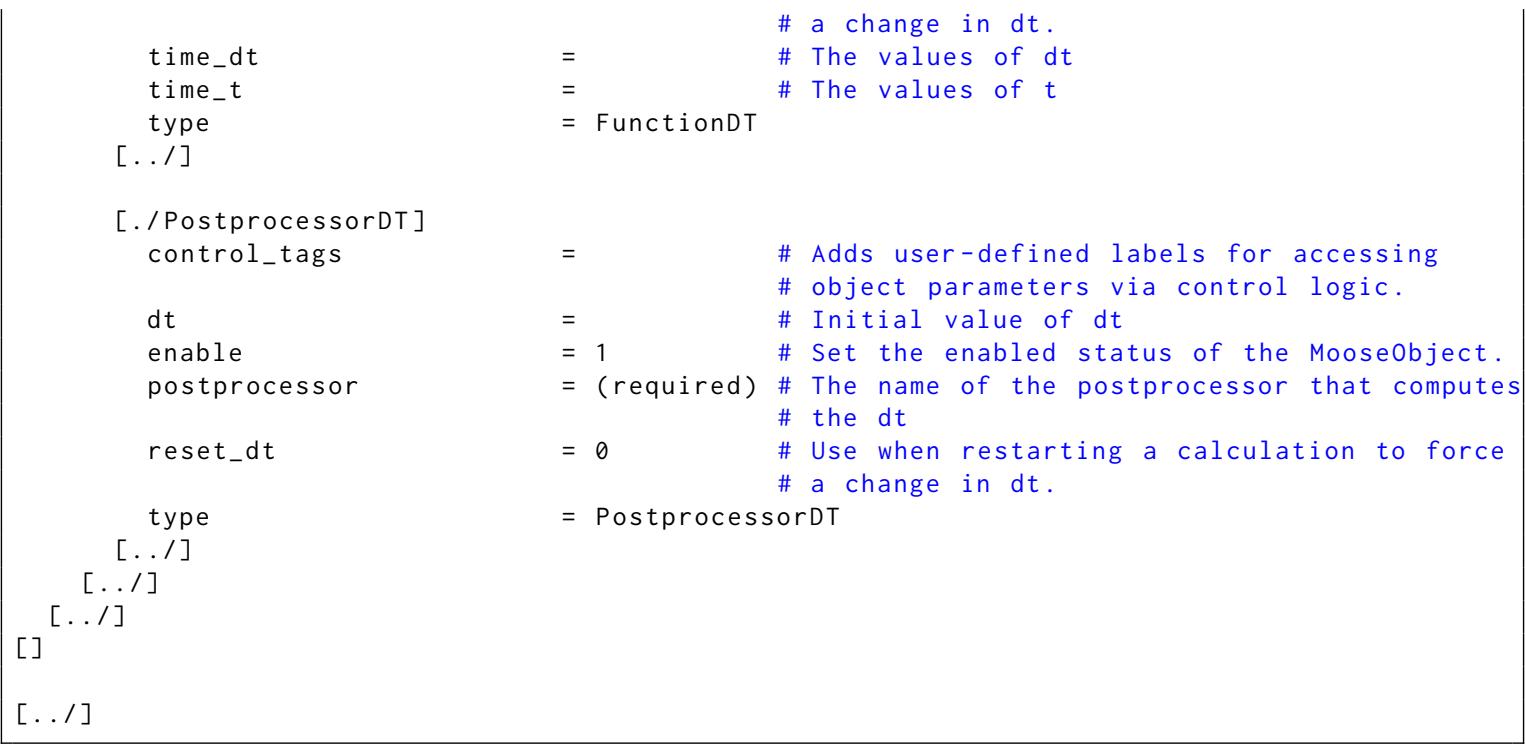

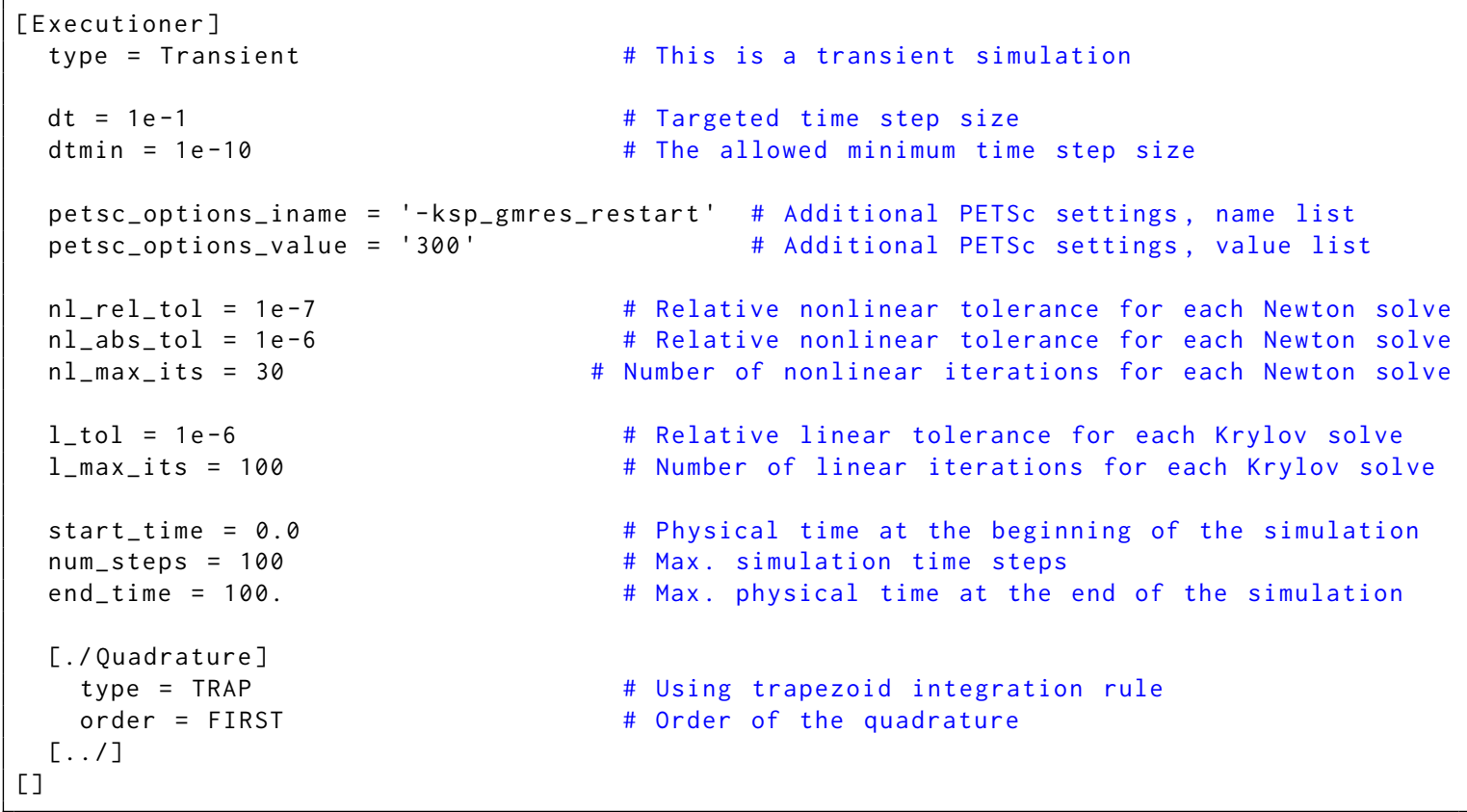

\subsection{Outputs}

The Outputs block specifies various settings of different output types (screen display and files) in the simulation. The input parameters of common MOOSE Outputs are shown below, with an example Outputs block followed. Common MOOSE output types include:

- CSV: write post-processor and scalar variables to a separate comma-separated-values file,

- Checkpoint: save snapshots of the simulation data including all meshes, solutions, and stateful object data, 
- Console: output to screen with runtime information,

- Exocdus: write all mesh and solution data to an ExodusII file.

\begin{tabular}{|c|c|c|}
\hline [Outputs] & & \\
\hline active & $=-{ }_{-}$all $\_-$ & $\begin{array}{l}\text { \# If specified only the blocks named will be } \\
\text { \# visited and made active }\end{array}$ \\
\hline$[. / \mathrm{CSV}]$ & & \\
\hline additional_execute_on & $=$ & $\begin{array}{l}\text { \# This list of output flags is added to the } \\
\text { \# existing flags (initial|linear|nonlinearl } \\
\text { \# timestep_end|timestep_begin|final| } \\
\text { \# failed|custom) }\end{array}$ \\
\hline align & $=0$ & $\begin{array}{l}\text { \# to execute only at that moment } \\
\text { \# Align the outputted csv data by padding } \\
\text { \# the numbers with trailing whitespace }\end{array}$ \\
\hline append_date & $=0$ & $\begin{array}{l}\text { \# When true the date and time are appended } \\
\text { \# to the output filename. }\end{array}$ \\
\hline append_date_format & $=$ & $\begin{array}{l}\text { \# The format of the date/time to append, } \\
\text { \# if not given UTC format used (see } \\
\text { \# http://www. cplusplus.com/reference } \\
\text { \# /ctime/strftime). }\end{array}$ \\
\hline append_restart & $=0$ & \# Append existing file on restart \\
\hline control_tags & $=$ & $\begin{array}{l}\text { \# Adds user-defined labels for accessing } \\
\text { \# object parameters via control logic. }\end{array}$ \\
\hline delimiter & $=$ & \# Assign the delimiter (default is ',' \\
\hline enable & $=1$ & \# Set the enabled status of the Mooseobject. \\
\hline end_time & $=$ & $\begin{array}{l}\text { \# Time at which this output object stop } \\
\text { \# operating }\end{array}$ \\
\hline execute_elemental_variables & $=1$ & $\begin{array}{l}\text { \# Enable/disable the output of elemental } \\
\text { \# variables }\end{array}$ \\
\hline execute_input & $=1$ & $\begin{array}{l}\text { \# Enable/disable the output of input file } \\
\text { \# information }\end{array}$ \\
\hline execute_nodal_variables & $=1$ & $\begin{array}{l}\text { \# Enable/disable the output of nodal } \\
\text { \# variables }\end{array}$ \\
\hline execute_on & $='$ INITIAL & $\begin{array}{l}\text { TIMESTEP_END' \# Set to } \\
\quad \# \text { (none|initial|linear|nonlinear| } \\
\text { \# timestep_end|timestep_begin|final| } \\
\text { \# failed|custom) } \\
\text { \# to execute only at that moment }\end{array}$ \\
\hline execute_postprocessors_on & $=$ & \# Control of when postprocessors are output \\
\hline execute_scalar_variables & $=1$ & $\begin{array}{l}\text { \# Enable/disable the output of aux scalar } \\
\text { \# variables }\end{array}$ \\
\hline execute_scalars_on & $=$ & \# Control the output of scalar variables \\
\hline execute_system_information & $=1$ & $\begin{array}{l}\text { \# Enable/disable the output of the simulation } \\
\text { \# information }\end{array}$ \\
\hline execute_vector_postprocessors & $s=1$ & $\begin{array}{l}\text { \# Enable/disable the output of vector } \\
\text { \# postprocessors }\end{array}$ \\
\hline execute_vector_postprocessors & S_on = & $\begin{array}{l}\text { \# Enable/disable the output of } \\
\text { \#VectorPostprocessors }\end{array}$ \\
\hline file_base & $=$ & $\begin{array}{l}\text { \# The desired solution output name without an } \\
\text { \# extension }\end{array}$ \\
\hline hide & $=$ & $\begin{array}{l}\text { \# A list of the variables and postprocessors } \\
\text { \# that should NOT be output to the Exodus } \\
\text { \# file (may include Variables, } \\
\text { \# ScalarVariables, and Postprocessor names). }\end{array}$ \\
\hline interval & $=1$ & $\begin{array}{l}\text { \# The interval at which time steps are output } \\
\text { \# to the solution file }\end{array}$ \\
\hline linear_residual_dt_divisor & $=1000$ & $\begin{array}{l}\text { \# Number of divisions applied to time step } \\
\text { \# when outputting linear residuals }\end{array}$ \\
\hline linear_residual_end_time & $=$ & $\begin{array}{l}\text { \# Specifies an end time to begin output on } \\
\text { \# each linear residual evaluation }\end{array}$ \\
\hline linear_residual_start_time & $=$ & $\begin{array}{l}\text { \# Specifies a start time to begin output on } \\
\text { \# each linear residual evaluation }\end{array}$ \\
\hline
\end{tabular}




\begin{tabular}{|c|c|c|}
\hline linear_residuals & $=0$ & $\begin{array}{l}\text { \# Specifies whether output occurs on each } \\
\text { \# linear residual evaluation }\end{array}$ \\
\hline nonlinear_residual_dt_divisor & $=1000$ & $\begin{array}{l}\text { \# Number of divisions applied to time step } \\
\text { \# when outputting non-linear residuals }\end{array}$ \\
\hline nonlinear_residual_end_time & $=$ & $\begin{array}{l}\text { \# Specifies an end time to begin output on } \\
\text { \# each nonlinear residual evaluation }\end{array}$ \\
\hline nonlinear_residual_start_time & $=$ & $\begin{array}{l}\text { \# Specifies a start time to begin output on } \\
\text { \# each nonlinear residual evaluation }\end{array}$ \\
\hline nonlinear_residuals & $=0$ & $\begin{array}{l}\text { \# Specifies whether output occurs on each } \\
\text { \# nonlinear residual evaluation }\end{array}$ \\
\hline output_if_base_contains & $=$ & $\begin{array}{l}\text { \# If this is supplied then output will only } \\
\text { \# be done in the case that the output base } \\
\text { \# contains one of these strings. This is } \\
\text { \# helpful in outputting only a subset of } \\
\text { \# outputs when using Multiapps. }\end{array}$ \\
\hline output_linear & $=0$ & $\begin{array}{l}\text { \# Specifies whether output occurs on each } \\
\text { \# linear residual evaluation }\end{array}$ \\
\hline output_nonlinear & $=0$ & $\begin{array}{l}\text { \# Specifies whether output occurs on each } \\
\text { \# nonlinear residual evaluation }\end{array}$ \\
\hline output_postprocessors & $=1$ & \# Enable/disable the output of postprocessors \\
\hline precision & $=14$ & \# Set the output precision \\
\hline show & $=$ & $\begin{array}{l}\text { \# A list of the variables and postprocessors } \\
\text { \# that should be output to the Exodus file } \\
\text { \# (may include Variables, ScalarVariables, } \\
\text { \# and Postprocessor names). }\end{array}$ \\
\hline start_time & $=$ & $\begin{array}{l}\text { \# Time at which this output object begins to } \\
\text { \# operate }\end{array}$ \\
\hline sync_only & $=0$ & \# Only export results at sync times \\
\hline sync_times & $=$ & $\begin{array}{l}\text { \# Times at which the output and solution is } \\
\text { \# forced to occur }\end{array}$ \\
\hline time_data & $=0$ & $\begin{array}{l}\text { \# When true and VecptorPostprocessor data } \\
\text { \# exists, write a csv file containing } \\
\text { \# the timestep and time information. }\end{array}$ \\
\hline time_tolerance & $=1 \mathrm{e}-14$ & $\begin{array}{l}\text { \# Time tolerance utilized checking start and } \\
\text { \# end times }\end{array}$ \\
\hline type & $=\mathrm{CSV}$ & \\
\hline use_displaced & $=0$ & $\begin{array}{l}\text { \# Enable/disable the use of the displaced } \\
\text { \# mesh for outputting }\end{array}$ \\
\hline .1$]$ & & \\
\hline & & \\
\hline additional_execute_on & $=$ & $\begin{array}{l}\text { \# This list of output flags is added to the } \\
\text { \# existing flags (initial|linear|nonlinearl } \\
\text { \# timestep_end|timestep_begin|final| } \\
\text { \# failed|custom) } \\
\text { \# to execute only at that moment }\end{array}$ \\
\hline append_date & $=0$ & $\begin{array}{l}\text { \# When true the date and time are appended } \\
\text { \# to the output filename. }\end{array}$ \\
\hline append_date_format & $=$ & $\begin{array}{l}\text { \# The format of the date/time to append, } \\
\text { \# if not given UTC format used (see } \\
\text { \# http://www.cplusplus.com/reference } \\
\text { \# /ctime/strftime). }\end{array}$ \\
\hline binary & $=1$ & \# Toggle the output of binary files \\
\hline control_tags & $=$ & $\begin{array}{l}\text { \# Adds user-defined labels for accessing } \\
\text { \# object parameters via control logic. }\end{array}$ \\
\hline enable & $=1$ & \# Set the enabled status of the Mooseobject. \\
\hline end_time & $=$ & $\begin{array}{l}\text { \# Time at which this output object stop } \\
\text { \# operating }\end{array}$ \\
\hline execute_on & $=$ 'INITIAL & $\begin{array}{l}\text { TIMESTEP_END } \quad \text { \# Set to } \\
\quad \# \text { (none|initial|linear|nonlinear | } \\
\text { \# timestep_end|timestep_begin|final| } \\
\text { \# failed|custom) }\end{array}$ \\
\hline file_base & $=$ & $\begin{array}{l}\text { \# to execute only at that moment } \\
\text { \# The desired solution output name without ar }\end{array}$ \\
\hline
\end{tabular}




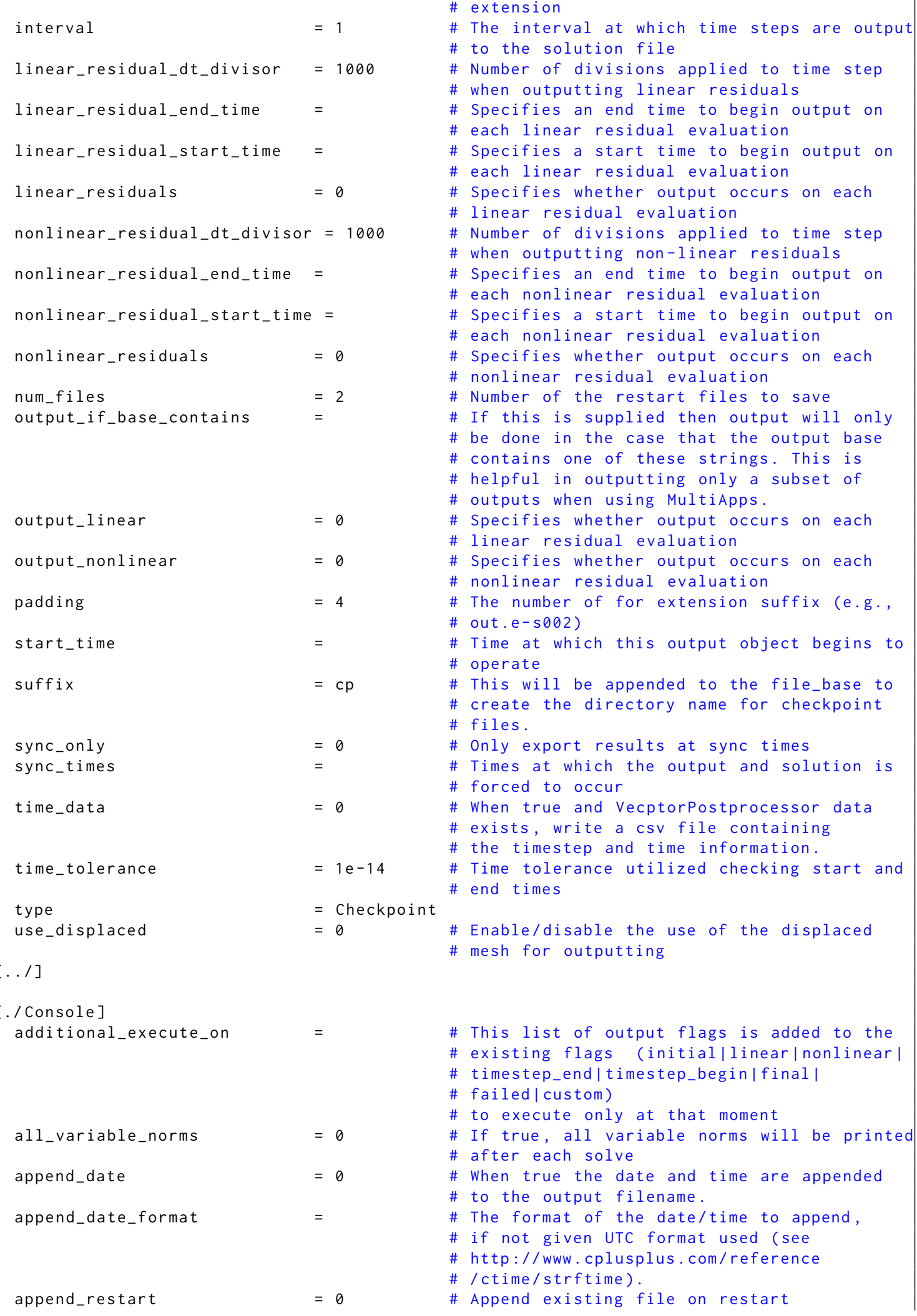




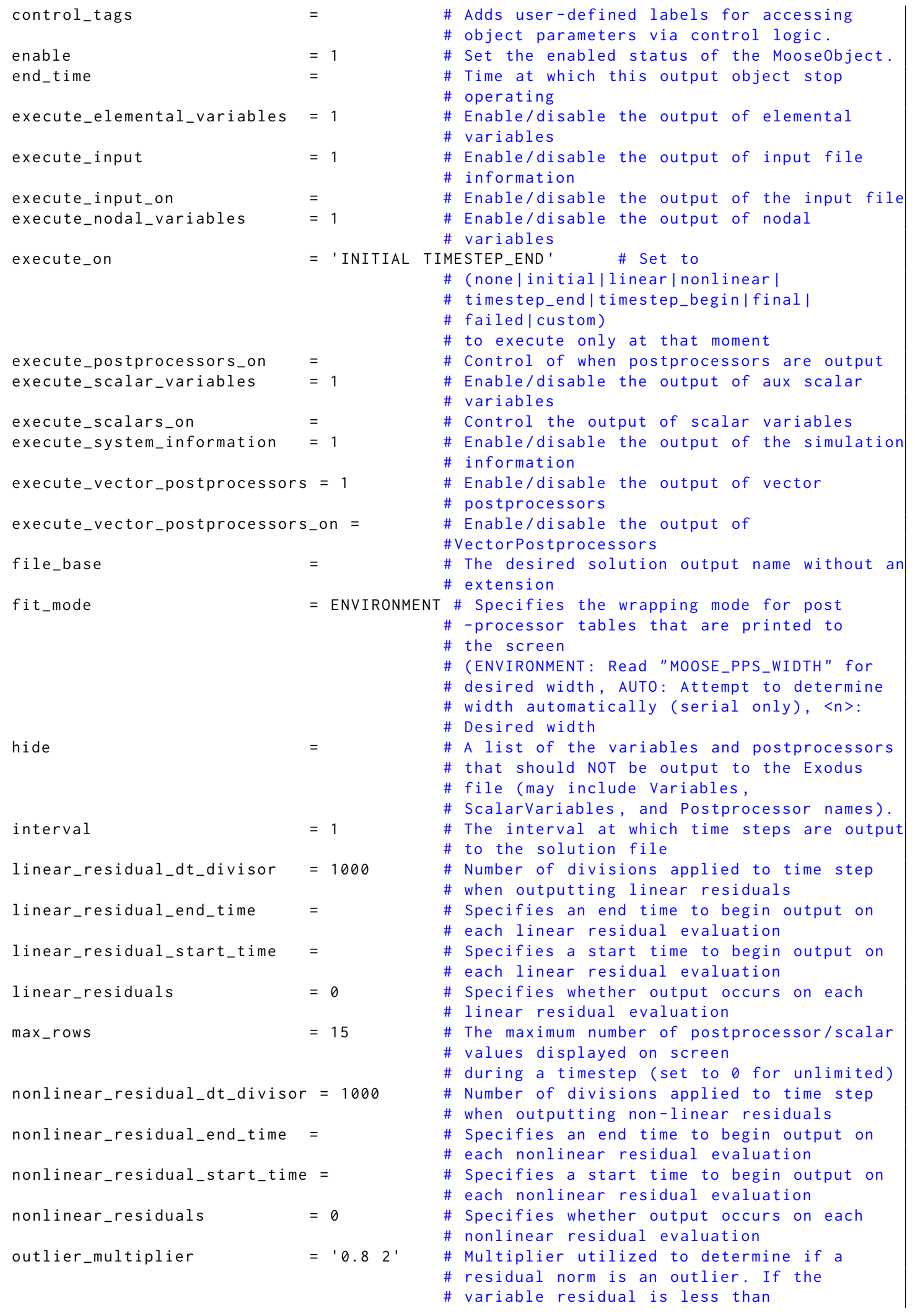




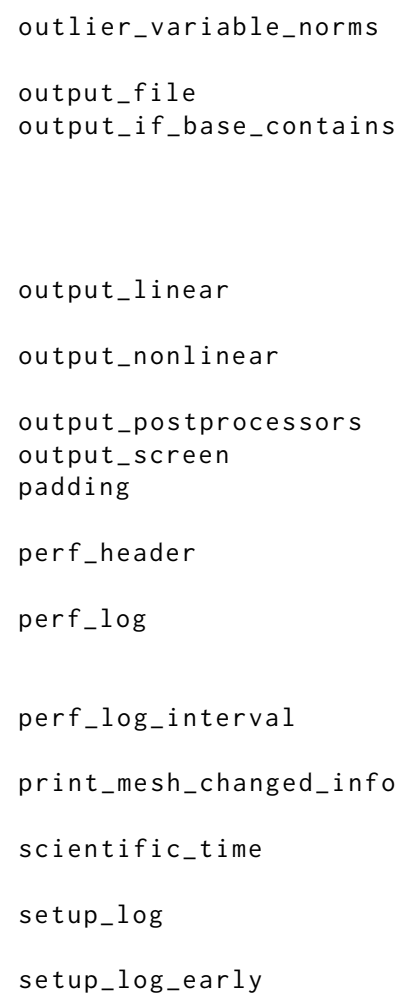

= 'AUX EXECUTION FRAMEWORK MESH NONLINEAR'

\# List of information types

\# to display ('framework', 'mesh', 'aux',

\# 'nonlinear', 'execution', 'output')

$=0 \quad$ \# When true and VecptorPostprocessor data

\# exists, write a csv file containing

\# the timestep and time information.

$=\quad$ \# The number of significant digits that are

\# printed on time related outputs

$=1 \mathrm{e}-14 \quad \#$ Time tolerance utilized checking start and \# end times 
type

use_displaced

verbose

$[\ldots /]$

[./Exodus]

additional_execute_on

append_date

append_date_format

append_oversample

control_tags

enable

end_time

execute_elemental_on

execute_elemental_variables

execute_input

execute_input_on

execute_nodal_on

execute_nodal_variables

execute_on
$=$

$=$ Console

$=0$

$=0$

$=0$

$=$

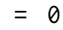

$=$

$=1$

$=$

$=$

$=1$

$=1$

$=$

$=$

1

$=$ ' INITIAL
\# Enable/disable the use of the displaced

\# mesh for outputting

\# Print detailed diagnostics on timestep

\# calculation

\# This list of output flags is added to the \# existing flags (initial|linear|nonlinear

\# timestep_end|timestep_begin|final|

\# failed|custom)

\# to execute only at that moment

\# When true the date and time are appended

\# to the output filename.

\# The format of the date/time to append,

\# if not given UTC format used (see

\# http://www.cplusplus.com/reference

\#/ctime/strftime).

\# Append '_oversample' to the output

\# file base

\# Adds user-defined labels for accessing

\# object parameters via control logic.

\# Set the enabled status of the Mooseobject.

\# Time at which this output object stop

\# operating

\# Control the output of elemental variables

\# Enable/disable the output of elemental

\# variables

\# Enable/disable the output of input file

\# information

\# Enable/disable the output of the input file

\# Control the output of nodal variables

\# Enable/disable the output of nodal

\# variables

IMESTEP_END' \# Set to

\# (none |initial|linear|nonlinear |

\# timestep_end|timestep_begin|final|

\# failed(custom)

\# to execute only at that moment

execute_postprocessors_on =

execute_scalar_variables = 1

execute_scalars_on =

execute_system_information $=1$

execute_vector_postprocessors $=1$

file

$=$

file_base

$=$

hide

$=$

interval

$=1$

linear_residual_dt_divisor

$=1000$

linear_residual_end_time

linear_residual_start_time

\# Control of when postprocessors are output

\# Enable/disable the output of aux scalar

\# variables

\# Control the output of scalar variables

\# Enable/disable the output of the simulation

\# information

\# Enable/disable the output of vector

\# postprocessors

\# The name of the mesh file to read, for

\# oversampling

\# The desired solution output name without an

\# extension

\# A list of the variables and postprocessors

\# that should NOT be output to the Exodus

\# file (may include Variables,

\# Scalarvariables, and Postprocessor names).

\# The interval at which time steps are output

\# to the solution file

\# Number of divisions applied to time step

\# when outputting linear residuals

\# Specifies an end time to begin output on

\# each linear residual evaluation

\# Specifies a start time to begin output on

\# each linear residual evaluation 


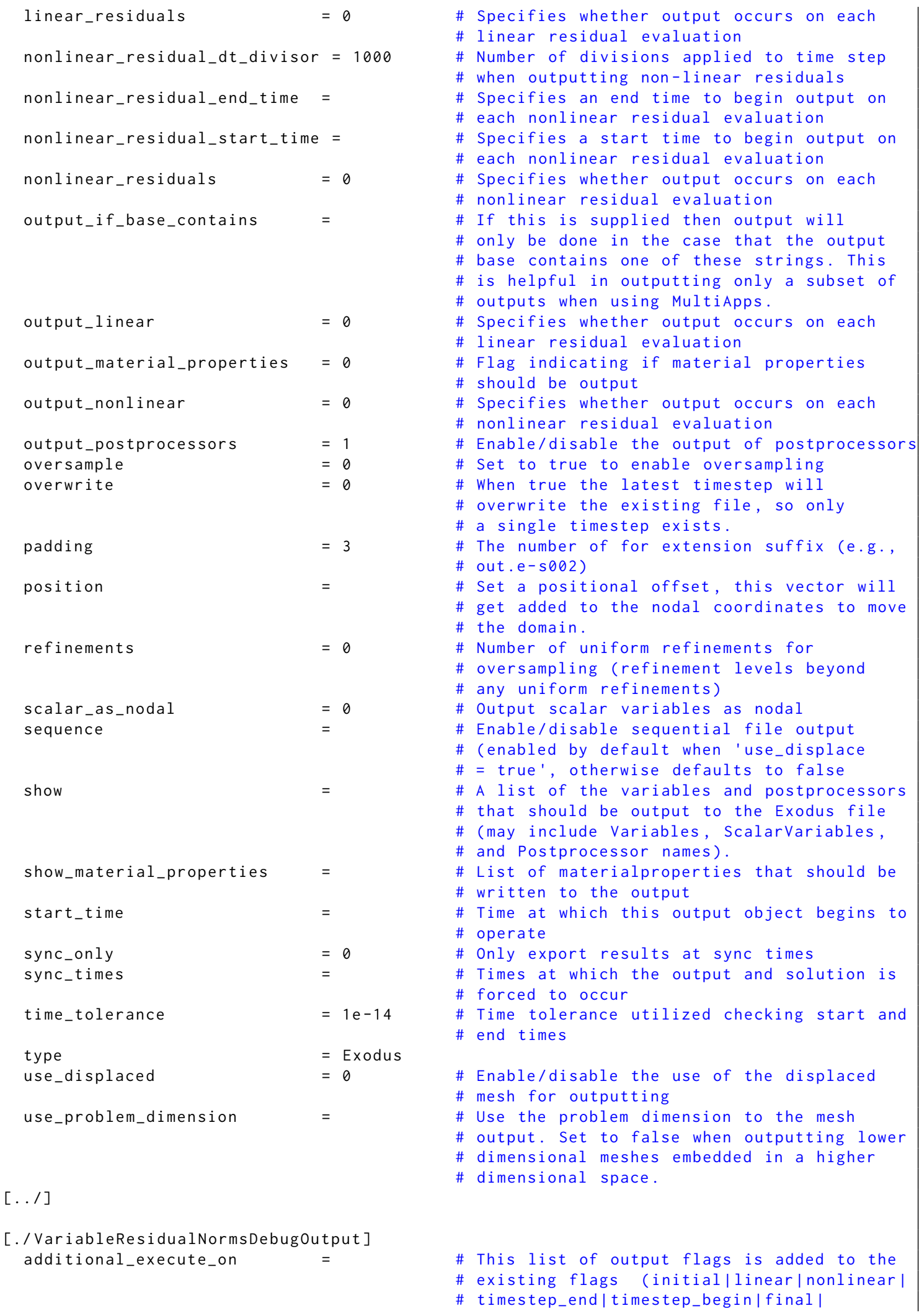




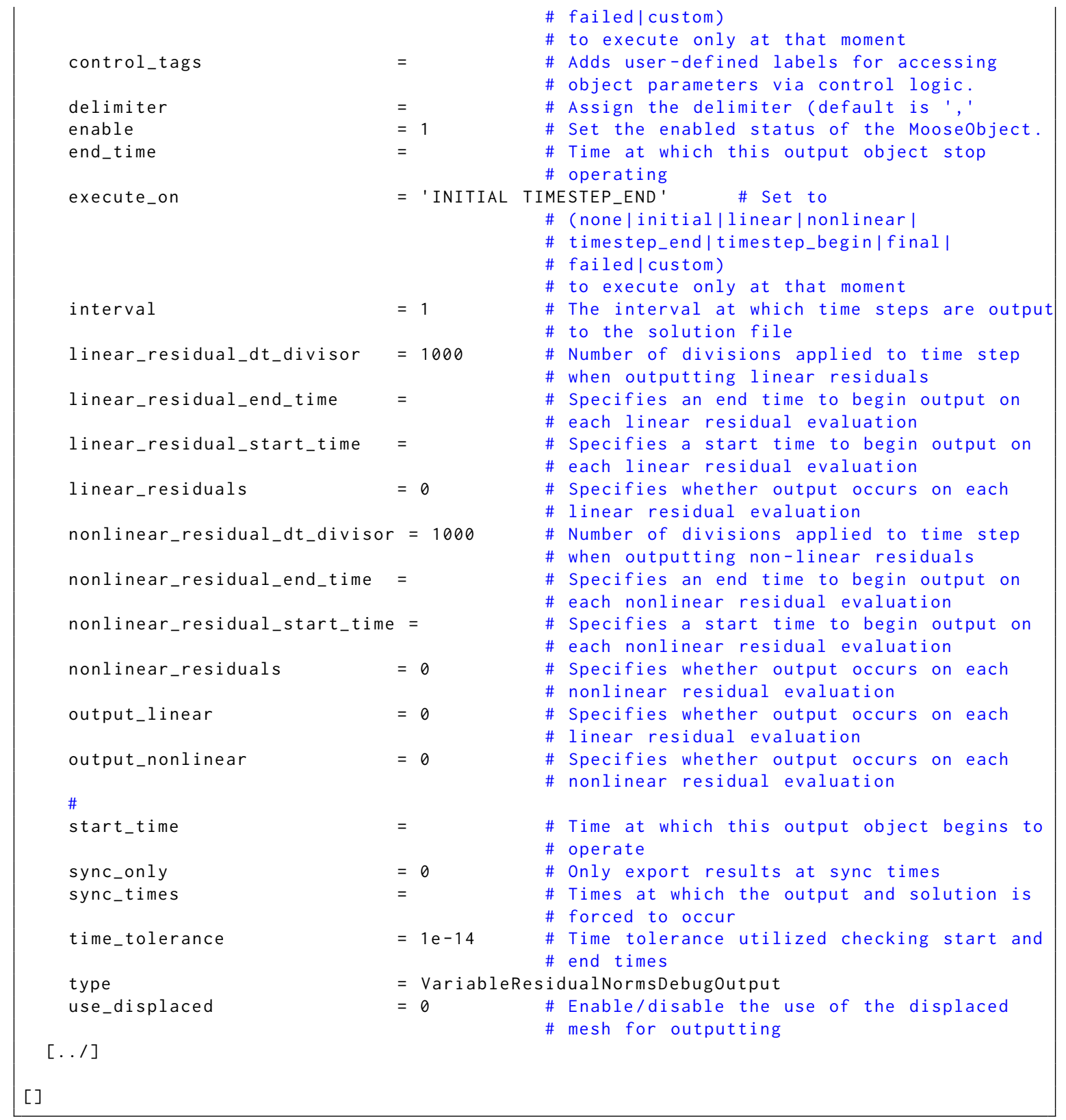

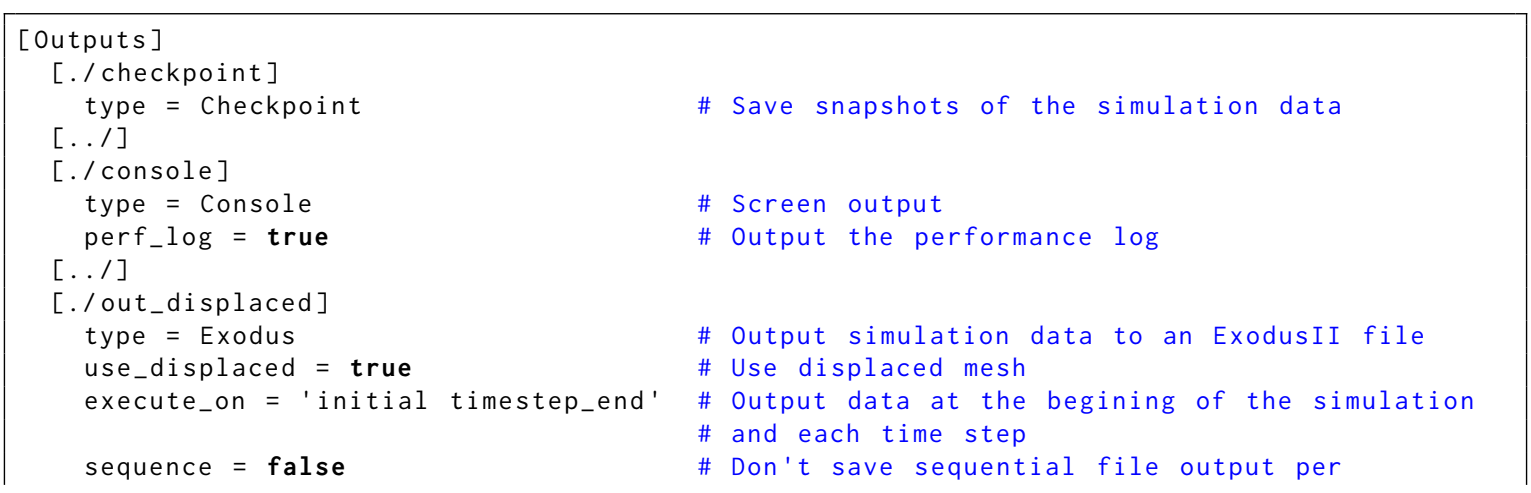


[../]

\# time step

[] 


\section{Example Problems}

In this section, several examples are given to demonstrate that how SAM is used to perform nuclear reactor safety related thermal-hydraulics analysis with input file also provided.

\subsection{Heat Conduction Problem}

The 2-D radial and axial steady-state conduction equation was solved for a generic long solid rod, as illustrated in Figure 5.1. The same case is also included in the TRACE fundamental validation cases [16]. The heat structure has a length of $20 \mathrm{~cm}$ and radius of $5 \mathrm{~mm}$. It has a uniform heat source of $1000 \mathrm{~W}$ distributed within the rod, and constant thermal conductivity of $2 \mathrm{~W} / \mathrm{mK}$. The solid rod is immersed in a pool of water having a constant temperature of $300 \mathrm{~K}$ in the bottom 10 $\mathrm{cm}$ and $500 \mathrm{~K}$ in the top $10 \mathrm{~cm}$. A constant heat transfer coefficient of $1000 \mathrm{~W} / \mathrm{m}^{2} \mathrm{~K}$ is applied to the outer surface of the rod. The tabulated analytical solution values from Table A.1.2 of Reference [16] are used here in Table 5.1 for comparison to the temperatures calculated by SAM.

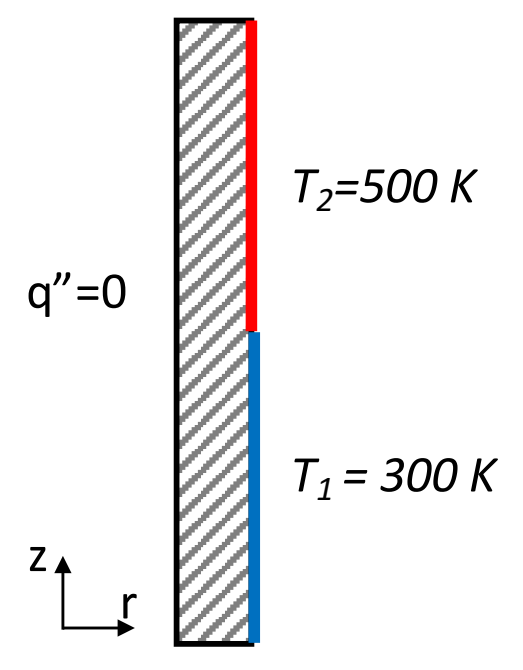

Figure 5.1: SAM model of the 2-D heat conduction problem. 
Table 5.1: Comparison of SAM and Analytical Solutions for the Steady State Axial-Radial Heat Conduction Problem

\begin{tabular}{|c|c|c|c|}
\hline Location (m) & Analytical (K) & SAM (K) & Error $(\mathbf{K})$ \\
\hline 0 & 658.1 & 658.1 & 0.0 \\
\hline 0.01 & 658.1 & 658.1 & 0.0 \\
\hline 0.02 & 658.1 & 658.1 & 0.0 \\
\hline 0.03 & 658.1 & 658.1 & 0.0 \\
\hline 0.04 & 658.1 & 658.1 & 0.0 \\
\hline 0.05 & 658.1 & 658.1 & 0.0 \\
\hline 0.06 & 658.1 & 658.1 & 0.0 \\
\hline 0.07 & 658.1 & 658.1 & 0.0 \\
\hline 0.08 & 658.2 & 658.2 & 0.0 \\
\hline 0.09 & 662.7 & 662.3 & -0.4 \\
\hline 0.1 & 758.1 & 758.1 & 0.0 \\
\hline 0.11 & 853.9 & 853.9 & 0.0 \\
\hline 0.12 & 857.9 & 858.0 & 0.1 \\
\hline 0.13 & 858.1 & 858.1 & 0.0 \\
\hline 0.14 & 858.1 & 858.1 & 0.0 \\
\hline 0.15 & 858.1 & 858.1 & 0.0 \\
\hline 0.16 & 858.1 & 858.1 & 0.0 \\
\hline 0.17 & 858.1 & 858.1 & 0.0 \\
\hline 0.18 & 858.1 & 858.1 & 0.0 \\
\hline 0.19 & 858.1 & 858.1 & 0.0 \\
\hline
\end{tabular}

The SAM simulation was run with Steady solver for this test case. The calculated steady-state conditions and analytical solution of centerline temperature distributions are compared in Table 5.1 and shown in Figure 5.2. The results given in Table 5.1 demonstrate that the SAM solutions of the 2-D heat-conduction equation are accurate. The largest errors are where temperature profile is steepening. Note that a relative coarse mesh, 40 (20-axial and 2-radial) elements total, was used in SAM simulations. The errors can be reduced if a finer mesh is used. 


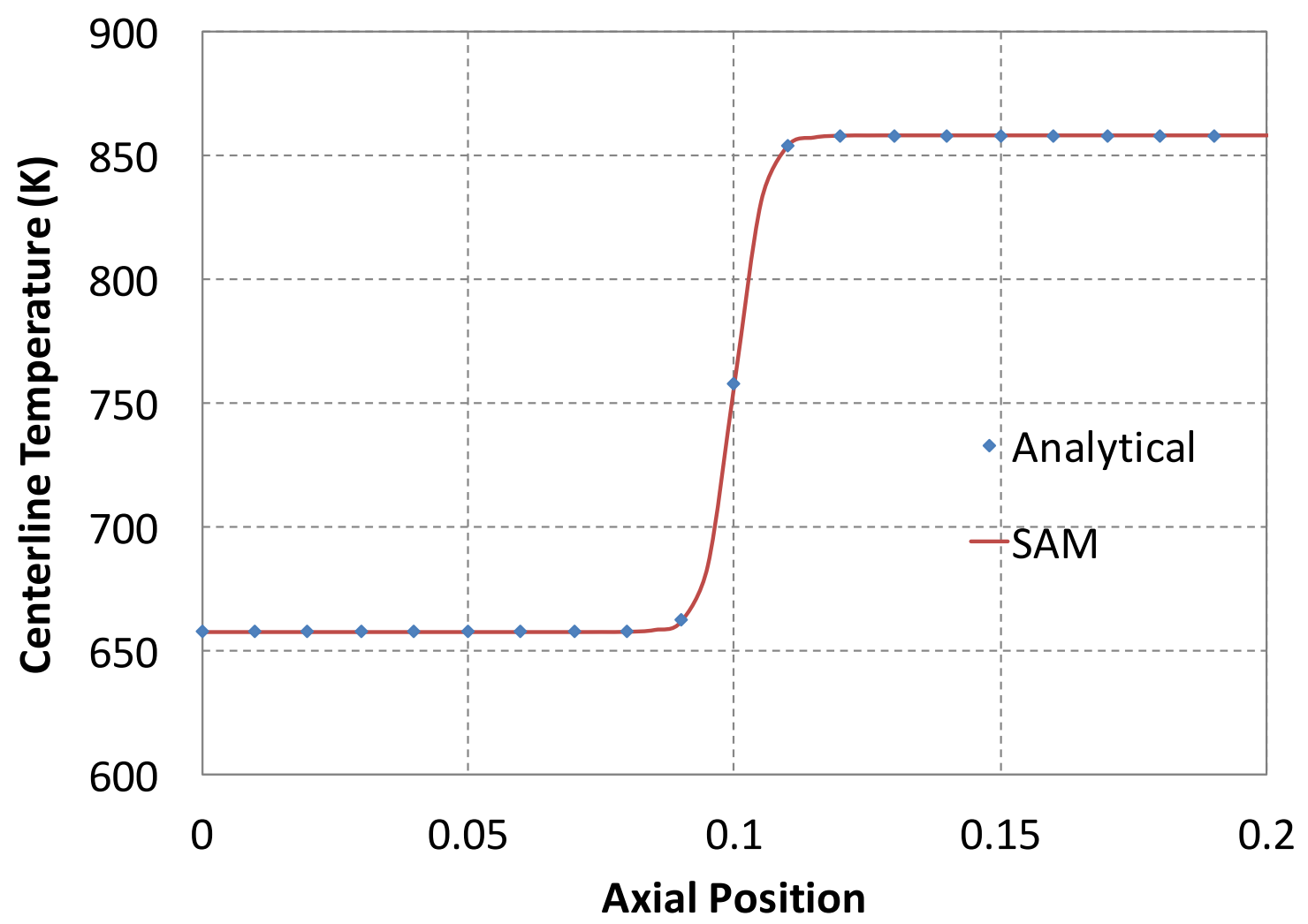

Figure 5.2: Comparisons of centerline temperature distributions of the heated rod, 2D conduction.

The input file of this example problem is shown as follows:

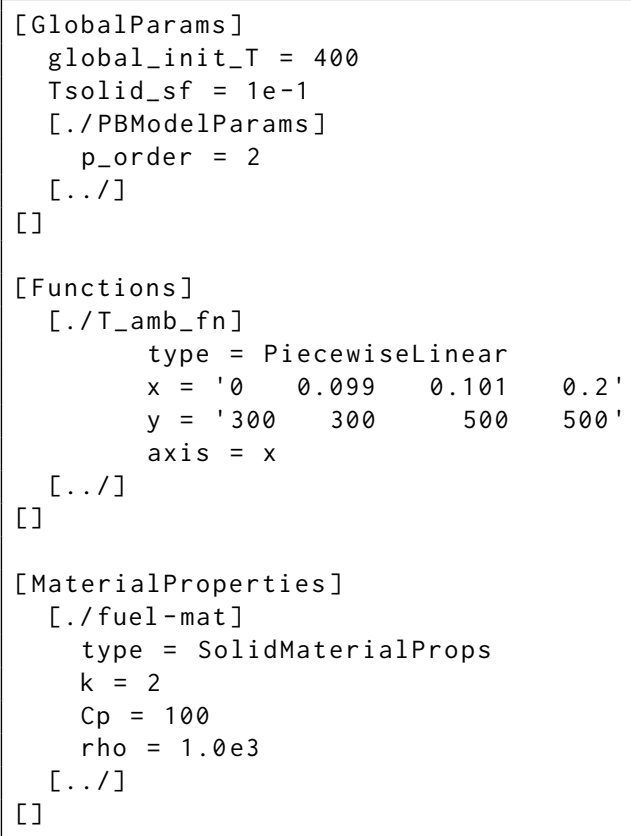




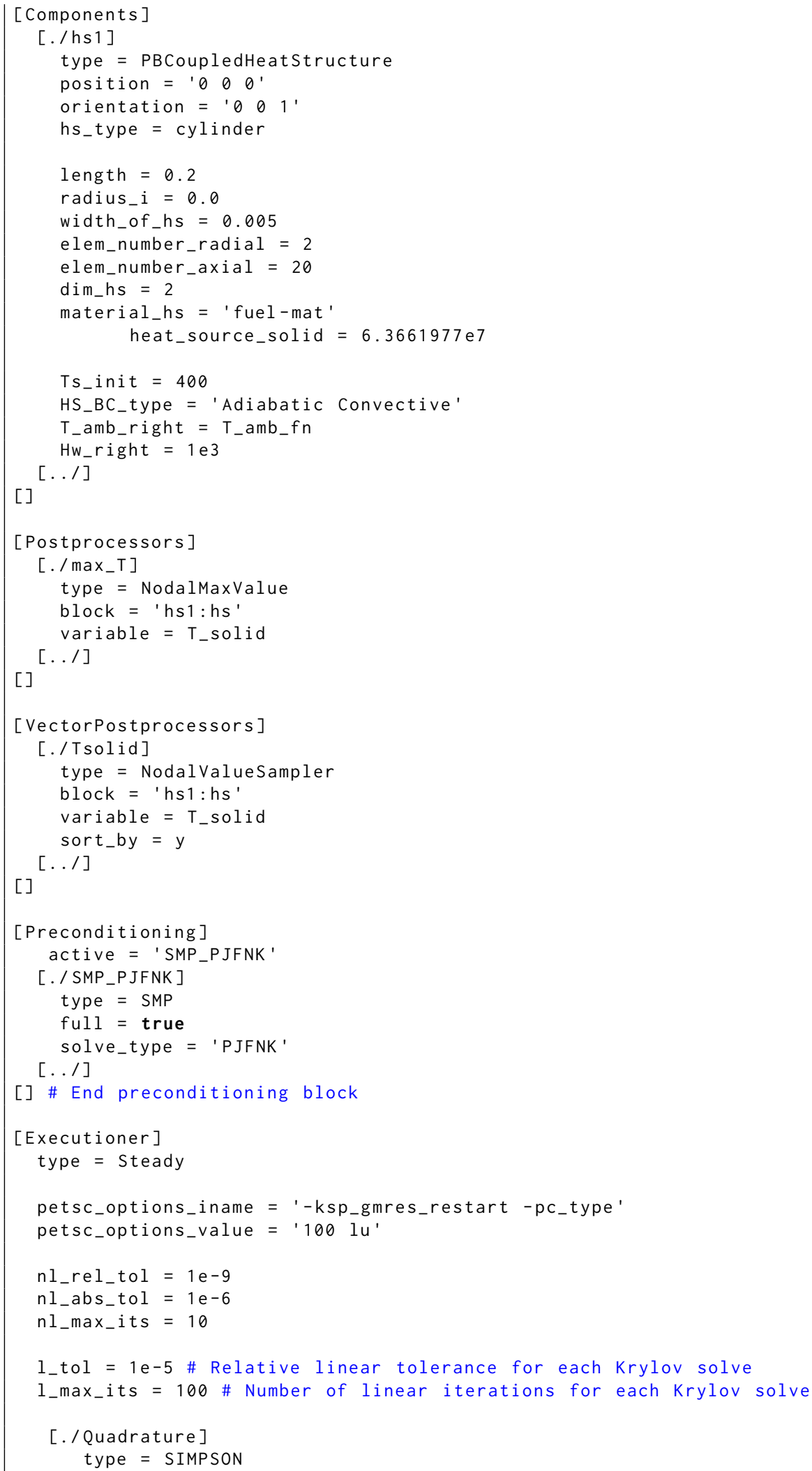


[../]

[] \# close Executioner section

[Outputs]

perf_graph = true

print_linear_residuals = false

[./out_displaced]

type = Exodus

use_displaced = true

execute_on = 'initial timestep_end'

sequence $=$ false

$[\ldots /]$

$[. / \mathrm{csv}]$

type $=$ CSV

$[\ldots /]$

[./ console]

type $=$ Console

$[\ldots /]$

[]

112 


\subsection{Single Channel Flow}

A simple pipe flow problem is presented here, with fixed constant or time-varying boundary conditions. The inlet temperature of the one-meter pipe is fixed at $628 \mathrm{~K}$, or oscillates following a sinusoidal distribution, $T_{i n}(t)=628+100 \sin (\pi t)$; the inlet velocity is fixed, $u_{i n}(t)=0.5 \mathrm{~m} / \mathrm{s}$; and the initial pipe temperate is at $628 \mathrm{~K}$. After executing the test problems, the results can be imported into Paraview for visualization, as shown in Figure 5.3. The transient responses of the inlet temperature wave propagation problem are shown in Figure 5.4, where the code predictions agreed very well with the analytical solutions. This is because of the high-order accuracy in both spatial and temporal (BDF2) discretizations used in SAM.

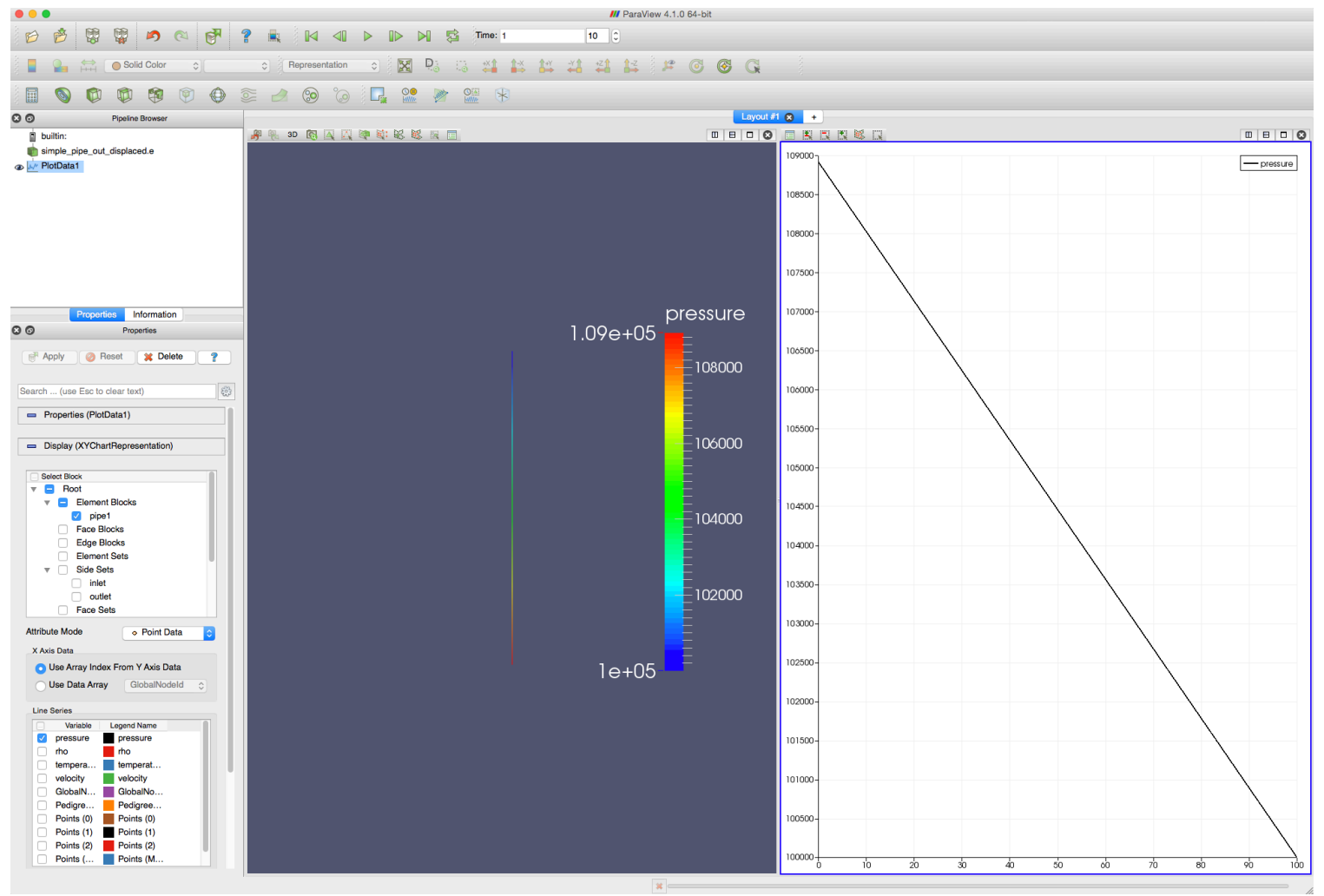

Figure 5.3: Example of SAM results shown in Paraview.

The input file of this example problem is shown as follows:

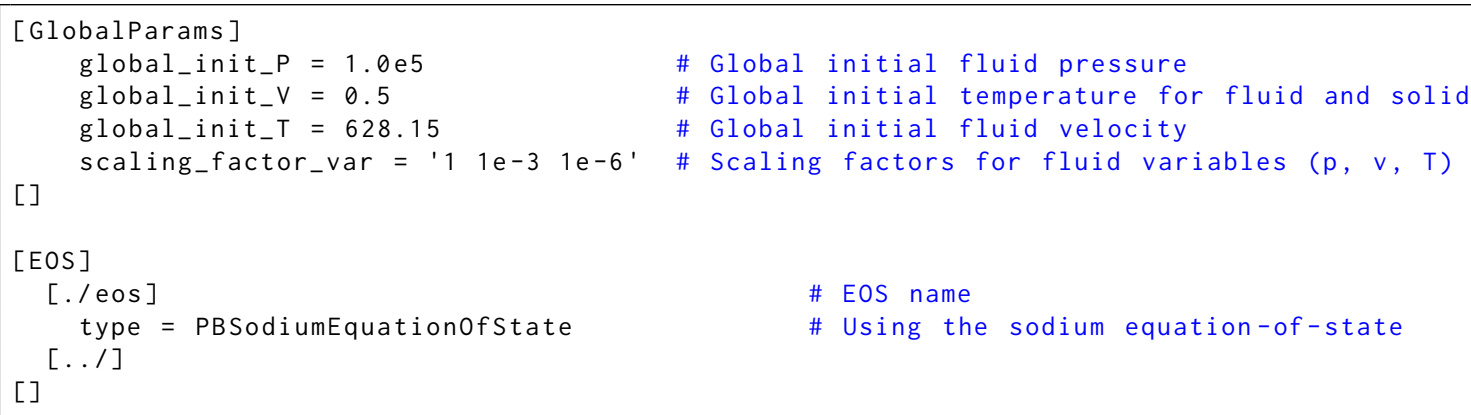




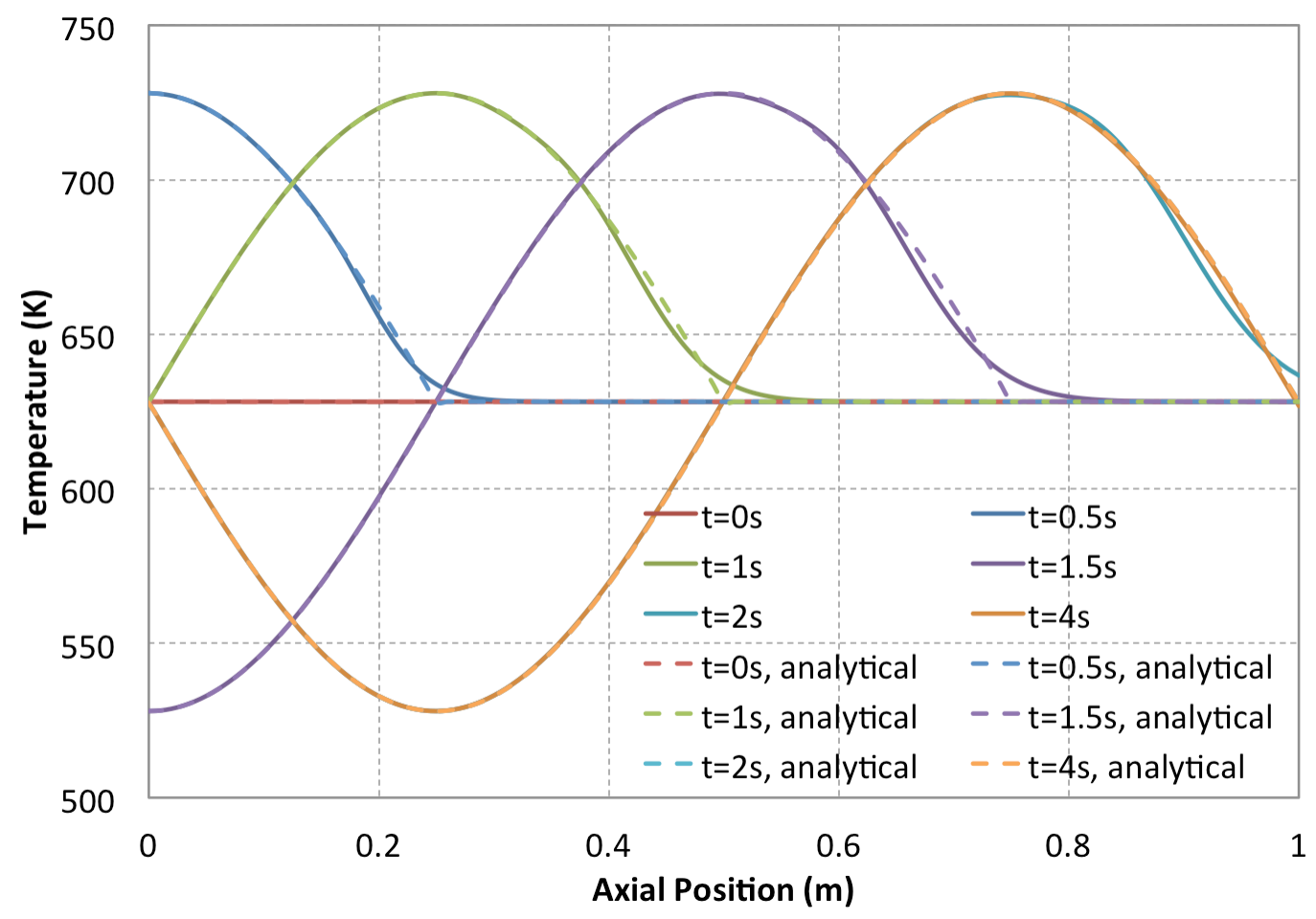

Figure 5.4: Transient responses of the pipe under inlet temperature oscillation, BDF2.

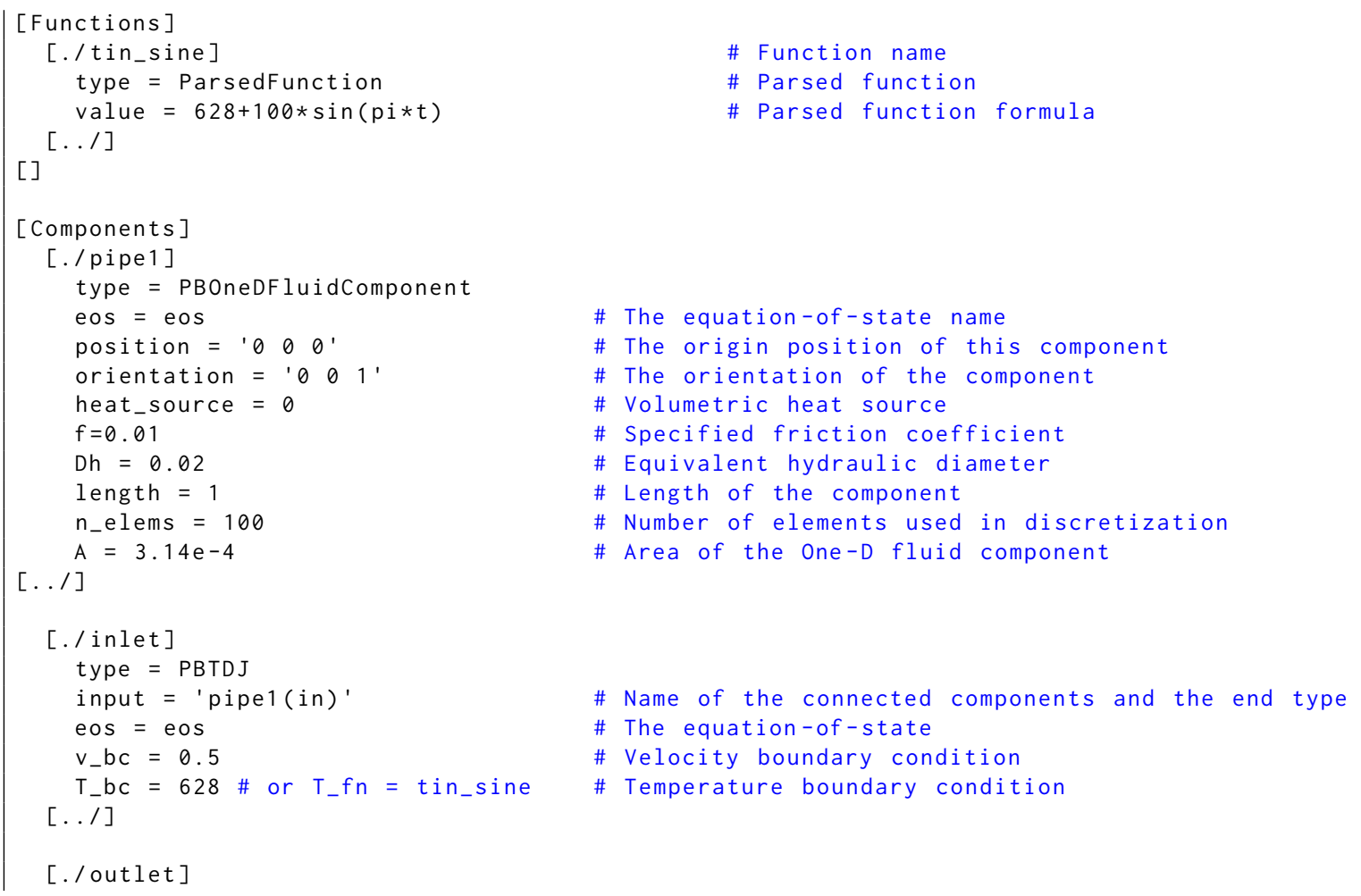




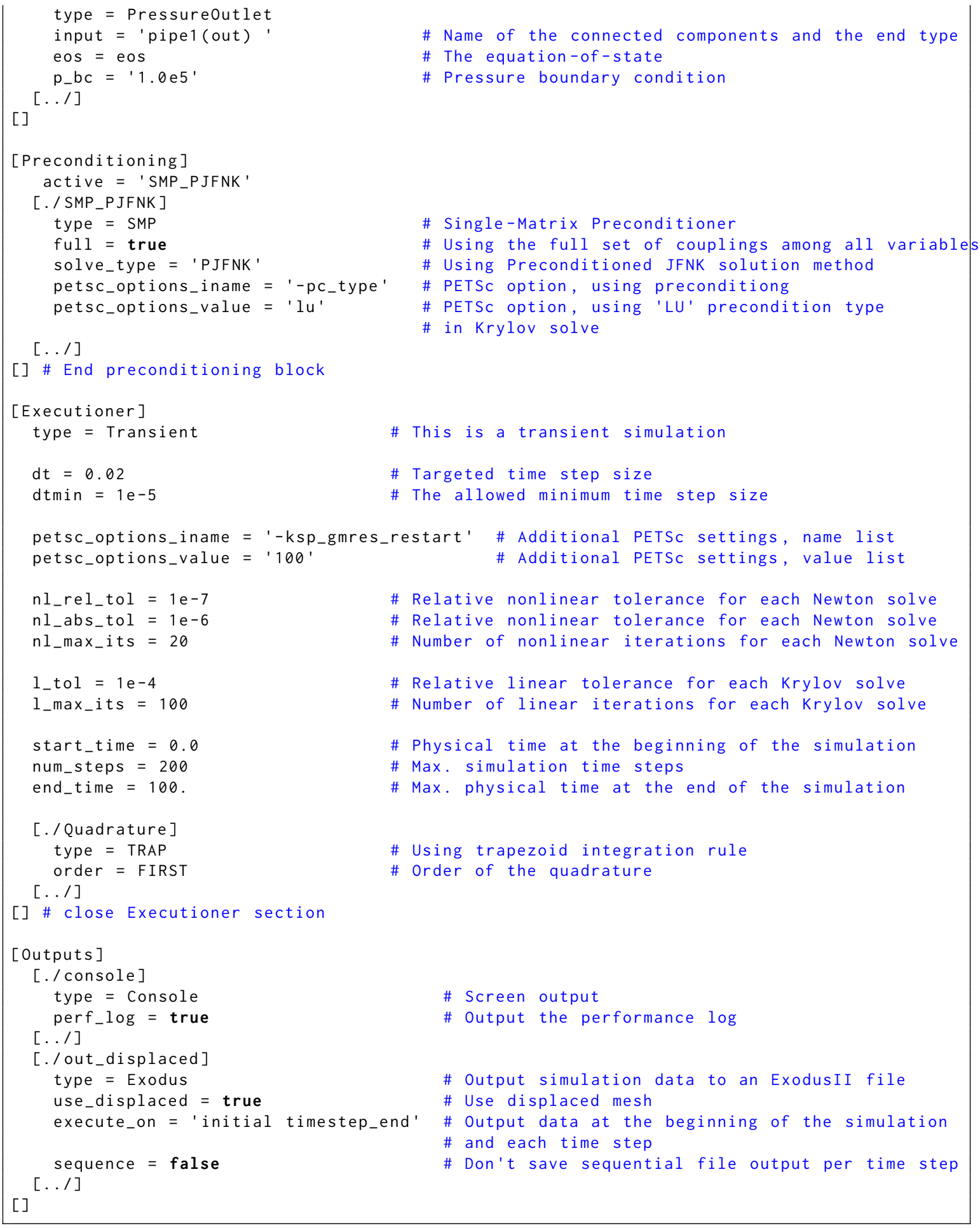




\subsection{Core Channel}

A simple core channel problem (coolant flow and solid conduction in fuel assembly) is presented here with uniform power distribution inside the fuel pin. The schematic of the spatial discretization of the core channel problem is shown in Figure 5.5. The different lines of colors on the left represent different heat structures in an SFR fuel pin (i.e., fuel, sodium gap, and clad). The inlet of the core channel flow is fixed at constant temperature and flow rate. Constant material thermo-physical properties are assumed for this test. Therefore, the analytical solutions of this test problem can be easily derived, with coolant temperature:

$$
T_{\text {coolant }}(z)=T_{\text {in }}+\frac{q^{\prime}}{\dot{m} c_{p}} z
$$

and the fuel centerline temperature:

$$
T_{f, c l}(z)=T_{i n}+q^{\prime}\left[\frac{z}{\dot{m} c_{p}}+\frac{1}{2 \pi R_{c o} h_{c}}+\frac{1}{2 \pi k_{c}} \ln \left(\frac{R_{c o}}{R_{c i}}\right)+\frac{1}{2 \pi R_{f} h_{g}}+\frac{1}{4 \pi k_{f}}\right]
$$

The simulation results can be compared with the analytical solutions as an verification study.

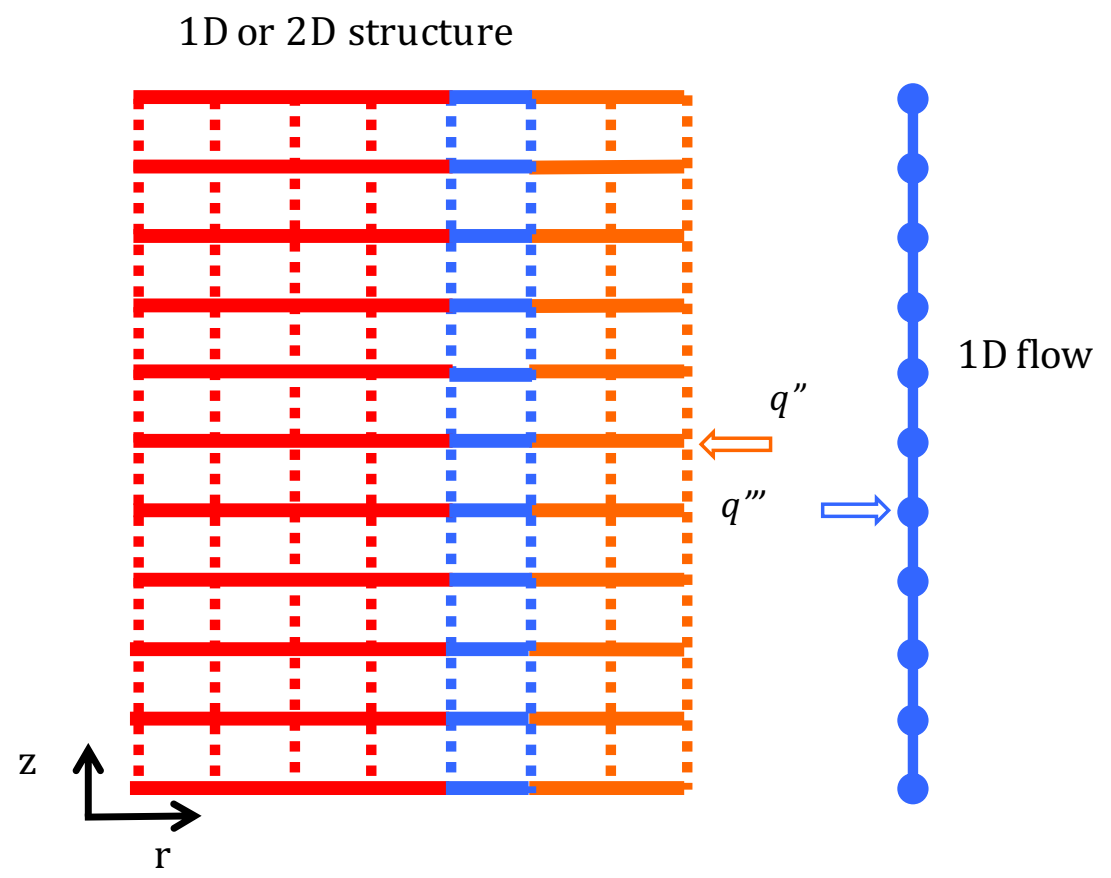

Figure 5.5: The schematic of the spatial discretization of the core channel problem.

The input file of this example problem is shown as follows:

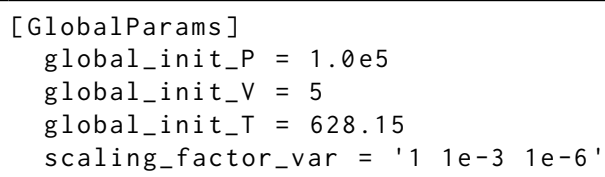




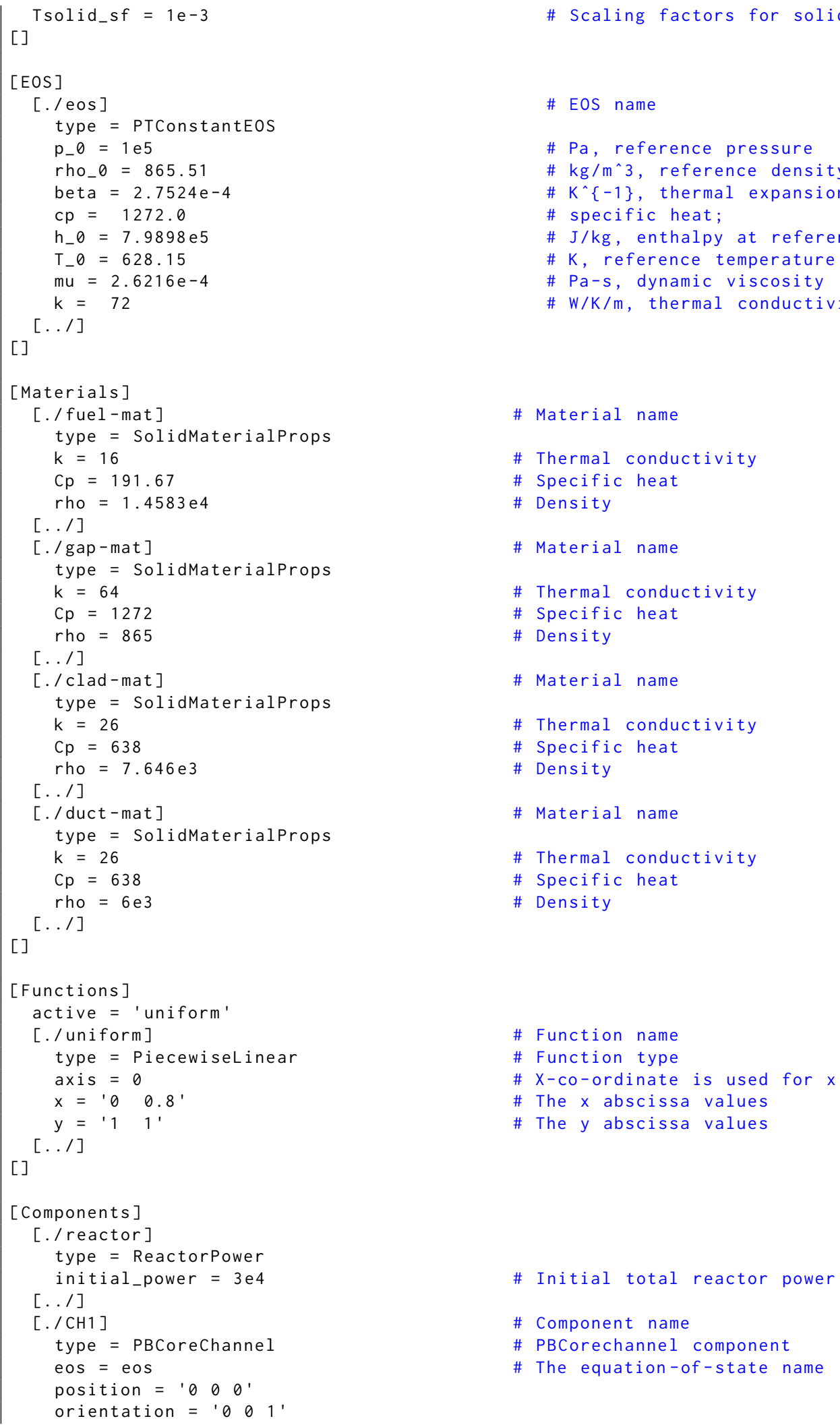




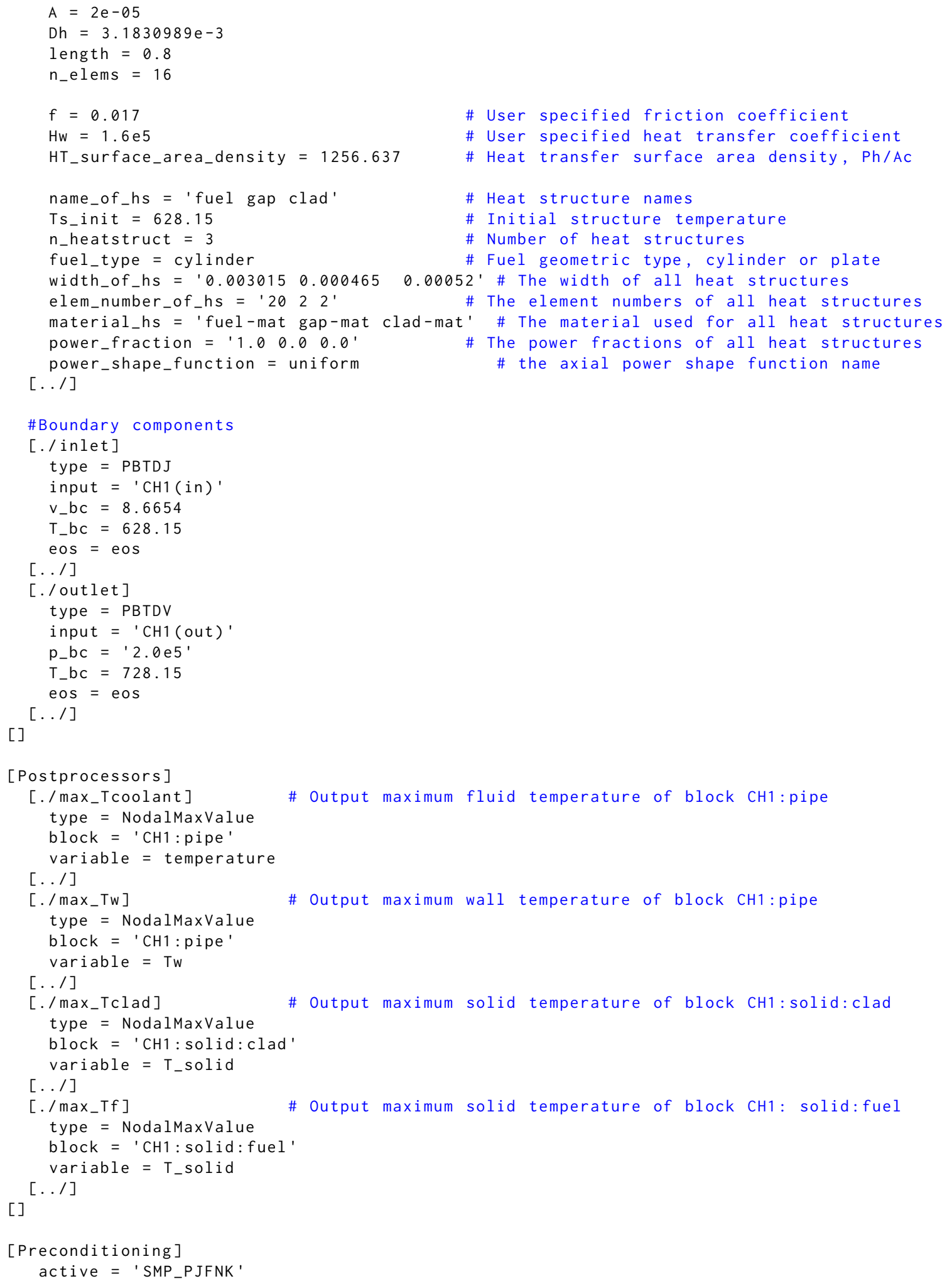




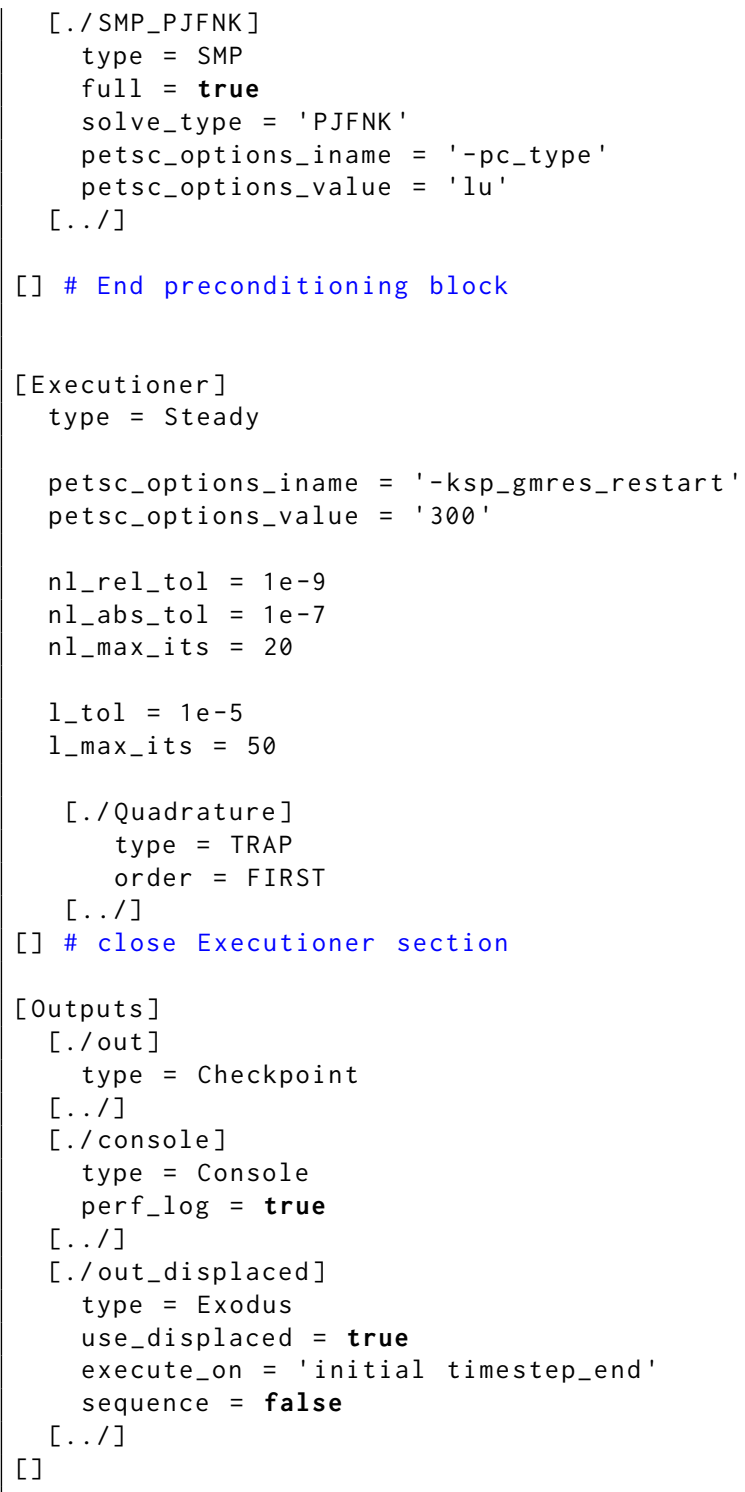




\subsection{Heat Exchanger}

An example of a counter-current heat exchanger problem is presented here. The inlet temperatures are $783 \mathrm{~K}$ and $606 \mathrm{~K}$ for the primary and secondary pipes. The mass flow rates are also fixed at the inlets of the two sides. Because the flow rates are very close for the two sides, linear temperature distributions are expected for the two sides, as the code predictions shown in Figure 5.6.

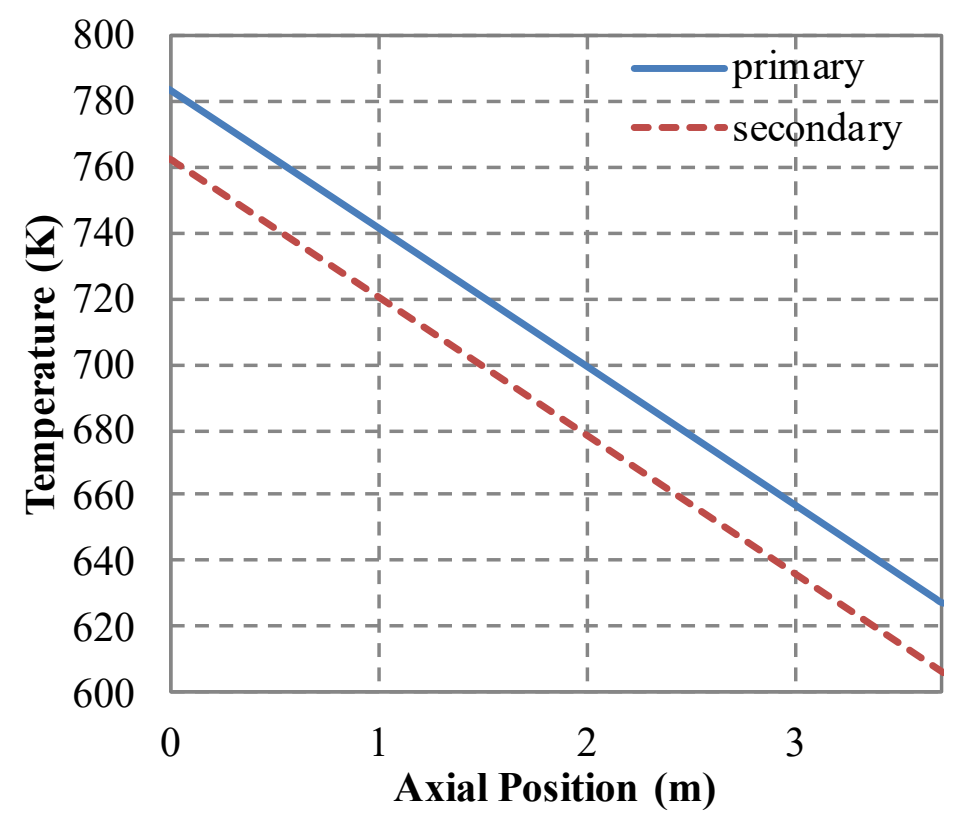

Figure 5.6: Temperature distribution of a counter-current heat exchanger.

The input file of this example problem is shown as follows:

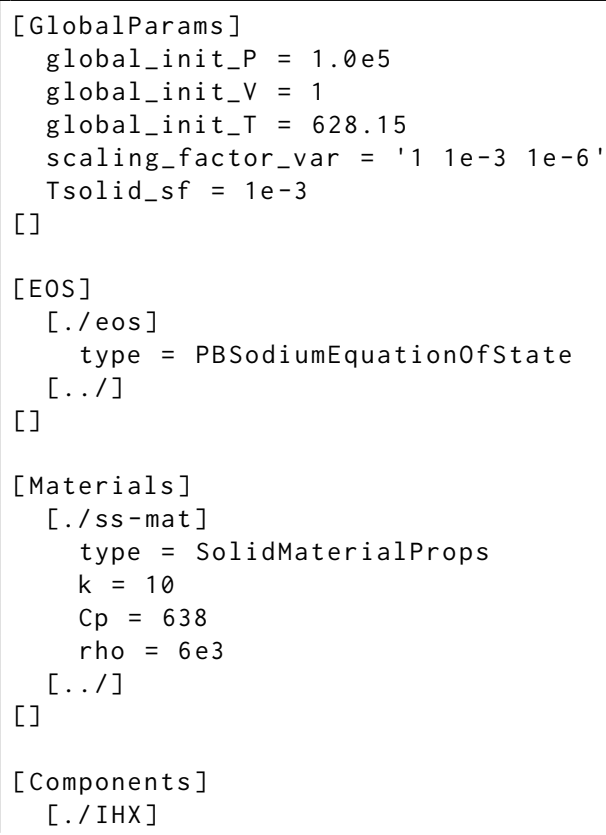




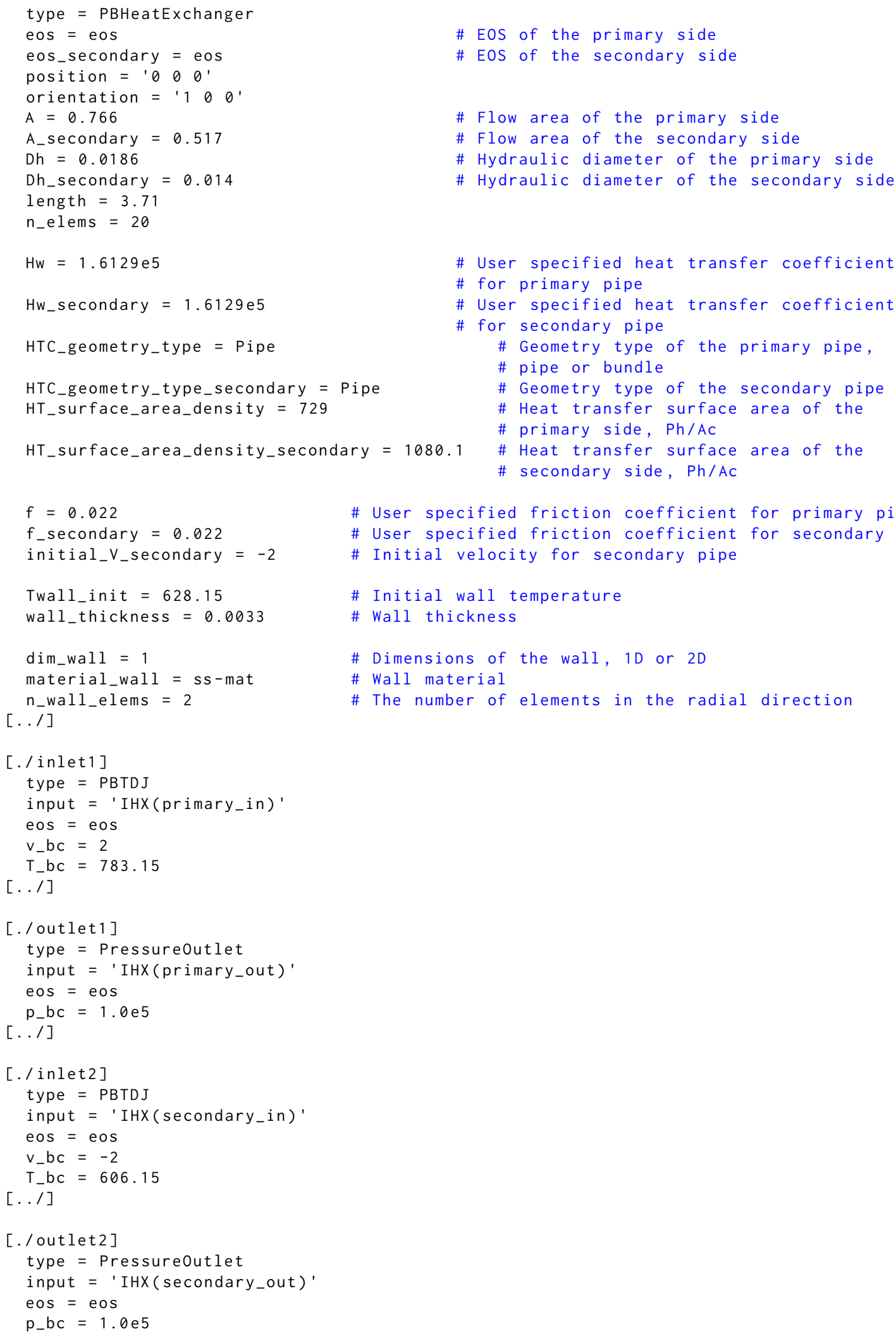




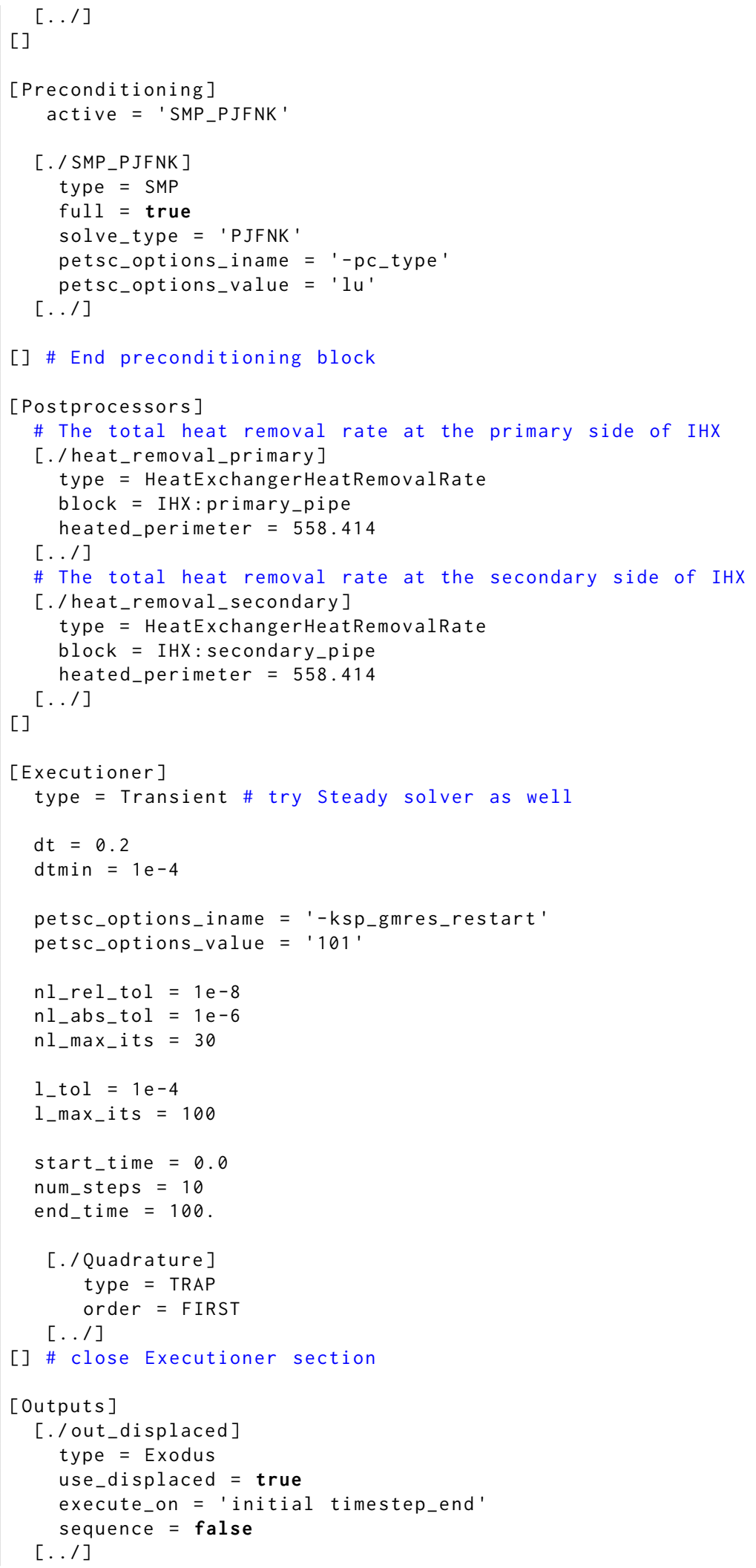


[./ console]

type $=$ Console

perf_log = true [] 


\subsection{Volume Branch}

An example problem with a VolumeBranch component included is presented here. The boundary and the initial conditions of the five pipes are shown in Figure 5.7 and Figure 5.8. Note that very different inlet orifice coefficients have been used for the connecting nodes. The volume of the PBVolumeBranch is $0.0314 \mathrm{~m}^{3}$, and the initial temperature of the volume is at $628.15 \mathrm{~K}$. Because Pipe 3 has very high inlet flow rate but low inlet temperature, the temperature at the VolumeBranch, outlet of Pipes 4 and 5 will decrease correspondingly, as shown in Figure 5.9.

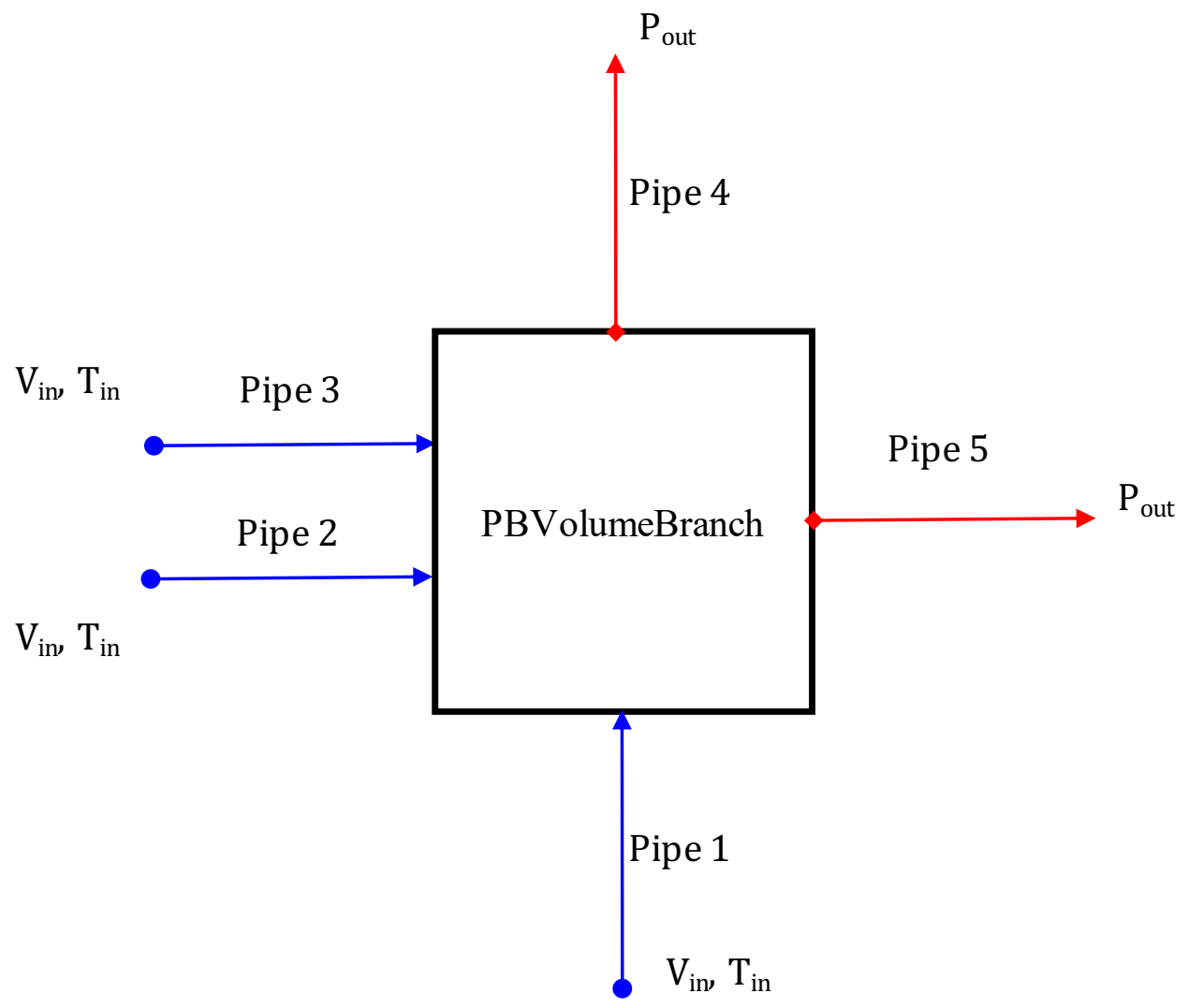

Figure 5.7: The three-pipe-in and two-pipe-out VolumeBranch test model. 


\begin{tabular}{|c|c|c|c|c|c|}
\hline Component & Pipe 1 & Pipe 2 & Pipe 3 & Pipe 4 & Pipe 5 \\
\hline Length $(\mathrm{m})$ & 1 & 1 & 1 & 1 & 1 \\
\hline Diameter (m) & 0.02 & 0.02 & 0.02 & 0.02 & 0.02 \\
\hline Boundary Type & Flow inlet & Flow inlet & Flow inlet & $\begin{array}{l}\text { Pressure } \\
\text { outlet }\end{array}$ & Pressure outlet \\
\hline $\begin{array}{l}\text { Orifice Coeff. to } \\
\text { VolumeBranch }\end{array}$ & 0.01 & 0.01 & 0.01 & 0.01 & 100 \\
\hline $\begin{array}{l}\text { Z-coordinate of the node } \\
\text { connected to } \\
\text { VolumeBranch (m) }\end{array}$ & 0 & 0.25 & 0.75 & 1 & 0.5 \\
\hline Boundary Conditions & $\begin{array}{l}\operatorname{Vin}=1 \mathrm{~m} / \mathrm{s} \\
\operatorname{Tin}= \\
628.15 \mathrm{~K}\end{array}$ & $\begin{array}{l}\operatorname{Vin}=1 \mathrm{~m} / \mathrm{s} \\
\operatorname{Tin}= \\
628.15 \mathrm{~K}\end{array}$ & $\begin{array}{l}\operatorname{Vin}=10 \mathrm{~m} / \mathrm{s} \\
\operatorname{Tin}= \\
528.15 \mathrm{~K}\end{array}$ & $\begin{array}{l}\text { Pout }=10^{5} \mathrm{~Pa} \\
\text { Tout }= \\
628.15 \mathrm{~K}\end{array}$ & $\begin{array}{l}\text { Pout }=1.5 \times 10^{5} \\
\text { Pa } \\
\text { Tout }=628.15 \mathrm{~K}\end{array}$ \\
\hline \multicolumn{6}{|l|}{ Initial Conditions } \\
\hline Pressure $(\mathrm{Pa})$ & \multicolumn{5}{|c|}{$1.5 \times 10^{5}$} \\
\hline Velocity $(\mathrm{m} / \mathrm{s})$ & \multicolumn{5}{|c|}{1} \\
\hline Temperature (K) & \multicolumn{5}{|c|}{628.15} \\
\hline
\end{tabular}

Figure 5.8: Input parameters of the three pipe in and two pipe out VolumeBranch test model.

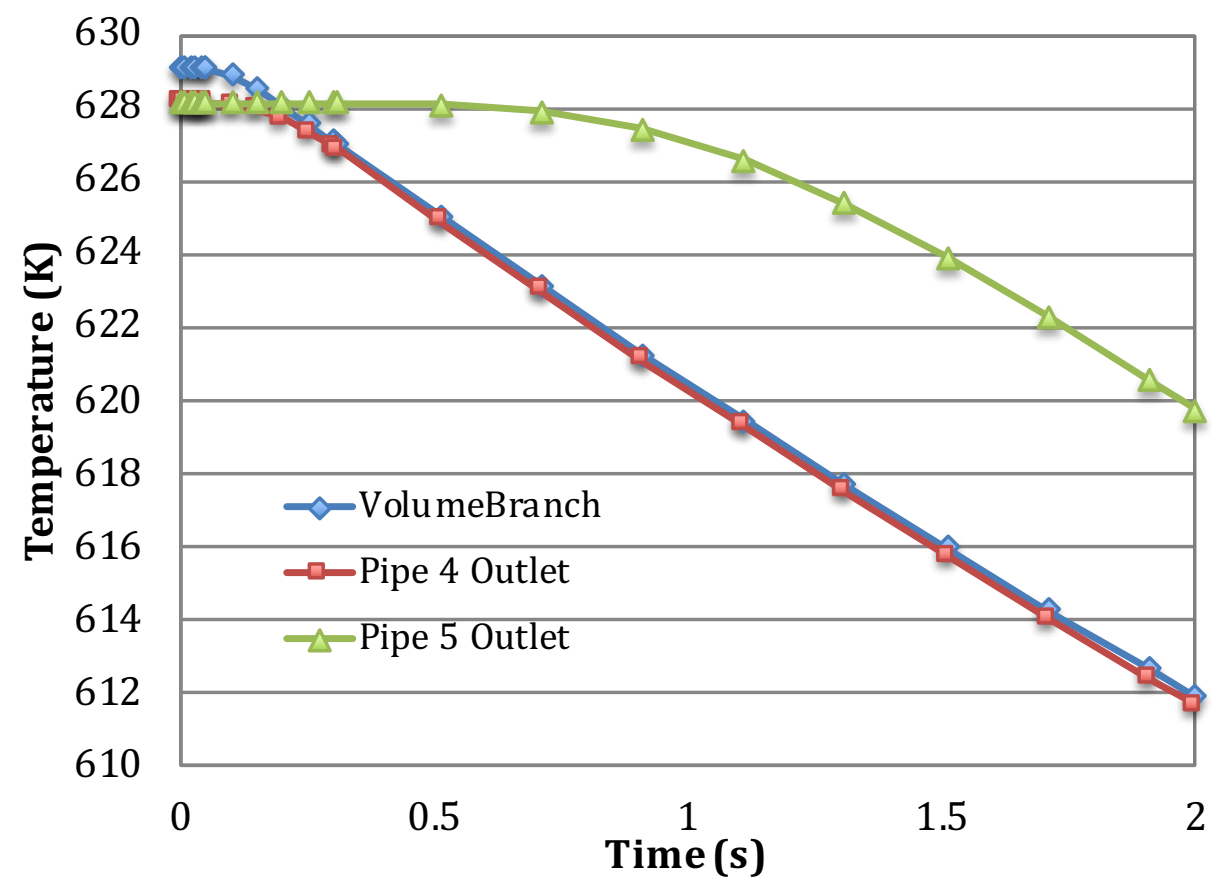

Figure 5.9: Transient temperature response at the VolumeBranch and pipe outlets.

The input file of this example problem is shown as follows:

[GlobalParams] 


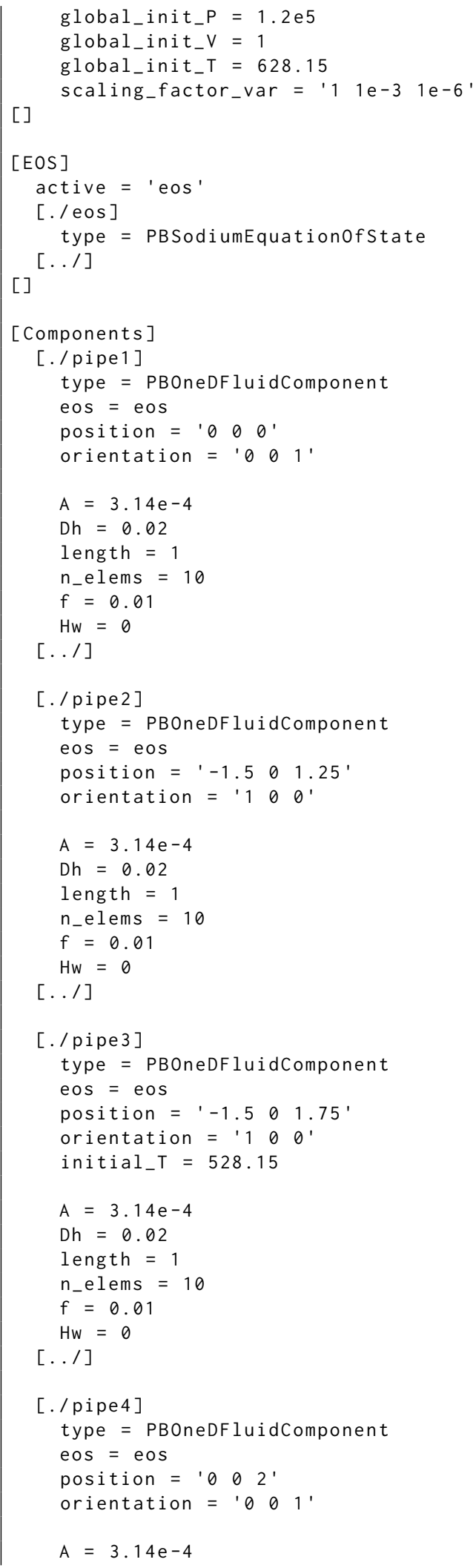




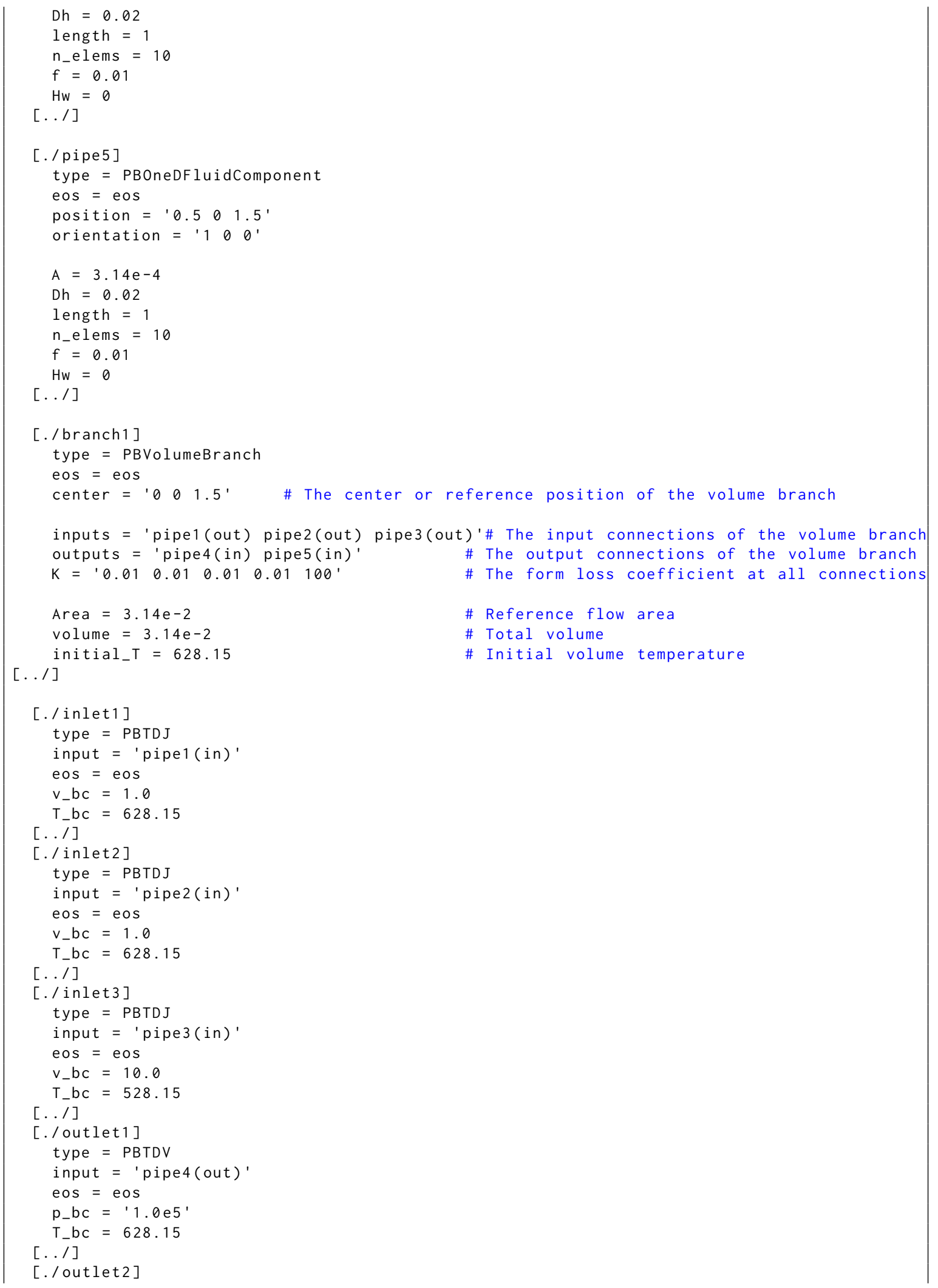




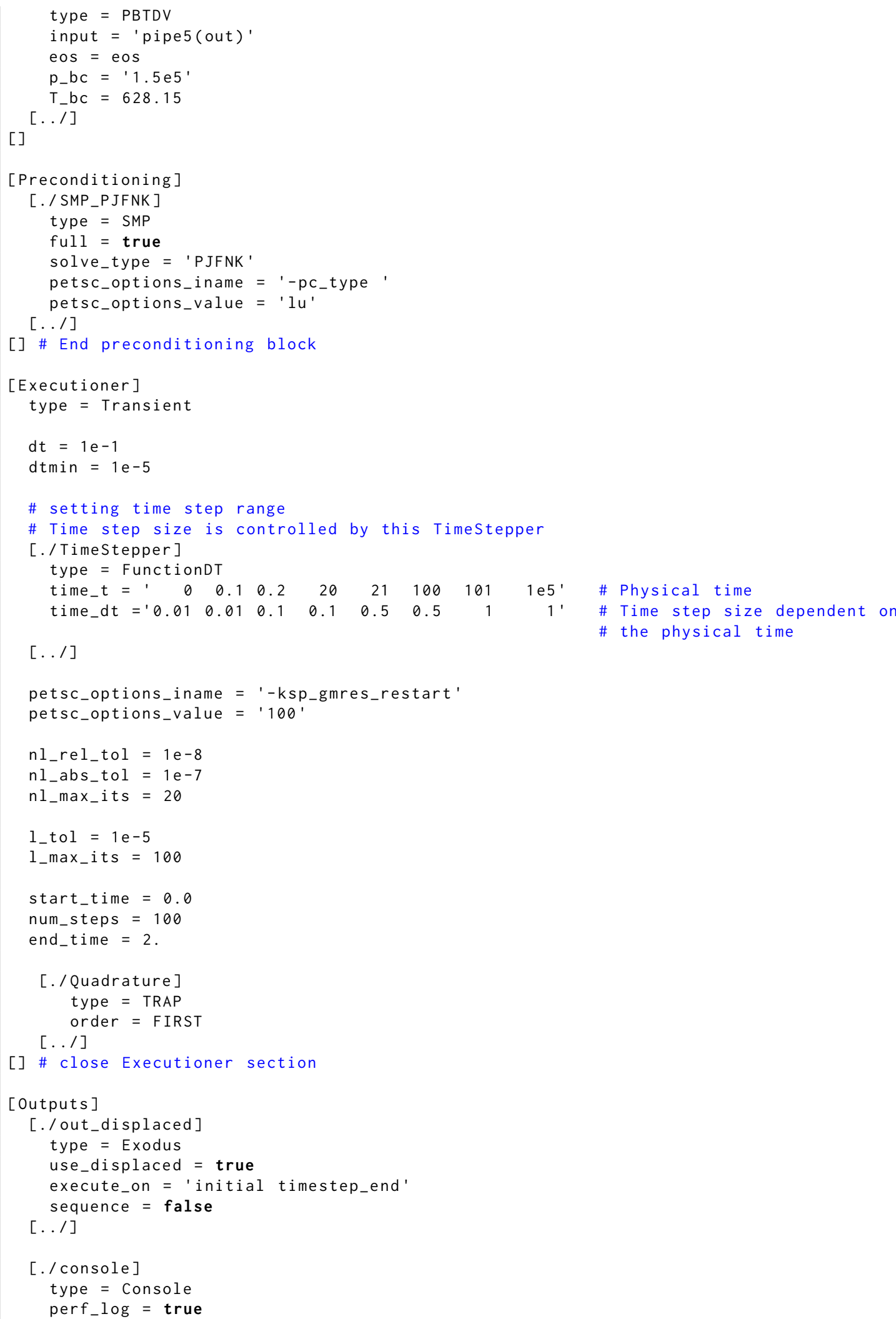


[../]

[] 


\subsection{A Simple Loop Model}

An example problem with a simple loop problem is presented here. It consists of six 1-D pipes (PBOneDFluidComponent) and a heat exchanger (PBHeatExchanger). One pipe is internally heated, as shown in Figure 5.10. The primary loop (including the heat exchanger) is connected by a set of PBSingleJunctions, a PBBranch, and a Pump. The secondary side of the heat exchanger has fixed inlet velocity and temperature and fixed outlet pressure boundary conditions. Note that if the Pump is replaced by a PBBranch, the loop will be derived by natural circulation.

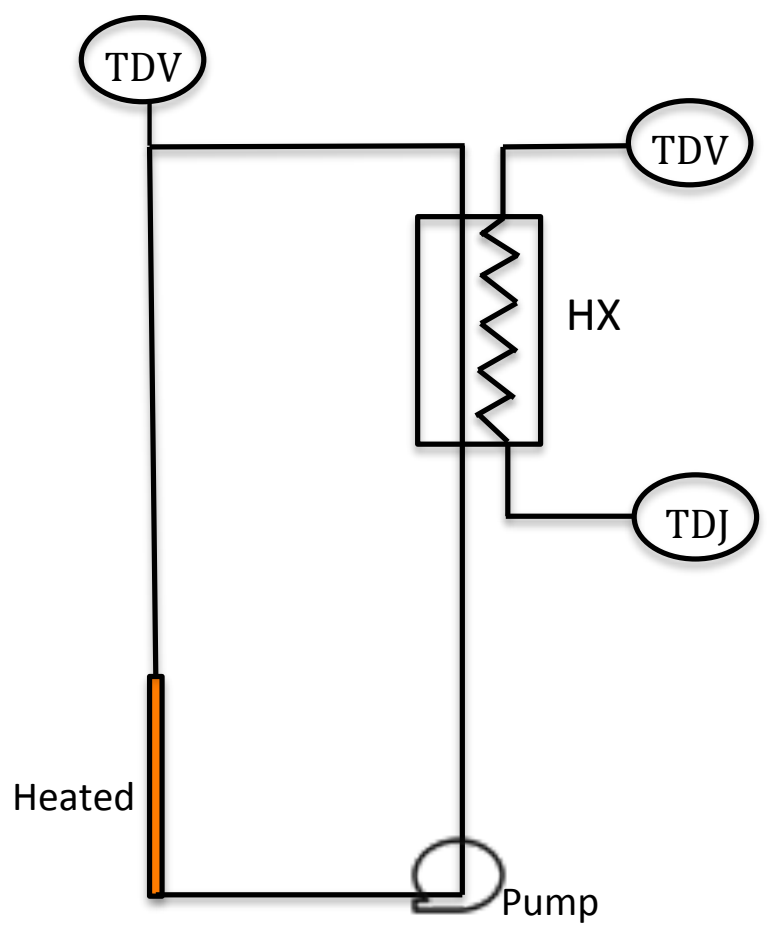

Figure 5.10: Schematics of the a test loop problem.

The input file of this example problem is shown as follows:

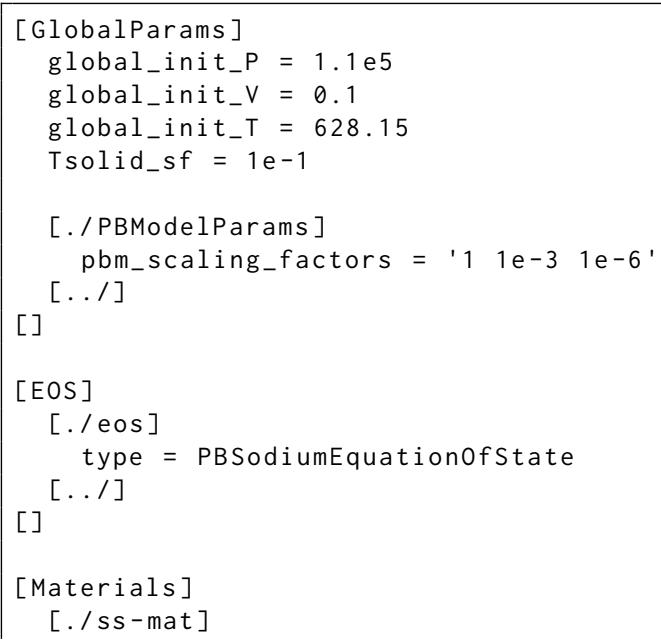




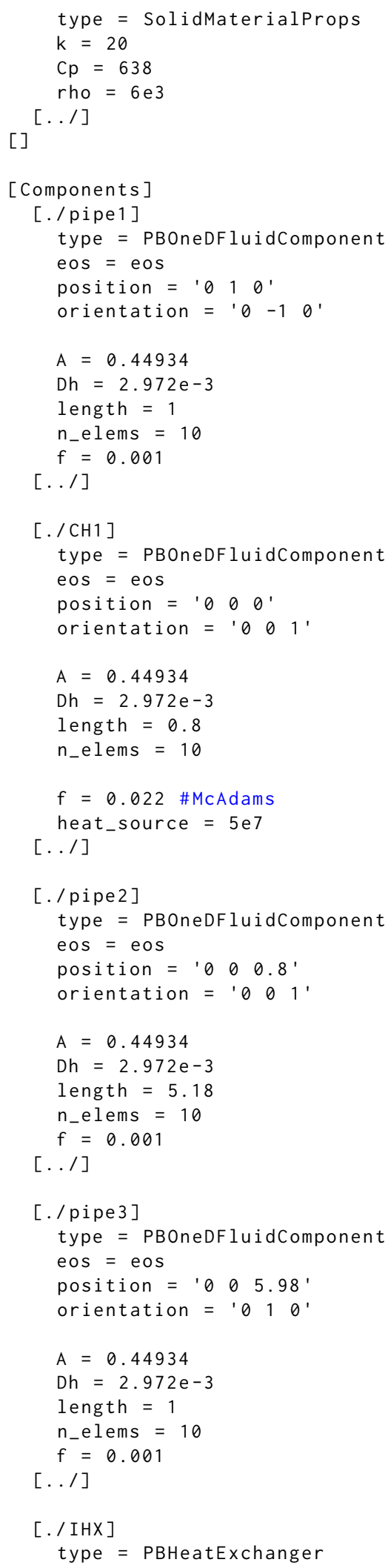




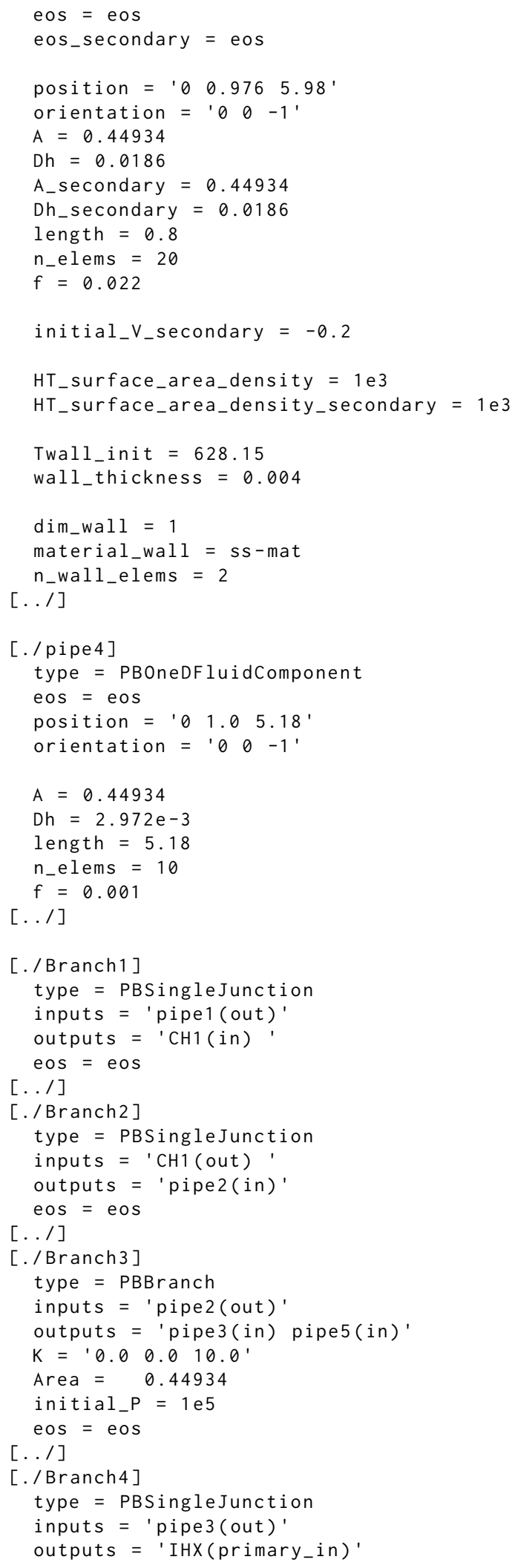




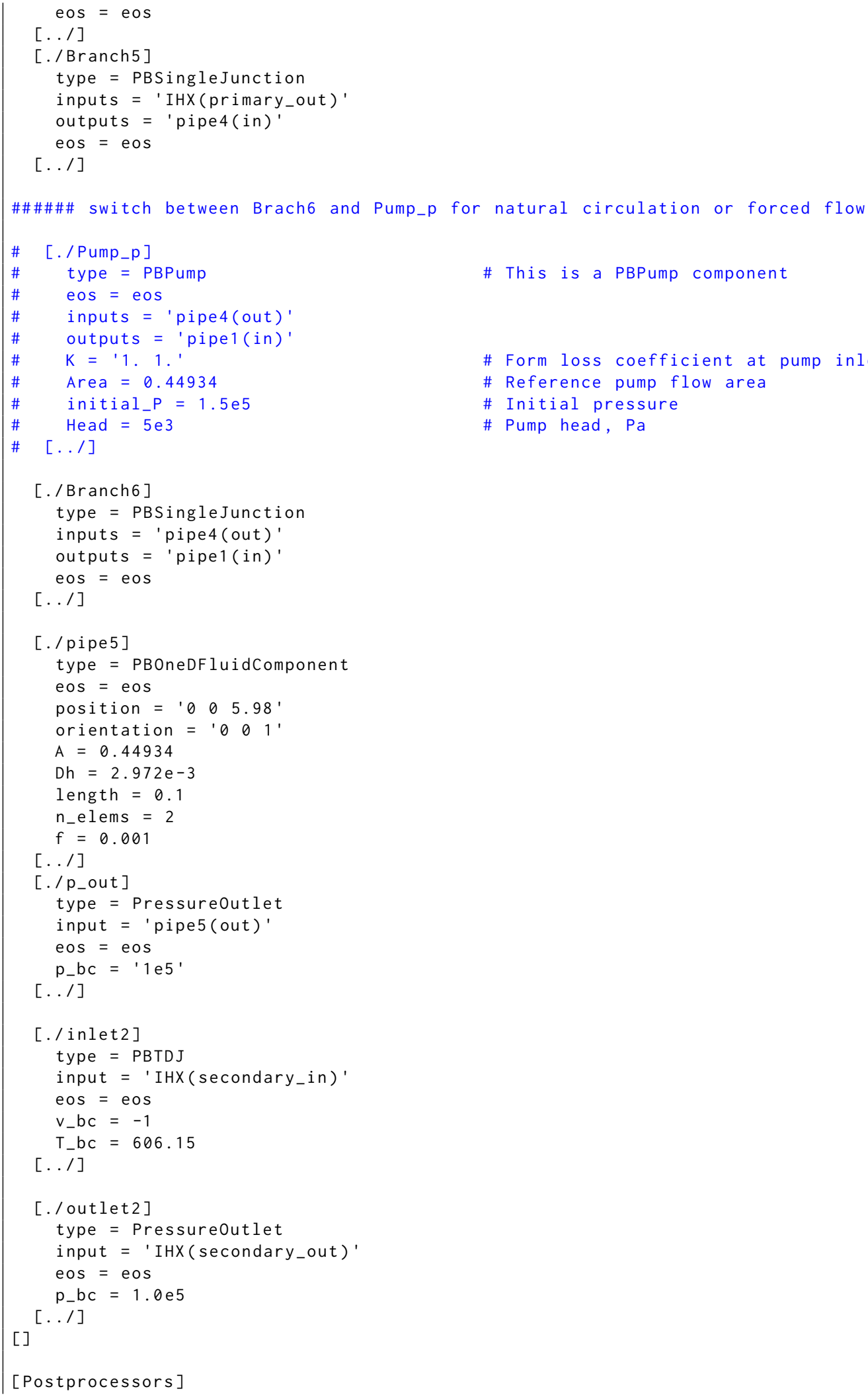




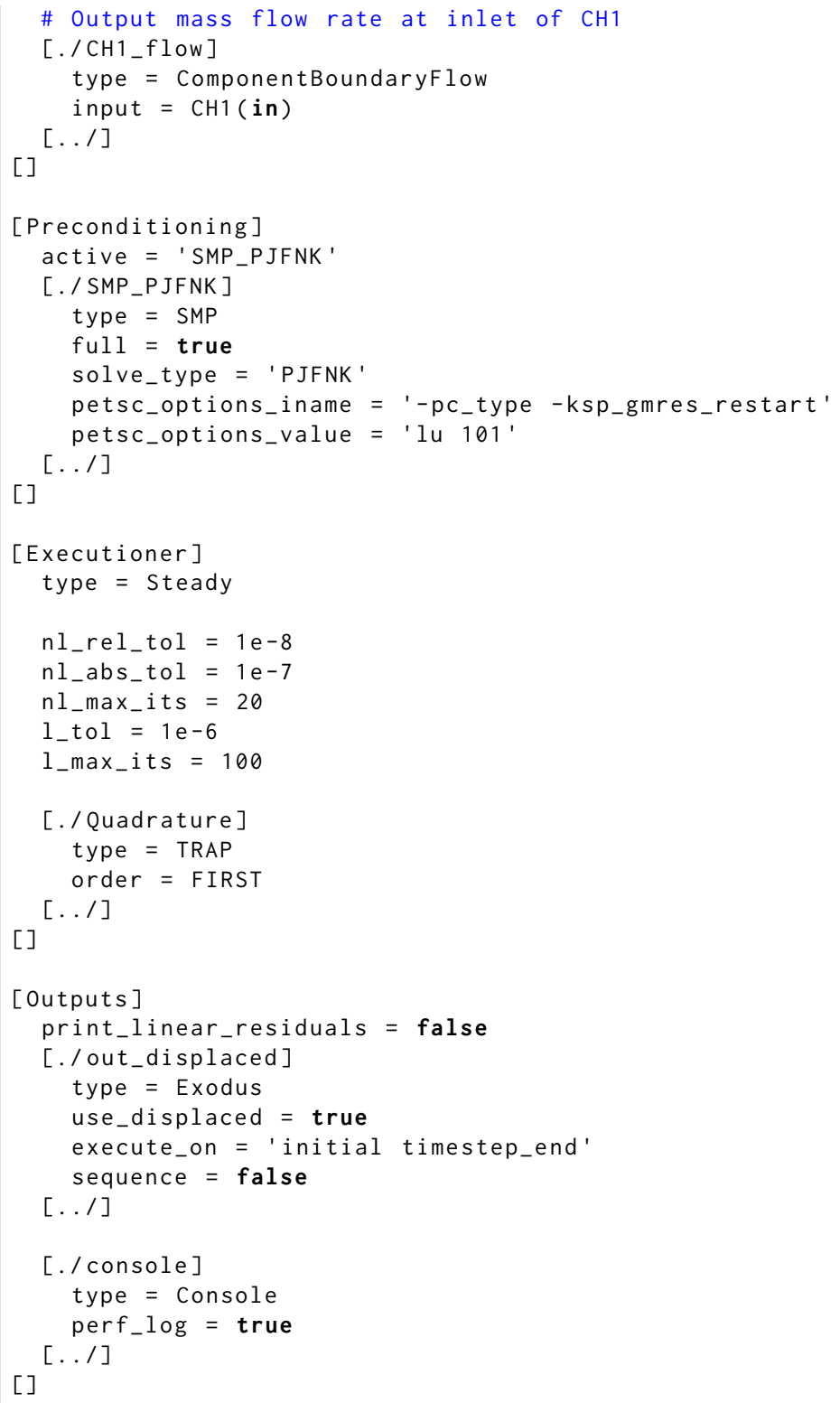




\subsection{A Simplified SFR Model}

A typical pool-type SFR test problem is presented here, based on the design information of the Advanced Burner Test Reactor (ABTR) conceptual design [17]. Figure 5.11 shows the schematics of the test SFR model. The primary coolant system consists of the downcomers (pump outlet and pump discharge), the lower plenum, the reactor core model, the upper plenum, and the intermediate heat exchanger. Five PBCoreChannels are used to describe the reactor core. PBLiquidVolume components are used to represent the cold pool and the upper plenum. Both are connected to a CoverGas component. Different components are connected with junction Components. The intermediate loop, the secondary loop, and the DRACS loop are modeled with great simplicities. Single-phase counter current heat exchanger models (PBHeatExchanger) are used to mimic the function of the intermediate loop heat exchanger (IHX), DRACS heat exchanger (DHX), and secondary loop heat exchanger (SHX) to transfer heat among the primary, intermediate, secondary, and the DRACS loops.

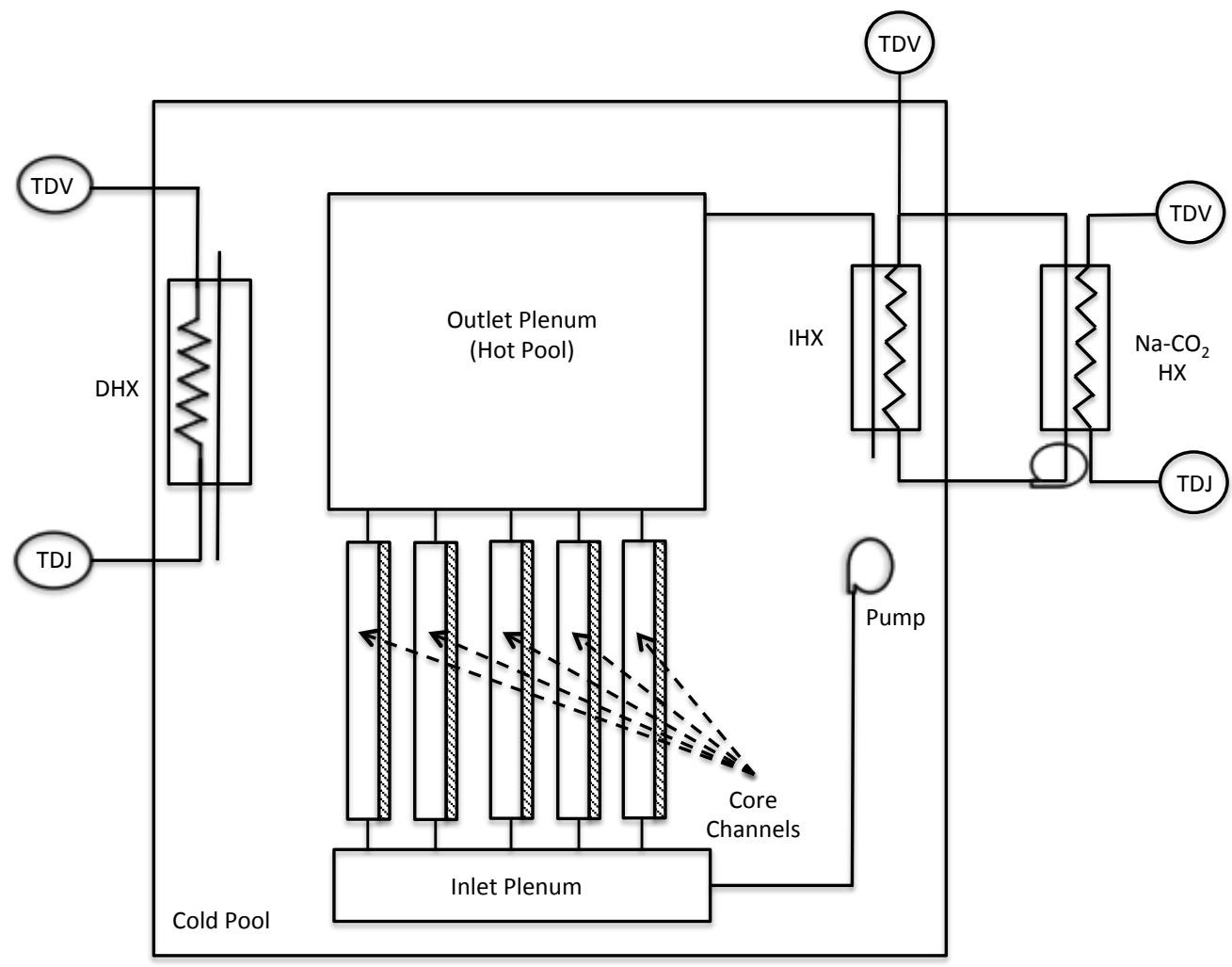

Figure 5.11: Schematics of the a simple pool-type SFR model.

The input file of this example problem is shown as follows:

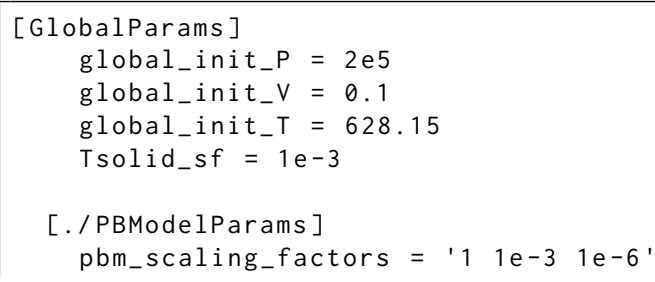




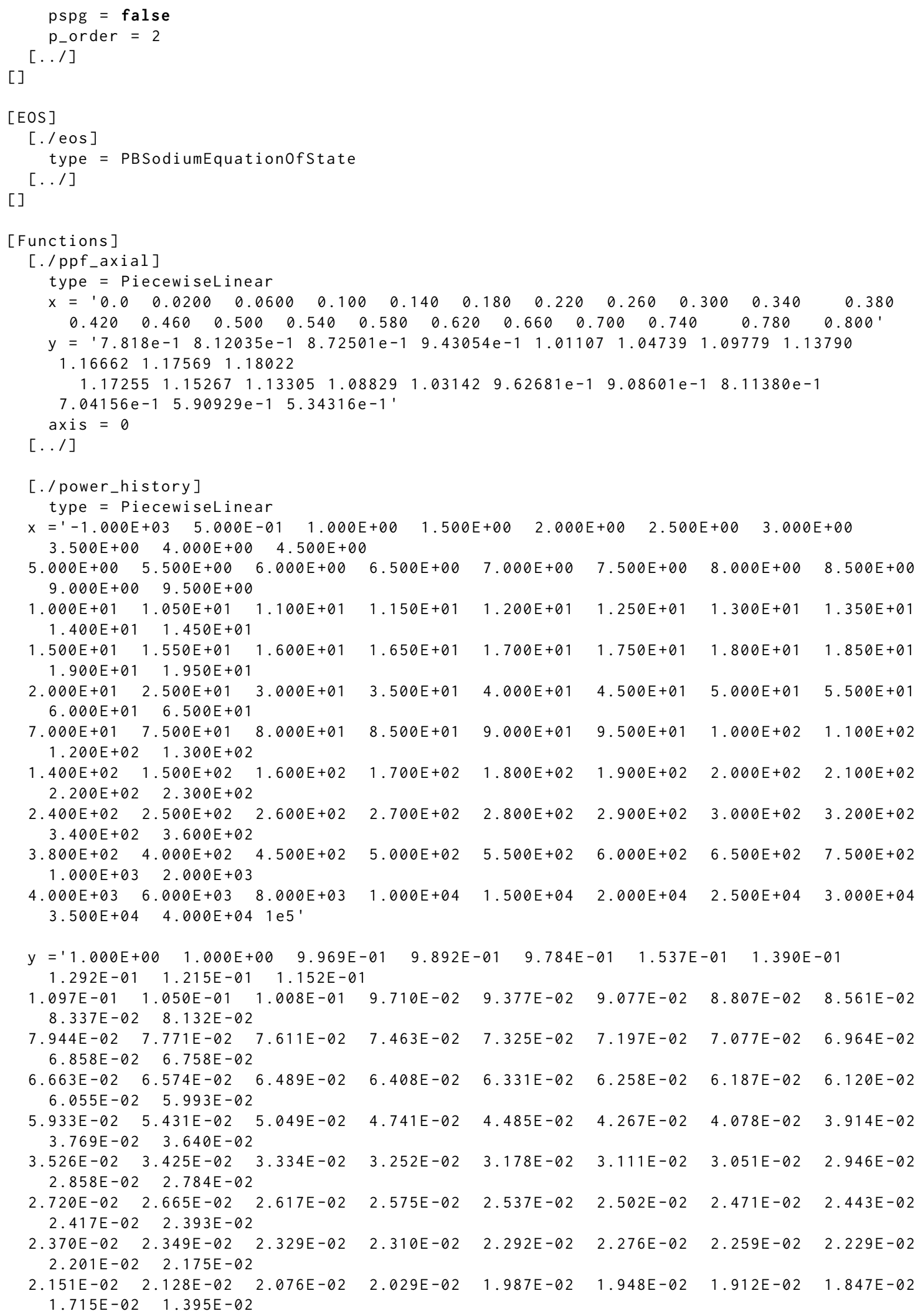




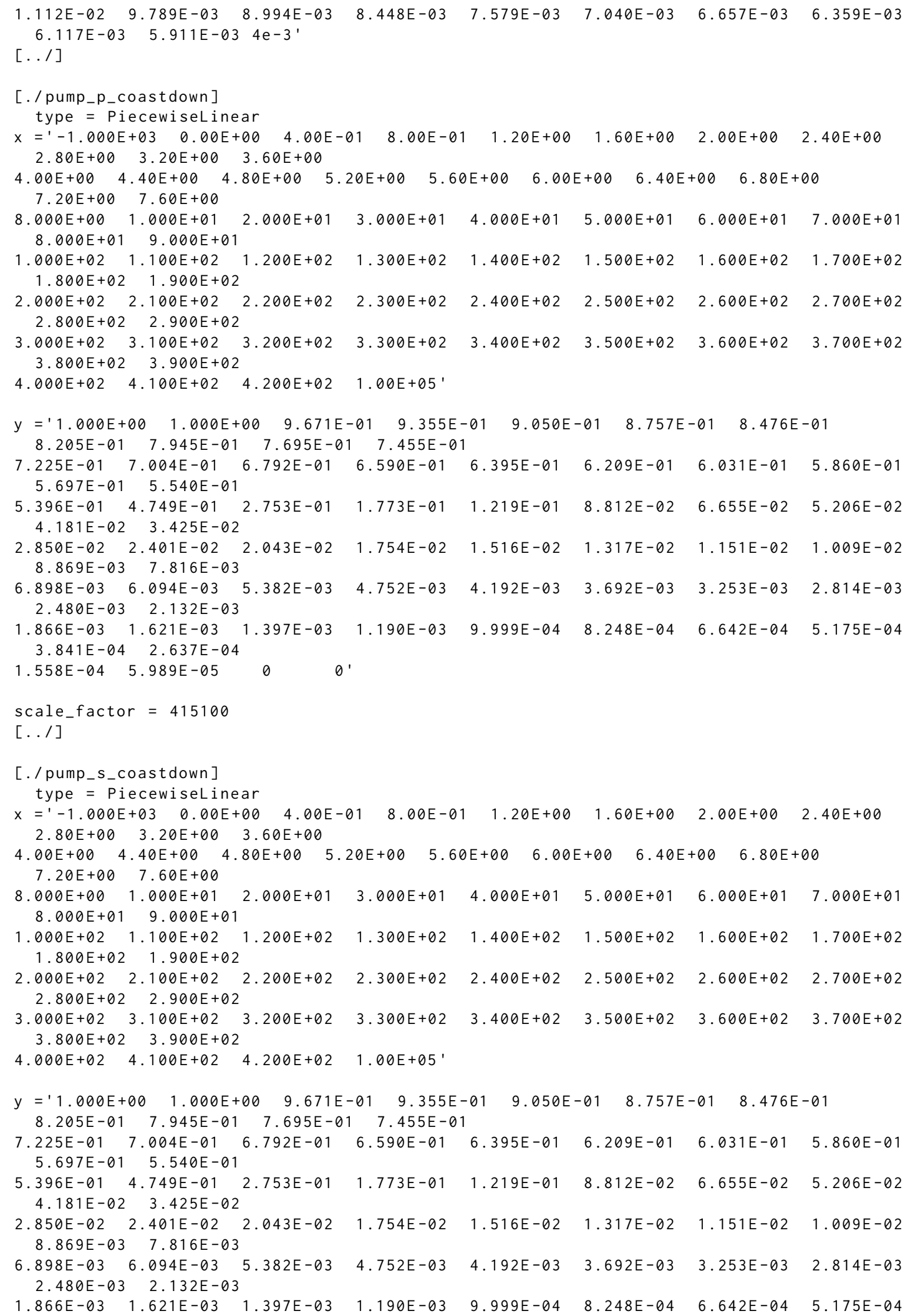




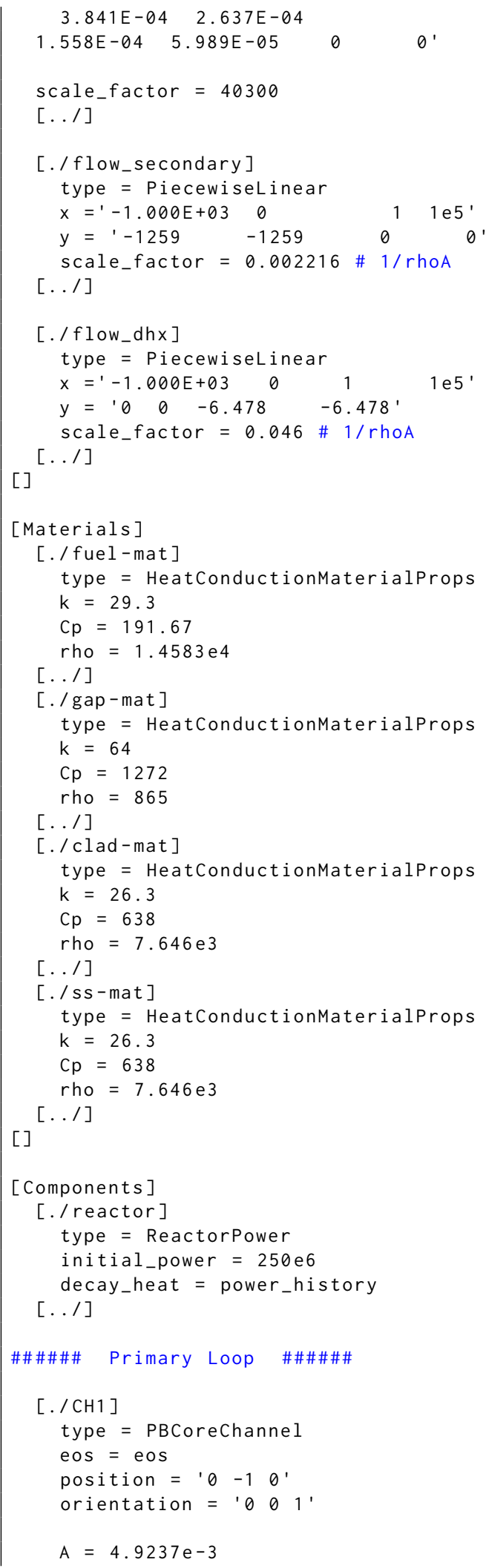




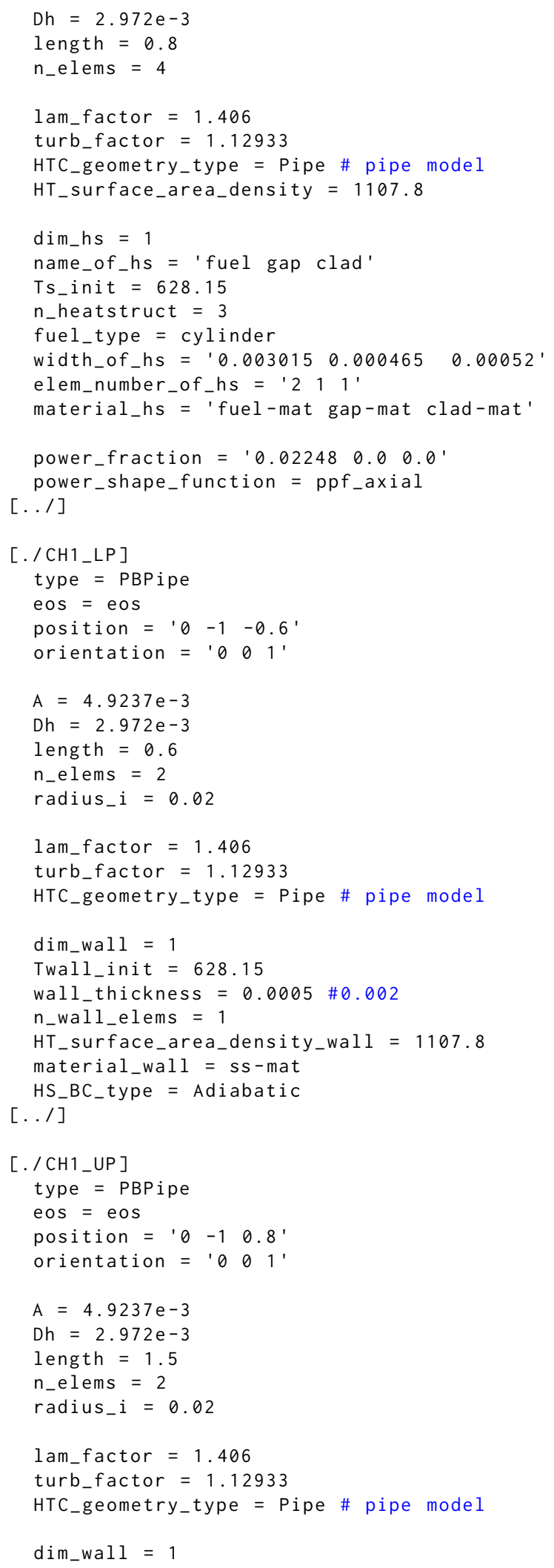




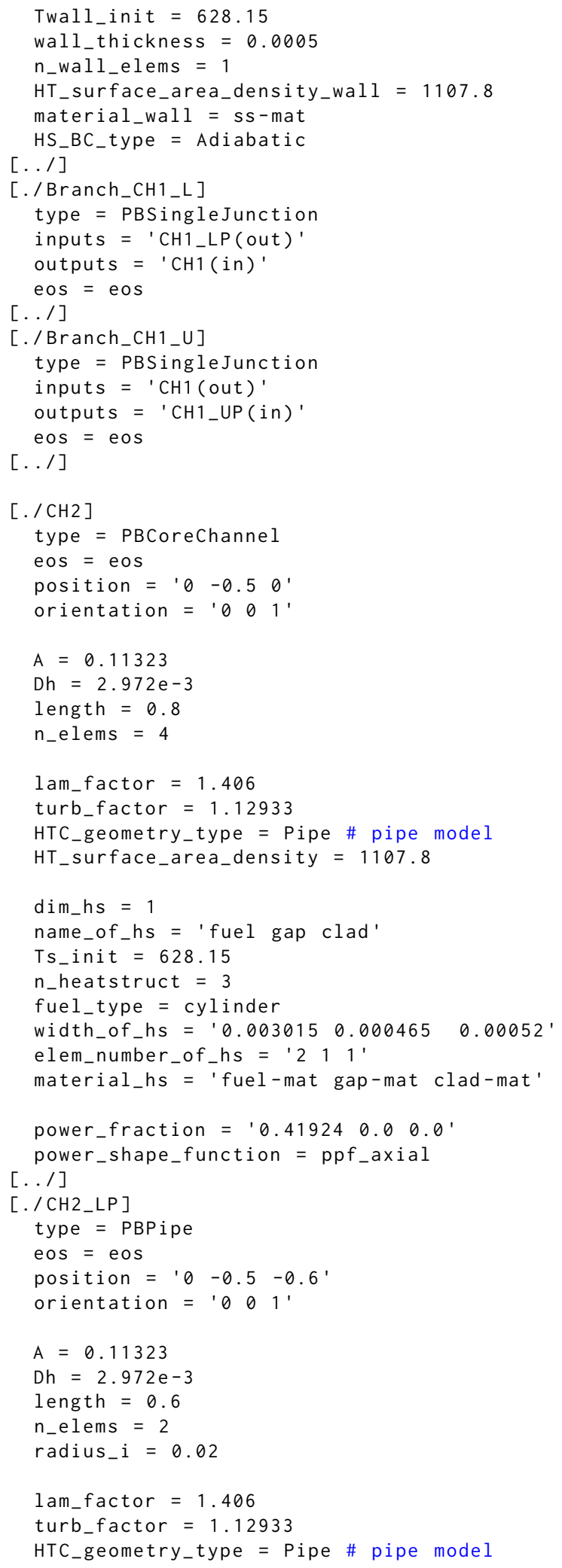




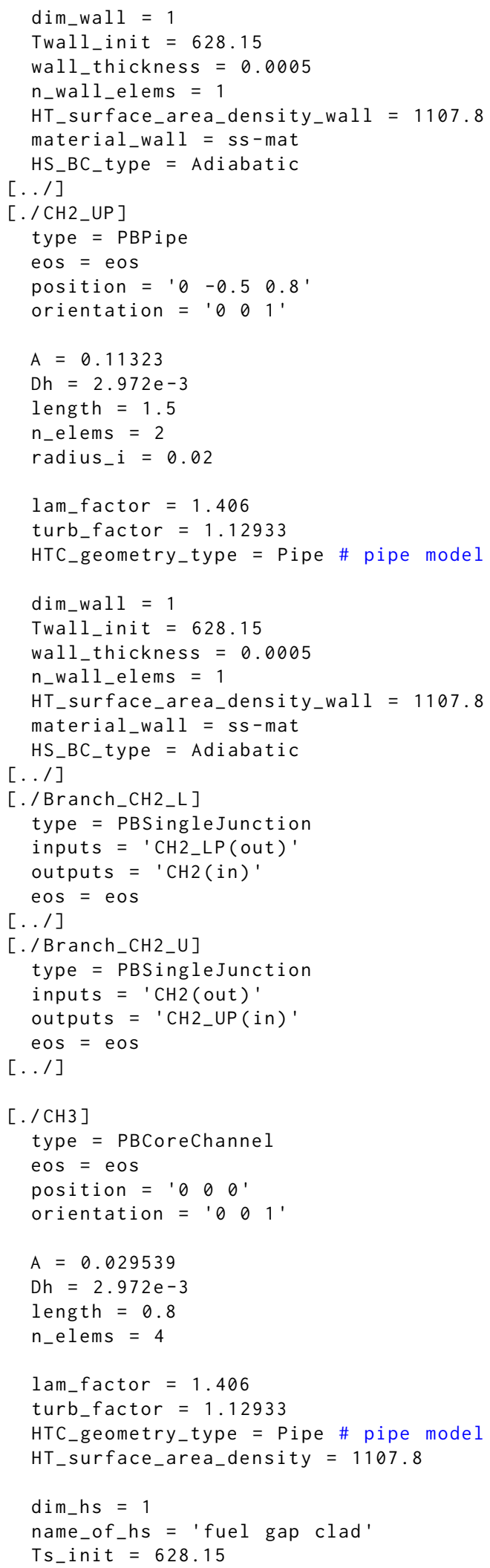




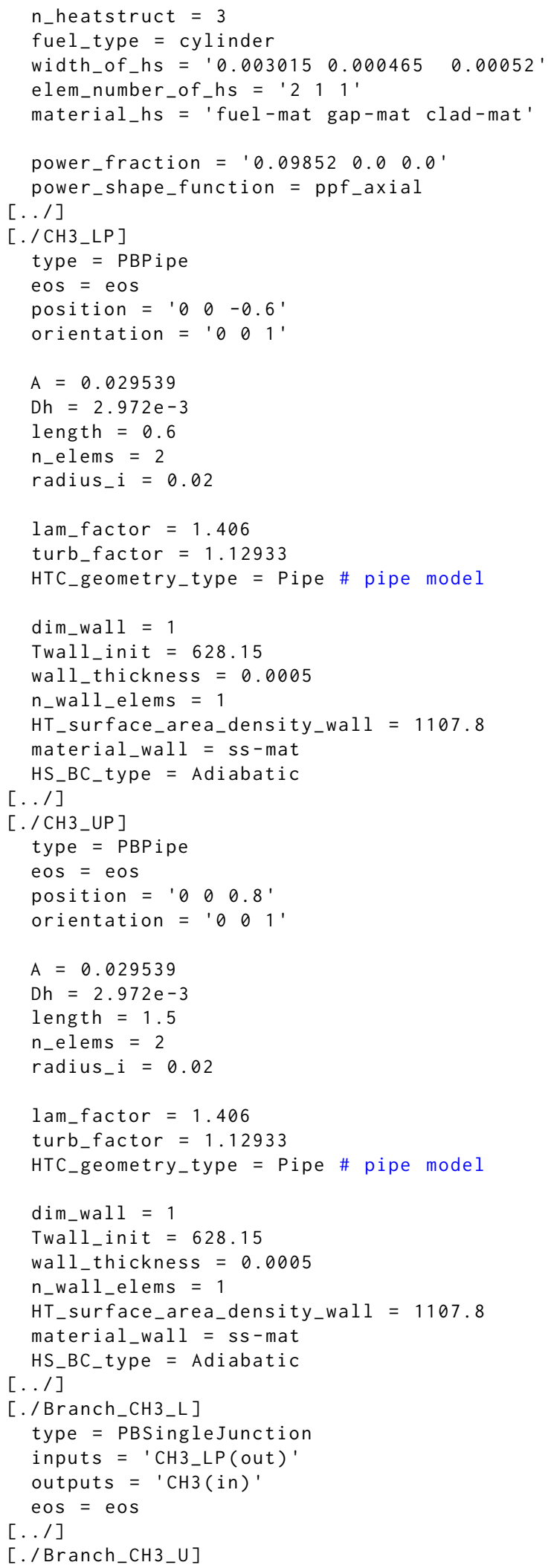




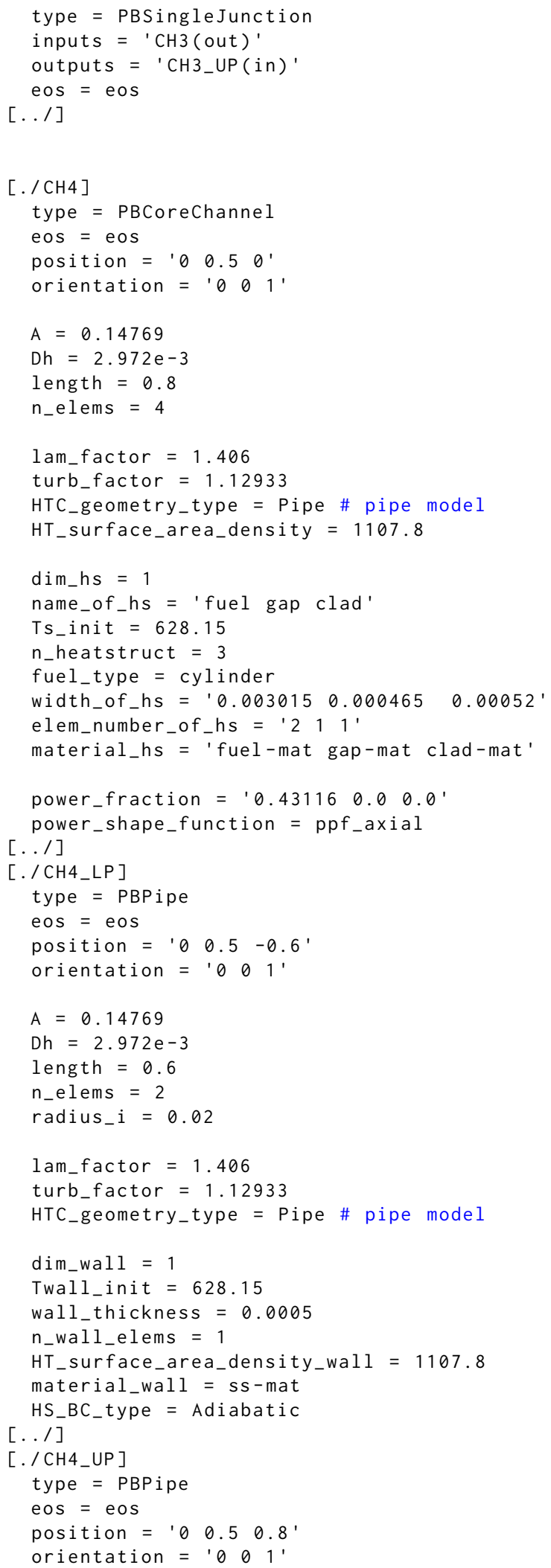




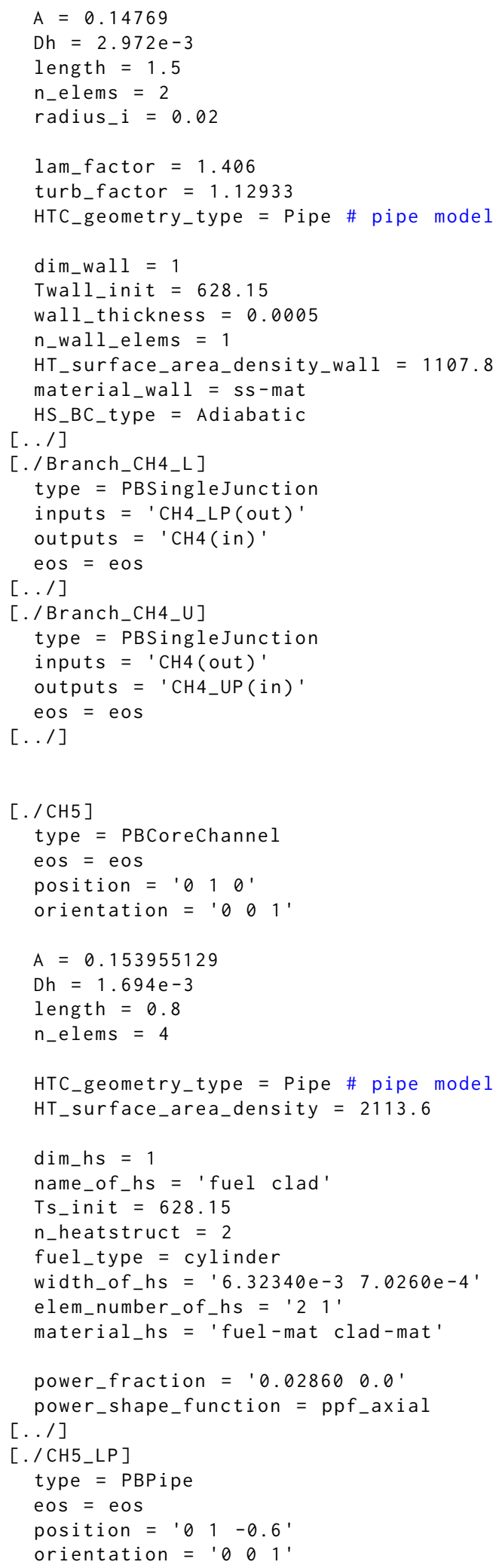




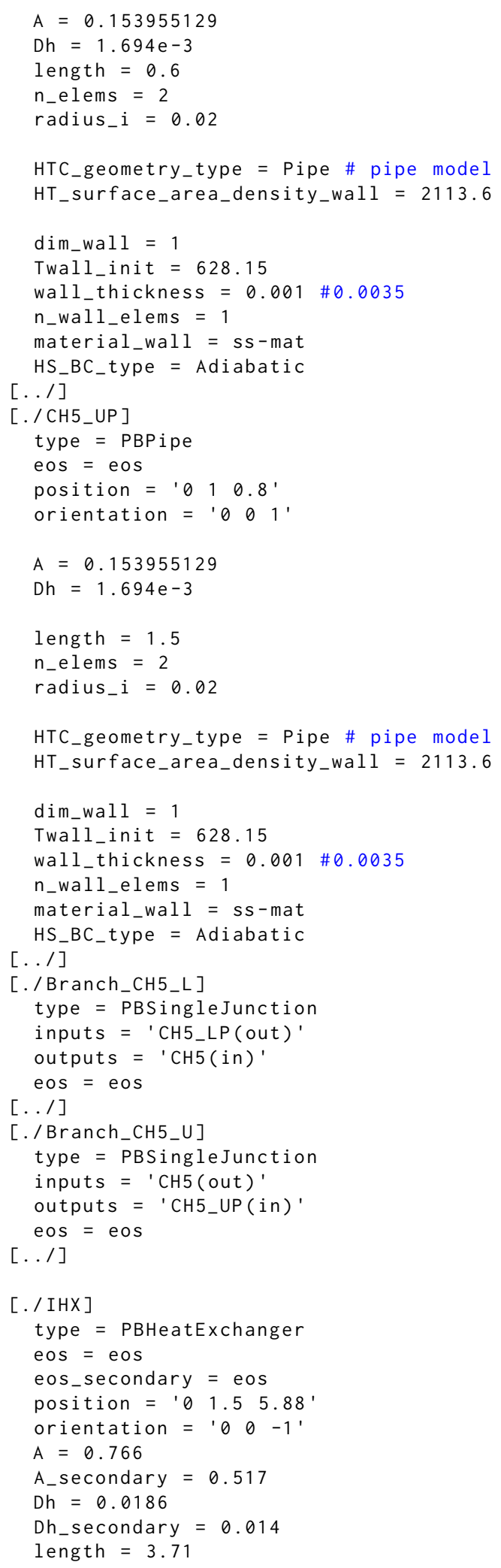




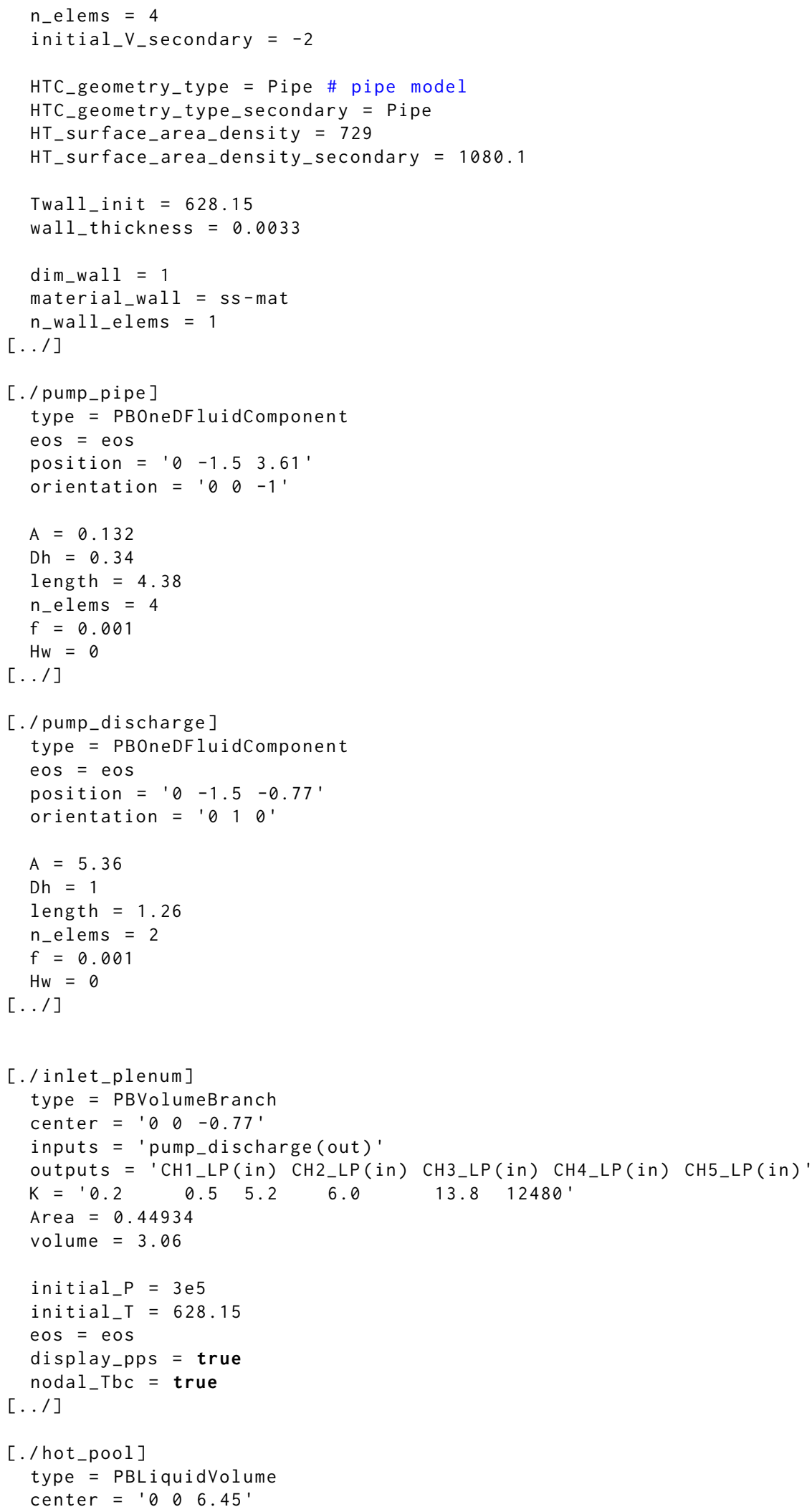




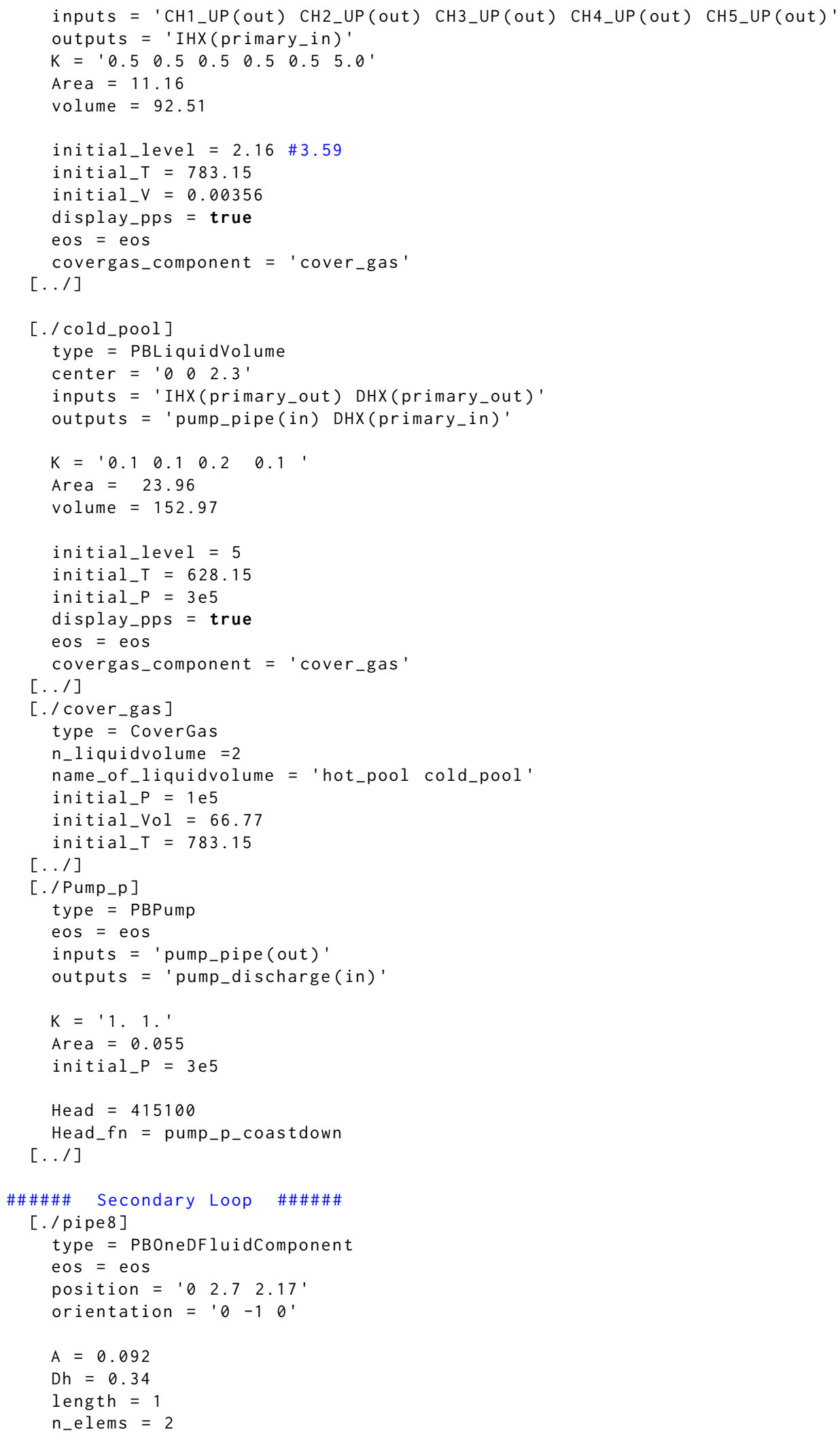




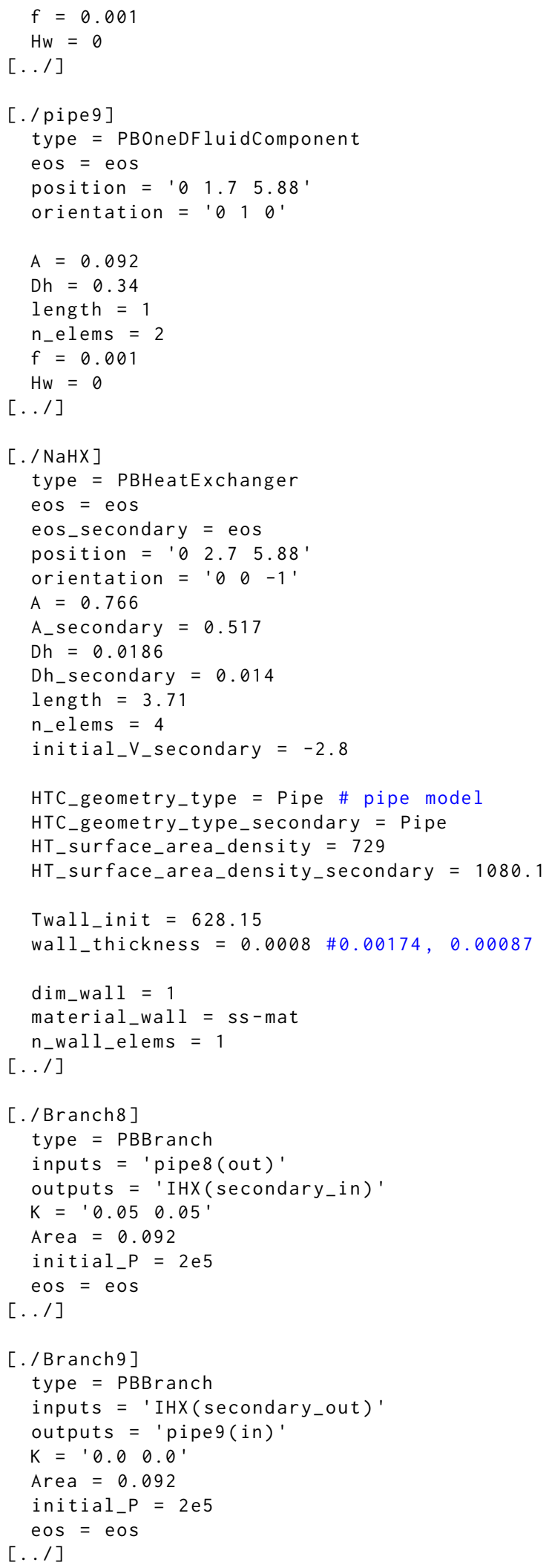




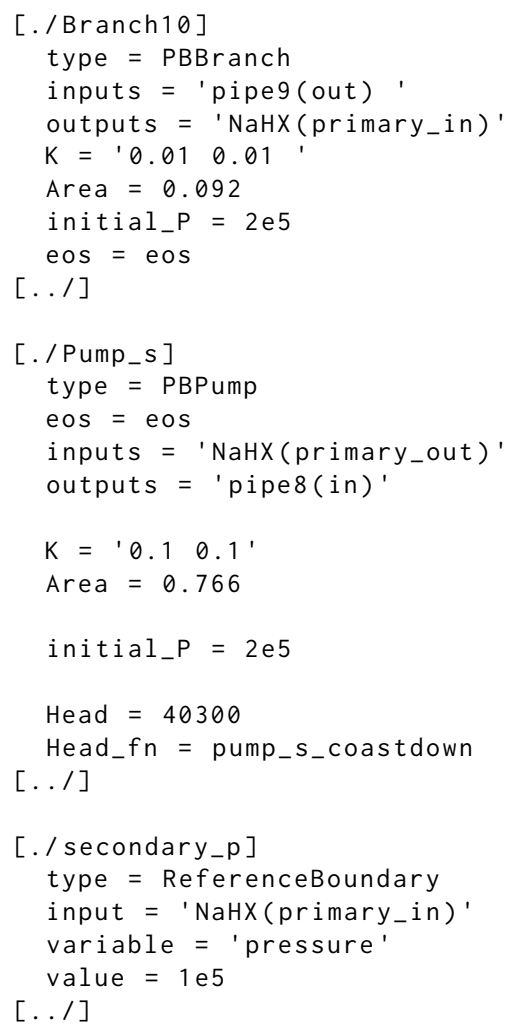




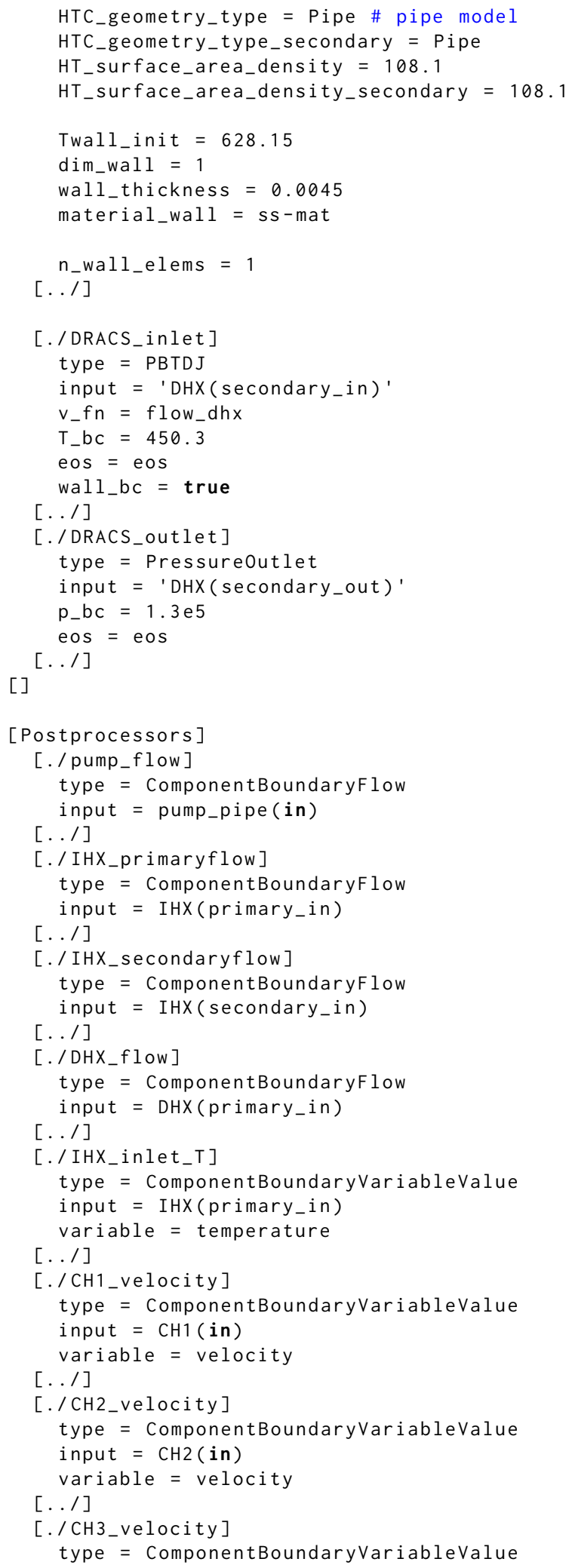




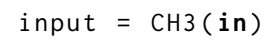

$\left[. / \mathrm{max}_{-} \mathrm{Tco} \mathrm{C}_{-} \mathrm{core}\right]$ 


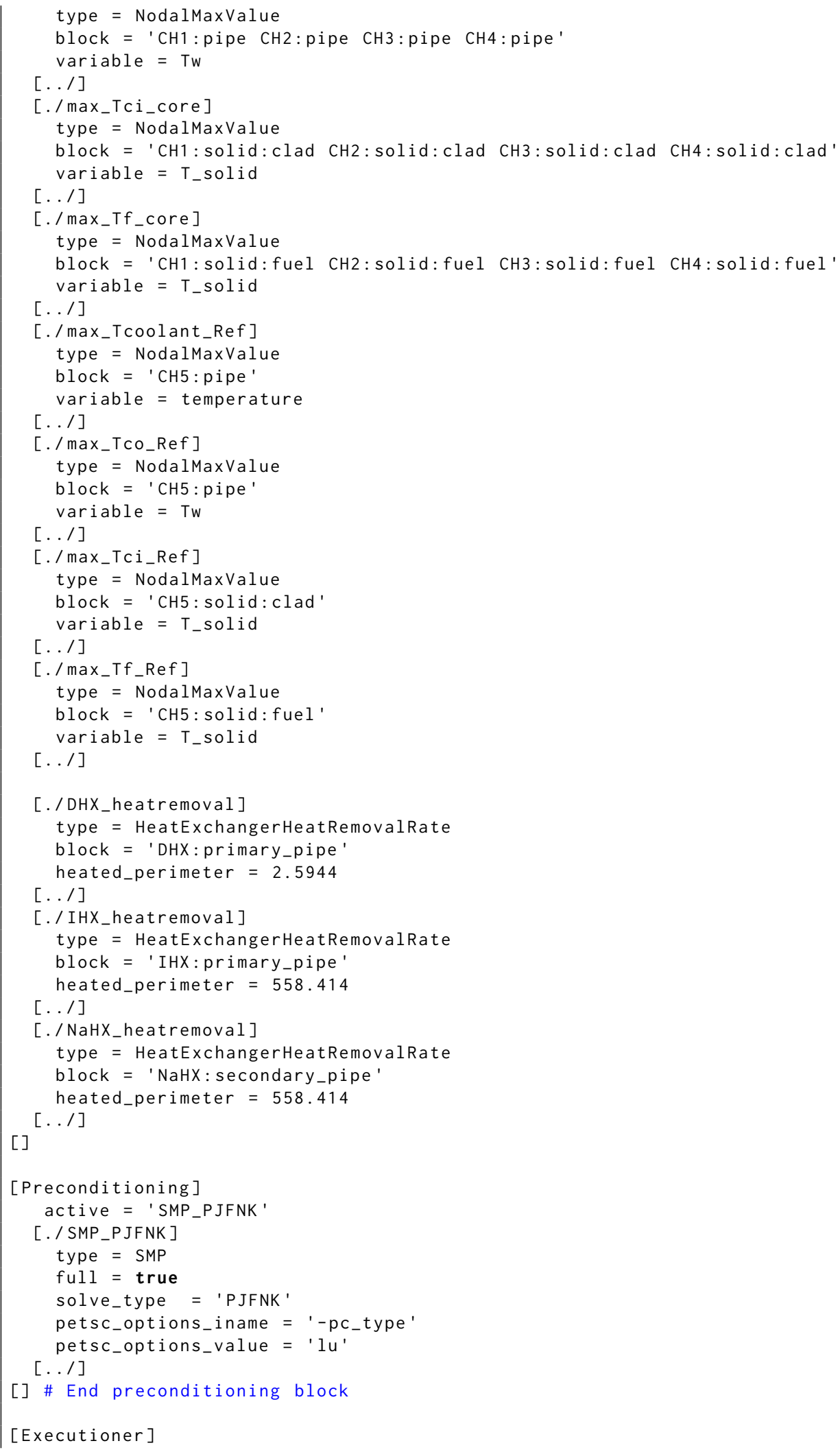




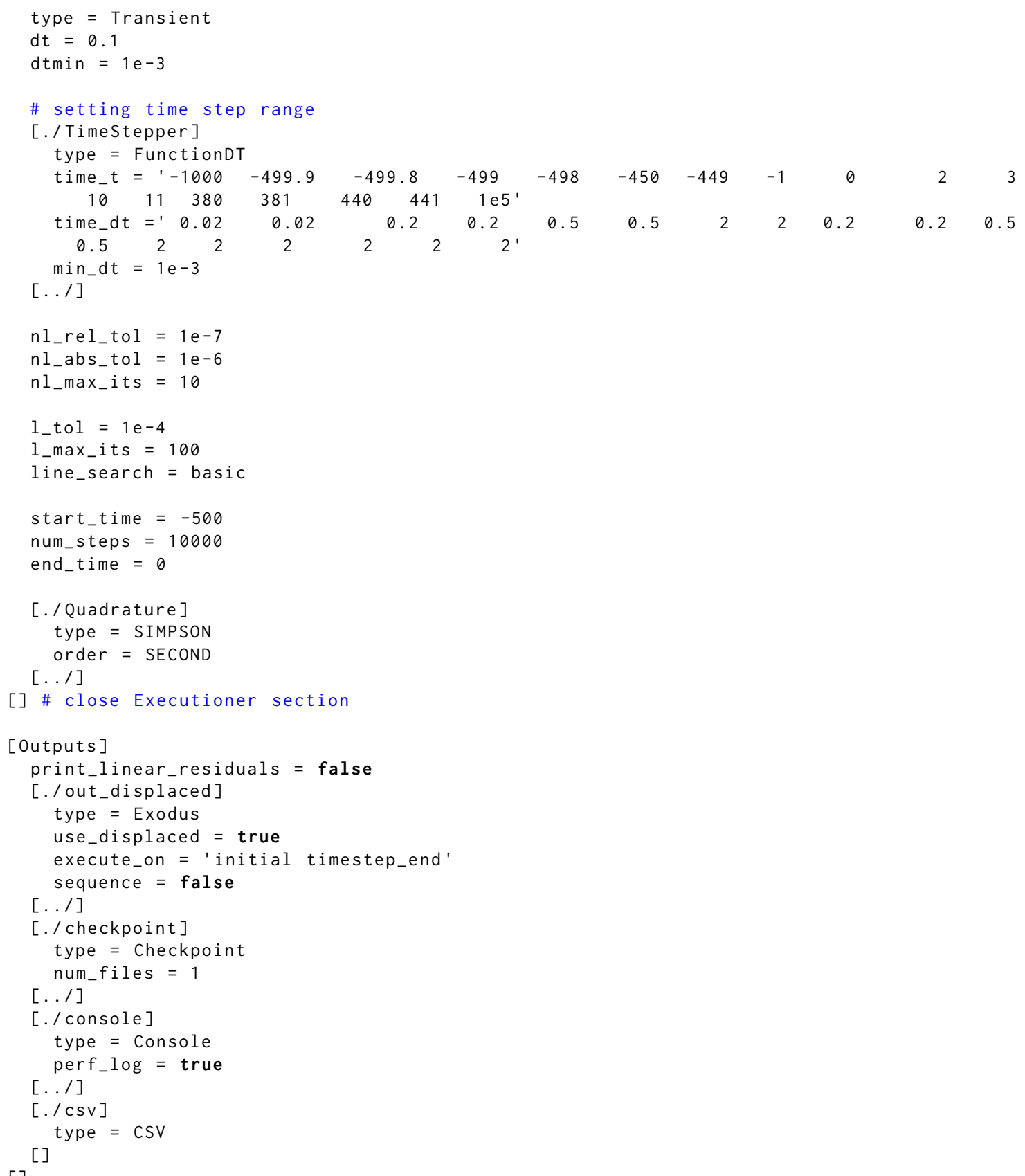




\section{ACKNOWLEDGMENTS}

This work is funded by U.S. Department of Energy Office Nuclear Energy Advanced Modeling and Simulation (NEAMS) program.

\section{REFERENCES}

[1] R. Hu. SAM Theory Manual. Technical Report ANL/NE-17/4, Argonne National Laboratory, 2017.

[2] R. Hu. Three-dimensional flow model development for thermal mixing and stratification modeling in reactor system transients analyses. Nuclear Engineering and Design, 345:209-215, 2019.

[3] G. Hu, G. Zhang, and R. Hu. Reactivity Feedback Modeling in SAM. Technical Report ANL-NSE-19/1, Argonne National Laboratory, 2019.

[4] G. Hu, R. Hu, and L. Zou. Development of Heat Pipe Reactor Modeling in SAM. Technical Report ANL-NSE-19/9, Argonne National Laboratory, 2019.

[5] D. Gaston, C. Newman, G. Hansen, and D. Lebrun-Grandié. MOOSE: A parallel computational framework for coupled systems of nonlinear equations. Nuclear Engineering and Design, 239:1768-1778, 2009.

[6] B.S. Kirk, J.W. Peterson, R.H. Stogner, and F.C. Graham. libMesh: A C++ Library for Parallel Adaptive Mesh Refinement/Coarsening Simulations. Engineering with Computers, 22:237254, 2006.

[7] PETSc. PETSc Web page., 2017. URL http://www.mcs.anl.gov/petsc.

[8] D. A. Knoll and D. E. Keyes. Jacobian-free Newton-Krylov Methods: a Survey of Approaches and Applications. Journal of Computational Physics, 193:357-397, 2004.

[9] R.A. Berry, J.W. Peterson, H. Zhang, R.C. Martineau, H. Zhao, L. Zou, D. Andrs, and J. Hansel. RELAP-7 Theory Manual. Technical Report INL/EXT-14-31366/Revision 3, Idaho National Laboratory, 2018.

[10] R. Hu, J.W. Thomas, E. Munkhzul, and T.H. Fanning. Coupled System and CFD Code Simulation of Thermal Stratification in SFR Protected Loss-Of-Flow Transients. In Proceedings of ICAPP 2014, 2014.

[11] T.H. Fanning and R. Hu. Coupling the System Analysis Module with SAS4A/SASSYS-1. Technical Report ANL/NE-16/22, Argonne National Laboratory, 2016.

[12] R. Martineau, D. Andrs, J. Hansel, C. Permann, M. Bernard, R. Johns, R. Hu, J. Wolf, H. Zhang, and R. Szilard. Extending the Capability of Nuclear Plant Systems Analysis with Advanced Tightly-Coupled Nuclear Fuels Performance. In 2018 American Nuclear Society Annual Meeting, 2018. 
[13] HYPRE Web page. URL https://computation-rnd.1lnl.gov/linear_solvers/.

[14] MPICH2 Web page. URL http://www. mcs. anl.gov/mpich2.

[15] TBB Web page. URL https://www. threadingbuildingblocks.org/.

[16] U.S. NRC. TRACE 5.0 Assessment Manual-Appendix A: Fundamental Validation Cases. Technical report, U. S. Nuclear Regulatory Commission, 2008.

[17] Y.I. Chang, P.J. Finck, and C. Grandy. Advanced Burner Test Reactor Preconceptual Design Report. Technical Report ANL-ABR-1 (ANL-AFCI-173), Argonne National Laboratory, 2006. 


\section{Argonne}

Nuclear Science \& Engineering Division

Argonne National Laboratory

9700 South Cass Avenue, Bldg. 208

Argonne, IL 60439

www.anl.gov 